\title{
Metallic Glass Matrix Composites
}

\author{
Junwei Qiao1 ${ }^{, 3, *}$, Haoling Jia ${ }^{2}$, and Peter K. Liaw ${ }^{2, * *}$ \\ ${ }^{1}$ Key Laboratory of Interface Science and Engineering in Advanced Materials, Ministry \\ of Education, Taiyuan University of Technology, Taiyuan 030024, China \\ ${ }^{2}$ Department of Materials Science and Engineering, The University of Tennessee, \\ Knoxville, TN 37996-2200, USA \\ ${ }^{3}$ Laboratory of Applied Physics and Mechanics of Advanced Materials, College of \\ Materials Science and Engineering, Taiyuan University of Technology, Taiyuan \\ 030024, China
}

\begin{abstract}
The mechanical properties of ex-situ and in-situ metallic glass matrix composites (MGMCs) have proven to be both scientifically unique and of potentially important for practical applications. However, the underlying deformation mechanisms remain to be studied. In this article, we review the development, fabrication, microstructures, and properties of MGMCs, including the room-temperature, cryogenic-temperature, and high-temperature mechanical properties upon quasi-static and dynamic loadings. In parallel, the deformation mechanisms are experimentally and theoretically explored. Moreover, the fatigue, corrosion, and wear behaviors of MGMCs are discussed. Finally, the potential applications and important unresolved issues are identified and discussed.
\end{abstract}

\footnotetext{
* Corresponding authors. Tel.: 863516018051

*E-mail address: qiaojunwei@gmail.com (J.W. Qiao)

${ }^{* *}$ E-mail address: pliaw@utk.edu (P.K. Liaw)
} 


\section{Introduction}

\subsection{Development of bulk metallic glasses}

Before 1960, highly-disordered arrangements of atoms, similar to that of a liquid state, have never been found in solid metals and alloys. In 1960, the first reported metallic glass, scientifically obtained, was the alloy, $\mathrm{Au}_{75} \mathrm{Si}_{25}$ (It is noted that all of the compositions in the text are in atomic percent, at. \%) produced at the California Institute of Technology (Caltech) of USA by Klement, Willens, and Duwez [1]. The so-called metallic glass is the one that retains the disordered atomic structure of high-temperature melts. Figure 1(a) shows the amorphous structure of the equimolar CuZr alloys with 200 atoms by computer simulations. It is impossible to locate the atoms by translation and rotation operations in the disordered structure. In contrast, atoms in the equimolar cubic crystalline $\mathrm{CuZr}$ phase have a regular arrangement, as shown in Figure 1(b). The early glass-forming alloys had to be cooled extremely rapidly to avoid crystallization. Usually, the metallic glasses could only be produced into ribbons, films, or wires, since heat could be extracted quickly enough to achieve the critical cooling rate of $10^{5}-10^{6} \mathrm{~K} / \mathrm{s}$. In the early $1970 \mathrm{~s}$, low-dimensional metallic glasses, such as amorphous ribbons and wires, mainly included magnetic Fe- and Co-based ribbons, which were focused on their excellent softer magnetic properties [2]. Meanwhile, based on Turnbull's criterion: a liquid with $T_{\mathrm{g}} / T_{\mathrm{m}}=2 / 3\left(T_{\mathrm{g}}\right.$ and $T_{\mathrm{m}}$ denoting the glass-transition and melting temperatures, respectively) becomes very sluggish in crystallization, and a glass alloy is easily to be obtained, researchers were 
trying their best to find "bulk" metallic glasses. One arbitrarily defines the millimeter scale as "bulk".

In 1974, Chen firstly found bulk metallic glasses (BMGs) in the ternary $\mathrm{Pd}-\mathrm{Cu}-\mathrm{Si}$ alloy, which yielded a cylindrical sample, 1 - $3 \mathrm{~mm}$, in diameter and several cm in length with a critical cooling rate to be less than $10^{3} \mathrm{~K} / \mathrm{s}$ [3]. In 1982, a bulk glassy ingot could be produced with a composition of $\mathrm{Pd}_{40} \mathrm{Ni}_{40} \mathrm{P}_{20}$, and it was slowly cooled $(1.4 \mathrm{~K} / \mathrm{s})$ on a fused silica surface [4]. In 1980s, more and more BMGs were developed in various alloy systems, and there were considerable efforts on the fundamental understanding of structural, atomic, and electronic transport properties and low-temperature behavior as well as further exploration of mechanical, magnetic, and chemical properties [5].

During the late 1980s, Inoue's group at Tohoku University of Japan succeeded in developing many multicomponent BMG systems, consisting mainly of common metallic elements with lower critical cooling rates. For example, they found the exceptional glass-forming ability in $\mathrm{La}-\mathrm{Al}-\mathrm{Ni}$ and $\mathrm{La}-\mathrm{Al}-\mathrm{Cu}$ alloys [6]. Cylindrical samples with diameters of up to $5 \mathrm{~mm}$ or sheets with similar thicknesses were made fully glassy by casting $\mathrm{La}_{55} \mathrm{Al}_{25} \mathrm{Ni}_{20}$. By high-pressure die casting, the $\mathrm{Mg}_{80} \mathrm{Cu}_{10} \mathrm{Y}_{10} \mathrm{BMGs}$ with a maximum thickness of about $10 \mathrm{~mm}$ in cylindrical and sheet shapes could be produced [7]. Moreover, in the early 1990s, the $\mathrm{Zr}_{65} \mathrm{Al}_{7.5} \mathrm{Ni}_{10} \mathrm{Cu}_{17.5}$ BMGs were fabricated by water quenching with a diameter up to 16 $\mathrm{mm}$ [8], and the $\mathrm{Zr}_{55} \mathrm{Al}_{10} \mathrm{Ni}_{5} \mathrm{Cu}_{30}$ BMGs were produced by suction-casting the molten alloys into a copper mold with a diameter of $16 \mathrm{~mm}$ [9]. In 1997, the $\mathrm{Pd}-\mathrm{Cu}-\mathrm{Ni}-\mathrm{P}$ 
family with the super high glass-forming ability (GFA) and with a critical casting diameter of $72 \mathrm{~mm}$ was discovered in Inoue's group [10]. It is one of the significant breakthroughs during the development of BMGs. Another significant breakthrough is the development of a $\mathrm{Zr}_{41.2} \mathrm{Ti}_{13.8} \mathrm{Cu}_{12.5} \mathrm{Ni}_{10} \mathrm{Be}_{22.5} \mathrm{BMG}$, commonly referred to as Vitreloy 1 (Vit1), with a critical cooling rate less than $10 \mathrm{~K} / \mathrm{s}$ in Johnson's group from Caltech [11]. Up to now, the world's largest glassy alloy ever made is $\mathrm{Pd}_{42.5} \mathrm{Cu}_{30} \mathrm{Ni}_{7.5} \mathrm{P}_{20}$, which can be cast into amorphous rods in a 80 -mm diameter by copper-mould casting in 2012 [12]. Table 1 exhibits some of BMGs with the critical diameters larger than $10 \mathrm{~mm}$ by various methods [8, 10-34]. Figure 2 shows some maximum diameters of the BMG systems with their discovered years.

With the coming of the 21 century, the widespread enthusiasm for research on BMGs is driven by both a fundamental interest in the structures and properties of disordered materials and their unique promise for structural and functional applications. Specially, BMGs are considered as potential candidates in the structural engineering field, since the most enduring attractions of BMGs are their impressive suite of mechanical properties [35]. It is well known that BMGs exhibit high strengths (even an ultrahigh strength of over $5 \mathrm{GPa}$ [35]), high hardness, high specific strengths (strength / density), superior elastic limits (2\%), high scratch and wear resistances, etc. However, the lack of the macroscopic plasticity or ductility severely limits their structural applications at room temperature. In the late 1990s, some research shifted towards toughening strategies for BMGs by developing in-situ and ex-situ metallic glass matrix composites (MGMCs), which possess high toughness and inhibit the rapid 
propagation of shear bands. As a result, combining the high strength and toughness makes MGMCs promising as structural engineering applications.

In this article, we review recent advances in in-situ and ex-situ MGMCs, with particular emphases on the deformation and fracture mechanisms. Atomistic as well as continuum modeling and experimental work on deformation behavior under various loadings are discussed, and theoretical developments are described.

\section{$1.2 \quad$ Ex-situ bulk MGMCs}

In searching for bulk shapes of amorphous alloys, i.e., BMGs, Inoue's group from Tohoku University contributed substantially to the progress, as discussed above. In parallel, in designing toughening BMGs for mechanical applications, Johnson's group from Caltech made very great contributions, and a series of in-situ and ex-situ MGMCs have been successfully found (discussed below).

Monolithic BMGs usually fail with the localized deformation at room temperature, caused by the prompt propagation of single initiated shear bands [35]. The so-called shear bands in BMGs are the deformation bands with a thickness of only 10 $\mathrm{nm}[36]$, distributed on the lateral surface of deformed samples, much less than the thickness of deformation bands in conventional crystalline alloys with a typical value of several microns. Plastic deformation of metallic glasses is entirely localized into thin shear bands. In order to avoid the early failure of monolithic BMGs upon loading, the simplest method that could be easily considered is to add secondary phases, which can directly arrest the evolution of shear bands into macro-cracks and encourage the formation of multiple shear bands. This kind of dual-phase composites is ex-situ 
MGMCs. The category of such ex-situ composites includes the particle-reinforced and fiber-reinforced MGMCs, according to the continuity of secondary phases, i.e., by reinforcing alloys with particles, long or short fibers.

\subsubsection{Particle-reinforced MGMCs}

The first ex-situ MGMCs (particle reinforced) were synthesized by Choi-Yim and Johnson in 1997 [37]. The nominal compositions of the three alloys:

$\mathrm{Cu}_{47} \mathrm{Ti}_{34} \mathrm{Zr}_{11} \mathrm{Ni}_{8}, \mathrm{Zr}_{52.5} \mathrm{Ti}_{5} \mathrm{Al}_{10} \mathrm{Cu}_{17.9} \mathrm{Ni}_{14.6}(\mathrm{~V} 105)$, and $\mathrm{Zr}_{57} \mathrm{Nb}_{5} \mathrm{Al}_{10} \mathrm{Cu}_{15.4} \mathrm{Ni}_{12.6}(\mathrm{~V} 106)$ were selected as the glass matrices. Ceramics, such as $\mathrm{SiC}$, WC, or TiC, and the metals, $\mathrm{W}$ or Ta, were used as secondary phases. The volume fractions (vol. \%) of particles ranged from $5 \%-30 \%$, and the sizes of the particles varied between 20 and $80 \mu \mathrm{m}$. Short [aspect ratio (length / diameter) $=3$ ] tungsten wires with a diameter of $100 \mu \mathrm{m}$ were also used with a volume fraction of $10 \%$. A mixture of the pre-alloyed metallic glass-forming elements and second phases were combined by induction melting. The composite ingots were, then, remelted at temperatures ranging from 850 to $1,373 \mathrm{~K}$ under vacuum in a quartz tube using an induction-heating coil and, then, injected through a nozzle into a copper mold using the high-purity argon under a 1-atm pressure.

Figure 3(a) shows an optical micrograph of the uniformly-distributed WC particles in the V106 matrix, which is the first report on the synthesis of ex-situ MGMCs [37]. The size of particles is about $50 \mu \mathrm{m}$, and the vol. \% of WC is $10 \%$ [37]

. Figure 3(b) presents the X-ray diffraction patterns of the V106 matrix, the alloy reinforced with 10 vol. \% WC, and pure WC particle [38]. The X-ray results of the composite present diffraction peaks from WC particles superimposed on the broad 
diffuse scattering maxima from the metallic glass matrix, and no other crystalline phases can be detected. The optical micrograph together with the X-ray diffraction indicates the successful fabrication of particle-reinforced MGMCs with uniform dispersions.

Figure 3(c) shows the quasi-static compression stress-strain curves of V106 composites reinforced with Ta, W, and WC particles [39]. The unreinforced V106 had only the plastic deformation of $\sim 0.5 \%$ in compression [39]. The plastic strain of the tungsten-reinforced samples was $\sim 7 \%$, whereas the samples reinforced with Ta and WC showed a less plastic strain $(\sim 3-5 \%)$ from Figure 3(c). The compression samples made from $\mathrm{SiC}$ particles and 3- $\mu \mathrm{m} \mathrm{W}$ particles had no plasticity [39].

Up to now, there exist many different particle-reinforced ex-situ MGMCs, such as $\mathrm{WC}_{\mathrm{p}} / \mathrm{Zr}$-based [37-39], $\mathrm{WC}_{\mathrm{p}} / \mathrm{Cu}$-based [37], $\mathrm{SiC}_{\mathrm{p}} / \mathrm{Zr}$-based [37-39], $\mathrm{SiC}_{\mathrm{p}} /$ Cu-based [37, 38], Tap / Zr-based [37-40], TiB $2 \mathrm{p} / \mathrm{Mg}$-based [41], $\mathrm{ZrC}_{\mathrm{p}} / \mathrm{Zr}$-based [42], porous W / Zr-based [43, 44], porous Mo / Mg-based [45, 46], porous Ti / Mg-based [47], $\mathrm{Ti}_{\mathrm{p}} / \mathrm{Mg}$-based [48], porous $\mathrm{SiC} / \mathrm{Zr}$-based [49], $\mathrm{Cu}_{\mathrm{p}} / \mathrm{Cu}$-based [50], $\mathrm{TiNb}_{\mathrm{p}}$ /Zr-based [51], $\mathrm{Ti}_{\mathrm{p}} /$ La-based [52], and $\mathrm{C}_{\mathrm{p}} /$ Zr-based bulk MGMCs (the subscript, p, denoting particles) [53]. All these ex-situ dual-phase composites exhibit toughness at room temperature. Figure 4 presents the scanning-electron-microscopy (SEM) image of the transverse cross section of the $\mathrm{Mg}_{65} \mathrm{Cu}_{25} \mathrm{Gd}_{10}$ MGMC with 40 vol. \% Ti powders [48]. It is found that the Ti powders maintain its original size and shape with a diameter less than about $150 \mu \mathrm{m}$, and disperse very homogeneously in the glassy matrix. This trend indicates that Ti powders did not dissolve into the Mg-based glassy matrix, 
demonstrating that the Mg-based molten alloys have good castability and high fluidity as well as good wetability with Ti powders. Conventional Mg alloys with the high specific strength are widely used in structural applications. Here, current 40 vol. $\% \mathrm{Ti}_{\mathrm{p}} /$ Mg-based MGMCs have a higher specific strength of about $3.3 \times 10^{5} \mathrm{Nm} / \mathrm{kg}$ with the remarkable plastic deformation of $40 \%$ [48].

By adding the ductile metals, such as Ti particles, or brittle intermetallics, such as $\mathrm{ZrC}$ particles, into the glass matrix, the intrinsic brittleness of monolithic BMGs is greatly improved. Meanwhile, the other mechanical properties, such as hardness, are enhanced. For instance, due to the increase of $\mathrm{ZrC}$ in the $\mathrm{Zr}_{55} \mathrm{Al}_{10} \mathrm{Ni}_{5} \mathrm{Cu}_{30}$ glass matrix, the hardness of the composites is obviously increased, as shown in Figure 5(a) [42], and the yielding strength and elastic modulus exhibit a close-to-linear increase with the increase in the $\mathrm{ZrC}$ content, ranging from 0 to 20 vol. \%, as exhibited in Figure 5(b) [42]. A similar phenomenon has been found in $\mathrm{TiB}_{2 \mathrm{p}} / \mathrm{Mg}$-based MGMCs with the highest specific strength of $3.5 \times 10^{5} \mathrm{Nm} / \mathrm{kg}$ among all the existing alloys, including all commercial light-weight alloys based on $\mathrm{Mg}, \mathrm{Al}$, and $\mathrm{Ti}$, as well as high-strength steels, and Zr-based BMGs [41].

It should be noted that some MGMCs include a very high volume fraction of secondary phases, and someone argues that whether such kinds of dual-phase composites should have a metallic glass matrix or crystalline matrix. Here, we simply define the continuous phase as the matrix. Although the crystalline phase has a very high volume fraction, the continuous metallic glass is definitely considered to be the 
matrix. This character distinguishes other common composites with amorphous phases as the reinforcements $[54,55]$.

\subsubsection{Fiber-reinforced MGMCs}

In 1998, with the tungsten and carbon-steel continuous wire reinforcement, the $\mathrm{Zr}_{41.2} \mathrm{Ti}_{13.8} \mathrm{Cu}_{12.5} \mathrm{Ni}_{10.0} \mathrm{Be}_{22.5}$ matrix was quenched to a glass after infiltrating the reinforcement, and long-fiber reinforced MGMCs were firstly synthesized by Dandliker et al. [56, 57]. The SEM micrographs of an 80 vol. \% tungsten-wire reinforced composite are shown in Figure 6 [56]. The presence of continuous long fibers in the glass matrix can effectively retard the prompt development of shear bands upon loading, analogous to the case in particle-reinforced MGMCs. Note that the unreinforced metallic glass is elastic up to $1,900 \mathrm{MPa}$, and shows no significant plastic deformation. In contrast, the composite reinforced with steel wires has a plastic strain of over $2 \%$ and slightly improved ultimate strength, and the composite sample reinforced with tungsten wires also has an increased ultimate strength, in addition to a plastic strain of nearly $16 \%$ before failure [56].

Fiber-reinforced metallic matrix composites are widely found in $\mathrm{Al}, \mathrm{Mg}, \mathrm{Cu}$, and Ti alloys, which can increase their strengths. Here, for MGMCs with fibers as reinforcements, ductilization instead of strength enhancement is paid more attention. Up to now, there are $\mathrm{W}_{\mathrm{f}} / \mathrm{Zr}$-based [56-62], $\mathrm{W}_{\mathrm{f}} / \mathrm{Cu}$-based [63], Cu $\mathrm{f}_{\mathrm{f}} / \mathrm{Zr}$-based [64], $\mathrm{Ta}_{\mathrm{f}}$ / Zr-based [65], stainless-steel fiber / Zr-based MGMCs (the subscript, f, denoting fibers) [66]. For this kind of composites, the modulus of the composites is usually calculated, using the Rule of Mixture (RoM): 


$$
E_{c}=f E_{f}+(1-f) E_{m}
$$

where $E_{\mathrm{c}}, E_{\mathrm{f}}$, and $E_{\mathrm{m}}$ are the moduli for the composite, the fiber, and glass matrix, respectively, and $f$ is the volume fraction of fibers. According to Eq. (1), $E_{\mathrm{c}}$ is easily calculated, which is highly consistent with the experimental results [57]. Besides, some functional applications of continuous fiber-reinforced MGMCs may be achieved. For example, it has been demonstrated that copper-fiber-reinforced MGMCs are promising candidates for the applications where a direction-dependent conductivity is required to enhance the efficiency in the heat flow [64]. Wang et al. have found that the short irregular brass fibers can dramatically reduce the resistivity of the Ni-based BMG, leading to an improved material with both the high strength and good conductivity for functional applications [67].

Regardless of particle- or fiber-reinforced MGMCs, the wetting behavior has an important effect on the bonding at the interface [68]. The fiber-reinforced MGMC is taken as an example. The infiltration pressure, the volume fraction of fibers, and the filtration length together determine the reactive wetting. For a system in which the melt partly wets the fibers, the extra pressure, $P$, is needed to put the metallic melt to infiltrate into fibers. For the ideal unidirectional infiltration, the analytical solution of the filtration velocity, $v$, can be derived as [68]:

$$
v=\frac{R^{2}}{8 \eta h}\left[P+\rho g\left(H_{0}+h V_{f}\right)+\frac{2 \sigma_{\mathrm{lg}} \cos \theta}{R}\right]
$$

where $h$ is the length of fibers, $H_{0}$ the height of the melt, $R$ the radius of the fiber, $g$ the gravitational acceleration, $\eta$ the viscosity of the melt, $\rho$ the density of the melt, $\theta$ the 
contact angle, and $\sigma_{\mathrm{lg}}$ the surface tension of the melt. Based on Eq. (2), the determined infiltration-dynamics investigation is believed to provide the valid foundation for controlling preparation conditions of fiber-reinforced MGMCs.

\subsection{In-situ MGMCs}

The nature of plasticity improvement by introducing secondary phases in the metallic glass matrix is that the secondary phases can impede the rapid propagation of shear bands initiated in the matrix, and promote the formation of multiple shear bands. Furthermore, the localized shear bands can accommodate the plasticity. Except for the effective addition of ex-situ phases, in-situ crystalline phases during the solidification of melts may be another good selection, which could not only afford the plastic deformation within them, but also retard shear banding [69]. Usually, the in-situ crystalline phases mainly comprise nanocrystallites, dendrites, and crystalline phases, which undergo the martensitic transformation during plastic deformation. Besides, only a few special brittle intermetallic phases $[70,71]$ or in-situ two amorphous phases through the phase separation $[72,73]$ can toughen the BMGs. The gain of plasticity is attributed to the unique glassy structure correlated with the chemical inhomogeneity on the micron scale, the hard phases surrounded by the soft phases, leading extensive shear-band formation, interactions, and multiplication [72].

\subsubsection{Nanocrystalline ductilization}

Fan et al. found that the $\mathrm{Zr}_{53} \mathrm{Ti}_{5} \mathrm{Ni}_{10} \mathrm{Cu}_{20} \mathrm{Al}_{12}$ and $\mathrm{Zr}_{60} \mathrm{Cu}_{20} \mathrm{Pd}_{10} \mathrm{Al}_{10}$ as-quenched BMGs exhibited significant plastic strains $[74,75]$. High-resolution electron microscopy showed that nanocrystals with an average grain size of about $2 \mathrm{~nm}$ 
were embedded in the amorphous matrix of the two BMGs. The increased ductility was explained by the multiplication of shear bands due to the stress concentration in the vicinity of the nanocrystals $[74,75]$. Xing et al. found that $\mathrm{Zr}_{62-\mathrm{x}} \mathrm{Ti}_{\mathrm{x}} \mathrm{Cu}_{20} \mathrm{Ni}_{8} \mathrm{Al}_{10}(3<\mathrm{x}<$ 5) amorphous alloys crystallized via the precipitation of icosahedral quasicrystals in the primary crystallization step, leading to nano-sized quasicrystals embedded in an amorphous matrix [76]. Kim et al. found that $\mathrm{Ti}_{40} \mathrm{Zr}_{29} \mathrm{Cu}_{8} \mathrm{Ni}_{7} \mathrm{Be}_{16} \mathrm{BMGs}$ crystallized by forming a few 3-5-nm-sized quasicrystals with the volume fraction of about $7 \%$ in the amorphous matrix, enabling the fabrication of quasicrystal-reinforced MGMCs. The stable low-energy interface between the glass matrix and quasicrystals may act as a source for the multiple-shear-band formation, corresponding to plastic strains larger than $6 \%$ at room temperature [77]. Surprisingly, Inoue et al. found that the as-cast $\mathrm{Cu}_{50} \mathrm{Zr}_{50} \mathrm{BMG}$ can sustain a compressive plastic strain of more than $50 \%$ at room temperature, an exceptional value, which is explicable by the compensation of any shear softening through nanocrystalline coalescence and pinning of shear bands [78]. In a word, the improved plasticity is obtained for nanocrystallite-reinforced MGMCs. Experimentally, it is proposed the ductilization effect of nanocrystals in the MGMCs is from a strong interaction between nanocrystals and local shear bands during deformation [79]. Nanocrystals can grow in shear bands, delocalize the shear, and deviate and blunt cracks by the aid of in-situ deformation in the TEM analysis [80]. By simulations [81], features of the deformation morphologies of the composites are similar to those observed in experiments, including (1) the deformation initiated at the interfaces; (2) the bending of shear bands around crystallites, resulting in the 
propagation in an orientation away from the maximum shear direction; and (3) blockage of shear bands by crystallites. Several factors affect the overall mechanical behavior. The dominant effect is the presence of weak interfaces, which play a dual role in that they weaken the entire specimen and also provide multiple locations for shear-band initiation. A second factor is the resistance to the plastic deformation of the nanocrystallite, when a shear band tries to propagate through it. The third factor is the shear-induced nanocrystal growth. The newly-formed crystalline phase is much less likely to participate in further deformation, thus impinging on shear-band activities. Such participation is neither the result of heating due to mechanical work nor due to the presence of compressive strains.

In fact, not only the nanocrystallite can act as obstacles to impede the fast propagation of shear bands [74-82], but also the nanoscale heterogeneity in amorphous alloys could act as precursors for the formation of multiple shear bands [83-85]. Thus, shear banding is preferentially initiated at the interfaces or soft regions, and these shear bands can distribute the applied strain more homogeneously, resulting in the enhanced plasticity.

\subsubsection{Dendrite or Ta solid-solution ductilization}

In 2000, the first in-situ ductile-metal (dendrite)-reinforced MGMC based on glass-forming compositions in the $\mathrm{Zr}-\mathrm{Ti}-\mathrm{Cu}-\mathrm{Ni}-\mathrm{Be}$ system had been successfully developed by Hays et al. [86]. Primary dendrite growth and solute partitioning in the molten state yielded a microstructure consisting of a ductile crystalline Ti-Zr- $\mathrm{Nb} \beta$ phase, with a body-centered cubic (bcc) structure, in a $\mathrm{Zr}-\mathrm{Ti}-\mathrm{Nb}-\mathrm{Cu}-\mathrm{Ni}-\mathrm{Be}$ bulk 
metallic glass matrix, which resulted in a dramatic increase in the plastic strain to failure, impact resistance, and toughness of the metallic glass. This finding opens the possibility of producing an entirely-new class of high strength, tough, impact, and fatigue-resistant metallic materials, which combine the high strength of the metallic glass matrix with the ability to experience plastic deformation under unconfined or otherwise unstable loading conditions.

Figure 7 shows a dual-phase microstructure containing $\beta$-phase dendrites in a glass matrix, and an estimated fraction is about 25 vol. $\%$ of $\beta$ phases [86]. The inset of the XRD-identified peaks [labeled with (hkl) values] are due to the bcc phase. Upon cooling from the high-temperature melt, the alloy undergoes the partial crystallization by the nucleation and subsequent dendritic growth of the $\beta$ phase in the remaining liquid. In order to provide a deep understanding of the formation of such kinds of dual-phase composites, a pseudo-binary phase diagram for the in-situ $\beta$-phase-reinforced composites had been constructed by Lee et al. [87]. Figure 8 exhibits the processing map for the in-situ $\beta$-phase composites during a cooling experiment [87]. The Vit 1 alloy, $\left(\mathrm{Zr}_{75} \mathrm{Ti}_{25}\right)_{55}\left[\mathrm{Be}_{50}\left(\mathrm{Cu}_{55} \mathrm{Ni}_{45}\right)_{50}\right]_{45}$, and monolithic crystalline phase, $\mathrm{Zr}_{75} \mathrm{Ti}_{25}$, are selected as binary axes, and the in-situ composite, $\left(\mathrm{Zr}_{75} \mathrm{Ti}_{15} \mathrm{Nb}_{10}\right)_{75}\left[\mathrm{Be}_{50}\left(\mathrm{Cu}_{55} \mathrm{Ni}_{45}\right)_{50}\right]_{25}$, is marked by an arrow at $\mathrm{x}=0.424$, according to the lever rule from each solubility limit, based on the fact that the amorphous matrix occupies 60 vol. $\%$, and the $\beta$ phase takes up the remaining 40 vol. $\%$. The actual amorphous and crystalline phase compositions turn out to be $\mathrm{M}$ $\left(\mathrm{Zr}_{42.2} \mathrm{Ti}_{9.4} \mathrm{Nb}_{3.2} \mathrm{Cu}_{13.7} \mathrm{Ni}_{10.7} \mathrm{Be}_{20.8}\right)$ and $\mathrm{B}\left(\mathrm{Zr}_{71.1} \mathrm{Ti}_{13.1} \mathrm{Nb}_{13.4} \mathrm{Cu}_{1.6} \mathrm{Ni}_{0.8}\right)$, determined by 
the electron-microprobe analysis. M sits on $\mathrm{x}=0.076$ based on a Be content of 20.8 at. $\%$ as the amorphous matrix of the composite, compared with $22.5 \%$ Be in Vit 1 . The phase (B) occupies $x=0.947$ from $[(97.6-55.0) /(100-55)=0.947]$, according to the $\mathrm{Zr}_{75}\left(\mathrm{Ti}_{\mathrm{y}} \mathrm{Nb}_{1-\mathrm{y}}\right)_{25}$ overall content of $97.6 \%$. Note that Vit 1 , $\left(\mathrm{Zr}_{75} \mathrm{Ti}_{25}\right)_{55}\left[\mathrm{Be}_{50}\left(\mathrm{Cu}_{55} \mathrm{Ni}_{45}\right)_{50}\right]_{45}$, has $55 \%$ of $\mathrm{Zr}_{75}\left(\mathrm{Ti}_{\mathrm{y}} \mathrm{Nb}_{1-\mathrm{y}}\right)_{25}$, while the monolithic phase has $100 \% \mathrm{Zr}_{75}\left(\mathrm{Ti}_{\mathrm{y}} \mathrm{Nb}_{1-\mathrm{y}}\right)_{25}$. Figure 9 shows the SEM results, and the estimated volume fractions from the phase diagram are in good agreement with the measured volume fractions of the phase [87], as exhibited in Table 2 [87], and all show very good agreement between the phase diagram predictions and experimental values.

In addition, recently, a series of plastic Zr-based MGMCs have been designed, based on a pseudo ternary phase diagram with apexes of zirconium, (titanium + niobium), and $\mathrm{X}$, where $\mathrm{X}$ represents the moiety of $\mathrm{Cu}_{5} \mathrm{Ni}_{4} \mathrm{Be}_{9}[86,88]$, as displayed in Figure 10. Four compositions are on a line in the phase diagram, which can be written as $\left(\mathrm{Zr}_{75} \mathrm{Ti}_{25-\mathrm{x}} \mathrm{Nb}_{\mathrm{x}}\right)_{100-\mathrm{y}} \mathrm{X}_{\mathrm{y}}$. The $\mathrm{Zr}_{41.2} \mathrm{Ti}_{13.8} \mathrm{Cu}_{12.5} \mathrm{Ni}_{10} \mathrm{Be}_{22.5} \mathrm{BMG}$ (Vit 1), with the best glass-forming ability known so far in the $\mathrm{Nb}$-free $\mathrm{Zr}-\mathrm{Ti}-\mathrm{Cu}-\mathrm{Ni}-\mathrm{Be}$ system, lies on the same line with $\mathrm{x}=0$ and $\mathrm{y}=45$. Here, we fixes the value of $\mathrm{x}(\mathrm{x}=$ $6.66)$, and varies the value of $y(y=20,22,25$, and 28). Copper-mould suction casting, a cost-effective in-situ processing method, is employed to synthesize composites ( $3 \mathrm{~mm}$ in diameter), and the mechanical properties of these composites are studied.

Figure 11 shows the X-ray diffraction patterns of four composites with different compositions, labeled as Zr54, Zr56, Zr58, and Zr60, respectively, in Figure 
10) [88]. The detailed compositions of $\mathrm{Zr54}, \mathrm{Zr56}, \mathrm{Zr58}$, and $\mathrm{Zr60}$ are listed in Table 3. It is identified that the $\beta-\mathrm{Zr}$ phase with a bcc structure is detected, and its diffraction peaks are superimposed on the broad diffuse scattering maxima from the glass matrix. The crystal-plane indices of the $\beta$-Zr phase corresponding to diffraction peaks are marked in Figure 11. The intensity of the first peaks around 36 degree with the crystal-plane index of (110) is remarkably increasing from $\mathrm{Zr54}$, Zr56, and Zr58, to $\mathrm{Zr60}$, which indirectly demonstrates that the volume fraction of the $\beta-\mathrm{Zr}$ solid solution is increased. The differential scanning calorimeter (DSC) traces exhibit the endothermic characteristics of a glass transition, followed by multiple exothermic crystallization reactions at higher temperatures (not shown), further suggesting the glass nature of the matrix.

Figure 12 presents the SEM images of the cross sections of the developed composites [88]. For the Zr54 alloy, very few dendrites are distributed in the glass matrix, as shown in Figure 12(a), which is consistent with a low intensity of the $\beta-\mathrm{Zr}$ phase peaks detected in the XRD pattern of Figure 11. For the Zr56 alloy, the dendrites with a very fine microstructure possess a volume fraction of about $50 \%$, as displayed in Figure 12(b). Figure 12(c) exhibits a well-developed flowery dendritic morphology for the Zr58 alloy, and the characteristic spanning length of an individual dendrite is about $20 \mu \mathrm{m}$. The flowery dendrites, with a volume fraction of about $55 \%$ for the Zr58 alloy, are homogeneously distributed in the glass matrix. A typical dendritic morphology develops in the glass matrix in the Zr60 alloy, as illustrated in Figure 12(d), in which the volume fraction of the dendrites is about $60 \%$, and the 
primary dendritic arm has a diameter of $1-2 \mu \mathrm{m}$. As designed above, the slight tuning of atomic ratios dramatically changes the microstructure, which gives a reminder that we should strictly control the chemical compositions of glass-forming alloys to avoid the full crystallization of glass matrices.

The compressive engineering stress-strain curves of designed alloys at room temperature are displayed in Figure 13(a) [88]. The yielding strength $\left(\sigma_{y}\right)$, the yielding strain $\left(\varepsilon_{\mathrm{y}}\right)$, the ultimate strength $\left(\sigma_{\max }\right)$, and the fracture strain $\left(\varepsilon_{\mathrm{f}}\right)$ of these composites together with mechanical properties of Vit 1 are summarized in Table $3[88,89]$. Compared with the fracture strain of Vit 1 , all the four composites are markedly improved, due to the transfer of the local stress concentration to the surrounding glass, when the soft $\beta-Z r$ phase yields upon loading [86], especially for the Zr60 alloy with a fracture strain of $17.0 \%$. All these composites exhibit the work-hardening behavior during plastic flows. The tensile engineering stress-strain curve of the $\mathrm{Zr60}$ composite is shown in Figure 13(b) [90]. The ultimate tensile strength is about 1,480 MPa, and the tensile fracture strain attains $2.9 \%$. Generally, BMGs and MGMCs exhibit softening under tensile loading at room temperature, even though they display a large compressive fracture strain [86-90]. During the past 15 years, there are many such dendrite / MGMCs successfully designed, which exhibit the macroscopic ductility and high strength at room temperature, together with the high thermal stability of the glass matrix [91], which include the dendrite / Zr-based [86-90, 92-97], dendrite / Ti-based [98-105], dendrite / Co-based [106], dendrite (flake) / Mg-based [107], dendrite / 
La-based [108, 109], dendrite / LaCe-based [110], and dendrite / Fe-based MGMCs $[111,112]$

In in-situ ductile crystalline-reinforced composites, the characteristic morphology of crystalline phases is mainly dendritic, and the bec dendritic $\beta-\mathrm{Zr}$ and $\beta$-Ti solid solutions are most popular in Zr- and Ti-based MGMCs. Moreover, in-situ Ta particle-reinforced MGMCs are widely recognized. The Ta particles are more ductile than the glass matrix. As early as in 2002, Fan et al. had discovered precipitated micron-scale Ta-rich solid-solution particles distributed in a metallic glass matrix during melting of the master alloy of the glass-forming $\left(\mathrm{Zr}_{70} \mathrm{Ni}_{10} \mathrm{Cu}_{20}\right)_{82} \mathrm{Ta}_{8} \mathrm{Al}_{10} \mathrm{BMG}$ [113]. The microstructure of the as-cast composite samples is shown in Figure 14 [113]. Particles of the Ta-rich solid solution, with an average size of $10-30 \mu \mathrm{m}$ and volume fraction of about $4 \%$, are dispersed throughout the glass matrix. The particles are oblong in shape and do not appear to possess a dendritic morphology and an average chemical composition of the crystalline particles is $\mathrm{Ta}_{93.2} \mathrm{Zr}_{5.4}(\mathrm{Cu}+\mathrm{Ni}+\mathrm{Al})_{1.4}$.

For this kind of composites, in contrast to dendrite composites, the differences between them are manifested by not only the morphology, but also the chemical constitute. In the developed dendrite composites mainly, including Zr- and Ti-based BMG matrix, the $\beta$ solid solutions are composed of $\mathrm{Zr}, \mathrm{Ti}$, and $\mathrm{Nb}$ for the Zr-based matrix, Ti, Zr, and V for the Ti-based matrix, and Zr and / or Ti occupy the most atomic percents. During solidification, $\mathrm{Nb}$ and / or $\mathrm{V}$ are partitioned into both matrices and $\beta$ solid solutions, and they are as a stabilizer, favoring the formation of 
bcc solutions $[100,114]$. Analogous formation has been widely known in crystalline $\mathrm{Zr}$ and Ti alloys $[115,116]$. However, in in-situ Ta-reinforced composites, only a few elements can dissolve into bcc Ta particles [113, 117, 118]. Ta has a melting temperature near 3,000 degrees, and tends to precipitate prior to other elements with lower melting temperatures upon cooling. With the dramatical decrease of the processing temperature, it is difficult to diffuse along the interface between the melts and Ta particles for other glass-forming elements, and Ta-rich particles form. Similarities between the elementary Ta and $\mathrm{W}$ have been found in the design of in-situ $\mathrm{W}_{\mathrm{p}}$ / MGMCs [119]. Although the differences between dendrites and Ta particles obviously exist, the function of these two reinforcements during plastic deformation is the same. These Ta-rich particles serve more than as traditional force carriers in the composites but as the main obstructions for the shear-band propagation $[113,117,118,120,121]$. Finite-element analysis (FEA) demonstrates that homogeneously-dispersed Ta-rich particles form a network in the matrix to effectively localize shear banding and, thus, avoid catastrophic failures [121]. The deformation behavior of the dendrite or Ta particle / MGMCs would be investigated in detail in Section 3 .

\subsubsection{Transformation-mediated ductilization}

It has been demonstrated that a diffusionless phase transformation during plastic deformation can significantly enhance the ductility / toughness of different crystalline materials [122], known as the transformation-induced plasticity (TRIP) effects. As the tensile strength increases, conventional steels show a decrease in 
elongation to failure, which is a measure of formability; that is, the higher its strength, the less formable the steel is. Dual-phase (DP) and TRIP steels deviate from this general rule by combining the high strength with the significantly-higher elongation values than those of conventional steels of the same strength level. Specially in TRIP steels, a certain amount of metastable-retained austenites can transform into martensites upon mechanical loading. During plastic flows, the improved toughness can be achieved, accompanied by the obvious work hardening. If this concept can be applied to the fabrication of in-situ BMG composites, a new kind of MGMCs could be obtained, which combine the high strength with the macroscopic ductility via the phase transformation upon mechanical loading.

Recently, this kind of composites has been successfully synthesized by different research groups, and the distinguished work hardening was achieved regardless of tensile or compressive loadings [123-133]. The parent phase has a size of about several microns, similar to that of dendrites or Ta-particles and larger than that of nanoprecipitates within the amorphous matrix [74-78, 134]. Up to now, the discovered parent phase in the metallic glass matrix is the equiatomic $\mathrm{CuZr}$ intermetallic compound [123-133], which exhibits the same shape-memory effect as $\mathrm{Ni}-\mathrm{Ti}$ and $\mathrm{Cu}-\mathrm{Zn}-\mathrm{Al}$ alloys [135]. The equiatomic $\mathrm{CuZr}$ phase has different metastable structures, i.e., the high-temperature $\mathrm{B} 2 \mathrm{CuZr}$ phase with a cubic primitive structure (Pm-3m), and monoclinic martensites $\left(\mathrm{P} 2_{1} / \mathrm{m}\right.$ and $\left.\mathrm{Cm}\right)$, as schematically shown in Figure $15[136,137]$. Meanwhile, the lattice parameters of the basic structure and of the superstructures of the martensites are $a=0.3278 \mathrm{~nm}, b=0.4161 \mathrm{~nm}, c=0.5245$ 
$\mathrm{nm}$, and $\beta=103.88^{\circ}$, and $a=0.6316 \mathrm{~nm}, b=0.8562 \mathrm{~nm}, c=0.5331 \mathrm{~nm}$, and $\beta=$ $105.27^{\circ}$, respectively [136].

During plastic deformation or cooling, the $\mathrm{B} 2 \mathrm{CuZr}$ phase transforms into martensites, and is not stable at room temperature for most of CuZr-based alloys. For example, the $\mathrm{B} 2 \mathrm{CuZr}$ phase can exist in equilibrium above $988 \mathrm{~K}$ [137], and below it, the $\mathrm{B} 2 \mathrm{CuZr}$ phase decomposes into the low-temperature equilibrium phases (LT-EPs), such as $\mathrm{Cu}_{10} \mathrm{Zr}_{7}$ and $\mathrm{CuZr} r_{2}$. However, undergoing rapid quenching, this eutectoid decomposition may be avoided, and, thus, the $\mathrm{B} 2 \mathrm{CuZr}$ phase may be reserved to room temperature [137]. Figure 16 shows a schematic of the continuous cooling transformation (CCT) diagram for the $\mathrm{Cu}_{50} \mathrm{Zr}_{50}$ alloy [137]. It can be seen that two important factors have to be considered for the formation of the CuZr-based MGMCs, i.e., the formation of the amorphous phase and the $\mathrm{B} 2 \mathrm{CuZr}$ phase. On one hand, the formation of the amorphous phase is usually determined by the stability of the liquid phase and its resistance to crystallization [138]. On the other hand, during quenching, the supercooled liquid competes with the $\mathrm{B} 2 \mathrm{CuZr}$ phase and the low-temperature equilibrium phases (LT-EPs). When the cooling rate is higher than the critical cooling rate for the full glass formation $\left(R_{\mathrm{c}}\right)$, only the amorphous phase is observed. In contrast, the LT-EPs are obtained under much lower cooling rates. In such a case, an appropriate cooling rate is required in order to obtain the $\mathrm{B} 2 \mathrm{CuZr}$ phase and the amorphous phase simultaneously. Therefore, the competition among the metastable B2 phase, the amorphous phase, and the LT-EPs during quenching is the dominant factor for the formation of CuZr-based MGMCs, as shown in Figure 16. 
The formation of the CuZr-based MGMCs is highly dependent on the location and the shape of the "nose" of the competing phases in the CCT diagram. This trend means that the processing cooling rate must fall between the critical cooling rate for the full glass formation, $R_{\mathrm{c}}$, and the critical cooling rate for the $\mathrm{B} 2$ phase formation, $R_{\mathrm{b} 2}$, in order to obtain both the $\mathrm{B} 2 \mathrm{CuZr}$ phase and the amorphous phase.

After the formation mechanisms are well understood, the mechanical properties could be controllably tailored by tuning the microstructure. Figure 17 presents the variation of the tensile ductility, $e_{\mathrm{T}}$, as a function of the crystalline fraction, $V_{\mathrm{c}}$, in the $\mathrm{Cu}_{47.5} \mathrm{Zr}_{47.5} \mathrm{Al}_{5}$ MGMCs [132]. More than the tensile ductility of 10 $\%$ can be reached by tailoring the microstructure. It turns out that $e_{\mathrm{T}}$ exhibits a unimodal hump-shaped relation with $V_{\mathrm{c}}$. It achieves its maximum value in the crystallinity range of about $40 \%-70 \%$ and decreases steeply on both sides of the plateau. Recent studies have indicated that there exists a topological transition in a statistically-critical microstructural condition for this kind of MGMCs, where the microstructure is the most effective in hindering the propagation of shear bands [95, $102,108]$. This transition can be termed as the percolation and the transition point as the percolation threshold. The relationship between $e_{\mathrm{T}}$ and $V_{\mathrm{c}}$ can be modeled quantitatively according to the percolation theory [139]:

$$
e_{T} \propto\left(V_{c}^{p}-V_{c}\right)^{-\beta}
$$

where $V_{c}^{p}$ is the percolation threshold or the critical crystalline volume fraction to generate the microstructural percolation, and $\beta$ is a power exponent [139]. The calculation is well consistent with the experimental result, when the parameters, $V_{c}^{p}$ 
and $\beta$, are quantified as $55 \%$ and 2 , respectively. The percolation threshold has been successfully achieved in the transformation-mediated MGMCs.

In addition, the mechanical properties can be tailored by varying the microstructure via adding minor elements. Song [137] has systematically investigated eleven CuZr-based alloy systems, altogether 36 different compositions. Even if the addition of minor elements is operated, the microstructure and mechanical properties are greatly affected. Wu et al. found that the twinning property of the reinforcing crystals could be dramatically improved by reducing the stacking fault energy through microalloying, effectively altering the electron-charge density redistribution on the slipping plane [130]. Notably, the Co substitution of $\mathrm{Cu}$ in the $\mathrm{B} 2 \mathrm{CuZr}$ structure drastically reduces the electron-charge-density redistribution. Consequently, a minor addition of Co can remarkably improve the tensile ductility with a value of about $7 \%$ and work-hardening capability of MGMCs. The implications of the transformation-mediated ductilization are important for developing high-performance MGMCs.

\subsection{Fabrication of bulk metallic glasses and composites}

\subsubsection{Fabrication of monolithic BMGs}

As early as in 1960, Au-Si binary amorphous alloys have been successfully quenched via high cooling rates [1]. Later, amorphous alloys with ribbon or strip types were mainly developed by melt spinning [5]. Until later in 1970s, some BMGs with low critical cooling rates have appeared. For example, Drehman et al. synthesized $\mathrm{Pd}_{40} \mathrm{Ni}_{40} \mathrm{P}_{20}$ BMGs with a slow cooling rate of $1.4 \mathrm{~K} / \mathrm{s}$ on a fused silica 
surface under $10^{-6}$ Torr vacuum [4]. Coming into 1990s, in Inoue's group, in order to produce BMGs with a thickness above $10 \mathrm{~mm}$, copper-mold suction casting [9], water quenching of a melt in a quartz tube [8], and unidirectional zone melting by the usage of arc melting as a heat source [140] were widely employed. Up to now, copper-mold suction casting is the most common technique to fabricate BMGs, and the most developed BMGs are based on this method all over the world.

Figure 18 exhibits a typical schematic illustration of copper-mold suction casting. The suction-casting system consists of two chambers: an upper chamber, in which the alloy ingot is remelted, and a lower chamber, which holds a copper mold. The mold and the arc-melting hearth are connected by a round orifice, which has a diameter of about $1 \mathrm{~mm}$. The lower chamber is connected to a vacuum reservoir through a pneumatically-actuated valve. To cast a sample, an ingot of the desired composition is melted under an Ar. While the ingot is molten, the valve is opened, creating a vacuum in the mold chamber and sucking the molten alloy into the mold. As a result, the rod and plate samples or samples with even more complex shapes can be fabricated by designing the inner shape of the mold. Other types of copper-mold-suction-casting systems are the same in essentials, while different in minor points. For instance, from the arc melter in Liaw's group from The University of Tennessee, as shown in the inset in Figure 18 [141], it can be seen that the four-piece suction-casting die is sized to seat in the hearth leaving a $0.15-\mathrm{mm}$ gap between the upper (crucible) and lower (mold) portions. Moreover, the die was split into upper and lower portions in order to minimize the heat flow into the casting 
mold. With the development of the large-sized monolithic BMGs, all kinds of new methods come into play, including the spark-plasma sintering [142], electromagnetic-vibration process [143], pulsed-laser forming [144], low-pressure die-casting [145], melt atomization and spray deposition [146], continuous casting [147], etc. Any routes, which as long as could "beat" the crystallization in melts by processing, would be popular in both the scientific research and technology fields. People have been always searching for strategies to optimize the GFA by computer simulations [148-150] and / or employing advanced ways to pinpoint GFA [151, 152]. Very recently, Ding et al. [152] proposed a high-throughput strategy. Using this approach, the composition with the highest thermoplastic formability in the glass-forming system of $\mathrm{Mg}-\mathrm{Cu}-\mathrm{Y}$ is quickly identified. The method provides a versatile toolbox for unveiling complex correlations of material properties and glass formation, and should facilitate a drastic increase in the discovery rate of metallic glasses. Despite an increasing theoretical understanding of glass formation, BMGs are predominantly developed through a sequential and time-consuming trial-and-error approach.

\subsubsection{Fabrication of ex-situ MGMCs}

As described before, as early as in 1997, ex-situ particle-reinforced Zr-, and $\mathrm{Cu}$-based MGMCs have been successfully synthesized by melt infiltration using an induction heating coil and, then, injected melts through a nozzle into a copper mold [37]. Figure 19 shows the schematic diagram of the apparatus [37]. In this method, the top-fill casting-liquid metal is forced downward by a pressurized gas into a preform, 
and the particle-reinforced and fiber-reinforced MGMCs can be easily synthesized. Actually, this technique has been extensively employed to synthesize metal and ceramic matrix composites $[153,154]$. As the case of copper-mold suction casting to obtain BMGs, melt infiltration is the most popular in the fabrication of ex-situ MGMCs [37-53]. During melt infiltration, the extra pressure, the infiltration temperature, and melt / fiber interface have an important effect on the infiltration kinetics. Equation (2) imparts the quantitative analysis of the effect of the extra pressure. The simple theoretical treatments are given as follows.

To follow the temperature evolution during infiltration, a representation of the thermal behavior of both the fibers and melt is required as well as for the combined composite. The enthalpy $(H)$ of the composite can be derived via Eq. (4) [155]:

$$
H=\int_{298}^{T}\left(M_{f} C_{f}+M_{m} C_{m}\right) d T+\left(1-f_{s}\right) M_{m} G_{m}
$$

where $M$ is the mass fraction, $C$ the specific heat, $f_{\mathrm{s}}$ the matrix solid fraction, and $G$ the heat of fusion. The subscript, $f$, denotes the fiber and $m$ the matrix. In the case of the melt, a fixed melting point is used, at which the state change (e.g., phase transformation) occurs, leading to a large step in the resultant $H(T)$ function. The fiber is assumed not to undergo state changes, such as phase transformation, and, hence, has no step in the $H(T)$ function. The functions for the melt and matrix components can, hence, be combined to produce that of the composite, using the mass fractions of the fibers and melt ( $M_{\mathrm{f}}$ and $M_{\mathrm{m}}$, respectively). 
Moreover, the dual-phase boundary is of critical importance in the consideration of the thermal evolution during infiltration. A concentric fiber / melt cell, consisting of the appropriate melt volume for the fiber radius $\left(R_{\mathrm{f}}\right)$ and fiber volume fraction $\left(V_{\mathrm{f}}\right)$, may be introduced for the consideration of heat transfer on the scale of a single fiber, the outer cell $\left(R_{\mathrm{c}}\right)$ radius given by the following Eq. (5) [155].

$$
R_{c}=\frac{R_{f}}{\sqrt{V_{f}}}
$$

Using the one-dimensional axisymmetry of a cell containing a single central fiber, surrounded by the appropriate volume of the melt given by the fiber volume fraction $\left(V_{\mathrm{f}}\right)$, it is possible to simply follow the heat transfer with time. The effect of the heat-transfer coefficient across the interface was considered. Based on Eqs. (4) and (5), the effects of the infiltration temperature and melt / fiber interface on fabrication can be quantitatively analyzed, which reminders people of optimizing the ex-situ MGMCs processing.

\subsubsection{Fabrication of in-situ MGMCs}

During the past two decades, considerable research efforts have been directed towards the development of in-situ metal matrix composites (MMCs), in which the reinforcements are formed in-situ by the exothermal reactions between elements or between elements and compounds [156]. Using this approach, MMCs with a wide range of matrix materials (including aluminum, titanium, copper, nickel and iron), and second-phase particles (including borides, carbides, nitrides, oxides, and their mixtures) have been produced. 
Contrary to the conventional in-situ MMCs, the ductile phase is the secondary phase in in-situ MGMCs, specially for dendrites and Ta solid solutions, rather than the metallic glass matrix. These secondary phases play an important role in the ductilization of monolithic BMGs. The high strength originates from the network structure of the amorphous matrix. Most in-situ dendrite or Ta-particle-reinforced MGMCs are obtained by copper-mold suction casting. From the first in-situ dendrite composites $[86,89]$, people have noticed the heterogeneous distribution of secondary phases from the outer surface to the center of synthesized samples [88, 114, 157-159]. Kong et al. [157] has found that the secondary dendrite arm spacing (SDAS) closely depends on the cooling rate, and an increased SDAS dominates from the outer surface to the center. Even if the composition is fixed, different microstructures, including the size and volume fraction, could be obtained, related to the different cooling rates, using different-sized molds [160]. Utilizing the copper-mold suction-casting method, irreproducible microstructures prevail, resulting in irreproducible mechanical properties [160], which limits the industrialization of such kinds of high-performance composites. For the synthesis of the homogeneous in-situ MGMCs, copper-mold casting has not been the top priority yet. Therefore, several strategies mentioned below have been proposed to solve this stubborn problem (inhomogeneous microstructures), and homogeneous in-situ MGMCs have been obtained.

1. Semi-solid processing

In 2006, an innovative method was developed, based on the controlled solidification in the liquid-solid dual-phase region to produce spherical crystalline 
particles homogeneously dispersed in the amorphous matrix [161]. Firstly, alloy pieces sectioned from the ingots were encapsulated in quartz tubes in a high vacuum and heated to a temperature above their melting point. Secondly, the melts were, then, cooled to the temperature inside the liquid-solid dual-phase region, and held at that temperature for about $5 \mathrm{~min}$. At last, cylindrical samples with a diameter of $8 \mathrm{~mm}$ were prepared by quenching the molten alloy inside the quartz tube into the iced water. The synthesized composites by this semi-solid processing method exhibit the improved toughness. The spheroidization makes the crystals coarsen, which can more effectively increase cracking resistance [161-163]. Later, the ductile spherical crystal / metallic glass has been found in our group [162]. Semi-solid processing has been recognized as a good candidate to fabricate in-situ MGMCs with uniform microstructures.

Another exciting example to produce uniform in-situ composites by semi-solid processing happens in 2008 [94, 100, 164, 165]. This strategy, developed at Caltech, is carried out, using a water-cooled copper boat, diagrammed in Figure 20(a). An ingot is heated, while resting in an indentation boat with a water-filled copper tube. The sample is isolated in a Ti-gettered argon environment by a quartz tube and is heated by an induction coil. The high thermal conductivity of copper prevents the destruction of the boat, while the radio-frequency stirring and levitation homogenize the sample. A picture of equipment is shown in Figure 20(b). Overall, the technique route is refered to be the following features: (1) selecting a highly-processable glass-forming system with a large GFA, and finding dendrites that 
form in equilibrium with the glass-forming liquid, which have a lower shear modulus than the resulting glass; (2) processing the dual-phase alloys between the solidus and liquidus temperatures, which will coarsen the dendrites, create a homogeneous microstructure, and match the length scale of dendrites with the plastic zone size of the amorphous matrix; and (3) quenching rapidly to vitrify the remaining liquid and form a dual-phase composite consisting of an amorphous matrix with ductile secondary phases. In this design route, it is key to make sure that dendrites have lower shear moduli than glass matrices in order to obtain the macroscopic tensile ductility, since shear bands, initiated in plastically-soft regions with a lower shear modulus, $G$, can be arrested in surrounding regions of higher yielding stresses or stiffnesses. RoM can be employed to predict the $G$ value of dual-phase composites here, as shown in Figure 21(a) [94]. The glass matrix has a higher shear modulus ( $33 \mathrm{GPa})$ than the bcc dendrite $(\sim 28 \mathrm{GPa})$, indicating that the dendrite is a soft inclusion. Calculating the volume fraction of the glass by RoM yields $56 \%$, in excellent agreement with the image analysis and DSC scans [94]. Consequently, in-situ dendrite composites with a uniform microstructure by semi-solid processing, as shown in Figure 21(b). By the extension of semi-solid processing, thermoplastic processing [166] and semi-solid forging [164] are well developed to produce near-perfect replications and net shapes.

\section{Bridgman solidification (unidirectional solidification)}

Bridgman solidification is widely employed to synthesize conventional crystalline alloys, since the microstructure can be controlled by tailoring the temperature gradient and withdraw velocity [167]. By varying the withdrawal 
velocities in a controlled manner, the alloys with tailorable volume fractions and sizes of crystalline phases are obtained, owing to the variation of the cooling rates.

Furthermore, the distribution of the crystalline phase is still homogeneously attributed to the availability of the invariable cooling rates, since the direction of the thermal conduction and extraction is mainly along the longitudinal direction for rod-shape samples obtained by the Bridgman solidification. The early study on the formation of MGMCs by the Bridgman solidification was mainly carried out in La-based alloy systems, and precipitated $\alpha$-La dendrites were as the reinforcement $[18,109$, 168-170]. Since the La-based alloys have the intrinsic low strength, even if the optimal experimental parameters be chosen, only hundreds of MPa of the strength is achieved [109].

Later, significantly-ductile high-strength Zr-based [69, 95, 158, 162, 171-173] and Ti-based composites [102, 174, 175], consisting of amorphous phases and ductile bcc dendrites, came forth using the Bridgman solidification by Qiao et al. The Bridgman solidification apparatus used by Qiao et al. is shown in Figure 22(a), and the illustration is exhibited in Figure 22(b), which consists of an induction coil, a quartz tube, a heat insulator, a graphite heater, and a liquid Ga-In-Sn alloy with a water-cooled bath. The graphite heater was heated by an induction coil to melt the alloy under an argon atmosphere, and it can shield an electromagnetic field from an induction coil. The temperature was measured by W/Re thermocouples, and the temperature gradient $\left(G_{\mathrm{T}}\right)$ could be attained. After heating to the required temperature and holding for several minutes, a sample was directionally solidified into the liquid 
Ga-In-Sn alloy with a withdraw velocity of $\mathrm{v}$. The cooling rate $\left(R_{\text {cooling }}\right)$ could be calculated, according to the equation of $R_{\text {cooling }}=G_{\mathrm{TV}}$.

Figure 23(a) [69] displays the XRD patterns of the $\mathrm{Zr}_{37.5} \mathrm{Ti}_{32.2} \mathrm{Nb}_{7.2} \mathrm{Cu}_{6.1} \mathrm{Be}_{17.0}$ composites fabricated by the Bridgman solidification with different velocities, $\mathrm{v}=0.2-1.5 \mathrm{~mm} / \mathrm{s}$. It can be seen that the peaks for the bcc $\beta-\mathrm{Zr}$ phases are superimposed on the broad diffuse-scattering amorphous maxima, when the $\mathrm{v}$ increases to $0.5 \mathrm{~mm} / \mathrm{s}$ or higher, and the crystal-plane indices of the $\beta$-Zr phase corresponding to peaks are marked. For the sample with $\mathrm{v}=0.2 \mathrm{~mm} / \mathrm{s}$, not only the peaks for the $\beta-\mathrm{Zr}$ phase but also additional peaks (not indexed) corresponding to other crystalline phases are observed. The DSC results for $\mathrm{Zr}_{37.5} \mathrm{Ti}_{32.2} \mathrm{Nb}_{7.2} \mathrm{Cu}_{6.1} \mathrm{Be}_{17.0}$ composites are shown in Figure 23(b) $[69,95]$. The samples with $v \geq 0.5 \mathrm{~mm} / \mathrm{s}$ exhibit a glass transition, followed by a single exothermic event during continuous heating, which is an indicator of an eutectic crystallization event. If the composition of the metallic glass matrix is off eutectic, the multi-step exothermic events can be observed [91]. However, no glass transition and exothermic event are observed for the sample with $\mathrm{v}=0.2 \mathrm{~mm} / \mathrm{s}$, indicating that this composite is only composed of the crystalline phases. The glass-transition temperature $\left(T_{g}\right)$, the onset crystallization temperature $\left(T_{x}\right)$, and the integrated heat of crystallization $\left(\Delta H_{x}\right)$ of the samples are summarized in Table $4[69,95]$. From Table 4 , it can be seen that $T_{g}$ and $T_{x}$ do not change sharply. The heat of crystallization in these composites relative to the heat of crystallization in the matrix alloy is an estimation of the volume fraction of the glass [94]. It has been demonstrated that the compositions of matrixes in DH-series alloys 
(Zr-Ti-Cu-Be-Nb system) are similar [94]. Therefore, the analysis of the heat of crystallization of the glass matrix of the composites compared with that of the matrix of DH1 alloy $\left(\mathrm{Zr}_{36.6} \mathrm{Ti}_{31.4} \mathrm{Nb}_{7} \mathrm{Cu}_{5.9} \mathrm{Be}_{19.1}\right)\left(\Delta H_{x}=100.90 \mathrm{~J} / \mathrm{g}\right)$ gives a direct estimate of the volume fractions of $\beta-\mathrm{Zr}$ dendrites to be $64 \%, 43 \%, 42 \%$, and $40 \%$ corresponding to $\mathrm{v}$ of $0.5,0.8,1.0$, and $1.5 \mathrm{~mm} / \mathrm{s}$, respectively.

The microstructure of the sample with $\mathrm{v}=1.0 \mathrm{~mm} / \mathrm{s}$ is illustrated in Figure 24(a) [95]. It can be seen that dendrites homogeneously disperse in the glass matrix. An individual dendrite tree is indicated in the inset of Figure 24(a), which has a spanning length, $\mathrm{s}(\mu \mathrm{m})$, of about $330 \mu \mathrm{m}$. The dependences of spanning lengths of individual dendrite trees on varied withdrawal velocities are shown in Figure 24(b) [95], which roughly obeys a linear relationship. The relationship between $\mathrm{s}$ and $\mathrm{v}$ is approximately expressed by the following equation.

$$
s=-239 v+588
$$

By controlling the withdrawal velocities, $v$, the sizes of dendrites have been effectively tailored, which verifies that the microstructures of the composites can be tuned by the Bridgman solidification even when the composition is fixed. In addition, with the increase of withdrawal velocities, i.e., the increase of cooling rates, the growth of dendrites is suppressed, leading to the decrease of volume fractions of dendrites. Figure 24(c) plots the dependences of the fracture strengths and the plastic strains of the composites developed by the Bridgman solidification on v. It is noted that the mechanical data are collected from Ref. [95]. All the composites by the Bridgman solidification have a high fracture strength ( $>1,700 \mathrm{MPa})$ and exhibit the 
large plasticity. The composite with $\mathrm{v}=1.0 \mathrm{~mm} / \mathrm{s}$ combines the highest fracture strength $(\sim 3,000 \mathrm{MPa})$ and the largest plastic strain ( $>30 \%)$ [95]. In contrast to the composites with a diameter of $3 \mathrm{~mm}$ by copper-mold-suction casting, from the compressive engineering stress-strain curves of these composites (unpublished data), the yielding strength and the yielding strain are $\sim 1,670 \mathrm{MPa}$ and $\sim 2.0 \%$, respectively, while the fracture strength and the fracture strains are $\sim 2,070 \mathrm{MPa}$ and $\sim 4.0 \%$ for the samples fabricated by the Bridgman solidification, respectively. The bended sample is exhibited in the inset in Figure 24(c). The crack does not happen during bending until the bending angle is approaching 130 degree. It demonstrates that the Bridgman solidification is an advanced method to adjust and optimize the mechanical properties of the in-situ MGMCs.

Until now, both the semi-solid processing and Bridgman solidification may yield a uniform "near-equilibrium" dual-phase microstructure throughout the samples, leading to the improved ductility. The ductility of composites is tailored by designing a series of proper compositions, when semi-solid processing is selected. Through tuning the compositions of the composites, different scaled and volume-fractioned dendrites in the glass matrix can be obtained, and the enhanced ductility and toughness can be achieved. However, the ductility can't be tailored for the composites with the same composition by semisolid processing, since the cooling rates are fixed. Comparably, the ductility could be definitely tuned by the Bridgman solidification regardless of the fixed or tuned compositions in the composites.

\subsection{Summary}


This section clarifies the development history of MGMCs. According to the reinforcement phases in the glass matrix, the classification of MGMCs is schematically illustrated in Figure 25, based on the development of MGMCs until now. Artificially introducing ex-situ reinforcements with high melting temperatures into the glass-forming melts makes ex-situ MGMCs. Spontaneously, the partial crystallization of glass-forming melts elicits in-situ MGMCs. Specially, more advanced methods to fabricate homogeneous in-situ MGMCs are in expectation, other than the semi-solid processing and Bridgman solidification. 


\section{Deformation behavior of bulk metallic glasses}

\subsection{Free-volume model}

The classical free-volume theory was originally developed by Turnbull and Cohen to describe the molecular transport in glass-forming liquids [176, 177]. In 1977, Spaepen further developed this theory and established the free-volume model of the plastic flow in metallic glasses [178]. This model essentially views the basic "flow event" as an individual atom jump that is driven by the shear stress and assisted by thermal fluctuations, as schematically shown in Figure 26. A series of atomic jumps result in the free-volume creation that eventually contributes to the plasticity of glasses.

Within this physical picture, Spaepen constructed the constitutive model that uses the free volume, $v_{f}$, as the internal state variable of the system. The macroscopic plastic-strain rate, $\dot{\gamma}^{p}$, can be expressed by the following equation [178]:

$$
\dot{\gamma}^{\mathrm{p}}=\exp \left(-\frac{\chi v^{*}}{v_{f}}\right) 2 f \exp \left(-\frac{\Delta G^{m}}{k_{B} T}\right) \sinh \left(\frac{\tau \Omega}{2 k_{B} T}\right)
$$

The first term on the right-hand side accounts for the probability that an atom is on the potential jump site. The remaining terms describe the frequency (or the activation rate) of atomic jumps. In this equation, $\chi$ is a geometrical factor, $v^{*}$ is a critical volume that permits an atomic jump, $f$ is an attempt frequency, $\Delta G^{m}$ is the activation energy, $k_{B}$ is the Boltzmann constant, $T$ is the temperature, $\tau$ is the applied shear stress, and $\Omega$ is the atomic volume. By considering a dynamic competition between the stress-driven creation and diffusion-mediated annihilation, Spaepen derived the time evolution of free volumes as: 


$$
\dot{v}_{f}=v^{*} f \exp \left(-\frac{\chi v^{*}}{v_{f}}\right) \exp \left(-\frac{\Delta G^{m}}{k_{B} T}\right)\left\{\frac{2 \chi k_{B} T}{v_{f} S}\left[\cosh \left(\frac{\tau \Omega}{2 k_{B} T}\right)-1\right]-\frac{1}{n_{D}}\right\}
$$

where $n_{D}$ is the number of diffusive jumps necessary to annihilate a free volume equal to $v^{*}$, and $S$ is the Eshelby modulus.

It is well known that the diffusive-like atomic jump is usually driven by the pressure or normal stress gradient. However, the atomic jump in the free-volume model is shear-stress-driven. A possible reason stems from the shear-induced dilatation effect inherent to metallic glasses [179-181]. Such dilatations would invoke the local pressure within glasses, which would drive atomic diffusion and accompany the free-volume evolution. The Spaepen's free-volume model presents a relatively-systematic theoretical framework to the problem of the glass flow, which points out a direction into the fundamental understanding of the glass-flow mechanism through atomistic defects.

Since it is proposed by Spaepen [182], the free-volume model has been extensively developed and widely applied. For example, within the framework of the free-volume model, Johnson [182] developed a self-consistent dynamic free-volume model for describing the uniform steady-state flow in metallic glasses and deeply-undercooled glass-forming liquids. Recently, the free-volume model has also been developed to allow for large deformations [183], coupled thermomechanical deformations [184, 185], and even the localization process, i.e., shear banding [186-188], of plastic flows in metallic glasses.

\subsection{Shear-transformation zone (STZ) model}


Inspired by the shearing deformation of two-dimensional bubble glasses [189], Argon proposed that the number of atoms involved the "flow event" should be a few to $\sim 100$ [190]. The "flow event" can be described by a local plastic or inelastic rearrangement of atom clusters, commonly termed as the "shear-transformation zone" (STZ). The first quantitative model of the STZ behavior was developed by Argon himself, who treated the problem in the context of an Eshelby-type inclusion [191, 192]. He argued that an isolated STZ is not free, but confined to the surrounding elastic matrix. Using Eshelby's insightful theory, Argon calculated the free energy for the STZ activation in the SYZ-elastic matrix system as

$$
\Delta F_{0}=\left[\frac{7-5 v}{30(1-v)}+\frac{2(1+v)}{9(1-v)} \ell^{2}+\frac{1}{2 \gamma_{s t z}} \frac{\tau_{0}}{G(T)}\right] G(T) \gamma_{c}^{2} \Omega_{c}
$$

where $\tau_{0}$ is the athermal shear stress at which the STZ transforms, and $G(T)$ is the temperature-dependent shear modulus. The second term in the bracket captures the dilatational energy associated with the STZ operation, and $\ell$ is the ratio of the dilatation to the shear strain, called a dilatation factor. The characteristic strain, $\gamma_{c}$, of an STZ with the characteristic volume, $\Omega_{c}$, depends on glass compositions and structural states, being of order $\sim 0.1$ at high temperatures and $\sim 1.0$ at low temperatures. Under steady-state conditions, the STZ operations can occur in favor of and in opposition to the sign of the applied shear stress. Each net forward operation ascribes, $\gamma_{c}$, to the steady-state macroscopic shear strain, $\dot{\gamma}$, which is given by

$$
\dot{\gamma}=C_{\xi} \gamma_{c} H_{\mathrm{STZ}}
$$


where $C_{\xi}$ is the concentration of STZs, namely the fraction of a material that is available to STZ operations in a unit volume element, and the net activation frequency, $H_{\mathrm{STZ}}$, of an STZ obeys a rate law of the form:

$$
H_{\mathrm{STZ}}=f\left[\exp \left(-\frac{Q-\tau \Omega_{c}}{k_{B} T}\right)-\exp \left(-\frac{Q+\tau \Omega_{c}}{k_{B} T}\right)\right]
$$

Here, $Q$ is the activation-energy barrier for an STZ under an unstressed field. It is important to point out that an STZ is not a structural defect in metallic glasses, as Argon suggested [190]. The STZ is essentially a local cluster of atoms that undergoes an inelastic shear distortion from one relatively-low-energy configuration to a second such configuration, crossing an activated configuration of higher energies and volumes. The transitions between STZ states could be thermally activated. In a manner, an STZ in metallic glasses has an analogy with a dislocation motion in crystals, but on short-range order (SRO) length scales. Although an STZ is a "flow event", not a defect, it is strongly affected by local atomic structures, such as free volumes, short-range chemical or topological order. For example, the availability of free volumes is important for STZs to operate in a given volume of BMGs. In fact, STZ operations occur preferentially in those regions having a higher free volume, as relatively less dilatation is required. Later, some people [193-195] introduced a concept of the "flow defect" analogous to STZs in terms of free volumes. He succeeded in extending individual atomic jumps to atomic-cluster rearrangements and derived an expression of strain rates quite similar to Eq. (10). Recently, much work [196, 197] has successfully developed the STZ dynamics by taking the free volume into account. 
Since Argon proposed the STZ model, scientists have made great efforts to capture such a "flow event" via experimental or simulation ways. About 20 years later, Falk and Langer [198] for the first time observed the STZ-type atomic motion by atomistically-modeling viscoplastic deformation in amorphous solids. STZs can be treated as pre-existing "flow defects" in metallic glasses, which comprise a group of atoms susceptible to structural rearrangements upon external agitations. Based on the microscopic observations, they argued that the density and internal states of these STZs should be added to the constitutive description of metallic glasses. At the temperatures well below the glass temperature, the transitions between STZ states are not thermally activated but, rather, are controlled entropically. That is, the rate factors are determined by the number of paths that the molecules within a zone can follow in moving around each other while going from one state to the other [198]. Considering the two-state nature of STZs (denoted by the symbols"+" and "-"), the time-dependent number densities, $n_{ \pm}$, of STZs in those states can be written by

$$
\dot{n}_{ \pm}=R_{\mp} n_{\mp}-R_{ \pm} n_{ \pm}-C_{1}(\tau \dot{\gamma}) n_{ \pm}+C_{2}(\tau \dot{\gamma})
$$

where $R_{ \pm}$are the rates at which \pm states transform to $\mp$ states that are the free volume (or entropy) activated at temperatures well below the glass-transition temperature, $C_{1}$ and $C_{2}$ are coefficients related to the annihilation and creation of STZs, respectively. The plastic strain rate, $\dot{\gamma}^{p}$, has the form

$$
\dot{\gamma}^{p}=\Omega_{c} \Delta \gamma\left[R_{+} n_{+}-R_{-} n_{-}\right]
$$


where $\Delta \gamma$ is the increment of the local shear strain. These results provide the richness to the STZ formulism. Experimentally, Schall et al. [199] used a colloidal glass and identified localized irreversible STZs in the presence of an applied shear.

\subsection{Tension-transformation-zone (TTZ) model}

In metallic glasses, the distortional (shear) and volumetric (dilatation) components of deformation are highly coupled [179-181, 200, 201], which distinguishes from that of their crystalline counterparts. The inherent competition between shear and dilatation deformations determines the materials' plasticity, which is indicated by the well-known Poisson's ratio criterion for plasticity, i.e., the larger the Poisson's ratio is, the more plastic of BMGs [202-204]. As for the distortional component of deformation, which is well described by the STZ model [190]. But we should keep in mind that although it is shear dominated, the STZ is always accompanied by a slight dilatation (or free-volume creation) [195, 205]. In most cases, the STZ-type deformation is predominated, resulting in the macroscopic (homogeneous or inhomogeneous) plastic flow in metallic glasses. Nevertheless, a question naturally arises: whether the dilatation could dominate the deformation process of metallic glasses or not? If the answer is affirmative, what does that mean?

An intriguing work reported by Xi et al. [206] opens a window into the answer to the above questions. They observed that in some brittle metallic glasses, the apparent mirror-like zone on the facture surface exhibits the unique, nanoscale periodic corrugation (NPC). The further investigation reveals that the NPCs are peak-to-peak or 
valley-to-valley matched [207]. This tread implies that the dilatation (or cavitation) should dominate the deformation process of materials in front of the crack tip. In a typical tough metallic glass (Vitreloy 1), Jiang et al. [208, 209] also observed the NPC on the dynamic mode-I fracture surface, indicating the universality of NPCs. They used high-resolution atomic-force microscopy (AFM) to scan the NPC, and found that NPCs are actually a series of self-organized nanovoids, as shown in Figure 27(a) [210]. The nucleation sites of nanovoids construct the valley part of the NPC, while the link sites of nanovoids correspond to the peak part. The repeated nucleation and link of these nanovoids lead to the formation of the NPC, as illustrated in Figure 27(b) [209]. Jiang et al. [210] further adopted a detrended fluctuation analysis to quantitatively analyze the AFM profiles of the NPC. They revealed that the landscape of the valley part of the NPC are almost memoryless with a significant fractal feature. This feature means that the formation of these nanovoids is quasi-brittle with very limited plastic growth.

On the atomistic scale, Jiang et al. [209] proposed a basic, atomic cluster motion, defined as a tension transformation zone (TTZ), to describe the quasi-brittle dilatation deformation in metallic glasses. Conceptually, a TTZ can be regarded as the counterpart of a STZ suffering a remarkable dilatation but only a slight shearing. TTZs are less viscoplastic than STZs and more prone to the fracture than flow, when subjected to stresses. Through TTZs, the stored energy is dissipated mainly by the new surface formation, resulting in the brittle fracture of metallic glasses. Conversely, the STZ-mediated deformation always lead to the ductile fracture of metallic glasses where the significant plastic flow occurs ahead of the crack tip [211-213]. Therefore, the 
ductile-to-brittle transition (DBT) of metallic glasses can be well understood within the picture of STZ versus TTZ of atomic-cluster motions [214-216], as illustrated in Figure 28 [217]. Jiang et al. [218] have performed a series of three-point-bending experiments on as-cast and annealed Vitreloy 105 metallic glasses over a wide range of temperatures, where a clear DBT was observed. By connecting the macroscopic DBT and the microscopic STZ-to-TTZ transition, they predicted a critical size (a TTZ volume) of cluster motions corresponding to the transition. According to the TTZ concept, the characteristic spacing of NPCs can be predicted [209, 219], which agrees well with the experimental measurements.

The TTZ-type motion of atomic clusters has been confirmed by a recent work reported by Murali et al [220]. They performed atomistic simulations on the fracture behavior of two typical metallic glasses, one brittle $(\mathrm{FeP})$ and the other ductile $(\mathrm{CuZr})$. The results shows that brittle fracture occurs in the FeP glass where the crack tip propagates by a series of the nanoscaled void nucleation and coalescence processes with the very limited plastic growth, while blunting of the crack tip through extensive shear banding, indicative of a ductile fracture, is seen in the CuZr glass. The nanoscaled void nucleation is essentially the TTZ model proposed by Jiang et al. [209]. Obviously, TTZ shows some differences, compared to the classical cavitation process. Firstly, TTZ is at the nucleation stage of cavitation, during which new surface formation is the main way to dissipate the applied energy, and plastic growth can be neglected. Second, TTZ occurs usually on a nanoscale or even sub-nanoscale, while the classical cavitation is usually at a length scale more than the micrometer. Finally, the TTZ can be activated by 
the shear-induced dilatation or direct hydrostatic tension, whereas the classical cavitation is activated mainly by the direct hydrostatic tension. Like STZ, TTZ is also not a structural defect, but a dynamic "event". Therefore, the TTZ model still needs further experimental evidence.

\subsection{Cooperative shear model}

Even though the free-volume, STZ, and TTZ models provide explanations for the strain softening and the heterogeneous deformation in metallic glasses, it is difficult to quantitatively describe the strength and ductility of metallic glasses at room temperature. Based on the concept of inherent states (IS) and potential-energy landscapes (PEL) developed by Stillinger et al. [221, 222], Doye and Wales [223, 224], and Milandro and Lacks [225], Johnson et al. [226] proposed a cooperative shear model (CSM) to characterize the deformation in metallic glasses. Later, Demetriou et al. [227] applied the CSM for the rheology of the glass-forming metallic liquid. A quantitative equation is given as follows:

$$
\alpha \eta \dot{\gamma}^{2} \delta W / \delta \varepsilon=\frac{\left(W-W_{e}\right)\left(W / W_{0}\right)^{q}}{\eta / G_{0}}
$$

where $\dot{\gamma}$ is the strain rate, and $\alpha$ is a parameter incorporating two unknown proportionality constants: the conversion efficiency of the dissipated energy into the potential energy and a factor quantifying the deviation of the system's relaxation rate from Maxwellian, $\eta$ is the viscosity, $W$ is the activation barrier for the shear flow, which involves the shear modulus given by the curvature of the energy-density function, and $\varepsilon$ is the flow-induced shift in the specific configurational potential energy of shear zones 
[227]. Pan et al. [228] have proposed an experimental scheme to characterize STZs of plastic flows in BMGs, and obtained the measured STZ volumes in different glass-forming systems, which are determined to be $2.54 \sim 6.56 \mathrm{~nm}^{3}$. The accordance between the measured STZ volumes and those predicted by theoretical analyses [226] and molecular-dynamic (MD) simulations [229] unambiguously demonstrates that the plastic deformation of metallic glasses occurs upon cooperative shearing of unstable clusters of atoms instead of the individual atom motion. STZs with a large size reinforce the shear capability of the metallic glass and promote the formation of multiple shear bands, which concurs with the fact that a higher Poisson's ratio is available [228].

\subsection{Flow-unit model}

Very recently, to understand the plasticity and glass transition in metallic glasses (MGs), Wang et al. [230-235] proposed the dynamic flow-unit model, according to which, MGs can be regarded as the composite of the perfect elastic matrix and liquid like flow units ( $\mathrm{MG}=$ ideal glass + flow units), and the flow phenomenon in MGs is the percolation of the localized flow units. Jiao et al. [230] present the experimental results on the distribution and evolution of energy barriers of flow units in MGs via an activation-relaxation method. The dynamical heterogeneity of MGs arises from its structural inhomogeneity, and there exist the close correlations among the flow units, dynamical and structural heterogeneities, and relaxation behaviors in MGs. Zhu et al. [232] experimentally characterized the evolution of flow units associated with the 
flow "defects" in MGs by monitoring the fictive temperature change of a typical metallic glass.

Up to now, the $\beta$ relaxation has been considered to be the dynamic response of the atoms in the flow units, which can be detected by the dynamic spectrum like dynamic mechanical spectroscopy [230-238]. The $\beta$ relaxation has the comparable activation energy with that of the flow unit and is closely related to the initiation and evolution of the flow units in MGs. As a result, the flow of MGs is correlated with inherent relaxation processes. Thus, the distribution and evolution of energy barriers, size, and intrinsic relaxation time of flow units in a MG can be determined through the $\beta$-relaxation studies using an activation-relaxation method [238]. The pronounced macroscopic tensile plasticity is achieved in a La-based MG, which possesses strong $\beta$ relaxations and nanoscale heterogeneous structures [233].

Based on Ref. [235], a comprehensive picture on the hidden flow as well as its correlation with deformation maps and relaxation spectrum is proposed. As exhibited in Figure 29 [235], below the $\beta$-relaxation peak, $T_{\mathrm{T} 1}$, only reversible flow units can be activated, as illustrated by the high mobility atoms (red balls) embedded in the elastic shell (blue balls). The flow units with high-concentration free volumes may be related with the high-density inhomogeneity. Such isolated reversible motions within the matrix can be regarded as hopping events across inherent structures and contribute to the $\beta$ relaxation. The brittle nature of MGs is not changed by these few easy flowing spots. With increasing the temperature, adjacent weak-bonded regions around flow-unit regions are gradually transformed into a liquid state, since the applied 
strain lowers the energy barrier and increases the fraction of the flow units. At this stage, the liquid-like zones lead to localized plastic events and lower the yielding stress, but the MGs do not exhibit macroscopic plasticity.

At around the $\beta$-relaxation-peak temperature, $T_{\beta}$, a connectivity percolation of liquid-like zones takes place. The percolation of the flow units facilitates the multiplication of shear bands. For most MGs, $T_{\beta}$ is located at about $0.8-0.9 T_{\mathrm{g}}$. Consequently, homogeneous deformation easily happens. Usually, the sub- $T_{\mathrm{g}}$ endothermic peak is observed, which is thought to be related with the $\beta$ relaxation. The cooperative translational movements take place above $T_{\beta}$ and can be regarded as a kind of confined glass-to-liquid transition (GLT), leading to an endothermic peak. Such hidden flowing movements need external energy to facilitate.

When the temperature approaches $T_{\mathrm{g}}$, the sample behaves like a macroscopic flow, associated with the distinguished plasticity. A heat capacity jump of about $3 \mathrm{R} / 2$ related to the translational freedom addition can be observed and a critical transition from the broken-ergodic to ergodic states on the energy landscape occurs. The sample transforms into a liquid-like state above $T_{\mathrm{g}}$, the viscosity change becomes the dominating factor for the deformation behavior. As viscosity rapidly decreases and approaches the $\alpha$-relaxation peak, $T_{\alpha}$, the sample transitions from the non-Newtonian to Newtonian flows.

\subsection{Serration kinetics}


In crystalline alloys, the motion of dislocations is related to the macroscopic-plastic deformation. An intriguing example of the plastic deformation is characterized by the Portevin-Le Chârtelier (PLC) effect [239], described as the repetitive discontinuous yielding in the stress-strain curve subjected to a constant strain rate. A typical Al alloy system exhibits the PLC effect [240, 241], in which the inhomogeneous deformation with various localization bands caused by the PLC effect is observed, and the onset of the jerk flow is commonly attributed to a strain-rate softening instability, resulting from the dynamic-strain-ageing (DSA) mechanism [242]. A serrated flow, similar to the PLC effect known for crystalline alloys, is a characteristic feature of the inhomogeneous plastic deformation of BMGs on stress-strain curves [243-251]. It has been demonstrated that the serrations are highly related to shear banding during deformation in metallic glasses [247, 248]. Theoretical explanations on shear banding could unveil the underlying physical origins in metallic glasses.

Jiang et al. [252] have found that one serration event could correspond to the operation of several shear bands simultaneously. Once yielding, serrated flows in stress-strain curves dominate. In the stress-ascending stage, BMGs are under straining. On the other hand, in the stress-dropping stage, a rapid decrease of stresses results in the formation of a viscous shearing layer, accompanied by the increase of free volumes. The strain is accommodated within the shearing layer, i.e., the straining energy, produced in the stress-ascending stage, is dissipated. Repeatedly, as stresses are 
increasing during compression, strains are accommodated elastically, until the stress level reaches the value that it can activate a next serrated flow.

Antonaglia et al. [251] employed the mean-field interaction model to predict the scaling behavior of the distribution, $D\left(S^{*}\right)$, of avalanche sizes, $S^{*}$, in the experiments. $D\left(S^{*}\right)$ follows a power law multiplied by an exponentially-decaying scaling function. The size of the largest observed avalanche depends on the experimental tuning parameters, such as either the imposed strain rate or stress. Similar to crystalline materials, the plasticity of BMGs reflects the tuned criticality showing a remarkable quantitative agreement with the slip statistics of slowly-compressed nanocrystals.

Sun et al. [244, 249] and Wang et al. [253] have recently reported a close correlation between the dynamic behavior of the serrated flow and the plasticity in metallic glasses and show that the plastic deformation of ductile metallic glasses can evolve into a self-organized critical (SOC) state characterized by the power-law distribution of shear avalanches. The stick-slip model is most popular in depicting the serration kinetics [244, 249, 253-257], as follows:

The machine-sample system (MSS) is loaded at a low constant rate, $v$, from the time, $t=0$, as presented in Figure 30 . The elastic energy is gradually stored in the system, providing the driving force, $k_{M} k_{S} v t /\left(k_{M}+k_{S}\right)$, with $k_{M}$ and $k_{S}$ being the stiffnesses of the machine and the sample, respectively. Once the sample is loaded up to the yielding stress, the primary shear bands will generate, the elastic energy stored in the MSS will be released, and the shear bands will quickly slip across the entire sample in a cooperative manner. The governing kinetic equation for MSS is expressed as: 


$$
\frac{k_{M} k_{S}}{k_{M}+k_{S}}(v t-x)-\frac{\pi d^{2}}{4} \sigma_{b}=M x^{\prime \prime}
$$

where $x$ is the vertical plastic shear displacement, $d$ is the sample diameter, $M$ is the effective inertia of MSS, typically on the order of $10-100 \mathrm{~kg}$ [249], and $x$ " is the second derivative of $x . \sigma_{b}$ is the internal resistant stress of the shear band, which can be given by the constitutive law. Defining the elastic constant:

$$
k=\frac{4 k_{M} k_{S}}{\pi d^{2}\left(k_{M}+k_{S}\right)}=\frac{E}{L(1+S)}
$$

where $L$ and $E$ are the height and Young's modulus of the sample, respectively, and $S$ is defined by $S=k_{S} / k_{M}=\pi d^{2} E /\left(4 L k_{M}\right)$. Eq. (15) becomes:

$$
k(v t-x)-\sigma_{b}=p x^{\prime \prime}
$$

with $p=4 M / \pi d^{2}$. From Eq. (17), $k$ and $\sigma_{b}$ can be treated as the factors of shear banding, the stiffness of the system, and the intrinsic properties of BMGs. Only the single shear-band movement is discussed in this model. Profuse shear bands are mutually operated. It is assumed that each shear band is a single unit. The driving force is $k_{M} k_{S} \sum x_{j} /\left(k_{M}+n k_{S}\right)$, here, $n$ is the number of units, $x_{j}$ is the displacement of the $j$ th shear band, and $\sum x_{j}$ is the sum over all units. With the same treatment, the kinetic equation for the system is:

$$
k^{\prime}\left(v t-x_{i}\right)+k^{\prime}\left(x_{i}-\frac{1}{n} \sum x_{j}\right)-\sigma_{b i}=p^{\prime} x^{\prime \prime}, i=1,2 \ldots n
$$

where $\sigma_{b i}$ is the internal resistance of the $i$ th shear band, $p^{\prime}=4 M / \pi d^{2}$, $k^{\prime}=\frac{4 n k_{M} k_{S}}{\pi d^{2}\left(k_{M}+n k_{S}\right)}=\frac{E}{L(1 / n+S)}$, and $k^{\prime}\left(x_{i}-\frac{1}{n} \sum x_{j}\right)$ is the interactions among shear bands. It reflects the effect of other shear-band movements during sliding on the $i$ th shear band. The shear-band interactions are perfectly described by the MSS model, 
which will significantly affect the serration dynamics. It is found that the intermittent sliding of shear bands will cause serrations on the stress-strain curves. Sun et al. [249, 254] have found that when the spring stiffness was lower than the critical value, the shear-band slipping would become unstable, and the stick-slip motion appeared. Based on this criterion, the transition from the serrated to nonserrated flows with the strain rate or temperature is well predicted, and the effects of various extrinsic and intrinsic factors on the shear-band stability can be quantitatively analyzed in BMGs. Han et al. [258] developed a model to explain the influence of the stiffness of the machine and sample achieved in the device on the plastic deformation of BMGs. It is found that the larger the critical $S$ is, the easier the stable behavior can be achieved. As the aspect ratio (length / diameter) is fixed, the smaller the sample is, the stronger effect that the energy stored in the testing machine has on the mechanical behavior of BMGs, e.g., the larger serration magnitude is obtained for the smaller sample.

Recent progresses in serration kinetics strongly correlate the serration in metallic glasses with shear banding, affected by the structure of shear bands [259], the temperature rise of shear bands during deformation [260], the compositional dependence of the shear-band dynamics [261], and the velocity of propagating shear bands [245, 248], and features, which are all indispensable for a full description and understanding of shear-band dynamics. More comprehensive mechanisms are expected. For example, very recently, we have unveiled an exponential decay of shearing stresses with the time or strain during serrations in BMGs [253, 262]. The flow stresses with the time satisfy the following relation: $\sigma=A \cdot e^{-B \cdot x}[253,262]$. The burst sizes, $\Delta \sigma$, in different BMGs with varied dimensions are predicted by $\Delta \sigma=\sqrt{\frac{2 l_{v} E \rho c_{p}\left(0.8 T_{g}-T_{r}\right)}{0.9 a D \sin \theta}}[263]$. Here, $D$ is the diameter, $l_{\mathrm{v}}$ is the thickness of the 
shearing layer, $\rho$ is the density, $c_{p}$ is the heat capacity, $T_{\mathrm{r}}$ is room temperature, $T_{\mathrm{g}}$ is the glass-transition temperature, $E$ is the Young's modulus of BMGs, and $a$ is the aspect ratio of the tested samples. In a word, affected by all kinds of intrinsic and extrinsic factors $[264,265]$, understanding the plasticity is still a great challenge.

\subsection{Universal equation for the strength in metallic glasses}

In the absence of defects, such as dislocations or grain boundaries, the fracture strength of metallic glasses is considered to be close to the theoretical strength of ideal solids [266]. Different BMGs exhibit large variations of fracture strengths, e.g., Ce-based BMGs with a fracture strength of $\sim 500 \mathrm{MPa}$ at room temperature [267], but the Co-based BMGs with $\sim 5,000 \mathrm{MPa}$, one order larger than the former [268]. The understanding of the material parameters governing the strength of the amorphous structure is, therefore, of essential importance to the future design of BMGs with desired mechanical properties.

The fracture strength of BMGs is believed to be directly associated with the atomic bond due to the lack of defects, such as dislocations [5]. Thus, the strength of a $\mathrm{BMG}$ is expected to be closely related to the physical parameters determined by the atomic-cohesive energy. Whereas the thermal energy for the glass transition spreads around the entire body of the solids during heating, the mechanical energy of shearing is highly confined in the localized shear bands of BMGs. The energy density, a variable that only depends on the initial and final states, can be expected to be similar for the thermal and mechanical processes [269]. This investigation gives:

$$
\tau_{y} \gamma_{0} \approx \int_{T_{0}}^{T_{g}} \rho C_{p} d T
$$


where $\tau_{y}$ is the maximum shear stress upon yielding, $\gamma_{0}$ is the shear strain of the basic shear unit (STZ), $\rho$ is the density of the material, $C_{\mathrm{p}}$ is the heat capacity, $T_{\mathrm{g}}$ is the glass-transition temperature, and $T_{0}$ is the ambient temperature. From Eq. (19), it is assumed that once the temperature within the shearing layer reaches $T_{\mathrm{g}}$, a viscous shearing layer forms, which could not withstand the propagation of shear bands, and softening happens, accompanied by the catastrophic failure. Usually, the yielding strength, $\sigma_{y}=2 \tau_{y}$, since the shearing angle is approximate 45 degree, with respect to the loading direction. After a series of simplifications, all the variables are determined, according to the classical thermodynamics. This process gives:

$$
\sigma_{y}=50 \frac{T_{g}-T_{0}}{V}=50 \frac{\Delta T_{g}}{V}
$$

where $V$ is the molar volume of BMGs. Generally, the final fracture strength is slightly larger than the yielding strength, and an average ratio, $\sigma_{f} / \sigma_{y}=1.1$ is adopted:

$$
\sigma_{f}=1.1 \sigma_{y}=55 \frac{\Delta T_{g}}{V}=55 \frac{\rho_{0}}{M} \Delta T_{g}
$$

where $\rho_{0}$ is the density at room temperature, and $M$ is the molar mass. The calculated fracture strength from Eq. (21) versus the measured values of 27 BMGs from 11 different BMG systems is shown in Figure 31 [269]. A remarkable agreement is observed between the calculated and measured strength values. This study provides a physical understanding for the strength of BMGs, based on their unique deformation mode of localized shear banding. In particular, a simple unified equation is derived to allow the calculation of the strength of a BMG directly from their $T_{\mathrm{g}}$ and other material constants that can be physically determined. Furthermore, $T_{\mathrm{g}}$ of a variety of MGs shows 
a heredity from the eutectic or peritectic point, $T_{\mathrm{m}(\mathrm{AxBy})}$, which is from the binary phase diagram of two base elements in MGs [270]. $T_{g}=0.55 T_{m(A x B y)}$ is universally identified in many different glass-forming systems [270]. Here, $T_{\mathrm{m}(\mathrm{AxBy})}$ is highly dependent on the melting points of two dominant base components. As a result, a more universal equation for the fracture strength, $\sigma_{f}$, can be predicted by the following equation:

$$
\sigma_{f}=0.01 \frac{\rho_{0}}{M} T_{m(A x B y)}-55 \frac{\rho_{0}}{M} T_{0}
$$

\subsection{Introduction of the simulation work}

Computational modeling is an effective means in the understanding of materials' structures and properties, including physical and mechanical behavior. Up to now, many research efforts have been conducted to incorporate theory and modeling to complement experimental investigations, which play several key roles in research, particularly in disordered systems. For example, the simulations of liquids and glasses can date back to the earliest stages of simulation studies [271, 272]. The simulation work can provide key tests of theory, giving unparalleled insights that lead to new theoretical ideas, and providing a connection between theory and experiments.

Despite the long history of BMGs, many outstanding fundamental scientific issues remain unclear, because there are no appropriate experimental techniques to detect localized stress distributions and localized structural changes in amorphous structures, such as: (1) Is the yield stress the localized-shear deformation initiation-controlled or propagation-controlled? (2) Does the propagation of shear bands occur purely mechanically or accompanied by a thermal activation? (3) Why the widths of shear bands are kept so narrow $(10-20 \mathrm{~nm}$ [36]) even after severe 
deformation? (4) Whether or not the shear-band propagation can be described as the dislocation propagation? and (5) Why the yield strain is insensitive to alloy systems?

From the computational point of view, a major advantage of simulations on studying the structures and deformation mechanisms of BMGs is that a detailed picture of the model under investigation is available, and, therefore, they have been very helpful in explaining the connection between macroscopic properties and atomic structures. For example, atomistic simulations can be employed to investigate the following scientific issues [273]: (1) the construction of a model structure, (2) the deformation simulation, (3) analyses of the structures and properties of deformation sites and their changes with plastic deformation, and (4) the extraction of some physical concepts concerning deformation mechanisms. Among all the above studies, the atomistic simulation of the shear-band formation is of great significance with multiple reasons: (1) the atomistic simulation is one of the only few available methods to approach the atomistic process of the shear-band formation; (2) the mechanism of the shear-band formation seems to be common to any metallic glass, meaning that the phenomenon is not sensitive to the details of interatomic potentials; and (3) the thickness of the shear bands is as narrow as $10-20 \mathrm{~nm}$, the size of which can be accessible by the present large-scale computers. In this review article, we will focus on the formation mechanism of shear bands in metallic-glass-matrix composites.

Simulations can also be used to compare with experimental results to validate the model. Thus, one can use simulation results to measure properties that are not accessible to experiments. In many cases, as will be demonstrated in the next sections, 
simulation results can motivate experimental investigations. However, it should be noted that while simulations have provided significant insights, they also face many limitations. Just as experimental techniques, such as microscopy, calorimetry, and diffraction, are critical for understanding materials by providing the important information, the simulation methods also have inherent limitations, including limited time and length scales. 


\section{Deformation of metallic glass matrix composites under uniaxial quasi-static loading}

\subsection{Deformation of BMGs under quasi-static compression and quasi-static tension at room temperature}

\subsubsection{Compression of BMGs}

Almost all the monolithic BMGs exhibit very limited compressive plasticity, usually less than $2 \%$ at room temperature [274]. The brittle behavior is analogous to the one in the ceramics. When people know how to design more free volumes in the samples, certain plasticity is obtained, e.g., most samples for compression are selected to be less than $2 \mathrm{~mm}$ in diameter. Even so, the intrinsic brittle nature has not been alleviated so far. After yielding, a number of serrations, associated with the accommodation of plasticity, appear on the stress-strain curves [247, 248, 251, 252]. The more serrations, the more plastic the BMGs is. The occurrence of serrations can be explained by the multi-step shearing models [275].

Taking the shear bands as a source of zero thickness in an infinite medium, a detailed temperature-rise $(\Delta T)$ profile along the width direction of shear bands, $x$, and with the time, $t$, can be performed by solving the heat-diffusion equation:

$$
\Delta T=\left(\frac{H}{2 \rho C_{p} \sqrt{\pi \alpha^{\prime}}}\right) \frac{1}{\sqrt{t}} \exp \left(\frac{-x^{2}}{4 \alpha^{\prime} t}\right)
$$

where $H$ is the energy per unit shear surface, $C_{p}$ is the heat capacity, and $\alpha^{\prime}$ is the thermal diffusivity, and $\rho$ is the density. Generally, the measured shear offset is a result of multi-step shearing [275], and each serration contributes to the finally-observed shear offset. After calculations, the thermal energy converted from the storage elastic 
energy during serrations is not enough to result in $\Delta T$ higher than $T_{\mathrm{m}}$, even though there are few $\Delta T$ values higher than $0.8 T_{\mathrm{g}}$. Only if the final fracture is available, $\Delta T$ is greatly higher than $T_{\mathrm{m}}$, which causes that the shear layer could not sustain the loading [275]. Consequently, stable compressive plastic flows happen.

Making a strong yet stretchable glass poses a great challenge to materials scientists. During the past years, people have been always looking for ways to improve the room-temperature plasticity of monolithic BMGs on the premise of keeping the amorphous structure. By the simple method of shot-peening [276], BMGs show increased plasticity in bending and in compression, through a combination of the reduced likelihood of surface cracking and more uniform deformation induced by a high population of pre-existing shear bands. By the introduction of the geometrical constraints $[252,277-280]$, the outstanding plasticity is naturally obtained. The intrinsically-improved plasticity has been achieved by designing BMGs with high Possion's ratios [84, 202], and super plasticity emerges, as found in $\mathrm{Zr}-\mathrm{Al}-\mathrm{Ni}-\mathrm{Cu}$ systems [84]. Like pursuing the high GFA, it is of importance to render BMGs with superior plasticity without strength degradation.

\subsubsection{Tension of BMGs}

The strength-ductility paradox in BMGs under tension is a long lasting problem, which is yet to be tackled for structural applications despite that substantial efforts have been paid over the past decades. In bulk samples with the thickness or diameter larger than $1 \mathrm{~mm}$, there has no tensile ductility found unless they are through the artificial design. For example, it is found that a complex stress distribution in 
notched BMGs resulted in a distinguished tensile ductility [281, 282]. By the carefully-controlled surface mechanical attrition treatment, the formation of gradient amorphous microstructures across the sample thickness effectively promote multiple shear banding, while delaying the cavitation in BMGs, thus resulting in superior tensile ductility [283]. However, there are numerous reports on finding the tensile ductility in micro- even nano-scale metallic glasses, such as films or wires [284-289]. An outstanding example is that the $\mathrm{Al}_{90} \mathrm{Fe}_{5} \mathrm{Ce}_{5}$ metallic glass with a size $<20 \mathrm{~nm}$ can be extremely elongated to $\sim 200 \%$ [286]. In such a small dimension, STZs will cooperate strongly with each other, and propagate throughout the whole sample. Meanwhile, the short-range or modest-range strain relaxation via atom diffusion will be considerable, which also assists the homogeneous deformation $[285,286]$.

The asymmetry of compression and tension in BMGs has been revealed [179]. Under tension, free propagation of shear bands facilitates crack opening. Only if structural heterogeneity exists, the free propagation could be inhibited. In comparison, crack evolution from shear bands is retarded even if shear banding occurs, since the normal pressure is available under compression, which goes against crack opening. This is why very limited compressive plasticity is popular, while zero tensile ductility prevails in BMGs. The notification of such asymmetry reminds us of importing structural heterogeneity in BMGs so as to improve the plasticity and toughness.

\subsubsection{Size-dependent mechanical properties}

Due to that metals and alloys are most common in their polycrystalline form, the interaction between defects and grain boundaries is particularly interesting. In 
bulk, grain boundaries act as obstacles to dislocation motion as conveyed through the classical Hall-Petch relation, describing the characteristic increase in the yielding strength of polycrystalline metals with decreasing the obstacle distance, down to the grain sizes of about $40 \mathrm{~nm}$ [290]. However, when the grains are reduced to about 40 $\mathrm{nm}$, the polycrystalline metals cannot accommodate multiple dislocations, which engages alternative plastic-deformation mechanisms like grain-boundary sliding, partial dislocation emission, and absorption at grain boundaries [291]. At grain sizes below $20 \mathrm{~nm}$, the Hall-Petch relation gives way to the so-called “inverse", Hall-Petch, manifested through softening with the decreased grain size due to the activation of the grain boundary-assisted deformation.

But for amorphous alloys without dislocations and grain boundaries, although the size-dependent mechanical response is existing, the deformation mechanisms are totally different from those in crystalline alloys. As discussed above, most of BMGs exhibit zero tensile ductility and little compressive plasticity at room temperature. In comparison, when the diameter or thickness of MGs is reduced to micro- and / or nano-scale, the pronounced tensile ductility and compressive plasticity are available, accompanied by serrations on the stress-strain curves after yielding [284-289, 291-293]. Upon decreasing the diameters of amorphous alloys, the deformation shows more pronounced intermittent characteristics, which is due either to the more frequent initiation of new shear bands or to repeated post-initiation growth (reactivation) of pre-existing shear bands in multiple steps. 
From the values of the yielding strength measured from a large number of Cu-based and Zr-based micropillars [284, 294], there is no dependence of the yielding stress on the pillar diameter over the current size scale, since the strength of amorphous alloys is essentially controlled by the interatomic bonding and has a roughly linear relationship with the elastic modulus [226]. In other words, "smaller is stronger"' is not present in amorphous alloys, as observed in nano-sized crystalline alloys due to dislocation nucleation rather than multiplication controlled mechanisms.

As far as the size-dependent plasticity is concerned, the tensile ductility and compressive plasticity are easily obtained in micro- and nano-scaled amorphous alloys even if in very brittle Fe- and Mg-based alloys [292, 295]. Greer et al. [291] gave some explanations for the size-dependent plasticity in amorphous alloys, as discussed below. Due to larger stress drops in smaller diameter pillars, a shear band tends to stop. Consequently, it requires the re-building of the stress to re-activate the pre-existed shear bands or to initiate another one. In a thick pillar, a propagating shear band has a less opportunity to stop due to small load drops. Specially in BMGs, the nucleation of a shear band may directly lead to the catastrophic failure. In this sense, the deformation of larger specimens is essentially controlled by the nucleation of shear bands. With decreasing the sample diameter, however, the frequent arrest of shear bands indicates that the deformation becomes more likely controlled by shear-band propagation. Therefore, more serrations dominate with decreasing the sizes, which is related to larger plasticity. And the improved plasticity is easily obtained.

\subsection{Deformation of MGMCs under quasi-static compression}




\subsubsection{Compression at room temperature}

Since most engineering materials serve at room temperature, it is popular to study the room-temperature mechanical behaviors. As stated above, the introduction of secondary crystalline phases in BMGs as the structural heterogeneity effectively improves the room-temperature toughness. The secondary phases themselves act as carriers to absorb the plasticity. And profuse shear bands induced by the blockage of shear-band propagation can accommodate more plasticity $[95,101]$.

\section{1) Compression of ex-situ composites}

As far as ex-situ MGMCs are concerned, especially for intermetallics particle-reinforced composites, the improvement of plasticity mainly origins from resultant multiple shear bands, while the carrier absorbability by particles themselves is little, as the dislocation proliferation in hard and brittle intermatallics is very difficult. On contraries, in ductile-metal particles or metal-fiber reinforced MGMCs, both shear bands and metal carriers contribute to the macroscopic plasticity.

Here, we first give a brief statement on the long fiber / metallic glass composites. MGMCs reinforced with $110-\mu \mathrm{m}$ and $250-\mu \mathrm{m}$-diameter STS304 stainless steel fibers are referred to as 'S1' and 'S2', respectively, and the LM1 BMG is referred to as Vit1 [66]. The SEM image of the S2 composite is shown in Figure 32(a) [66]. The STS fibers are randomly distributed in the matrix, and their volume fraction is about 65 and $68 \%$ in the S1 and S2 composites, respectively. Figure 32(b) shows typical engineering compressive stress-strain curves of the LM1 alloy and S1 and S2 composites [66]. The yielding and maximum strengths of the LM1 alloy are 1,664 
$\mathrm{MPa}$, and the plastic strain is hardly observed. In the S1 composite, the maximum strength is $1,062 \mathrm{MPa}$, and the fracture does not take place even above the strain of 32 $\%$. The maximum strength of the $\mathrm{S} 2$ composite is $1,204 \mathrm{MPa}$, which is slightly higher than that of the $\mathrm{S} 1$ composite, but the fracture starts at the plastic strain of $11 \%$.

Overall, the long fiber-reinforced MGMCs exhibit large compressive plasticity under quasi-static loading at room temperature $[56,59,60,66,296]$.

The deformation mechanisms of fiber-reinforced MGMCs are mainly from Ref. [296]. After carefully checking the fracture morphologies, it is found that the failure mode of the tungsten composite may change from the shear fracture to splitting fracture with increasing the fiber volume fraction [296]. Shear and splitting fracture mechanisms at room temperature are discussed as below [296]. Usually, the shear-fracture angle under compression is less than $45^{\circ}$. Thus, the Mohr-Coulomb criterion has been used to explain the deviation from the maximum shear-stress plane in monolithic metallic glasses by taking the normal stress into account. This critical shear-fracture condition can be expressed as [296, 297]:

$$
\tau_{\theta}=\tau_{0}+\mu_{0} \sigma_{\theta}
$$

where $\tau_{0}$ is the critical shear strength on the shear-fracture plane without a normal stress, $\mu_{0}$ is a constant of the material, indicating the effect of the normal stress on the shear-fracture plane, and $\tau_{\theta}$ and $\sigma_{\theta}$ are shear and normal stresses on the shear-fracture plane. According to the rule of mixtures in the fiber-reinforced composite, $\tau_{0}$ in Eq. (24) can be expressed as:

$$
\tau_{0}=\tau_{m}\left(1-V_{f}\right)+\tau_{f} V_{f}
$$


where $\tau_{m}$ and $\tau_{f}$ are critical shear strengths on the shear-fracture plane of the metallic glass matrix and the tungsten fiber, respectively. In addition, shear and normal stresses, $\tau_{\theta}$ and $\sigma_{\theta}$, on the shear-fracture plane of the composite can be written as:

$$
\begin{gathered}
\tau_{\theta}=\sigma_{\text {shear }} \sin \theta_{c} \cos \theta_{c} \\
\sigma_{\theta}=\sigma_{\text {shear }} \sin ^{2} \theta_{c}
\end{gathered}
$$

where $\sigma_{\text {shear }}$ is the compressive fracture strength of the composite failed in a shear mode, and $\theta_{c}$ is the actual shear-fracture angle. Substituting Eqs. (25), (26a), and (26b) into Eq. (24), one can obtain:

$$
\sigma_{\text {shear }}\left(\sin \theta_{c} \cos \theta_{c}-\mu_{0} \sin ^{2} \theta_{c}\right)=\tau_{m}\left(1-V_{f}\right)+\tau_{f} V_{f}
$$

Since the fracture strength of the tungsten fiber is higher than that of Vit1, this critical shear strength, $\tau_{f}$, should be higher than $\tau_{m}$. As a result, the compressive fracture strength, $\sigma_{\text {shear }}$, of the composite will increase with increasing the fiber volume fraction, as schematically illustrated in Figure 33(a) [296]. With increasing the fiber volume, the splitting fracture mode can be easily observed. Besides, multiple shear bands are distributed between the fibers. The failure of the composite can be reflected microscopically by the competitive processes between the shear-band development in the matrix and splitting fracture of the tungsten fiber.

It is supposed that there exist critical shear fracture stresses on the shear-fracture plane, $\tau_{\theta}^{V_{1}}$ and $\tau_{\theta}^{V_{2}}$, for composites with fiber volume fractions of $V_{1}$ and $V_{2}$, respectively $\left(V_{1}<V_{2}\right)$. Shear fracture may occur for the two composites, when the following conditions are satisfied:

$$
\tau_{\theta}^{V_{1}}=\tau_{0}^{V_{1}}+\mu_{0}^{V_{1}} \sigma_{\theta}^{V_{1}}
$$




$$
\tau_{\theta}^{V_{2}}=\tau_{0}^{V_{2}}+\mu_{0}^{V_{2}} \sigma_{\theta}^{V_{2}}
$$

where the superscript denotes the parameters under the $V_{1}$ and $V_{2} \cdot \mu_{0}^{V_{1}}$ and $\mu_{0}^{V_{2}}$ are almost equal, as the fracture angles are very similar. $\tau_{0}$ increases with increasing the fiber volume fraction. Therefore, $\tau_{0}^{V_{2}}$ should be higher than $\tau_{0}^{V_{1}}$. One can obtain two critical shear fracture conditions from Eqs. (28a) and (28b), which are represented by the two lines, AB and CD, as illustrated in Figure 33(b) [296]. Constant $\mu_{0}^{V_{1}}$ and $\mu_{0}^{V_{2}}$ represent the slopes of the two lines. The Mohr circle represents the stress state of the composite under compression. A certain point on the Mohr circle represents normal and shear stresses on a plane. The diameter of the Mohr circle represents the applied compressive stress along the loading axis and will enlarge with increasing the applied load. The specimen will fail in a shear mode, when the Mohr circle touches one of the two critical shear-fracture lines. At this time, the diameters of the two Mohr circles, $\sigma_{\text {shear }}^{V_{1}}$ and $\sigma_{\text {shear }}^{V_{2}}$, in Figure 32(b) represent the compressive fracture strengths of the composite - i.e.,

$$
\begin{aligned}
& \sigma_{\text {shear }}^{V_{1}}=\frac{2 \tau_{0}^{V_{1}}}{\sqrt{1+\left(\mu_{0}^{V_{1}}\right)^{2}}-\mu_{0}^{V_{1}}} \\
& \sigma_{\text {shear }}^{V_{2}}=\frac{2 \tau_{0}^{V_{2}}}{\sqrt{1+\left(\mu_{0}^{V_{2}}\right)^{2}}-\mu_{0}^{V_{2}}}
\end{aligned}
$$

From Eqs. (29a) and (29b), $\sigma_{\text {shear }}^{V_{2}}>\sigma_{\text {shear }}^{V_{1}}$ since $\tau_{0}^{V_{2}}>\tau_{0}^{V_{1}}$. For the composites with a higher fiber volume fraction to fail in a splitting mode, the higher the lateral strain and, therefore, higher compressive stresses are needed. Consequently, the critical stress for the splitting fracture, $\sigma_{\text {split }}$, will increase with increasing the fiber-volume fraction, as illustrated in Figure 33(a). The critical stress for the splitting and shear fracture trend 
lines will intersect at a critical fiber volume fraction, $V_{\mathrm{C}}$, [Figure 33(a)]. As shown in Figure 33(b), the two critical stresses, $\sigma_{\text {split }}^{V_{1}}$ and $\sigma_{\text {split }}^{V_{2}}$, are represented by the two vertical lines, EF and GH, respectively. For the low-volume-fractioned composites, $\sigma_{\text {shear }}^{V_{1}}<\sigma_{\text {split }}^{V_{1}}$. As the applied load increases, the stress Mohr circle, a, will first contact the critical shear fracture line, $\mathrm{AB}$, prior to the critical splitting fracture, line $\mathrm{EF}$, and the composite will fail in a shear fracture mode. For the composites with a volume fraction of fibers of $V_{2}, \sigma_{\text {shear }}^{V_{2}}>\sigma_{\text {split }}^{V_{1}}$. In this case, the stress Mohr circle, $\mathbf{b}$, will first contact the critical splitting fracture line, $\mathrm{GH}$, prior to the critical shear-fracture line, $\mathrm{CD}$, and the composite will fail in a splitting mode. Consequently, shear fracture will occur at $V_{\mathrm{f}}<$ $V_{\mathrm{c}}$, whereas the splitting fracture happens when $V_{\mathrm{f}}>V_{\mathrm{c}}$.

Although tungsten fibers have the super high strength of about $3,500 \mathrm{MPa}$, little ductility can be obtained [298]. In comparison, Ta fibers [65] or stainless steel fibers [65] possess larger ductility, and splitting would not easily occur in these composites.

Besides the long fiber / MGMCs, the mechanical performances of short fibers or particles / MGMCs are attractive. Figure 34 displays the specific fracture strength and compressive plasticity of a series of monolithic BMGs and ex-situ particle-reinforced MGMCs [48]. It is noted that the $\mathrm{Mg}_{65} \mathrm{Cu}_{25} \mathrm{Gd}_{10} \mathrm{MGMC}$ with 40 vol.\% Ti powders is found to have a high specific strength of $3.3 \times 10^{5} \mathrm{Nm} / \mathrm{kg}$ with the remarkable plastic deformation of $40 \%$ [48], suggesting a novel guideline to develop Mg-based alloys having high specific strength with high ductility.

\section{2) Compression of in-situ composites}


As discussed above, the nanocrystal-reinforced MGMCs exhibit notable compressive plasticity, since not only the nanocrystals can act as obstacles to impede the fast propagation of shear bands [74-82], but also the nanoscale heterogeneity in amorphous alloys could act as precursors for the multiplication of shear bands [83-85]. An analogous function is found in dendrites or Ta solid-solution / MGMCs.

Besides, the larger-sized dendrites or Ta particles are easier to absorb the plastic-strain energy than the small-sized nanocrystals, since the severe lattice distortion and local amorphization in the dendrites, as well as pile-ups of dislocations close to the interface between the dendrites and the glass matrix upon straining, are jointly responsible for the accommodation of compressive plastic strains [248]. For such kinds of stable solid solutions, almost no phase transformation happens during plastic deformation $[69,94,95,101]$. There are only a few studies indicating the deformation-induced phase transformation in $\beta$-Ti dendrites [299] and crystallization of the amorphous matrix in the $\left(\mathrm{Cu}_{60} \mathrm{Zr}_{30} \mathrm{Ti}_{10}\right)_{0.95} \mathrm{Ta}_{5}$ composite upon room-temperature compression [300]. Taking $\mathrm{Zr}_{58.5} \mathrm{Ti}_{14.3} \mathrm{Nb}_{5.2} \mathrm{Cu}_{6.1} \mathrm{Ni}_{4.9} \mathrm{Be}_{11.0}$ composites as an example, the compositions of the dendrites and the glass matrix are to be $\mathrm{Zr}_{66.52} \mathrm{Ti}_{15.07} \mathrm{Nb}_{8.06} \mathrm{Cu}_{9.74} \mathrm{Ni}_{0.60}$ and $\mathrm{Zr}_{49.79} \mathrm{Ti}_{12.90} \mathrm{Nb}_{2.85} \mathrm{Cu}_{20.77} \mathrm{Ni}_{13.70}$, respectively [248]. Noted that Be is not detected by EDS, and it is almost all enriched in the glass matrix. Figure 35 shows the compressive engineering stress-strain curves of the composites, dendrites, and glass matrix, respectively. The yielding strengths of the composites, dendrites, and glass matrix are about 1.3, 0.8, and 2.0 GPa, respectively. The high yielding strength and zero plasticity are for the glass matrix, while the low yielding 
strength and large plasticity for the dendrite. The dual-phase composites split the difference, combining the high yielding strength and large plasticity. The yielding strength of the composites is simply calculated by the RoM model:

$$
\sigma_{c}=f_{d} \sigma_{d}+\left(1-f_{d}\right) \sigma_{g}
$$

where $\sigma_{c}, \sigma_{d}$, and $\sigma_{g}$ are the yielding strengths of the composite, dendrite, and glass matrix, respectively, and $f_{\mathrm{d}}$ is the volume fraction of dendrites. Equation (30) yields $\sigma_{c}=1.34 \mathrm{GPa}$, which is in good agreement with the experimental value of $1.3 \mathrm{GPa}$.

For another kind of in-situ MGMCs, transformation-mediated ductilization composites [128, 129], multiple yielding occurs. For Cu-Zr-Al MGMCs, triple yielding dominates, and the mechanisms that are responsible for the triple yielding are as follows: (1) the first yielding results from the yielding of the $\mathrm{B} 2 \mathrm{CuZr}$ phase and the initiation of its martensite transformation (MT); (2) the second yielding originates from the combined action of the further development of MT and the multiplication of shear bands; and (3) the third yielding is due to the formation of a high density of dislocations and partial detwinning [129].

\subsubsection{Compression at high temperatures}

Subjected to the high-temperature surroundings, BMGs or the glass matrix in MGMCs are easily crystallized. The crystallization has a great effect on the mechanical properties of BMGs or MGMCs [70].

\section{1) Compression of ex-situ composites at high temperatures}

Figure 36 shows compressive stress-strain curves at four different test temperatures $(77,294,373$, and $473 \mathrm{~K})$ for 60 vol. $\% \mathrm{~W}_{\mathrm{f}} / \mathrm{Zr}$-based MGMCs [59]. The 
yielding strength and total strain are distinctly influenced by the testing temperatures. The yielding strength is $2,800 \mathrm{MPa}$ at $77 \mathrm{~K}$ and $1,670 \mathrm{MPa}$ at $473 \mathrm{~K}$. The room-temperature yielding strength is $2,100 \mathrm{MPa}$. Obviously, the temperature drastically affects the compressive strength and plastic strain of composites. For the metallic glasses, softening takes place, subjected to high temperatures [301], while for W fibers, decreased yielding points are available at high temperatures. Ultimately, decreased plastic flows with the temperature are obtained.

\section{2) Compression of in-situ composites at high temperatures}

In-situ dendrite / MGMCs as promising materials are rarely studied on their mechanical properties at high temperatures. Actually, the use of in-situ MGMCs in commercial applications requires an understanding of how they behave at elevated temperatures. Presently, the high-temperature deformation of in-situ $\mathrm{Zr}_{39.6} \mathrm{Ti}_{33.9} \mathrm{Nb}_{7.6} \mathrm{Cu}_{6.4} \mathrm{Be}_{12.5}$ composites with $T_{\mathrm{g}}$ of $600 \mathrm{~K}$ is investigated. Figure 37 shows the comparison of the stress-strain responses at various temperatures of testing $(300,550,600$, and $650 \mathrm{~K})$ [302]. From Figure 37(a), at room temperature (300 K), which is well below $T_{\mathrm{g}}$ of the composite, the yield strength is sensitive to the strain rate, but the effect is not as pronounced as that seen at $T=650 \mathrm{~K}$. When the testing temperature is increased to $550 \mathrm{~K}$, i.e., $T<T_{\mathrm{g}}$, the composite's stress-strain response becomes completely rate insensitive with all the plots, as shown in Figure 37(b). When the testing temperature is increased to $600 \mathrm{~K}$, i.e., $T=T_{\mathrm{g}}$, the yielding strength of the composite decreases, relative to room temperature, when tested at a strain rate of $10^{-4}$ 
$\mathrm{s}^{-1}$ in Figure $37(\mathrm{c})$. At $650 \mathrm{~K}$, i.e., $T>T_{\mathrm{g}}$, the positive strain-rate sensitivity prevails from Figure 37(d). Specially, at a strain rate of $10 \mathrm{~s}^{-1}$, softening happens.

In-situ dendrite / La-based MGMCs exhibit homogeneous flows at high temperatures [303]. In those composites, with a low reinforcement volume percentage, the flow behavior is dominated by the properties of the matrix phase. Above the threshold / critical volume fractions of dendrites, the deformation of La dendrites is dominating the composite properties. The dendrite / MGMCs have a higher strength than single amorphous phases at high temperatures due to the dendrite-phase hindering the viscous flow of the glassy matrix, unlike the room-temperature deformation behavior. Besides, Fu et al. [70] have studied the high-temperature deformation behavior of in-situ intermetallics / Zr-Cu-Al compsites. Inhomogeneous flows and apparent brittle fracture were observed at low temperatures, with a transition to the homogeneous flow at high temperatures. During the inhomogeneous deformation, as the volume percentage of the second phase increases, the flow stress increases, from two separate contributions: the load transfer from the amorphous matrix to the reinforcements and direct hardening of the glassy matrix due to changes in the glass composition and structure upon the precipitation of reinforcements [70].

\subsubsection{Compression at low temperatures}

It has been demonstrated that the improved plasticity together with the increased strength could be obtained for monolithic BMGs at cryogenic temperatures, such as at the liquid-nitrogen temperature (77 K) [304-306]. The serrations in the stress-strain curve disappear at $77 \mathrm{~K}$. During shear banding at cryogenic temperatures, 
both the instantly high-temperature rise and the rapid heat conduction enable the disappearance of serrations, avoiding the early failure [306]. Li et al. [304] have found that the normalized strength, $\sigma / E$ (the strength to the Young's modulus), shows a linear relationship with the normalized temperature $\left(T / T_{\mathrm{g}}\right)$, and an equation can be established as:

$$
\sigma=a E\left(b-\frac{T}{T_{g}}\right)
$$

where $\sigma$ is the strength of BMGs, $E$ is Young's modulus, $T$ is the testing temperature, $T_{\mathrm{g}}$ is the glass-transition temperature, and $a$ and $b$ are constants. Equation (31) is of importance to calculate the low-temperature strength.

Mechanical properties of 70 vol. $\% \mathrm{~W}_{\mathrm{p}} / \mathrm{Zr}$-based composites were measured at 693, 298, and $77 \mathrm{~K}$. As shown in Figure 38(a) [307], the plastic strains decrease significantly upon lowering the testing temperature, i.e., over $110 \%$ (a stopped test) at $693 \mathrm{~K}$, about $58 \%$ at $298 \mathrm{~K}$, and $20 \%$ at $77 \mathrm{~K}$, while the yielding strengths increase significantly, i.e., $390 \mathrm{MPa}$ at $693 \mathrm{~K}, 930 \mathrm{MPa}$ at $298 \mathrm{~K}$, and 1,300 MPa at $77 \mathrm{~K}$, respectively. It indicates that ex-situ MGMCs exhibit the increased strength and reduced plasticity at cryogenic temperatures, and analogous cases happen in in-situ Ta solution / MGMCs, as shown in Figure 38(b) [307]. The investigation on Ta-containing composites shows the increased strength with the unchanged plastic strain of $13 \%$ upon lowering the testing temperature from 298 to $77 \mathrm{~K}$. The yielding and maximum strengths obtained at $298 \mathrm{~K}$ are about 1,760 and 1,830 MPa, whereas at $77 \mathrm{~K}$ they are about 2,040 and 2,120 MPa - a $16 \%$ increase. 
By contrast, for the dendrite-reinforced MGMCs, a

distinguishingly-increased maximum strength is available. However, a

ductile-to-brittle transition seems to be present by lowering the temperature to $77 \mathrm{~K}$, which is caused by the ductile-to-brittle transition of the dendrites [308]. Besides, a semi-empirical formula is proposed by Qiao et al. [308] to predict the maximum strength at $77 \mathrm{~K}$ for BMGs and MGMCs, as follows:

$$
\sigma_{\text {max. } .77}=55 \frac{\rho_{0}}{M} \Delta T_{g}+\Delta \sigma
$$

where $\rho_{0}$ is the density, $M$ is the molar mass, $\Delta T_{g}$ is the temperature difference between the glass-transition temperature, $T_{g}$, and room temperature, $T_{0}$, and $\Delta \sigma$ is the average difference of the maximum strengths at 77 and $298 \mathrm{~K}$, with a value of $314 \pm$ $80 \mathrm{MPa}$. Equation (32) is an effective prediction for the low-temperature $(77 \mathrm{~K})$ strength.

\subsection{Deformation of MGMCs under quasi-static tension}

Even if no tensile ductility at room temperature has been found in monolithic BMGs, it is still possible to obtain such properties in MGMCs, since the evolution from shear bands to cracks could be effectively retarded by the secondary phases. Only if the rate by the absorbance of plasticity is larger than that by crack opening, toughness can be definitely achieved in MGMCs under tension.

\subsubsection{Tension at room temperature}

\section{1) Tension of ex-situ composites}

Although a great deal of investigations on compressive properties of ex-situ MGMCs were conducted, studies on tensile properties were scarcely covered. As 
early as in 1998, Conner et al. [57] have examined the tensile behavior of ex-situ tungsten and steel fiber / Vit 1 composites, and it was found that any tensile ductility could be scarcely obtained for tungsten-fiber-reinforced MGMCs, regardless of volume fractions of fibers. In contrast, ductile steel-fiber-reinforced composites possess the macroscopic plastic-deformation ability, when volume fractions of steel fibers are over than $60 \%$. Later, people found final strains of 7 and $17 \%$ for $80 \mathrm{vol}$. \% Ta and 80 vol. \% Mo-fiber-reinforced Zr-based MGMCs, respectively [309]. Similarly, Lee et al. [61] detected a tensile elongation of $7 \%$ in 60 vol. \% Ta fiber / Vit 1 composites. At the meantime, however, almost brittle fracture upon quasi-static tension was the result for 60 vol. \% W fiber composites [61]. It is known that tungsten fibers have a higher strength than typical Zr-based BMGs, such as Vit 1 [310]. But the elongation of composites is hardly enhanced due to the brittleness of tungsten fibers. Consequently, the above excellence in ductile fiber-reinforced MGMCs can be explained in terms of the following mechanisms of thermally stable and ductile reinforcing fibers: (1) blockage of crack propagation, (2) multiplication of shear bands inside the matrix, (3) plastic deformation of fibers, and (4) fracture prevention by bridging effects. Besides, tensile ductility in brittle fiber / MGMCs at room temperature is hardly achieved, since the fiber pullout or fiber / matrix interfacial debonding frequently found in typical fiber-reinforced matrix composites is hardly observed. $[42,57]$.

\section{2) Tension of in-situ composites}


For in-situ MGMCs, the crystalline phases with lower shear moduli than the resulting glasses are distributed within the glass matrices. Upon loading, the propagation of shear bands can be arrested by the soft crystalline phases, the length scale of which matches the plastic-zone size of the glass matrices [94, 95]. As a consequence, the tensile instability can be suppressed, and the significantly-improved toughness prevails.

Figure 39 plots the ultimate strength and tensile ductility of various in-situ MGMCs, including dendrite-reinforced, Ta-solution-reinforced, and transformation-mediated composites in La-, Zr-, Ti-, and CuZr-based alloy systems $[90,94,99,101,103-105,108,109,113,126,132,160,311-313]$. The dendrite-reinforced La-based MGMCs exhibit low strength due to the intrinsic low strength of La [108]. For other kinds of composites, high strengths are easily obtained. It is noted that although the macroscopic tensile ductility is achieved, most these composites soften rather than work harden after yielding. An exception is that the transformation-mediated MGMCs possess the work-hardening capacity upon tension, due to the stress-induced martensitic transformation from $\mathrm{B} 2-\mathrm{CuZr}$ to $\mathrm{B} 19^{\prime}-\mathrm{ZrCu}$ $[126,132]$. Another exception is that the role of $\beta$ phases surrounding $\alpha$ phases prevents the formation of twins, and, thus, work hardening occurs in dendrite composites $[103,311]$. Homogeneous deformation together with work hardening is of importance for engineering structural materials, avoiding early localized failure. Therefore, how to design MGMCs with distinguished work hardening upon tension at room temperature would draw more attention in the scientific and technical fields. 


\subsubsection{Tension at high temperatures}

For actual structural engineering applications of MGMCs, many kinds of extreme conditions other than quasi-static loadings at room temperature should be appreciated. For instance, the high-temperature mechanical behavior, such as in the supercooled liquid region (SLR), is scarely studied. Previously, the superplastic deformation of monolithic BMGs and superplastic formability are widely observed, although nanocrystals precipitate after high-temperature deformation [314-316]. These BMGs are observed to exhibit Newtonian behavior at low strain rates but to become non-Newtonian at high strain rates $[314,315]$. It is not clear what would be the flow behavior, when the volume fraction of secondary phases is higher in contrast to the very low-volume fractioned nanocrystals, precipitated in monolithic BMGs.

Figure 40(a) presents the tensile true stress-strain curves of ex-situ porous tungsten / Zr-based metallic-glass interpenetrating composites, with $T_{\mathrm{g}}$ of $628 \mathrm{~K}$ and $T_{\mathrm{x}}$ of $701 \mathrm{~K}$, spanning a wide range of temperatures at a strain rate of $1 \times 10^{-4} / \mathrm{s}$ [317]. The inset is the microstructure with a volume fraction of $\mathrm{W}$ phases of $80 \%$, and the morphology of amorphous phases (dark) is irregularly distributed. At room temperature, the composite experiences brittle fracture. When the temperatures vary from $618 \mathrm{~K}$ to $718 \mathrm{~K}$, all curves show the stress overshoot at the first deformation stage, followed by obvious "work-hardening" phenomena [317]. The W phase dominates the deformation processes due to its high volume fraction and strength. The existence of amorphous phases is beneficial to the deformation and formability in the supercooled liquid region. 
Figure 40(b) displays the stress-displacement curves of in-situ dendrite / Ti-based MGMCs at $613 \mathrm{~K}$ with a volume fraction of dendrites of $43 \%\left(T_{\mathrm{g}}\right.$ being about $603 \mathrm{~K}$, and the SLR being about $30 \mathrm{~K}$ ) [318]. The composites exhibit softening after yielding and expected tensile ductility in the SLR. The inset in Figure 39(b) presents the pictures of the tensile specimens before and after tension. Obviously, the local necking occurs, and the specimens are drawn to a point, which gives the evidence of softening. The average tensile strength is about $970 \mathrm{MPa}$ at a high temperature of $613 \mathrm{~K}$, compared to the $1,640 \mathrm{MPa}$ at room temperature $[101,318]$. The presence of dendrites within the glass matrix distinguishingly increases the viscosity of the composites at high temperatures. Consequently, the superplastic elongation is absent for $i n$-situ dendrite composites.

Figure 40(c) shows the tensile true tress-strain curves at $693 \mathrm{~K}\left(T_{\mathrm{g}}\right.$ of $669 \mathrm{~K}$ and SLR of $94 \mathrm{~K}$ ) for the in-situ Ta-particle-reinforced MGMCs with a composition of $\mathrm{Zr}_{55.9} \mathrm{Cu}_{18.6} \mathrm{Ni}_{10} \mathrm{Al}_{7.5} \mathrm{Ta}_{8}$ (at. \%) [319]. All curves are characterized by a stress overshoot in the initial stage of deformation, followed by a steady-state flow with a stress plateau in the extended strain region. The peak of the stress-overshoot increases, as the strain rate increases, while the steady flow stress seems to be independent of the strain rate with an average value of about $25 \mathrm{MPa}$. It is noted that super elongation is obtained regardless of strain rates, as shown in the inset, similar to that in the monolithic BMGs. In contrast to the behavior in dendrite-reinforced MGMCs [318], the low-volume-fractioned Ta solution of $10 \%$, as exhibited in the inset, could not effectively withstand the viscous flow of the glass matrix in the SLR. 
Besides, superplastic compression is achieved for this kind of composites [320]. Marandi et al. [321] recently developed a constitutive description of in-situ dendrite / La-based MGMCs, which is able to describe the stress-strain responses observed, and there was a good correlation with the experimental data.

\subsubsection{Tension at low temperatures}

Although the enhanced plasticity, together with the increased strength, is obtained for the monolithic BMGs upon low-temperature compression [304-306], the tensile ductility is not improved [216]. It was observed that the macroscopic fracture mode varies from the ductile shear fracture to brittle normal tensile fracture, and the microscopic fracture feature changes from the micron-scaled vein patterns to nano-scaled dimples with decreasing test temperatures, which indicate a significant ductile-to-brittle transition (DBT) behavior. What would happen to dual-phase MGMCs when the secondary crystalline phase exists is not clear.

Figure 41(a) presents the tensile stress-strain curve of in-situ dendrite MGMCs with a composition of $\mathrm{Ti}_{48} \mathrm{Zr}_{20} \mathrm{Nb}_{12} \mathrm{Cu}_{5} \mathrm{Be}_{15}$ at $77 \mathrm{~K}$ [322]. The tensile strength and ductility are about 2,200 $\mathrm{MPa}$, and $8 \%$, respectively. In comparison, upon the quasi-static compression at $77 \mathrm{~K}$, the plasticity can attain $15 \%$ [323], much larger than that upon tension. The volume fraction of bcc dendrites is approximately $56 \%$, and the average span and the interval of neighboring dendrites [102] are 15 and $10 \mu \mathrm{m}$, respectively [323]. The pictures of samples before and after deformation are shown in Figure 41(b), and it is observed that obvious necking is present, in agreement with softening in Figure 41(a). In contrast, work hardening dominates upon 
compression. It is the first to obtain the low-temperature tensile ductility in in-situ dendrite reinforced MGMCs. The high-volume-fractioned and coarsened dendrites facilitate the tensile ductility even at low temperatures. Besides, phase-transformation-induced ductility can be ruled out, since it has been demonstrated that only diffraction patterns from the bcc phase after deformation can be detected, as shown in Figure 41(c). Analogous diffraction patterns have been widely reported [101, 105, 318]. Although the tensile ductility can be achieved in such kind of MGMCs, it is not clear whether other kinds of MGMCs exhibit similar mechanical behavior.

\subsection{Modeling}

Although MGMCs show enhanced ductility in experiments [89, 94, 101], the microstructural evolution of these MGMCs are yet to be investigated in detail, especially on the atomistic scales. At present, the understanding of these deformation mechanism topics relies mainly on post-mortem microstructure observations in experiments, such as, Narayan et al. [324] showed that larger dendrite sizes led to higher ductility, compared with smaller ones, whereas finer interdendritic spacing results in significant improvements in both the ductility and yield strength of MGMCs due to the confinement of shear-band nucleation in the glass matrix. Recently, more and more simulations work has been proved to be an excellent complement to experimental results, which allows one to explore the distinct properties of MGMCs that are not accessible to experiments. For example, the large-scale molecular-dynamics (MD) and phase-field models can be employed to investigate the effects of secondary crystalline 
phases on the structural evolution of MG-matrix composites from the atomistic scale, including different inclusion shapes, volume fractions, crystalline orientations, residual stresses, and atomic bonding conditions, as discussed below [325-327].

\subsubsection{Shape effects}

The MD model is setup in the binary $\mathrm{MG}$ alloy $\mathrm{Cu}_{64} \mathrm{Zr}_{36}$ (at.\%). By inserting discontinuous $\mathrm{Cu}$ particles into the $\mathrm{Cu}_{64} \mathrm{Zr}_{36}$ glass matrix, a mixed structure can be generated, with a typical geometry of this composite illustrated in Figure 41(a) [327]. By adjusting these structural parameters, various patterns of $\mathrm{Cu}$ nanocrystal- enhanced MGMCs can be generated as illustrated in Figure 42 [327]. Tension simulations are performed on three composites (I, II, and III) with different geometries of nanocrystals by adjusting the aspect ratio $\mathrm{b} / \mathrm{a}(6.5,5.7$, and 3.7 , respectively).

The stress-strain curves of this set of MGMCs shows that the tensile stresses of MGMCs increase linearly with the applied strain in Ref. [327]. Then, an abrupt stress drop appears after global yielding due to the STZ localization and the formation of shear bands near structural inhomogeneities, such as free surfaces and glass / crystal interfaces, resulting in shear-banding-induced strain softening in the amorphous matrix [220]. Local sites with more free volumes tend to be sheared at a lower stress, which contributes to the initiation of individual STZs in the glass matrix, resulting in shear bands with the thickness of $\sim 10 \mathrm{~nm}[36,260,327]$. At relatively-large strains, the sudden stress drops on the stress-strain curves of MGMCs appear, which is attributed to the penetration of an operating shear band through the crystalline inclusions, as illustrated in Figure 42(c). 
To contrast the behaviors of the composites and the monolithic MG sample, the typical deformation patterns of Composites I and III, and Monolithic MG are exhibited in Figures 42(d) and (e). From Figure 42(d), it can be observed that shear bands have been greatly suppressed by the nanocrystals (indicated by the arrow), when Composite I is deformed at a strain of $15 \%$. For the monolithic MG, a shear band initiates from the left free surface and then traverses the entire length of the MG sample without any resistance at a strain of $10 \%$ in Figure $42(\mathrm{e})$, which is the typical deformation mechanism of monolithic MGs.

Similar mesoscaled simulation results are also reported on the deformation of MGMCs with different geometries, as shown in Figure 43 [326], which display the representative microstructures of Comp1, Comp2, and Comp3. In Comp1, dendrites are represented by dispersed disks, while in Comp2, the more randomly-distributed dendrites form spatially-disconnected regions. In Comp3, the dendrites are highly stretched ellipses that form a connected framework. Figure 43 (a)-(c) show the strain contours of Comp1, Comp2, and Comp3, respectively, at a nominal strain of $11.5 \%$ [326]. It can be observed that a fully-developed shear band exists in Comp1, while several shear bands started to form and propagate in Comp2, implying extensive plasticity prior to the catastrophic failure. In Comp3, strain is still globally distributed without a sign of shear-band initiation.

From the discussion above, two mechanisms are responsible for the enhanced global plasticity of MGMCs, compared with the monolithic MG. Firstly, due to the stress concentration and strain mismatch around crystalline inclusions, STZ 
operations in the glass matrix are promoted. Second, crystalline inclusions with appropriate geometry provide effective barriers to shear-band propagation.

\subsubsection{Volume-fraction effects}

Figure 44 shows the stress-strain curves of composites with various crystalline volume fractions of $24.2 \%, 33.3 \%, 43.5 \%$, and $50.1 \%$, respectively [327]. It can be observed that the yield strength of MGMCs is slightly lower than that of monolithic specimens. This stress drop of the global yielding stress becomes larger as vol. \% increases from $14.5 \%$ to $50.1 \%$. However, it can also be observed that the ultimate tensile stress (UTS, the first peak stress on the stress-strain curve) of the composites decrease first, compared to the monolithic MG, and then goes up, as the vol. $\%$ increases. For instance, the UTS decreases from $\sim 4.6$ to $\sim 4.1 \mathrm{GPa}$, as vol. $\%$ increases from $0 \%$ (Monolithic MG) to $24.2 \%$ (Composite IV). However, the UTS goes up to $\sim 5.1 \mathrm{GPa}$ as the fv increases to $50.1 \%$ (Composite VII), which is even greater than that of the monolithic MG.

The atomistic mechanism for this phenomenon can be revealed by examining the deformation process of 'Composite VII' in Figure 44(b)-(d) [327]. It can be observed that no shear band appears under the strain of $13 \%$ [Figure 44(b)], $15 \%$ [Figure 44(c)], and $16 \%$ [Figure 44(d)], since the deformation mechanisms change as the vol. \% increases. Because almost all of the nanocrystals have been plastically deformed, which provides an efficient delocalizing mechanism that helps transmit shear distortion from one glass subdomain to another. Therefore, in contrast to the shear-localization dominated global yielding for low vol. \% composites (e.g., 
'Composite II' with vol. $\%=14.5 \%$ ), the deformation mechanism of global yielding of high fv composites (e.g., in 'Composite VII' with vol. \% $=50.1 \%$ ) is governed by the activation of lattice slipping in the crystalline second phase, as illustrated in Figure 44(e) and (f). Therefore, optimizing the volume fraction of the crystalline inclusions is extremely important in enhancing the global plasticity of MGMCs.

\subsubsection{Orientation effects}

Three composites consisting of $\mathrm{Cu}$ nanocrystals with various orientations but the same aspect ratio $(\mathrm{b} / \mathrm{a}=5.7)$ are constructed, as shown in Figures 42(f)-(h) [327]. The crystalline pattern of 'Composite Inclined' is inclined to the Y direction at $20^{\circ}$, while that of 'Composite Random' is composed of nanocrystals randomly distributed and oriented in the glass matrix, which is designed to mimic the geometry of the dendritic trees obtained in experiments [86]. Since good plasticity of MGMCs relies on the efficiency in blocking the shearing paths in the glass matrix, the crystalline shape and orientation are two important factors in designing MGMCs. For 'Composite Inclined' with $\theta=20^{\circ}$ (i.e., the tension direction inclined to the crystalline phase), the stress drop induced by global yielding in the stress-strain curve is significant, compared with the case of $\theta=0^{\circ}$, and the deformation pattern is exhibited in Figure 42(f) [327] at a strain of $15 \%$. It can be noticed that a cross-sectional shear band is formed. For the deformation of 'Composite Random', two major shear bands (labeled ' 1 ' and ' 2 ') occur in the composite at a strain of $10 \%$, as indicated by the dashed lines in Figure 42(g) [327]. Upon interactions with the secondary crystalline phase, the local shear bands propagating along 'path 1' penetrate through the 
crystalline inclusions and merge together to form a dominant shear band, while the shear band along 'path 2' disappears, as shown in Figure 42(h), which is consistent with the experimental studies observed on SEM micrographs [86]. From the deformation patterns of 'Composite Dendritic' in Figure 42(g) [327], it is observed that shear localization is greatly restricted by the dendritic inclusions. Once nucleated in a dendritic tree, dislocations will move along the glide planes until being absorbed by the surrounding glass matrix. Therefore, the crystalline dendrites, as well as the orientation effects, play an important role in the structural evolution of the MGMCs.

\subsubsection{Residual stress and atomic-bonding conditions}

The MGMC simulation model used in this section consists of cylindrical reinforcing tungsten fibers with a diameter of $0.2 \mu \mathrm{m}$, which are embedded in a Zr-based BMG $\left(\mathrm{Zr}_{41} \mathrm{Ti}_{14} \mathrm{Ni}_{10} \mathrm{Cu}_{12.5} \mathrm{Be}_{22.5}\right)$ plate with edged notches, as shown in Ref. [325]. In experimental studies [325], the tensile hoop stress around the interface would promote the cracks to propagate toward the reinforcing fibers, while the compressive hoop stress should deflect cracks away from the fibers. The current phase-field simulation would provide further information on how shear-band propagation affected by these two opposite residual stresses at the matrix / inclusion interface.

The effects of residual stresses, as well as atomic-bonding conditions at the matrix/inclusion interface, on shear banding and crack propagation can be summarized into several deformation modes, as illustrated in Figure 45 [325]. Mode I: Perfect adhesion $(\mathrm{w}=0)$ and no residual stress $(\mathrm{g}=0)$ at the matrix/reinforcement 
interface, cracking inside the crystalline reinforcement appears caused by shear band touching the interface, illustrated in Figure 45(a) and (b). Increasing the applied load, the shear band is triggered to split in front of the inclusion to suspend more plastic strains, as shown in Figure 45(b). Mode II: With the compressive residual stress ( $\mathrm{g}=$ $435 \mathrm{MPa}$ ) at the matrix / inclusion interface, shear bands tend to occur along the surface of the reinforcement, as exhibited in Figure 45(c). Mode III: With the tensile residual stress $(\mathrm{g}=175 \mathrm{MPa})$ at the interface, the shear band propagates straightly before it touches the interface, resulting in the cracking inside the inclusion, as displayed in Figure 45(d). Mode IV: For the interface with defective atomic bonding condition $(0<\mathrm{w}<1)$, the shear band would split into two branches and propagate around the reinforcing fiber as illustrated in Figure 45(e). Mode V, in the worse atomic-bonding condition at the interface $(\mathrm{w}=1)$, the shear-band propagation immediately transfers from one side of the reinforcement to the other side around the inclusion, as shown in Figure 45(f).

Besides, at the continuum level, a two-phase finite-element model by Qiao [105] quantitatively describes macroscopic MGMC deformation mechanics. The model is based on a five-step deformation regime, which starts with the pure elastic deformation, then adds plasticity in the crystalline phase, then goes through three stages where both phases yield, then the crystallites harden, and finally the composite softens and fails. The examination of shear band-inclusion interactions indicates that the nucleation and diffuse transmission of numerous shear bands is critical to the strain delocalization [328]. 
The current simulation results can quantitatively describe the occurrences

of these deformation modes by characterizing the residual stresses and atomic-bonding condition at the matrix / reinforcement interface. Therefore, this simulation approach is very useful in the development and design of MGMCs with the improved fracture toughness and ductility. 


\section{Deformation of metallic glass matrix composites under uniaxial dynamic loading}

\subsection{Dynamic compression and dynamic tension of BMGs}

As discussed above, usually, the mechanical performances are studied under quasi-static loading including quasi-static compression and tension. In engineering structural applications, not all the loadings on structural materials are quasi-static, and dynamic loadings often exerted on them [329, 330]. Usually, the strain rates are higher than $10^{2} / \mathrm{s}$, and they can be achieved through the Split-Hopkinson pressure bar (SHPB) and shock impact, etc. Regardless of BMGs upon dynamic compression or tension, almost brittle failure results, associated with adiabatic shearing [215, 331-336]. Under adiabatic circumstances, the increase of free volumes at the initial stage and the subsequent significant temperature rise were believed to accelerate strain softening of BMGs. But for conventional crystalline alloys, such as Ti and Al alloys, etc., unusual plastic flows come about, since there is enough time for the multiplication of dislocations [329, 337].

In general, the temperature change and the applied mean stress that cause the volumetric dilatation can be linked in the following form [215]:

$$
\Delta T=\frac{\sigma_{m}}{3 \alpha B}
$$

where $\sigma_{m}$ is the superimposed mean stress (negative under tension), $B$ is the bulk modulus, and $\alpha$ is the thermal expansion coefficient. For a typical Zr-based BMG, $\mathrm{Zr}_{56.7} \mathrm{Cu}_{15.3} \mathrm{Ni}_{12.5} \mathrm{Nb}_{5.0} \mathrm{Al}_{10.0} \mathrm{Y}_{0.5}, \sigma_{m}$ is $\sim 2.3 \mathrm{GPa}, B$ is $\sim 117 \mathrm{GPa}$, and $\alpha$ is $\sim 10^{-5} \mathrm{~K}^{-1}$ [215]. Here, the temperature change is $\sim 950 \mathrm{~K}$, greatly higher than $T_{\mathrm{g}}$ of $670 \mathrm{~K}$ [215]. It 
is reasonable to deduce a rapid drop in the viscosity within shear bands, favoring the nucleation of vein patterns. Similar results are broadly populated in monolithic BMGs [215, 331-336]. Acceleration of shear banding under high-speed dynamic loading makes BMGs fail very early. The existence of secondary phases may alleviate the fast propagation of shear bands. The spread discussion is as below.

\subsection{Dynamic compression of ex-situ composites}

Up to now, the studies on dynamic loading of MGMCs widely focus on dynamic compression on ex-situ MGMCs [65, 66, 338-342]. Other loading modes or dynamic loading on in-situ composites are scarcely investigated. Almost all the ex-situ MGMCs have a distinctly-improved plastic strength upon dynamic compression than that upon quasi-static compression. For the tungsten fiber or porous tungsten reinforced MGMCs, the positive rate sensitivity of the fracture strength is attributed to the large volume fraction of $\mathrm{W}$, which is known to be highly rate sensitive [338]. Due to the high strength of $\mathrm{W}$ and Ta reinforcements, the dynamic-deformation behavior is dominated by shear banding in the glassy matrix, cracking along the phase boundaries, and severe deformation of $\mathrm{W}$ or Ta reinforcements [65, 338-342], similar to the behavior happening under quasi-static compression [343].

However, in the stainless-steel-continuous-fiber reinforced composites with a diameter of $110 \mu \mathrm{m}$ for fibers, the shear fracture occurs rarely, and the complete fracture does not take place even when the fibers are severely buckled [66]. These fibers could interrupt the propagation of the cracks initiated in the matrix, and they promote the continuous deformation without fracture according to the strain-hardening 
effect of the fibers themselves [66]. Figures 45(a) and (b) show the overall morphologies of the stainless steel (S) and tantalum (T) specimens after dynamic compression [65]. The S specimen is super-compressed into a plate, and the fibers are curved severely and dispersed in a radial shape. The T specimen, where the shear fracture has taken place, is broken into many small pieces. Unlike the $\mathrm{S}$ specimen, the $\mathrm{T}$ specimen has a smooth fracture surface, and pulled-out fibers are not observed. With the increase of fiber diameters, such as in the 250 - $\mu \mathrm{m}$-diameter-S-fiber-reinforced composites, fibers are not cut by shear cracks, because the fiber diameter is large enough to restrict the propagation of shear cracks [66]. This composite has the higher yielding and maximum compressive strengths and plasticity than the small diameter-fiber-reinforced composite because of the sufficient ductility of S fibers, the effective interruption of propagation of shear cracks, and the strain hardening of fibers themselves [66]. Based on the above research, superplasticity is expected upon dynamic compression for ex-situ MGMCs, which have high volume fractioned and large-diameter fibers.

\subsection{Dynamic compression of in-situ composites}

As early as in 2006, Lee et al. [344] have conducted dynamic compression on in-situ dendrite-reinforced MGMCs, and found that the maximum compressive strength upon dynamic loading is obviously lower than that upon quasi-static loading.

Later, we have investigated the mechanical behavior of a series of in-situ Zr- and Ti-based MGMCs $[90,158,172,173]$, and all the studied composites unveil brittle failure upon dynamic compression. Furthermore, the absence of plasticity generally, 
observed for the dynamically-fractured samples, may be due to the insufficient time for the formation of multiple shear bands and low resistance to fracture [173].

Interestingly, the failure strength under dynamic loading is approximately equal to the yielding strength under quasi-static compression for such kind of dual-phase composites [173]. Very recently, Chen et al. [345] found a similar shear banding behavior in an in-situ dendrite / Zr-based MGMCs. Based on the calculation, the mismatch between the shear-band toughness of these two phases decreases with increasing strain rate. The shear-band toughness in dendrites is much higher than that in the amorphous matrix in the quasi-static condition, while these values become close for the dynamic situation [345]. Therefore, it is reasonable to deduce a brittle fracture upon dynamic loading.

However, a breakthrough comes about. Striking plasticity upon dynamic compression for dendrite / Ti- and Zr-based MGMCs attains [346-348]. The common feature in these composites, which possess the plastic-flow ability at room temperature, is the special microstructure, characterized by both coarsen and high-volume fractioned dendrites and "ductile framework" [346, 347]. Even the work-hardening capacity under dynamic compression is programmable by tailoring the microstructure [346].

Here, an example is shown. Ti-based in-situ composites have a normal composition of $\mathrm{Ti}_{62} \mathrm{Zr}_{12} \mathrm{~V}_{13} \mathrm{Cu}_{4} \mathrm{Be}_{9}$ with a diameter of dendritic arms of 3 - $5 \mu \mathrm{m}$, and the volume fraction of dendrites is about $60 \%$ [348].

Figure 47(a) [348] displays the engineering stress-strain curve of the $\mathrm{Ti}_{62} \mathrm{Zr}_{12} \mathrm{~V}_{13} \mathrm{Cu}_{4} \mathrm{Be}_{9}$ in-situ MGMCs upon quasi-static compression with the strain rate 
of $10^{-3} / \mathrm{s}$. The yielding strength is about $1,350 \mathrm{MPa}$, and the plasticity prior to failure is $38.4 \%$. It is worth noting that the approximately linear work-hardening behavior dominates, once the yielding happens until the final fracture. Figure 47(b) [348] shows the fractograph of the deformed samples upon quasi-static compression. It is deduced that the final fracture is along the primary shear bands with a shearing angle of about 45 deg. with respect to the loading direction. The magnified deformation region near the crack is presented in Figure 47(c) [348]. Numerous shear bands with a spacing of about $2 \mu \mathrm{m}$ are homogeneously distributed on the lateral surface of the deformed samples, which is in accordance with the large compressive plasticity.

Figure 47(d) [348] exhibits the engineering stress-strain curves upon high-speed dynamic compression with varying strain rates in the range of $1,400-2,500$ /s. It is amazing to note that all the composites upon varied dynamic loading exhibit plastic flows, and most of tested samples are not broken. Instead, the barreling happens, indicating the macroscopic plastic deformation, and stable flows prevail during barreling. Here, the maximum plastic-flow stresses are simply taken as the yielding stresses. Then, the yielding stresses are over than 1,525 MPa, distinguishingly higher than the yielding stress of 1,350 MPa upon quasi-static compression. The increased yielding stress, together with remarkable plastic flows, renders this in-situ dendrite / MGMCs promising as engineering materials under dynamic loading.

Figure 47(e) [348] presents the SEM image of the magnified lateral surface. Multiple shear bands are distributed over the whole lateral surface. The obvious barreling, shown in the inset of Figure 47(e), gives an evidence of plastic deformation. 
Microscopically, the ductile dendrites accommodate plasticity, characterized by the multiplication of dislocations and lattice distortions, together with the appearance of Moiré patterns [348].

Here, the Johnson-Cook (J-C) plasticity model [349] is employed to model the flow behavior of this composite. The J-C model represents the von Mises flow stress, $\sigma$, as a function of the equivalent plastic strain, $\varepsilon$, equivalent plastic strain rate, $\dot{\varepsilon}$, and temperature, $T$, as follows:

$$
\sigma=\left(A+B \varepsilon^{n}\right)\left(1+\ln \dot{\varepsilon}^{*}\right)\left[1-\left(T^{*}\right)^{m}\right]
$$

where $A, B, C$, and $m$ are constants, $n$ is strain hardening exponent, $\dot{\varepsilon}^{*}$ is the normalized equivalent plastic strain rate (typically normalized to a plastic strain rate of $10^{-3} /$ s $)$, i.e., $\dot{\varepsilon}^{*}=\dot{\varepsilon} / \dot{\varepsilon}_{0}\left(\dot{\varepsilon}\right.$ and $\dot{\varepsilon}_{0}$ are the applied strain rate and the referred strain rate, respectively), and $T^{*}$ is the homologous temperature defined as:

$$
T^{*}=\left(T-T_{\text {room }}\right) /\left(T_{\text {melt }}-T_{\text {room }}\right)
$$

where $T$ is the material temperature, $T_{\text {melt }}$ is the melting temperature, and $T_{\text {room }}$ is the room temperature. In the current analysis, the dynamic compression occurs at room temperature. Thus, the temperature effect is ignored. As a consequence, Eq. (35) can be rewritten as follows:

$$
\sigma=\left(A+B \varepsilon^{n}\right)\left(1+\ln \dot{\varepsilon}^{*}\right)
$$

By fitting the flow curves in Figure 47(d) based on the J-C model, the constitutive relationship for the current in-situ dendrite / MGMCs is obtained as: 
$\sigma=\left(1350+54 \varepsilon^{-0.03}\right)\left(1+0.003 \ln \dot{\varepsilon}^{*}\right)$. The experimental and theoretical values become very close. The J-C model plays an important role in predicting the flow stress upon dynamic compression for this kind of dendrite / MGMCs.

Besides, the dynamic compression of $\mathrm{B} 2 \mathrm{CuZr} / \mathrm{Cu}_{46} \mathrm{Zr}_{46} \mathrm{Al}_{8}$ MGMCs has been studied in our group [322]. Previously, the phase-transformation-induced plasticity has been widely found in in-situ CuZr-based BMGMs ductilized by a shape memory $\mathrm{CuZr}$ (B2) phase $[127,129,132]$. It is worth noting that almost all the mechanical testing is under quasi-static tension and compression, and the high-speed loading to such composites has yet to be investigated. It is first to conduct the dynamic compression to $\mathrm{Cu}_{46} \mathrm{Zr}_{46} \mathrm{Al}_{8}$ MGMCs, synthesized by suction casting with a diameter of $5 \mathrm{~mm}$, and the stress-strain curves are exhibited in Figure 48 [322]. When the strain rates are lower than $\sim 10^{3} / \mathrm{s}$, only elastic deformation happens, and the composites have not entered into the plastic deformation. By contrast, when the strain rates are higher than $\sim 10^{3} / \mathrm{s}$, both elastic and plastic deformations take place. Although these composites exhibit high strengths, the scattered strengths exist upon dynamic loading, and a similar phenomenon is found in brittle Mg- and La-based BMGs [350]. Remarkably, a plastic strain of about $1 \%$ can be found, different from the brittle fracture for Mg- and La-based BMGs, indicating the occurrence of phase transformations upon dynamic loading. It has been demonstrated that during deformation of $\mathrm{CuZr}$-based composites, polymorphical nanocrystals precipitate and subsequently these nanocrystals undergo twinning [127]. The formation of such 
structural heterogeneities hampers shear-band generation and results in macroscopically detectable plastic strains.

\subsection{Dynamic tension of ex-situ composites}

As discussed above, almost brittle rupture occurs for monolithic BMGs upon dynamic compression and tension. However, very recently, we found a certain tensile ductility of about $1 \%$ for Vit 1 BMGs upon dynamic tension with a strain rate of about 620 / s, as displayed in Figure 49(a) [322]. Profuse shear bands are distributed on the lateral surface of the deformed samples, as shown in the inset in Figure 49(a). The gauge diameter of BMG samples is about $2 \mathrm{~mm}$. Without doubt, this is first time to obtain tensile ductility in BMGs under high-speed dynamic tension. The detailed deformation mechanism should be focused on further studied.

In contrast to the substantial compressive deformation behavior of MGMCs investigated, there are limited data to reveal the tensile properties of the composites, especially the dynamic tensile deformation behavior. Wu et al. [351] reported that there existed significant asymmetry phenomena, including failure mode, failure stress, and plasticity under compression and tension for Zr-and Ti-based MGMCs, indicating that it is essential to investigate the tensile behavior of MGMCs for drastically understanding the failure behavior of BMGs or MGMCs.

It has been verified that the $\mathrm{Pd}_{40} \mathrm{Ni}_{40} \mathrm{P}_{20}$ BMGs behave brittle fracture upon dynamic tension, remarkably similar to the behavior upon quasi-static tension [352]. What would happen for ex-situ porous W / MGMCs under dynamic tension? Figure 49(b) displays the true stress-strain curves of ex-situ porous W / MGMCs under tension 
at different strain rates [353]. Although the fracture strength increases with strain rates, this kind of ex-situ composites behaves as the brittle rupture. Cavities and cracks are widely observed on the fracture surface, as shown in the inset of Figure 49(b) [322]. The cracks mainly prefer to propagate through the $\mathrm{W}$ phase (marked by rectangle) or along the interface between the metallic-glass phase and the W phase (marked by ellipses) rather than through the metallic glass phase [353]. The study reminders us of avoiding the usage of such kinds of composites upon dynamic tension. In contrast, high-strength flexible laminates [354] give a response to plastically strain upon dynamic tensile loading due to strain rate hardening.

\subsection{Hypervelocity impact of MGMCs}

Ascribed to striking mechanical properties, BMGs and MGMCs are particularly attractive in military and space applications, where this kind of advanced material may be subject to the high-velocity impact during service. Therefore, it is of essence to study the deformation behavior under high strain rates to clarify their underlying deformation mechanisms and widen their practical applicability. Up to now, the strain rates produced by the SHPB technique are usually limited within $10^{2}-10^{3} / \mathrm{s}$, which are insufficient to deeply probe the deformation behaviors of BMGs subjected to the hypervelocity impact, for instance impacted by space debris or micrometeoroids in space exploration.

Nowadays, a few investigations have addressed the high impact velocity shock response of monolithic BMGs $[355,356]$. The plate-shaped $\mathrm{Zr}_{51} \mathrm{Ti}_{5} \mathrm{Ni}_{10} \mathrm{Cu}_{25} \mathrm{Al}_{9}$ BMG samples were impacted at the velocities of $1.40,2.59,3.36$, and $4.27 \mathrm{~km} / \mathrm{s}$ with 
the spherical aluminum projectiles launched by a two-stage light gas gun [356]. It is found that the damage morphologies of the post-impact samples present three different regions: melting area, owing to the kinetic energy of projectile converted into heat; vein-pattern area, attributed to the failure of the sample in a compression-shear mode during the impact process; and a radiate core feature area, caused by the occurrence of spallation under tensile stress [356]. Besides, no phase transformation takes place after hypervelocity impact [356].

For the ex-situ MGMCs, as early as in 2000, ballistic tests have been performed to fire $85 \%$ (vol. \%) $\mathrm{W}_{\mathrm{f}}$ / Vit 1 MGMCs into 4130 steel and 6061 T651 aluminum targets. The composite rods failed by localized adiabatic shear banding and exhibited self-sharpening behavior [357]. The penetrator performance was $10-20 \%$ better than the tungsten-heavy-alloy penetrators of comparable aspect ratios [58, 357]. The $\mathrm{W}_{\mathrm{f}} / \mathrm{Cu}_{82} \mathrm{Al}_{10} \mathrm{Fe}_{4} \mathrm{Ni}_{4}$ composites bear similar good self-sharpening property to the above composites, since the failure mode of the composite projectile during penetration is the rapid peeling of tungsten fibers from the projectile [358]. Meanwhile, it can be found that the microstructure-morphology change of $\mathrm{W}_{\mathrm{f}} / \mathrm{Cu}_{82} \mathrm{Al}_{10} \mathrm{Fe}_{4} \mathrm{Ni}_{4}$ composites occurs after the hypervelocity impact [358]. The long rod penetration experiments of tungsten-fiber / Zr-based metallic glass matrix composite (WF/Zr-MG) and tungsten heavy alloy (93W) into Q235 steel targets are conducted by employing H100 smooth bore artillery within the speed range from $765 \mathrm{~m} / \mathrm{s}$ to $1,809 \mathrm{~m} / \mathrm{s}$ [359]. The experimental results indicate that the $\mathrm{WF} / \mathrm{Zr}-\mathrm{MG}$ rod has much better penetrating performance than the $93 \mathrm{~W}$ rod, when the penetration velocity exceeds $1,000 \mathrm{~m} / \mathrm{s}$. The deformation and 
shear failure of the WF/Zr-MG composite mainly occurs in the edge layer of the rod nose during the high-speed penetration, and it displays the localizing and sharpening features.

The difference in spall behavior between the Vit 1 and in-situ dendrite-reinforced composite $\left(\mathrm{Zr}_{56.3} \mathrm{Ti}_{13.8} \mathrm{Cu}_{6.9} \mathrm{Ni}_{5.6} \mathrm{Nb}_{5.0} \mathrm{Be}_{12.5}\right)$ was studied by Zhuang et al. [360] The spalling in Vit 1 was induced by shear localization, while in $\beta$ dendrite composites, it was due to debonding of the $\beta$ phase boundary from the matrix. The spall strengths at strain rate of $2 \times 10^{6} / \mathrm{s}$ were determined to be 2.35 and $2.11 \mathrm{GPa}$ for Vit 1 and the composite, respectively [360]. The more brittle a BMG is, the more likely it is to experience severe spalling. Hamill et al. [361] have also investigated the spalling behavior of in-situ dendrite-reinforced MGMCs. Vit 1 is subject to poor shock-spalling behavior, even resulting from impacts with small Al projectiles. However, the in-situ composites are shown to have excellent combinations of hardness and toughness for use as shields.

\subsection{Comparison among bulk metallic glasses and composites, and other materials}

Lacking dislocations in BMGs, it is impossible to reach steady-state plastic flows upon dynamic loading, although serrations are frequently observed on the stress-strain curve upon quasi-static compression at room temperature. Compared to the mechanical behaviors of BMGs upon dynamic loading, ex- and in-situ MGMCs are rendered to be good candidates for military and space applications, ascribed to their good self-sharpening property. 


\section{Fracture toughness of bulk metallic glasses and metallic glass matrix composites}

As an indispensible parameter for the application of structural materials, fracture toughness represents the resistance of materials to the propagation of cracks. The higher fracture toughness corresponding to the large energy absorption before fracture indicates the better damage tolerance. As promising engineering materials, the investigations on the fracture toughness of metallic glasses, especially the MGMCs, have been conducted in recent decades. This part will focus on the fracture-toughness behavior of BMGs and MGMCs.

\subsection{Fracture toughness of metallic glasses.}

Fracture toughness of BMGs under different conditions was investigated [362-364], and the values of fracture toughness are highly variable, spanning a wide range. Until now, the mechanism of fracture in metallic glasses have attributed to many extrinsic and intrinsic reasons. The challenge is to obtain metallic glasses with high toughness.

Due to the limit of the GFA of metallic glasses, the investigations on fracture toughness are very disperse and non-uniform in earlier studies. Indirect and non-standard methods are used to measure facture toughness in amorphous ribbons [365-370]. After 1990s, the advent of BMGs with a large size of even centimeters BMGs makes a giant leap for the structural application of metallic glasses, which also deepens the fundamental understanding of their fracture mechanisms. 
However, assessing the fracture toughness of metallic glasses is still full of challenges. The demanded specimen size for fracture toughness tests often exceeds the critical thickness for the GFA [371]. Up to now, the investigations of fracture toughness of BMGs are mainly centered on some BMGs with the large GFA, such as Zr-, Pd-, Cu-based BMGs, which makes it easier to utilize unified and conventional methods to measure their fracture toughness.

Gilbert et al. [372] reported that the monolithic $\mathrm{Zr}_{41.2} \mathrm{Ti}_{13.8} \mathrm{Cu}_{12.5} \mathrm{Ni}_{10} \mathrm{Be}_{22.5}$ (Vitreloy-1) BMGs possess a high precracked fracture toughness, about $55 \mathrm{MPa} \sqrt{\mathrm{m}}$, which is even comparable with that of the high-strength steels or aluminum alloys. Kawashima et al. [373] studied the toughness of $\mathrm{Zr}_{55} \mathrm{Al}_{10} \mathrm{Ni}_{5} \mathrm{Cu}_{30}$, revealing a precracked fracture toughness in a range of 35.9 - 50.3 $\mathrm{MPa} \sqrt{\mathrm{m}}$ with an average of 43.3 $\mathrm{MPa} \sqrt{ } \mathrm{m}$. A high precracked fracture toughness of $53 \mathrm{MPa} \sqrt{\mathrm{m}}$ was obtained in $\mathrm{Zr}_{50} \mathrm{Cu}_{40} \mathrm{Ni}_{10}$ [374]. In some other alloy systems, Gu et al. [375] studied fracture toughnesses of $\mathrm{Ti}_{40} \mathrm{Zr}_{25} \mathrm{Cu}_{12} \mathrm{Ni}_{3} \mathrm{Be}_{20}$ for both pre-cracked and notched specimens, which is in excess of $100 \mathrm{MPa} \sqrt{ }$, although they exhibit zero compressive plasticity at the same sizes, which is dramatically different from other brittle materials, such as ceramics, and silicon.

For some other BMGs, which are not adequately tough to produce the pre-cracks, notch toughness is also used to study the toughness of BMGs. Schroers et al. [376] showed that Pt-based BMGs $\left(\mathrm{Pt}_{57.5} \mathrm{Cu}_{14.7} \mathrm{Ni}_{5.3} \mathrm{P}_{22.5}\right)$ exhibited a high notch fracture toughness, approximately $80 \mathrm{MPa} \sqrt{ } \mathrm{m}$ and estimated a large notch-tip plastic zone of about $1.4 \mathrm{~mm}$ ahead of the notch radius of $200 \mu \mathrm{m}$. In $\mathrm{Cu}_{60} \mathrm{Zr}_{20} \mathrm{Hf}_{10} \mathrm{Ti}_{10}$, 
Wesseling et al. [377] reported notch-toughness values in excess of $65 \mathrm{MPa} \sqrt{\mathrm{m}}$ by using specimens with a root radius of $110 \mu \mathrm{m}$, and the estimated plastic zone size of $63.7 \mu \mathrm{m}$ is comparable to the order of magnitude of some Zr-based BMGs [373, 378].

However, although both precracked and notch toughness have been measured in many alloy systems, it should be noted that it's hard to compare them directly [379], because the notch fracture toughness shows an obvious correlation with the notch root radius $[374,380-383]$. Relative to the sharp crack tip of precracked specimens, the tip of the notch for notch specimens is blunt. In $\mathrm{Zr}_{44.1} \mathrm{Ti}_{12.6} \mathrm{Ni}_{6.8} \mathrm{Cu}_{13.4} \mathrm{Be}_{23.1}$, with the changes of the notch-root radii from $250 \mu \mathrm{m}$ to a fatigue precrack, the average toughness obtained increases from precracked specimens of $18.4 \pm 1.4 \mathrm{MPa} \sqrt{\mathrm{m}}$ to $131 \mathrm{MPa} \sqrt{\mathrm{m}}$ at the notch root radius of $250 \mu \mathrm{m}$ [383], indicating a significant effect of the notch radius. By simulating the mean-stress variation in the notch tips, Henann et al. [380] claimed that the fracture toughness increases linearly with the square root of the notch-tip radius.

It is also necessary to note here that some BMGs appear to be insensitive to the notch-radius variation. It is found that the root radius appeared to have insignificant influence on the facture toughness of the brittle $\mathrm{Mg}_{59.5} \mathrm{Cu}_{22.9} \mathrm{Ag}_{6.6} \mathrm{Gd}_{11} \mathrm{BMG}$ [384]. Unlike Zr-based glasses, which usually have a large plastic zone, a tiny plastic zone, only about $r_{\mathrm{p}}=5 \mu \mathrm{m}$, and a few shear bands can be observed ahead of the notch root of $150 \mu \mathrm{m}$, corresponding to the low fracture toughness, $\sim 10 \mathrm{MPa} \sqrt{\mathrm{m}}$. Similar insensitivity to the notch radius can also be noticed in $\mathrm{Ti}_{40} \mathrm{Zr}_{25} \mathrm{Cu}_{12} \mathrm{Ni}_{3} \mathrm{Be}_{20}$ [375] and $\mathrm{Zr}_{55} \mathrm{Al}_{10} \mathrm{Ni}_{5} \mathrm{Cu}_{30}$ [373]. Inappreciable differences in the fracture-toughness values can 
be seen for pre-cracked and notched specimens. Apart from the influence of different notch radii of specimens, an obvious inconsistency of fracture toughness in the BMGs with identical compositions and under uniform test procedures has been noticed in different studies. Even in the same systems, the fracture-toughness values vary greatly for each of the precracked or notched specimens [373]. It gives an implication that fracture toughness is very sensitive to the subtle fluctuation of the initial microstructure.

The improvement on GFA allows us to investigate the fracture toughness more systematically. However, almost all BMGs still show much poorer fracture toughness than other ductile metals [385], although they can potentially yield plastically at much higher strengths. It seems to be an inherent mutual contradiction between the properties of toughness and strength in materials. More recently, two kinds of BMGs combining remarkable toughness with high stresses have been reported in Pd[371] and Zr-based [386] alloy systems, showing excellent damage tolerance, which is previously inaccessible to the toughest and strongest engineering materials. Demetriou et al. [371] at Caltech reported that the $\mathrm{Pd}_{79} \mathrm{Ag}_{3.5} \mathrm{P}_{6} \mathrm{Si}_{9.5} \mathrm{Ge}_{2} \mathrm{BMG}$ exhibited a unparalleled combination of damage tolerance and high yielding stress, considering the lack of microstructural defects, such as dislocations for crystalline materials. More importantly, the results demonstrate that it is potentially accessible, remarkable toughness and high strength simultaneously in BMGs. As shown in Figure 50 [371], this BMG demonstrates an extensive plastic shielding of an initially sharp crack, accommodated by an extensive shear-band sliding. 
Compared with the expensive Pd-based BMGs, Xu et al. [386, 387]

successfully obtained a highly-tough $\mathrm{Zr}$-based $\mathrm{BMG}\left(\mathrm{Zr}_{61} \mathrm{Ti}_{2} \mathrm{Cu}_{25} \mathrm{Al}_{12}, \mathrm{ZT} 1\right)$ by the careful composition design, showing a high toughness comparable to the $\mathrm{Pd}_{79} \mathrm{Ag}_{3.5} \mathrm{P}_{6} \mathrm{Si}_{9.5} \mathrm{Ge}_{2} \mathrm{BMG}$ and a robust GFA (a critical diameter of $\sim 10 \mathrm{~mm}$ for a copper-casting method), as well as the absence of costly elements. Yi and Lewandowski et al. [364] also reported that a Zr-based BMG has high toughness at 77 $\mathrm{K}$, which stands out as an outstanding metallic alloy for engineering applications.

\subsection{Toughness mechanism in BMGs}

As mentioned above, the toughness of BMGs exhibits strong sensitivity to the subtle fluctuation of structural states and testing conditions. Many extrinsic effects have been considered to influence the fracture toughness, including the residual stress induced by the large thermal gradient of rapid quenching [388-390], test temperature [364], loading condition [374, 391], size effect [392], etc. For example, by comparing $\mathrm{Zr}_{55} \mathrm{Cu}_{30} \mathrm{Al}_{10} \mathrm{Ni}_{5}$ BMGs with different oxygen levels [393, 394], it is concluded that the oxygen trapped in oxides eases the initiation of a straight crack and embrittles the glass. It is noticed that it is not possible to obtain a precrack straight through the metallic glass in very low oxygen states. Residual stresses also play a significant role on fracture toughness. Aydiner et al. [395] simulated the residual stress in the Vit 1 plate with 8.25 $\mathrm{mm}$ in thickness and estimated the surface compression stress of - $230 \mathrm{MPa}$ and the interior tension stress of $+90 \mathrm{MPa}$. This non-uniform distribution of stress states in the BMG samples has been indicated to play significant influence on fracture toughness by 
lowering the stress intensity at the crack tip and by the superposition of residual stresses $[372,388,390]$.

In addition to these extrinsic factors mentioned above, the influence of internal microstructures of BMGs on a more subtle level has also been discussed in some studies concerning the free-volume effects. Although for the nominally-identical BMGs, the free-volume difference may still exist due to different processing conditions. In this regard, the capacity of generating multiple shear bands plays a very important role on the shear-banding behavior during the extension of the crack. Higher amounts of free volumes in BMGs promote more shear transformations and then stimulate the formation of macroscopic shear bands under the external force. When this flow process occurs at the crack, the stress concentration can be mitigated, resulting in higher toughness [371]. Launey et al. [388] confirmed that fracture toughness in $\mathrm{Zr}_{44} \mathrm{Ti}_{11} \mathrm{Ni}_{10} \mathrm{Cu}_{10} \mathrm{Be}_{25}$ is significantly degraded by the reduction of free volumes due to structural relaxation. Similar results are obtained in $\mathrm{Zr}_{61} \mathrm{Cu}_{17.3} \mathrm{Ni}_{12.8} \mathrm{Al}_{7.9} \mathrm{Sn}_{1}$ [396]. The modeling work by Falk et al. [397] and Rycroft et al. [398] pointed out that the volume involved in the shear transformation can affect the fracture toughness of metallic glasses.

In crystalline materials, the toughness is correlated with the competition between the plastic flow and brittle fracture. During deformation, the plastic deformation is considered to be controlled by the shear modulus, $\mu$, and the dilatation, and the bonding breakage corresponding to the brittle fracture near the crack is believed 
to be controlled by bulk modulus, Pugh et al. [399] introduced a solidity index, S, to characterize the toughness of materials.

$$
\mathrm{S}=\mu / \mathrm{B}
$$

It further demonstrated that a lower $\mathrm{S}$ favors a higher ductility and fracture toughness $[399,400]$. The same approach has also been taken on BMGs to describe shear banding in the plastic zone related to the shear transformations of cluster of atoms, dominated by $\mu$ and the dilatation of metallic glass, influenced by B in the vicinity of a crack tip [202, 401]. $\mathrm{S}$ is also expressed in term of Poisson's ratio, $v$, with a value of $3 / 2(1-2 v) /(1+v)$ for isotropic metallic glasses. Thus, the higher the solidity index, the lower is the Poisson's ratio.

Schroers and Johnson [376] attributed to the high fracture toughness of $\mathrm{Pt}_{57.5} \mathrm{Cu}_{14.7} \mathrm{Ni}_{5.3} \mathrm{P}_{22.5}(\sim 80 \mathrm{MPa} \sqrt{\mathrm{m}})$ to the lower $\mathrm{S}$. i.e., higher Poisson's ratio about 0.42. They thought that the low shear modulus was in favor of the formation of multiple shear bands to relax the stress by shear deformation, before the extensional instability occurs.

By the careful comparison of BMGs with different $v$ or S, Lewandowski et al. $[202,401]$ found that the fracture toughness of BMGs was closely related to the Poisson's ratio. If $v>0.31-0.32, \mathrm{BMGs}$ trend to be tough. Otherwise, they will show relatively low fracture toughness. This trend can be well explained that why the Pd- and Zr-based BMGs have a much higher fracture toughness than the Mg- and Fe-based BMGs. The finite-element simulation results using the Mohr-Coulomb-based constitutive model demonstrated that the extent of the plastic zone ahead of a notch tip 
and the excessive plastic strain with shear bands will be alleviated by a higher Poisson's ratio [402]. However, the insensitivity of Poisson ratio to the fracture toughness is reported in some Ni-based BMGs [403]. The fracture toughness of the Ni-based BMGs varies significantly, whereas they have small difference in Poisson's ratios.

Demetriou et al. [371] proposed that fracture toughness of BMGs is closely related to a dimensionless parameter, $f$, which represents the net activated shear-transformation events before a cavitation event in the core of an operating shear bands.

$$
\log f \sim T_{\mathrm{g}} / T(\mathrm{~B} / \mu-1)
$$

They attributed the very high fracture toughness in $\mathrm{Pd}_{79} \mathrm{Ag}_{3.5} \mathrm{P}_{6} \mathrm{Si}_{9.5} \mathrm{Ge}_{2}$ to both the larger $\mathrm{B} / \mu$ and $T_{\mathrm{g}}$. This law contains both the influence of glass transition and Poisson' ratio.

As discussed above, although a great success has been achieved to understand the tough mechanism in metallic glasses in term of various extrinsic and intrinsic factors. The above-mentioned exceptions in some alloy systems always remind us that the investigation on toughening mechanisms in BMGs is far from over. It appears that the high toughness can be related to the abundant shear-banding behavior near the crack tips. In this view, enhancing the free-volume concentration and reducing the structure relaxation will benefit the improvement of fracture toughness. A lower $\mu / \mathrm{B}$, or higher Poisson's ratio will also cause an easier shear transformation. However, it's still hard to obtain a composition, which has both the high GFA and adequate Poisson's ratio. The answers may lie in further understanding the toughening mechanisms of BMGs. 
Although many problems exist for the development of BMGs, it is a fact that BMGs possess super high toughness, as shown in the Ashby map in Figure 51 [404].

\subsection{Fracture toughness of MGMCs}

Owing to the lack of the sub-microstructure, metallic glasses are more sensitive to the flaws. Due to the absence of microstructures in monolithic BMGs, the plastic deformation occurs by the initiation and propagation of the local shear bands. As mentioned above, they possess poor ductility and relatively low fracture toughness, compared with crystalline materials. To circumvent this problem, MGMCs composites have been successfully obtained by introducing the ex or in-situ second phase. The presence of secondary phases can effectively arrest the highly-localized shear bands before they can develop and become cracks, and promote the formation of profuse shear bands. Consequently, significant improvement on toughness has been achieved in these MGMCs. Fracture toughness of these MGMCs relies on many factors, e.g., the composition, reinforcement size and fraction volume, and loading condition, etc., leading to various results. However, very few reports have dealt with the fracture toughness of MGMCs.

\subsubsection{Fracture toughness of ex-situ MGMCs}

In order to understand the effect of glass matrix on fracture toughness, two kinds of ex-situ composites with the same volume fraction of TiNb embedded in the Cu-based and Zr-based glass matrix, respectively, are fabricated [405]. Compared with the $\mathrm{Cu}$-based glass matrix, the higher toughness of the $\mathrm{Zr}$-based glass matrix is beneficial to the higher fracture toughness of the composite by promoting the formation 
of micro-crack and multiple shear bands at the crack tip. Thus, a tougher glass matrix will contribute to the whole fracture toughness of MGMCs.

Jang et al. [406] further considered that if the plastic zone of a glass matrix is larger than the average distance of particles, the shear bands can stably propagate in the glass matrix without causing the catastrophic failure. The plastic-zone size can be associated with the crack-tip opening displacement [407]. For a given volume fraction of the second phase, the average distance of particles will be constant. However, the higher fracture toughness of the glass matrix gives a larger plastic zone size. Therefore, the propagation of shear bands in the tougher glass matrix will extend more stably than in the relatively-brittle glass matrix.

Zheng et al. [408] stimulated the initiation and propagation of cracks in the fiber-reinforced BMG composite. Results indicate that the presence of fibers can slow down the crack propagation and significantly influence shear banding in the glass matrix, which enhance the fracture toughness of composites. Hassan et al. [409] further studied the effect of loading modes and temperatures on the fracture toughness of W-particulate-reinforced Zr-based MGMCs. They found that the toughness of MGMCs is higher than that of pure $\mathrm{W}$ and but lower than that of the Zr-based glass matrix. Increasing test temperatures leads to significant increases in fracture toughness under both Mode I and Mixed Mode I/II conditions.

Accordingly, the fracture toughness of MGMCs is influenced by many factors, including the intrinsic factors (compositions and microstructures, etc.) and extrinsic factors (loading temperatures, loading rates, etc.). As we know, MGMCs 
share the virtues of the high strength of the amorphous matrix and the large plasticity of the crystal phase. According to a Mode-I opening crack, the characteristic dimension, $R_{P}$, of a crack tip's 'plastic zone' can be expressed as:

$$
R_{p} \approx(1 / 2 \pi)\left(K_{I C} / \sigma_{Y}\right)^{2}
$$

where $\sigma_{\mathrm{Y}}$ is the applied nominal stress.

\subsubsection{Fracture toughness of in-situ MGMCs}

For in-situ MGMCs, microstructural length scales (such as length and scale of dendrites) should match the characteristic length to shield an opening crack tip. When a microstructural length scale of dendrites should match the microcrack-tip plastic zone, the stable plastic deformation occurs for the prohibition of the unconstrained propagation of the shear band. The shear bands are deflected between the glass matrix and the dendrite, similar to that in monolithic BMGs. The deflection of microcracks leads to the decrease of the crack-tip driving force, and, thus, bring about the mixed loading mode at the crack tip, which results in the high fracture toughness and large plasticity.

MGMCs containing nanocrystalline particles, such as nanoscale crystallites, can be produced by the annealing process from a metastable metallic glass or by controlling the solidification process. Ramamurty and co-workers investigated the effect of crystallization on the impact toughness through the annealing process in MGMCs of La-Al-Cu-Ni-Co [410] and Zr-Ti-Cu-Ni-Be-Al [411]. They found that the ductile to brittle transition was induced by the even rather low percentage of nanocrystals. For example, the impact toughness of the monolithic $\mathrm{La}-\mathrm{Al}-\mathrm{Cu}-\mathrm{Ni}-\mathrm{Co}$ 
BMG is $9.43 \mathrm{~kJ} / \mathrm{m}^{2}$. However, it decreased to $2.85 \mathrm{~kJ} / \mathrm{m}^{2}$ upon the partial crystallization (a volume fraction of $19 \%$ ). With increasing the crystallinity, the toughness containing a crystal volume fraction of $50 \%$ decreased to a minimum of $0.79 \mathrm{~kJ} / \mathrm{m}^{2}$. Then, with further increasing crystallinity over $80 \%$, toughness increased to $1.37 \mathrm{~kJ} / \mathrm{m}^{2}$. For the partially-crystallized composites with the composition of $\mathrm{Zr}-\mathrm{Ti}-\mathrm{Cu}-\mathrm{Ni}-\mathrm{Be}-\mathrm{Al}$, the impact toughness decreased for the formation of brittle intermetallic compounds by the annealing process. Although Poisson's ratio decreases with increasing the content of crystallization [202], the impact toughness reduces to near zero after the annealing process, which is unlike the empirical correlation between the fracture energy and Poisson's ratio for the monolithic BMGs.

For in-situ dendrite MGMCs, Hofmann et al. [94, 412] evaluated the fracture toughness and crack-resistance curve behavior. They found that the DH3 composite $\left(\mathrm{Zr}_{39.6} \mathrm{Ti}_{33.9} \mathrm{Nb}_{7.6} \mathrm{Cu}_{6.4} \mathrm{Be}_{12.5}\right)$ exhibited excellent fracture toughness, e.g., fracture toughness increased to $\sim 160 \mathrm{MPa} \mathrm{m}^{1 / 2}$. Obviously, the in-situ ductile dendrite-reinforced Zr-based MGMCs have the fracture toughness as high as those of the toughest steels and crystalline titanium alloys. Basically, these alloys have relatively low Young's modulus, giving rise to extremely-high values of $\mathrm{G}_{1 \mathrm{C}}(\sim$ $\left.\mathrm{K}_{1 \mathrm{C}}{ }^{2} / \mathrm{E}\right)$. It is reported that the fracture toughness of the Fe-based MGMCs with a $40 \%$ volume fraction of the dendrite is $40 \mathrm{MPa} \mathrm{m}^{1 / 2}$, which is higher than the value of 27 MPa $\mathrm{m}^{1 / 2}$ of the monolithic Fe-based BMG [413].

According to Figure 52 [412], it can be found that the fracture-toughness values of these composites depend on the fraction volume and spacing of dendrite 
phases. The dendrite volume fraction $(67 \%)$ and the dendrite spacing $(5 \mu \mathrm{m})$ of the DH3 alloy are higher than that of the DH1 composites (the volume fraction of $67 \%$ and the dendrite spacing of $2 \mu \mathrm{m}$ ), which shows that DH3 has the lower yielding strength that DH1 [94]. However, the toughness $\left(173 \mathrm{MPa} \mathrm{m}^{1 / 2}\right)$ and tension plasticity $(13.1 \%)$ of the DH3 alloy are higher than the values of DH1 (the toughness of $87 \mathrm{MPa} \mathrm{m}^{1 / 2}$ and tension plasticity of $9.58 \%$ ). Clearly, the Zr-based MGMCs have the 2 - 4 times tougher than their corresponding unreinforced matrix alloys of Vit $1\left(54 \mathrm{MPa} \mathrm{m}^{1 / 2}\right)$. As potential structural materials, in-situ MGMCs will undergo various temperatures during loading. Therefore, investigations concerning the effect of the temperature on the deformation behavior and fracture toughness are important. Roberts et al. [96] investigated the impact toughness of in-situ dendrites / MGMCs by Charpy impact from $300 \mathrm{~K}$ to $100 \mathrm{~K}$. They found that the toughness of the glassy matrix and Vit 1 was reduced to $\sim 30 \%$ of their room-temperature toughness. When the surrounding temperature was decreased to between $175 \mathrm{~K}$ and $200 \mathrm{~K}$, the impact toughness decreased rapidly. As the temperature is further deceased, the ductile-to-brittle transition behavior occurs in the dendrites. The impact toughness at $100 \mathrm{~K}$ is just $\sim 15$ $\%$ of the value at room temperature.

Hassan et al. [414] further investigated the effects of the testing temperatures from $150 \mathrm{~K}$ to $653 \mathrm{~K}$ and loading conditions on the fracture behavior and toughness using the precrack samples by bending tests. Under the same loading rates, the toughness is increased slightly below $505 \mathrm{~K}\left(0.77 T_{g}\right)$, and exhibits the same increasing trend with increasing temperature. As the temperature is increased to $603 \mathrm{~K}$, the 
toughness decreased to $20 \mathrm{MPa} \cdot \mathrm{m}^{1 / 2}$. On the other hand, under the same temperatures, the fracture toughness is decreased from $20 \mathrm{MPa} \cdot \mathrm{m}^{1 / 2}$ at $0.05 \mathrm{~mm} / \mathrm{s}$ to $15 \mathrm{MPa} \cdot \mathrm{m}^{1 / 2}$ at $0.5 \mathrm{~mm} / \mathrm{s}$ at $505 \mathrm{~K}$, which is similar to the results at $603 \mathrm{~K}$. However, at $623 \mathrm{~K}$, when the loading rate is increased from $0.5 \mathrm{~mm} / \mathrm{s}$ to $5 \mathrm{~mm} / \mathrm{s}$, the fracture toughness is increased from $5 \mathrm{MPa} \cdot \mathrm{m}^{1 / 2}$ to $20 \mathrm{MPa} \cdot \mathrm{m}^{1 / 2}$. The increased toughness in high-temperature ranges is caused by ductile fracture features of the dendrite phase and glass matrix, and the brittle fractures at high loading rates or low temperatures occur. For comparison, it is very interesting to note that an increased toughness is accompanied by lowering the surrounding temperatures for multicomponent CrMnFeCoNi high-entropy alloys (HEAs), which have exceptional damage tolerance with tensile strengths above $1 \mathrm{GPa}$ and fracture toughness values exceeding 200 $\mathrm{MPa} \cdot \mathrm{m}^{1 / 2}$ [415], as exhibited in Figure 51. 


\section{Fatigue behavior of metallic glass matrix composites}

According to the literature reports $[416,417]$, there are approximately $90 \%$ of all service failures due to mechanical problems, and $75 \%$ of ground vehicles failures are associated with fatigue. Therefore, fatigue is a very important property for structural applications and is necessary to be characterized. The fatigue lifetime is generally presented by the number of loading cycles to initiate and propagate a crack to a critical size, since fatigue failures of materials usually include three stages: crack initiation, stable crack propagation, and fast fracture [417]. However, fatigue behavior of BMGs and MGMCs is still quite limited, compared with the other mechanical property studies of BMGs and MGMCs [249, 304, 307, 418-420], which will be reviewed in this section.

\subsection{Fatigue behavior of BMGs}

The fatigue behavior of metallic glasses was mainly reported on ribbons or wires since 1975, due to the low GFA of BMG [421, 422]. Until 1998, the four-point-bending fatigue results of BMGs are first reported [423]. From then on, the fatigue behavior on different kinds of BMGs (e.g., Zr-, Fe-, and Cu-based BMGs) are studied extensively with various loading modes, such as three-point-bending, four-point-bending, and tension-tension modes [390, 424-435]. However, these fatigue results show large difference among BMG alloys, including fatigue limits and lifetime $[436,437]$. According to the reported studies, many factors can be involved to yield such a large difference of these fatigue results of BMGs, such as the composition, material quality, specimen geometry, chemical environment, surface condition, temperature, cyclic frequency, mean stress, and residual stress [436, 437].

Nevertheless, some factors might play a key role, while the others have minor effects on the fatigue properties of BMGs.

Bending tests are widely used to assess the fatigue resistance of engineering

materials due to the convenient specimen mounting and dismounting, and the uniform maximum tensile stress on the specimen makes it possible to test brittle materials in 
tension fatigue without special sample gripping, as illustrated in Refs. [437, 438] for the three- and four-point bending setups. The nominal maximum tensile stress $(\sigma)$ of the bending specimen can be calculated as follows:

$$
\sigma=\frac{3 P\left(\mathrm{~S}_{2}-\mathrm{S}_{1}\right)}{2 b t^{2}}
$$

where $P$ is the applied load, b is the specimen width, $t$ is the specimen thickness, $S_{1}$ is the inner span (zero for the three-point-bending setup), and $S_{2}$ is the outer span.

Since BMGs are brittle alloys, and their sizes are limited, so far, three- and four-point bending fatigue studies are employed widely. Gilbert et al. [423] first reported four-point-bending fatigue results of Vit1 BMG alloys. Later three- and four-point bending fatigue tests were used extensively to investigate the fatigue behavior of both BMGs and MGMCs.

The three- and four-point-bending fatigue behavior, represented by an S-N curve ( $\mathrm{S}$ is the applied stress, and $\mathrm{N}$ is the cycles to failure) of BMGs are shown in Figure 53(a) and (b) [437], respectively. It can be observed that the endurance limits of BMGs vary from 360 to $770 \mathrm{MPa}$ under three-point-bending fatigue [Figure 53(a)], while the endurance limits of BMGs ranged from 150 to $850 \mathrm{MPa}$ for the four-point-bending fatigue [Figure 53(b)]. Therefore, it is very clear that the fatigue properties (endurance limits and lifetimes) of BMGs display a wide range under bending-fatigue experiments.

\subsection{Fatigue behavior of MGMCs}

Besides monolithic BMG alloys, the fatigue behavior of MGMCs is also reported, mainly employing the four-point-bending fatigue test. Flores et al. [439] 
found that the fatigue-endurance limit and toughness of MGMCs with a composition of $\mathrm{Zr}_{56.2} \mathrm{Ti}_{13.8} \mathrm{Nb}_{5.0} \mathrm{Cu}_{6.9} \mathrm{Ni}_{5.6} \mathrm{Be}_{12.5}$ (developed based on the Vit1 alloy) was improved, compared with the Vit1 alloy, in which the composites contained a relatively-fine dispersion of dendritic crystalline inclusions. Qiao et al. [440] conducted four-point-bending fatigue tests on a nano-particle $(\sim 5 \mathrm{~nm})$-dispersed MGMCs with the composition of $\left(\mathrm{Zr}_{58} \mathrm{Ni}_{13.6} \mathrm{Cu}_{18} \mathrm{Al}_{10.4}\right)_{99} \mathrm{Nb}_{1}$, which have a fatigue-endurance limit of $559 \mathrm{MPa}$. Moreover, the four-point-bending fatigue of a dendritic inclusion ( $\beta$ phase) dispersed MGMC $\left(\mathrm{Zr}_{39.6} \mathrm{Ti}_{33.9} \mathrm{Nb}_{7.6} \mathrm{Cu}_{6.4} \mathrm{Be}_{12.5}\right.$, at.\%) was conducted by Launey et al. [441]. A relatively-high fatigue-endurance limit was obtained.

The stress-life (S-N) fatigue results of the MGMC $\left(\mathrm{Zr}_{39.6} \mathrm{Ti}_{33.9} \mathrm{Nb}_{7.6} \mathrm{Cu}_{6.4} \mathrm{Be}_{12.5}\right)$ are shown in Figure 54(a) [442]. The fatigue-endurance limit is $567 \mathrm{MPa}$, which is defined as the stress range with a lifetime larger than $10^{7}$ cycles, as shown in Figure 54. It can also be observed that the composites exhibit a decreasing fatigue-life cycle with increasing the stress range. The tension-tension fatigue result with the same composition is given as a comparison in Figure 54(a). It can be noticed that an increased fatigue limit is obtained for the four-point-bending fatigue, compared with the tension-tension fatigue for this MGMC [443]. Upon bending, half of the specimen is under compression loading, and the propagation of the fatigue-crack tip is not easy, while the crack can propagate easily under mode I upon tension loading. Therefore, the four-point-bending fatigue-endurance limit is improved, compared with that of tension-tension fatigue. For both tension-tension and four-point-bending fatigue, MGMCs have larger fatigue-endurance limits than 
$\mathrm{Zr}_{56.5} \mathrm{Cu}_{6.9} \mathrm{Ni}_{5.6} \mathrm{Ti}_{13.8} \mathrm{Nb}_{5.0} \mathrm{Be}_{12.5} \mathrm{MGMCs}$, since the composite

$\mathrm{Zr}_{58.5} \mathrm{Ti}_{14.3} \mathrm{Nb}_{5.2} \mathrm{Cu}_{6.1} \mathrm{Ni}_{4.9} \mathrm{Be}_{11.0}$ has a higher volume of crystalline dendrites [442, 443], which can effectively blunt the crack tip upon loading. For the

$\mathrm{Zr}_{39.6} \mathrm{Ti}_{33.9} \mathrm{Nb}_{7.6} \mathrm{Cu}_{6.4} \mathrm{Be}_{12.5} \mathrm{MGMC}$ [441], the characteristic length scales of the crack size and microstructure are matched correctly, which can work together to retard the initial extension of small flaws and to prevent the single shear-band opening failure.

Therefore, the $\mathrm{Zr}_{39.6} \mathrm{Ti}_{33.9} \mathrm{Nb}_{7.6} \mathrm{Cu}_{6.4} \mathrm{Be}_{12.5}$ MGMC exhibits a higher four-point-bending fatigue-endurance limit than other MGMCs. However, for the $\mathrm{Cu}_{47.5} \mathrm{Zr}_{38} \mathrm{Hf}_{9.5} \mathrm{Al}_{5}$ composite reinforced with a $\mathrm{CuZr}$ phase, the crystalline inclusion does not facilitate the retardance of the fatigue crack, which is attributed to the brittleness of crystalline second phases. Recently, the fatigue behavior of the $\mathrm{Zr}_{48} \mathrm{Cu}_{47.5} \mathrm{Co}_{0.5} \mathrm{Al}_{4}$ composite is investigated, and it is found that the fatigue endurance is much improved than the reported results on the MGMCs, as shown in Figure 54(b). It can be observed that the fatigue-endurance limit of $\mathrm{Zr}_{48} \mathrm{Cu}_{47.5} \mathrm{Co}_{0.5} \mathrm{Al}_{4}$ is $\sim 1,200 \mathrm{MPa}$, while in the reported results, the fatigue-endurance limit usually has a stress range of $200-700 \mathrm{MPa}$, which should be attributed to the stress-induced martensitic transformation of crystalline inclusions from $\mathrm{B} 2-\mathrm{CuZr}$ to $\mathrm{B} 19 '-\mathrm{ZrCu}[444]$.

The typical four-point-bending fatigue-fracture morphology of MGMCs is shown in Figure 55 [442] with the composition of $\mathrm{Zr}_{58.5} \mathrm{Ti}_{14.3} \mathrm{Nb}_{5.2} \mathrm{Cu}_{6.1} \mathrm{Ni}_{4.9} \mathrm{Be}_{11.0}$ at the stress range of $792 \mathrm{MPa}$ and cycle number of 14,776. Figure 55(a) displays the lateral surface near the fracture surface of MGMCs, in which no shear bands can be observed, indicative of a brittle failure of MGMCs, since the maximum applied stress is lower 
than the yielding strength. Four typical fatigue stages can be generally observed on the SEM fractograph: the crack-initiation site (stage I), the crack-growth region (stage II), the fast fracture region (stage III), and the final fracture region (stage IV). The fatigue-initiation site is exhibited in Figure 55(b), which is usually associated with casting defects. Under fatigue testing, stress concentration will appear around these defects, finally leading to an early failure of specimens. After initiation, cracks will propagate into stage II, as shown in Figure 55(c). Striations can be observed at this stage, which is formed by ductile deformation at the crack tip as it opens and closes. Along the propagating direction, striations form, and the strain energy dissipates. A magnified striation area is displayed in Figure 55(d), in which fine striations (hundreds of nanometers) can be observed beside the existence of coarse striations ( $\sim 3 \mathrm{~mm})$ both marked by arrows. The spacing of these fine striations is in accordance to the amount that the crack front moved forward during one stress cycle [443]. The stages II and III can be clearly separated by a dashed line, as displayed in Figure 55(e). Typical vein patterns dominate in the fast fracture region [stage III]. Figure 55(f) is a magnified graph of stage III, in which the final fracture region (stage IV) can also be noticed between the two dash lines with a spacing of $\sim 4 \mu \mathrm{m}$. The final fracture region is generally corresponding to a pure shear, similar to the shear-off set region during compression, accompanied with the light and sound emissions [427].

For the fractography of the composites subject to tension-tension fatigue at a stress range of $810 \mathrm{MPa}$, the four typical fatigue stages can also be observed, as shown in Figure 56 [443], including (I) the crack-initiation site, (II) the crack-growth 
region, (III) the fast fracture region, and (IV) the final fracture region. In Figure 56(a), the initiation site is located in the interior of the specimen. Stages II and III can be discerned by a dashed line. In Figure 56(b), a magnified area of stage II is exhibited near the dashed line, with a spacing of 4 to $6 \mu \mathrm{m}$ for coarse striations. Usually, there is a plastic zone ahead of the crack extension. When the crack extends into the glass matrix, the plastic strain is accommodated by the operation of shear bands. The crack easily extends along the shear bands, and the coarse striations form.

\subsection{Fatigue propagation of BMGs and MGMCs}

In general, the fatigue-crack-growth data of BMGs and MGMCs are shown in Figure 57(a) [436]. It can be observed that the fatigue-crack-growth rates of the Zr-based BMGs and composites are comparable to those observed in traditional crystalline alloys, such as an ultrahigh-strength steel (300-M) and an age-hardened aluminum alloy (2090-T81).

As proposed by Gilbert et al. [390], the crack-growth rate $(\mathrm{da} / \mathrm{dN})$ in a steady state of BMG and MGMCs follows the Paris law [445]:

$$
\frac{d a}{d N}=C(\Delta \mathrm{K})^{m}
$$

where $C$ is a material constant, $m$ is the crack-growth exponent, and $\Delta \mathrm{K}$ is the cycle-stress-intensity-range factor. $\Delta \mathrm{K}$ can be estimated from the morphology of the fracture surface [446], using the following equation:

$$
\Delta \mathrm{K}=\Delta \sigma \sqrt{\pi a} F(\lambda)
$$

where $a$ is the depth of the fatigue-crack tip, and $F(\lambda)$ is the boundary-correction factor and defined as follows (e.g., for the cylindrical sample): 


$$
F(\lambda)=0.66-0.02398 \lambda+1.664 \lambda^{2}
$$

here, $\lambda=\mathrm{a} / \mathrm{D}$, where $\mathrm{D}$ is the specimen diameter. The calculation at the final stage of stage II is very close to the experimental data of fine-striation spacings of 330 to 500 nm (Fig. 56). It is reasonable to deduce that each fine striation is created during one stress cycle for the present MGMCs on the basis of the analysis of experiments and calculations [446]. The decreasing crack-growth rate indicates the effective blunting of the ductile dendrites at the crack tip and the increased toughness of the composites [446]. As a consequence, the improved tension-fatigue limit is realized.

For Zr-based BMGs and composites, the crack-growth exponent is generally in the range of 1 to 3, which is typical of ductile crystalline alloys in the Paris regime [446]. However, the $\Delta \mathrm{K}_{\text {th }}$ values of $\mathrm{Zr}$-based BMGs are usually lower than those of crystalline alloys. The fatigue fracture toughness that starts the unstable fatigue-crack propagation in Zr-based BMGs is also much lower than in these crystalline alloys. This feature could be the reason why BMGs fail after a limited number of cycles once crack initiation has occurred.

The S-N curves of some typical crystalline alloys are also plotted in Figure 57(b) [437] to compare with the BMGs and MGMCs. Among the crystalline alloys, the ultrahigh-strength steel (300-M) with the strength of $2.3 \mathrm{GPa}$ displayed the highest endurance limit, followed by In 718 superalloy and Ti-6Al-4V, while the Zirconium alloys has the lowest endurance limit. The fatigue-endurance limits of BMGs and MGMCs are comparable with these conventional crystalline materials, but depending 
on the experimental methods on the BMG and MGMC alloys, especially the alloy composition modification and fabrication procedure.

An examination of these plots (Figures 53 and 57) demonstrates that the fatigue life-time of BMGs and MGMCs are comparable under three- and four-point-bending fatigue. However, the test volume under three-point bending is much smaller than that under four-point bending. In general, a greater test volume usually includes more defects and free volumes in BMG or MGMCs, which will facilitate the shear-band formation and crack initiation, therefore, leading to lower fatigue endurance limits in four-point-bending than in three-point-bending tests.

Moreover, a shear-band mechanism is proposed by Liaw et al. to explain the characteristics of the observed fatigue cracking [447]. It can be commonly noticed that there are two kinds of striations, fine and coarse striations [447], observed in the crack-propagation region for BMGs. The fine striation is related to the crack propagation by one fatigue cycle, while the coarse striations are caused by the discontinuous shear bands ahead of the fatigue crack. In crystalline alloys, the fatigue-crack-growth mechanism is related to a specified movement of dislocations, while shear banding plays a critical role in the fatigue behavior of BMGs [257].

During fatigue loading, shear bands usually form near flaws, defects, or machining marks in BMGs, which easily leads to stress concentrations. In general, the material density is low within shear bands, including many flow defects, in the form of free volumes. Thus, shear-band areas are easier to be deformed in BMGs. It can be imagined that a fatigue crack initiates from the opened shear band, and then 
preferentially propagate along the shear bands. During fatigue loading, the fatigue crack will advance in a direction perpendicular to the tensile-stress direction after each fatigue cycle. At the crack tip, a plastic zone is formed in which many shear bands appear. A plastic wake is produced due to the plastic zone left by the propagating crack, which is schematically shown in Fig. 57(c)(i) [447]. Then, the fatigue crack prefers to propagate along the shear bands at the crack tip, since shear bands are weaker than the bulk. As displayed in Fig. 57(c)(ii) [447], the crack advances one fine striation spacing every fatigue cycle. In general, the crack may propagate for several cycles along one shear band to form the coarse striation, as illustrated in Fig. 57(c)(iii) [447]. With increasing the crack length, the maximum stress intensity, $\mathrm{K}_{\max }$, becomes larger and larger. When $\mathrm{K}_{\max }$ reaches the fracture toughness, $\mathrm{K}_{\mathrm{Ic}}$, the BMG specimens fail.

\subsection{Simulations on fatigue behavior of MGMCs}

According to Wang's work [448], the fatigue characteristics and mechanisms of BMGs and MGMCs can be understood through the molecular-dynamics (MD) simulation, especially the stress-driven disorder-to-order transitions [259, 449], although it is not studied as extensively as the temperature-driven structural disorder-to-order transition process [450-452]. Irrespective of the stimulation by temperature or stress, the structural transition from the disorder to order states requires "diffusive" reshuffling of atoms. Although it is well known that STZs promote the plastic deformation of BMGs under stress, how the stress leads to "atomic diffusivity" in BMGs is still not quite clear. To quantify and understand the atomic-structure evolution, the MD simulations of the cyclic-stress-induced glass-to-crystal transition 
were reported on a binary $\mathrm{MG} \mathrm{Al}_{50} \mathrm{Fe}_{50}$ alloy. The details of $\mathrm{MD}$ simulations can be found in Ref. [448]. The evolution of atomic geometries is analyzed by the "deformation-diffusion" decomposition,

$$
d_{j i}=d_{j i}^{0} J_{i}+s_{j i}, j \in N_{i}
$$

where $\mathrm{i}$ is the analyzed atom, $\mathrm{j} \in \mathrm{N}_{\mathrm{i}}$ are $\mathrm{i}$ 's original neighbors in a reference configuration, $d_{j i}^{0}$ is the original distance between atoms, $\mathrm{j}$ and $\mathrm{i}$, in the reference configuration, and $d_{j i}$ is their current distance vector. The first term in Eq. (44) reflects the local deformation, while the second term reflects additional atomic movements beyond mere shape change. The extent of local diffusion is defined by

$$
D_{i}^{2}=\frac{1}{N_{i}} \sum_{j \in N_{i}}\left|\mathrm{~s}_{j i}\right|^{2}=\frac{1}{N_{i}} \min _{J_{i}} \sum_{j \in N_{i}}\left|d_{j i}^{0} \mathrm{~J}_{i}-d_{j i}\right|^{2}
$$

where the local deformation gradient, $\mathrm{J}_{\mathrm{i}}$, needs to be numerically optimized to minimize $D_{i}^{2}$, which will accumulate during repeated cyclic mechanical deformation. Figure 58(a) shows the atomic configuration of the notch area after 275 cycles of fatigue loading, in which the long-range atomic arrangement represents crystallization [448]. Statistics of the atomistic-diffusional displacement $\left(D_{i}^{2}\right)$ after different cycles $(1,20$, 70, 175, and 275) are plotted in Figure 58(c). It can be noticed that the variation of atom fraction vs. $D_{i}^{2}$ in the first half cycle showed a sharp decrease, followed by an increased atom fraction and reaching a peak value at large $D_{i}^{2}$ with increasing the cycle number, e.g., the corresponding $D_{i}^{2}$ equals $5.8 \AA^{2}$ for the peak after 275 cycles. Although the peak $D_{i}^{2}$ becomes sharper with increasing numbers of cycles, it occurs at an approximately constant of $D_{C}^{2}\left(\sim 5.5 \AA^{2}\right)$, which represents the initiation of crystallization. Moreover, the averaged $\mathrm{D}^{2}$ showed a nonlinear increase with 
increasing strain cycles, as the black solid line shown in Figure 58(d), in which the average grain size can be classified into three regimes according to the cycle number [Figure 58(d), black circle curve]. In regime I (cycles from 1 to 110), the average grain size remains a subnanometer. In regime II (cycles from 110 to 175), rapid grain growth starts following the incubation period (regime I). In regime III (cycles after 175), the grain-growth rate decreases significantly, but the $\mathrm{D}^{2}$ still increase. Atomistic simulations of monotonic deformation (strain $8 \%$ ) of the same sample geometry are shown in Figure 58(b) to compare with cyclic deformation [Figure 58(a)]. It can be observed that neither the obvious localization nor long-period atomic arrangement of atoms appears. To quantify the relative diffusive to displacive nature of the structural transformation based on atomic trajectories, a “diffusion-to-shear ratio," $\bar{D}^{2} / \bar{J}_{2}$, is calculated, where $\bar{J}_{2}$ is the mean Mises shear strain invariant from $\mathrm{J}_{\mathrm{i}}$. From the MD simulations, monotonic loading beyond global yielding gives a lower $\bar{D}^{2} / \bar{J}_{2}$ than subyielding cyclic loading. Moreover, the value of $\bar{D}^{2} / \bar{J}_{2}$ increases with incremental deformation cycles, due to the cumulative nature of random atomic displacements. Therefore, crystallization is easier to appear in cyclic loading than monotonic loading. Note that crystallization in both experiments and simulations is a mechanical deformation-driven process without the obvious temperature rise. 


\section{Wear and corrosion resistance of metallic glass matrix composites}

\subsection{Wear behavior of bulk metallic glasses and their composites}

Metallic glasses have excellent mechanical, physical, and chemical properties owing to their unique structures. These outstanding features have attracted much attention as well as wide promising applications in many fields. BMGs are supposed to have excellent anti-abrasive behavior because of their high strength and hardness. However, the results from recent studies seem to have a conflict with this trend. Some research reveals that metallic glasses perform better during the abrasive test than their crystalline counterparts, or even better than the traditional structural materials [453, 454]. However, some other research points out that metallic glasses behave much worse than expected wear resistance during the friction tests $[455,456]$. Such conflict may be explained in this way: the friction and wear behavior is not the intrinsic properties for materials, but the performances under certain conditions. They are in large correlation with abrasive conditions and testing parameters, such as loading, sliding speed, sliding distance, lubricating condition, friction mode, testing environment, and so on.

(1) The effect of loading

Loading is an important parameter during friction. Fu et al. [457, 458] studied the tribological behavior of the $\mathrm{Zr}_{41.2} \mathrm{Ti}_{13.8} \mathrm{Cu}_{12.5} \mathrm{Ni}_{10} \mathrm{Be}_{22.5} \mathrm{BMG}$ under different loads. They found that with increasing the load, the coefficient of friction declined, while the wear rate rose. And such phenomena existed in different abrasive counterparts and 
testing conditions, as shown in Figure 59(a) and (b) [457]. Similar behavior was also observed in the $\mathrm{Fe}_{40} \mathrm{Ni}_{40} \mathrm{~B}_{20} \mathrm{BMG}$ [459-461] and the friction between the Co-based metallic glass and alloy steels [462]. Wong and Li [463] have found that the wear loss of the as-cast and as-quenched $\mathrm{Fe}_{81} \mathrm{~B}_{13.5} \mathrm{Si}_{3.5} \mathrm{C}_{2}$ BMGs increased linearly with increasing load.

(2) The effect of the sliding speed

The sliding speed, a crucial parameter during friction, has an interesting relationship with abrasive and friction coefficients. The correlation is not linear, but behaves like a single curve with a summit $[457,462]$. The coefficient of friction for the $\mathrm{Zr}_{41.2} \mathrm{Ti}_{13.8} \mathrm{Cu}_{12.5} \mathrm{Ni}_{10} \mathrm{Be}_{22.5} \mathrm{BMG}$ decreases, as the speed increases and reaches the minimum rate at $1 \mathrm{~m} / \mathrm{s}$. When the speed goes higher, the heat generated during sliding will interfere with the measurement [457], as shown in Figure 59(c). Li and Wang [462] studied the correlation between the sliding speed and frictional coefficient on two Co-based metallic glasses. The results revealed that there was a maximum value for the frictional coefficients at different sliding speeds. Possible explanations may be due to the transformation of abrasive mechanisms, which was caused by the oxidation of metallic glasses during friction.

(3) The effect of the sliding distance

Theoretically, the wear loss of materials will increase with increasing the sliding distance. However, from a practical view, this trend is not always the case. This is because an adaption exists in the primary friction stage. The wear loss during this stage can be either more severe or less severe than the stable friction stage [464]. Figure 
60 presents the effect of the wear volume and wear rate on the sliding distance on four BMGs, as shown in (a) [465] and (b) [466], respectively. A linear relationship is present, if the primary stage is ignored [465]. The $\mathrm{Zr}_{41.2} \mathrm{Ti}_{13.8} \mathrm{Cu}_{12.5} \mathrm{Ni}_{10} \mathrm{Be}_{22.5} \mathrm{BMG}$ possesses a severe volume loss during early stages of sliding [466], as shown in Figure 60(b). Similar behavior was observed by Ozawa et al. [467]. Wong and Li [463] found that the wear loss of $\mathrm{Fe}_{81} \mathrm{~B}_{13.5} \mathrm{Si}_{3.5} \mathrm{C}_{2}$ BMGs was minimal that it could be ignored during the primary adapted stage.

(4) The effect of the lubricating condition and friction mode

Blau [468] pointed out that the wear resistance of the $\mathrm{Zr}_{52.5} \mathrm{Cu}_{17.9} \mathrm{Ni}_{14.6} \mathrm{Al}_{10} \mathrm{Ti}_{5}$ BMG was worse under lubricating conditions. Similar results were also reported in Bakka's work [469]. Ishida et al. [470] performed sliding-wear and rolling-wear tests on the $\mathrm{Ni}_{53} \mathrm{Nb}_{20} \mathrm{Ti}_{10} \mathrm{Zr}_{8} \mathrm{Co}_{6} \mathrm{Cu}_{3} \mathrm{BMG}$ and found that the latter has a great wear resistance than the former. Thus, the tribological behavior of BMGs is closely related to the friction mode. Fleury et al. [471] reported the different wear behavior of a series of BMGs under the reciprocating and unidirectional motion.

(5) The effect of the environment

Fundamental understanding is still needed on the wear behavior of metallic glasses under different environments. Despite the fact that several reports have claimed the sensitivity of metallic glasses to wear environments, the detailed mechanisms are still unclear. Miyoshi and Buckley [472] studied the wear properties of Fe-based metallic glass by pin-on-disc devices. They pointed out that the oxide layer formed on the surface of metallic glass is very stable. Such layer can not only reduce 
the coefficient of friction, but also prevent the substrate from abrasion. Wu et al. [473, 474] studied the effects of oxygen on the tribological behavior of a Zr-based BMG using pin-on-disk wear tests in three different environments, i.e., air, oxygen, and argon. It was found that the wear rate of the BMG specimens increased dramatically with increasing the oxygen content in the testing environment. Figure 61(a) and (b) revealed that the outermost-worn surface consisted mainly of monoclinic and tetragonal $\mathrm{ZrO}_{2}$ [473]. By comparing the abrasive behavior of BMGs between air and vacuum environments, Fu et al. [457] found that the wear loss was less in vacuum, and yet the coefficient of friction was larger.

(6) The effect of the friction counterpart

So far, the reports on the friction counterpart are relatively rare, and the results are contradictory. Fu et al. [457] discussed the tribological behavior between the Zr-based BMG and itself or 52100 steel. It was shown that the change in the counterpart does not significantly affect the coefficient of friction or wear loss. An interpretation for such a phenomenon can be put in this way: During the friction between the metallic glass and 52100 steel, a transfer of matters will happen from the metallic glass to the counterpart of steels, and, thus, lead to the formation of a metallic glass layer on the steel. Fleury et al. [471] pointed out that the change on the surface of the metallic glass during friction can depend on either its friction counterpart or the friction mode. Wu et al. [475] used pin-on-disk sliding measurements to study the tribological behavior of a $\mathrm{Zr}_{52.5} \mathrm{Cu}_{17.9} \mathrm{Ni}_{14.6} \mathrm{Ti}_{5} \mathrm{Al}_{10} \mathrm{BMG}$ against the zirconia and steel as counterparts. The results showed that the wear of the Zr-based BMG pins against a steel counterpart was 
considerably larger than against a zirconia. The viscous flow and transferred materials induced by frictional heating took place on the worn surface of the glassy pin and the counterpart disk, respectively.

\subsection{The wear mechanisms}

Under the friction force, the abrasive surface will suffer from a shear stress along the sliding direction, leading to its break-down. Thus, the metal debris is peeled off from the surfaces. If the difference of the hardness values between two friction counterparts is large, metals will transfer from the soft surface to the hard one, leading to the adhesive wear. If the hardness values of two friction counterparts are equal or similar, debris in slice or particles formed will finally peel from two surfaces, leading to the stripping wear or abrasive wear. The peel of debris will inevitably lead to the initiation and expansion of cracks. Based on this asperity-contact model, Archard [476] puts forward an abrasive equation:

$$
\tilde{W}=k_{A} \tilde{F}
$$

where $k_{\mathrm{A}}$, named as Archard Coefficient, is a parameter related to the material and the abrasive counterpart.

(1) Adhesive wear

Adhesive wear occurs when surfaces slide against each other, and the pressure between the contacting asperities is high enough to cause the local plastic deformation. During deformation, the matter transformed from soft to hard surfaces, leaving a scratch (also called furrows) on the soft surface. Thus, the worn surface produced by the adhesive wear is normally characterized by the transfer of materials 
from one surface to another. The wear equation of the adhesive wear is consistent with Eq. 46, and the $k_{\mathrm{A}}$ value ranges from $10^{-3}$ to $10^{-5}$ [464]. Parlar et al. [454] investigated the sliding tribological properties of the $\mathrm{Zr}$-based BMG. The analysis on the wear track from the worn surface and collected wear debris indicated that the BMG surface experienced the severe plastic deformation with the inhomogeneous shear deformation, abrasive and adhesive wear during sliding. The decrease of wear rates is related to the material transfer from the counterpart and the formation of protective oxidation during sliding.

(2) Abrasive wear

Abrasive grains, the particles with the high hardness, sharp corners, and ridges exist at the interface abrasive surface. They can directly cut asperities into debris, or plunge into the counter friction surfaces and then shape into debris by deformation. The wear equation of the abrasive wear is also consistent with Eq. (46), but the $k_{\mathrm{A}}$ value varies from $10^{-1}$ to $10^{-2}$ [464], and the wear loss is higher than the one of adhesive wear. Siegrist et al. [477] investigated the tribological properties of the $\mathrm{Zr}_{52.5} \mathrm{Cu}_{17.9} \mathrm{Ni}_{14.6} \mathrm{Al}_{10} \mathrm{Ti}_{5} \mathrm{BMG}$. No microcracks were found in the complete amorphous alloy abrasive zone, and the abrasive mechanism is the micro-plowing effect in the supercooled liquid region. They also investigated the graphite and $\mathrm{ZrC}$ / MGMCs. The effect of the metallic glass and its composites on the friction coefficient can be divided into two regions, a low-coefficient and a high-coefficient regions. The former is only one third of the latter in the numerical value. They believed that this variation 
can be attributed to the contamination of the matrix material, which further resulted in the geometrical change of the friction track.

(3) Stripping Wear

Stripping wear is due to the accumulation of the stress-induced plastic deformation during the contact and interaction of asperities. The accumulation of plastic deformation will lead to periodic dislocations under the surface of metals. Under the plastic sheer stress, the accumulation of stress deformation causes a pile-up of dislocations around inclusions at a certain depth of the sub-surface, then leading to the formation of cracks or holes. According to the stress-field theory, when cracks initiate at a certain depth, a normal stress paralleled to the surface will prevent the expansion of cracks from the direction vertical to the surface. So the cracks are forced to expand along the direction parallel to the surface at a certain depth. When the cracks reach the critical length, the materials between cracks, and the material surface will be peeled off as debris.

The wear rate of the stripping wear is consistent with the Archard wear equation $[477,478]$, and

$$
k_{A}=2 \gamma_{0} f_{V} / f_{A}^{\prime}
$$

where the coefficient, $k_{\mathrm{A}}$, is related to the plastic shear strain, $\gamma_{0}$, the volume fraction of inclusions, $f_{v}$, the volume fraction of the critical hole area, when leading the debris to rapidly fracture, $f_{A}^{\prime}$, and $k_{\mathrm{A}}$ varies from $10^{-3}$ to $10^{-5}[477,478]$

Few reports are on the wear behavior of MGMCs [477, 479]. It was found that adding a low volume content of graphite, and especially $\mathrm{ZrC}$ generates a significant 
decrease in the coefficient of friction. The investigation of the wear rate indicates that the graphite- / ZrC-reinforced bulk metallic glass composites display an even lower wear rate than the 100Cr6-bearing steel [477] .

Figure 62 shows the variation in the friction coefficient of in-situ $\mathrm{Zr}_{58.5} \mathrm{Ti}_{14.3} \mathrm{Nb}_{5.2} \mathrm{Cu}_{6.1} \mathrm{Ni}_{4.9} \mathrm{Be}_{11.0}$ MGMCs with the sliding time under different normal loads and sliding velocities [479]. It can be clearly seen that the friction coefficient is reduced fast from a higher initial value of about 0.443 to 0.299 during the first $75 \mathrm{~s}$ under the lower loading and sliding velocity due to the settling down of the indenter and the breaking-in of the worn surface for MGMCs to that of the steel counterpart. The initial coarse surface was smoothened owing to the higher friction during the running-in period. Then the friction coefficient fast increases up to a steady state. However, the curves are quite unstable with great many waves caused by the rapid damage of the worn surface. The friction coefficient becomes steadier with the values varying between 0.299 and 0.415 . At a higher normal load of $10 \mathrm{~N}$, the friction coefficient slightly increases within the first $160 \mathrm{~s}$, and then the trend almost exhibits a steady state due to the formation of lubricating films with increasing the normal load. Furthermore, the values of friction coefficients always show a very steady status with the range of $0.30-0.43$ at $0.3 \mathrm{~m} / \mathrm{s}$ attributed to more contact areas between the rubbing surfaces and lubricating films. Overall, the friction coefficient shrinks in the range of $0.27-0.38$ at $10 \mathrm{~N}$. In addition, the curve of the friction coefficient fluctuates with numerous waves in the large amplitude until $290 \mathrm{~s}$ at $0.4 \mathrm{~m} / \mathrm{s}$. Then the trend fluctuates almost about a constant of 0.33 , indicating that the running-in period was longer with 
an increase in the sliding velocity. The friction coefficients are between 0.235 and 0.400. Besides, during contact wear, a high temperature rise may lead to crystallization, which affects the wear behavior [480]. As a whole, little attention has so far been paid to the tribological properties of ex-situ and in-situ MGMCs. More studies should be focused on the wear behavior of MGMCs.

\subsection{Corrosion resistance of bulk metallic glasses and their composites}

With the ever increasing number of the new BMGs systems and very recent fast-growing understanding of the mechanisms regarding their excellent mechanical properties, the application prospect of these amorphous alloys as practical engineering materials has been more promising. Subjected to the actual service surrounding, corrosion is inevitable. Therefore, the study of corrosion resistance of BMGs and MGMCs is essential.

Previously, the corrosion resistance of BMGs is widely investigated. For example, Pang et al. [481] synthesized Fe-Cr-Mo-C-B-P BMGs with the high corrosion resistance in aggressive $\mathrm{HCl}$ solutions. The BMGs maintained the metallic luster even by anodic polarization or after immersion for 1 week in an extremely aggressive $12 \mathrm{~N} \mathrm{HCl}$ solution. This trend is presumably due to the synergistic effect of molybdenum and chromium in the single solid-solution phase formed. The metalloid element, $\mathrm{P}$, beneficially affects both the kinetics of passivation and the composition of passive films, which lead to the improvement of corrosion resistance of BMGs. Later, Guo et al. developed a novel $\mathrm{Fe}_{43} \mathrm{Co}_{4.5} \mathrm{Cr}_{16.5} \mathrm{Mo}_{16.5} \mathrm{C}_{14} \mathrm{~B}_{4} \mathrm{Y}_{1.5} \mathrm{BMG}$, which possesses the 
corrosion resistance comparable to (or better than) that of a 316L stainless steel in a simulated acid rain and seawater solution [482]. Wang et al. [483] increased the corrosion resistance of Fe-based BMGs by minor alloying.

Besides, the corrosion resistance of Zr-based BMGs is extensively studied [484-486]. Li et al. [485] found $\mathrm{Zr}_{56} \mathrm{Ni}_{20} \mathrm{Al}_{15} \mathrm{Nb}_{4} \mathrm{Cu}_{5}$ BMG with excellent corrosion resistance in $\mathrm{H}_{2} \mathrm{SO}_{4}, \mathrm{HCl}$, and $\mathrm{NaCl}$ solutions. In-situ anodic-polarization experiments revealed a tendency towards a diffusion-controlled mechanism other than passivity in $\mathrm{Zr}_{50} \mathrm{Cu}_{40} \mathrm{Al}_{10}$ BMGs [483]. Jiang et al. [486] has investigated the corrosion behavior of the as-cast, deformed, and relaxed $\mathrm{Zr}_{52.5} \mathrm{Cu}_{17.9} \mathrm{Ni}_{14.6} \mathrm{Al}_{10.0} \mathrm{Ti}_{5.0}$ BMGs using cyclic-anodic-polarization tests. It is speculated that the corrosion behavior is related to the amount of free volumes. As the free volume decreased, the glasses became more corrosion resistant [486]. The as-cast and the deformed glasses are less resistant to pit propagation, while the relaxed one is more vulnerable to pit nucleation. The increase in free volumes favors the pit propagation, and the chemical ordering caused by the relaxation may promote the pit nucleation.

At present, there is little information about the corrosion resistance of MGMCs. The investigation of the corrosion resistance can't only explore the corrosion mechanism but expand practical applications of MGMCs. For instance, by coating metallic glass thin films on the steel wires, the corrosion resistance is considerably enhanced, ascribing to strong protective layers grown on the surface of the coated steel wires by anodization [487]. Gu et al. [488] found that $\mathrm{Cu}_{47.5} \mathrm{Zr}_{47.5} \mathrm{Al}_{5} \mathrm{MGMCs}$, including $\mathrm{CuZr}$ nanocrystals, exhibits the greater corrosion potential and lower 
corrosion current density as well as the distinct passive region. Combined with the corrosion morphologies, it uncovers that MGMCs have a higher corrosion resistance in the seawater than that in monolithic BMGs, which is attributed to an appropriate amount of $\mathrm{CuZr}$ nanocrystals dispersed homogeneously in the glassy matrix.

The chemical and electrochemical corrosion properties of the in-situ $\mathrm{Ti}_{45}(\mathrm{Zr}-\mathrm{Be}-\mathrm{Cu}-\mathrm{Ni})_{55-\mathrm{x}} \mathrm{Nb}_{\mathrm{x}} \mathrm{MGMCs}$, with $\mathrm{x}=0,5,10$, and 15 at. $\%$ and containing different volume fractions of crystalline $\beta-(\mathrm{Ti}, \mathrm{Zr}, \mathrm{Nb})$ phases, have been investigated in a hydrogenated $1 \mathrm{M} \mathrm{H}_{2} \mathrm{SO}_{4}+2 \mathrm{ppm} \mathrm{F}^{-}$electrolyte at $353 \mathrm{~K}$ [489]. In comparison to the monolithic Ti-based BMGs and Ti-6Al-4V alloy, the in-situ composite with the 20 vol. $\%$ of $\beta-(\mathrm{Ti}, \mathrm{Zr}, \mathrm{Nb})$ phases exhibits high corrosion resistance. Furthermore, the results indicate that the corrosion properties of the Ti-based BMGs are enhanced, as the $\mathrm{Nb}$ content is increased, and the in-situ MGMCs formed with 10 at. $\% \mathrm{Nb}$ exhibit the best corrosion resistance. However, as the volume fraction of dendrites is increased to $40 \%$, as a result of the increase of the $\mathrm{Nb}$ content, the corrosion properties are found to be moderately degraded. This trend was explained by the difference in the $\mathrm{Nb}$ contents between the matrix and the dendrites. In composites with a volume fraction larger than $21 \%$, (i.e., $\mathrm{Nb}=15$ at. $\%$ ), most of $\mathrm{Nb}$ atoms are used to form the dendrites. That excessive difference in the compositions between the dendrite and matrix induces a galvanic effect, which results in the dissolution of the less corrosion-resistant phase, i.e., the matrix in these in-situ composites. After immersion tests, the crystalline dendrites emerge from the amorphous matrices, suggesting that the matrices have been preferentially dissolved. Results of Auger electron spectroscopy (AES) analyses after 
potentiostatic tests indicate that the oxide layer formed on the matrix is essentially composed of $\mathrm{TiO}_{2}$, and the oxide layer formed on the surface of the dendrites is found to include both $\mathrm{TiO}_{2}$ and $\mathrm{NbO}$ oxides. The combination of these two kinds of oxides is believed to provide a high corrosion resistance to the dendrites.

In our research group, the corrosion behavior of in-situ dendrite / metallic glass matrix composites has been investigated very recently [490-492]. In order to understand the corrosion resistance of these in-situ $\mathrm{Ti}_{40} \mathrm{Zr}_{24} \mathrm{~V}_{12} \mathrm{Cu}_{5} \mathrm{Be}_{19} \mathrm{MGMCs}$, three typical environments have been chosen for electrochemical tests, which are 10 vol.\% $\mathrm{H}_{2} \mathrm{SO}_{4}, 40$ wt. $\% \mathrm{NaOH}$, and 3.5 wt. $\% \mathrm{NaCl}$ aqueous solutions, respectively [490]. Potentiodynamic polarization and electrochemical impedance spectroscopy (EIS) were conducted on these samples. Figure 63 shows the typical polarization curves of in-situ dendrite-reinforced $\mathrm{Ti}_{40} \mathrm{Zr}_{24} \mathrm{~V}_{12} \mathrm{Cu}_{5} \mathrm{Be}_{19}$ MGMCs in different solutions [490]. The corrosion current density, $\mathrm{I}_{\text {corr }}$, for the sample in $\mathrm{NaOH}$ solutions is significantly larger than its counterparts in both $\mathrm{H}_{2} \mathrm{SO}_{4}$ and $\mathrm{NaCl}$ solutions. Also notably, the polarization profile of the sample in $\mathrm{NaCl}$ solutions is rather different from the other two in both $\mathrm{H}_{2} \mathrm{SO}_{4}$ and $\mathrm{NaOH}$ solutions [493], which are characterized with a wide passivation region. Apparently, it suggests that the in-situ dendrite-reinforced $\mathrm{Ti}_{40} \mathrm{Zr}_{24} \mathrm{~V}_{12} \mathrm{Cu}_{5} \mathrm{Be}_{19}$ MGMCs have an impressive corrosion resistance in the strong acidic environment, while their performance suffered badly in the strong alkaline environment. In other words, their passivation layer in $\mathrm{NaOH}$ was not complete or stable. The irregular passive region of polarization curves from the sample in the $\mathrm{NaCl}$ solution was attributed to the presence of the attacking elements, $\mathrm{Cl}^{-}$ion, which can be adsorbed 
quickly on passive films at the beginning of the corrosion process and does damage on films subsequently.

The excellent corrosion resistance in the acid environment can be attributed to the formation of a passive film on the sample surface. Figure 64 represents the X-ray photo-electronic spectroscopy (XPS) depth profiles of states and distributions of alloying elements in the $\mathrm{Ti}_{46} \mathrm{Zr}_{20} \mathrm{~V}_{12} \mathrm{Cu}_{5} \mathrm{Be}_{17}$ passive film after the elechemical corrosion experiment in the $10 \mathrm{vol} . \% \mathrm{H}_{2} \mathrm{SO}_{4}$ solution [491]. It was indicated that the main element, $\mathrm{Zr}$, of passive films is composed of two kinds of states: $\mathrm{Zr}^{4+}$ and $\mathrm{Zr}^{0}$. Ti was also found by the XPS analysis in the form of $\mathrm{Ti}^{4+}$ and $\mathrm{Ti}^{0}$. It is confirmed in some studies that the presence of $\mathrm{TiO}_{2}$ and $\mathrm{ZrO}_{2}$ of the passive film in an alloy could increases its corrosion resistance $[489,494]$. Therefore, it is understandable that the $\mathrm{Ti}_{40} \mathrm{Zr}_{24} \mathrm{~V}_{12} \mathrm{Cu}_{5} \mathrm{Be}_{19} \mathrm{MGMCs}$ holds the higher passive film stability in the acid environment and has a great corrosion resistance.

Additionally, the corrosion properties of in-situ $\mathrm{Zr}_{58.5} \mathrm{Ti}_{14.3} \mathrm{Nb}_{5.2} \mathrm{Cu}_{6.1} \mathrm{Ni}_{4.9} \mathrm{Be}_{11.0} \mathrm{MGMCs}$ in the $\mathrm{NaCl}$ solution of various concentrations were studied by the potentiodynamic-polarization curves test [492]. The anodic and cathodic polarization curves in $\mathrm{NaCl}$ aqueous solutions with concentration varying from 1 wt. $\% \mathrm{NaCl}$ to $15 \mathrm{wt} . \% \mathrm{NaCl}$ in the ambient condition are shown in Figure 65(a) [492]. It can be seen that all of the potentiodynamic-polarization curves in solutions with different concentrations of $\mathrm{NaCl}$ have a similar profile. In the initial part of anodic polarization curves, for all the samples, the polarization curves exhibit a rapid increase in current densities, indicating an active dissolution state at the beginning of corrosion. 
With the further increase of the applied potential, the curves are starting to level off, suggesting the formation of passive films on the surface of Zr-based MGMCs. In other words, the dissolved passive elements were combined with the oxygen atoms in the vicinity to form a compact oxidation-protective film, which acted as a barrier for the anodic dissolution [493].

The impedance data of the Zr-based MGMCs specimens in different concentrations of $\mathrm{NaCl}$ solutions are shown in Figure 65(b) [492]. The electrochemical-impedance spectroscopy (EIS) curves of all samples are mainly composed of a capacitive reactance arc in the high-frequency region and an inductive impedance hook in the low-frequency range. Generally, the size of impedance arc is a good indicator for the corresponding corrosion resistance. By comparing the impedance-arc size, it is obvious that the sample exhibits the worst corrosion resistance in the $5 \% \mathrm{NaCl}$ solution, which is in good agreement with potentio-dynamic polarization measurements.

Besides, Gebert et al. [495] studied the pitting corrosion of in-situ dendrite / Zr-based MGMCs, and found that $\mathrm{Nb}$ and Ti components were beneficial in inhibiting the pitting initiation, due to their participation in the passive-film formation, and the dissolution of the glassy matrix phase was explained by the principal higher chloride reactivity of metastable phases, compared to that of stable crystalline phases and by the detected enrichment of $\mathrm{Cu}$ in these phase regions. Qin et al. [496] found that the high corrosion resistance of the $\left(\mathrm{Cu}_{0.6} \mathrm{Hf}_{0.25} \mathrm{Ti}_{0.15}\right)_{90} \mathrm{Nb}_{10}$ MGMC was attributed to the formation of $\mathrm{Hf}-$, Ti-, and $\mathrm{Nb}$-enriched highly-protective surface films during 
immersion in acid- and chloride-ion-containing solutions. The $\mathrm{Mg}_{69} \mathrm{Zn}_{27} \mathrm{Ca}_{4} \mathrm{BMGs}$ and ex-situ $\mathrm{Fe}_{\mathrm{p}} / \mathrm{Mg}_{69} \mathrm{Zn}_{27} \mathrm{Ca}_{4} \mathrm{MGMC}$ remarkably improved the corrosion resistance in the 3.5 wt.\% $\mathrm{NaCl}$ solution, compared with the AZ31 alloy and pure $\mathrm{Mg}$ [497].

Furthermore, with the view to the potential use as bioresorbable metals for orthopaedic applications, the corrosion behavior of the biodegradable lightweight BMGs and high-entropy BMGs is investigated [498-500]. Although the advantage of the corrosion behavior of MGMCs has been investigated, more deep understanding on corrosion mechanisms is badly needed. 


\section{Composite Thin-film Metallic Glasses}

With increasing interest in developing new family of BMG materials, thin film metallic glasses (TFMGs) is prepared due to their increased GFA. The investigation on TFMGs starts from 1980s, mainly focusing on immiscible binary systems, including $\mathrm{Cu} \backslash \mathrm{Ta}, \mathrm{Cu} \backslash \mathrm{W}, \mathrm{Cu} \backslash \mathrm{Zr}, \mathrm{Al} \backslash \mathrm{Fe}, \mathrm{Bi} \backslash \mathrm{Fe}$, and Bi $\backslash \mathrm{Ti}$ systems [501-503], together with the binary systems on annealing-induced solid-state amorphization (SSA) of multilayer thin films [504-506]. For example, in 1983, Schwarz and Johnson [504] fabricated the first La $\backslash \mathrm{Au}$ TFMG by SSA after annealing of the evaporated $\mathrm{La} / \mathrm{Au}$ multilayer films. Since late 1990s, TFMGs have received much attention in the scientific research for potential applications, due to the development of BMG alloys with good GFA in Pd-, Zr-, Cu-, Ti-, Mg-, Ln-, Fe-, and Ni-based alloy systems [507]. Moreover, the GFA of thin films can be further improved, since the vapor-to-solid deposition process of thin films are expected to deviate farther from equilibrium than the liquid-to-solid casting process $[508,509]$. Here, the annealing-induced SSA thin film is named as composite thin film metallic glass (CTFMG). Until now, TFMGs and CTFMGs with various thin-film thicknesses have been extensively employed to improve the performance of various substrate materials, especially in the field of mechanical behavior and corrosion resistance, which will be reviewed in this section $[438,510-516]$.

\subsection{Thin-film metallic glasses}

Numerous studies have been reported that the mechanical behavior of the film / substrate system is closely dependent on film properties, such as composition, 
thickness, hardness, ductility, structure, and film / substrate interfacial adhesion [438, $514,515,517,518]$. For instance, many ceramic coatings are susceptible to the fatigue-induced fracture and delamination under high stress levels, because of their intrinsically-brittle nature, despite their high hardness and strength. Moreover, with an increase in the coating thicknesses, a reduction in the adhesion of the film to the substrate may occur due to increased residual stresses [438, 519]. Five film properties have been reported to affect the mechanical performance, including fatigue and plasticity, of TFMG materials [438, 510, 513-515, 520, 521]: (i) hardness - to prevent surface roughening; (ii) ductility - to retard crack initiation at the sites where persistent slip bands (PSBs) intersect with the film; (iii) cyclic work hardenability - to reduce slip localization; (iv) residual compressive stresses - to decrease film tensile stresses; and (v) film / substrate adhesion - to prevent film delamination, which is one of the most important factors responsible for the mechanical properties enhancement of TFMGs. Therefore, a good balance between the beneficial properties of thin-film and film / substrate adhesion is the challenge to design the TFMG-substrate material systems.

Based on reported results, the TFMG can be a good candidate as excellent coating materials, due to their unique properties, as shown in Figure 66 [438]. It can be observed that TFMGs have much higher yield strengths than ceramic and metallic coatings, while metallic coatings and TFMGs have much larger ductility, usually indicating better film/substrate adhesion. Therefore, recently, TFMGs have been employed to improve the plasticity, strength, and fatigue life of different substrate materials, including steels [514, 515, 521, 522], Ni-based alloys [515, 520, 521], and 
BMGs [513], which is summarized in Figure 67 [438]. Figure 67(a) shows that the fatigue lifetimes and endurance limits of the aforementioned substrate materials coated with different thin films, together with monolithic $\mathrm{Zr}_{55} \mathrm{Cu}_{30} \mathrm{Ni}_{5} \mathrm{Al}_{10}$, and $\left(\mathrm{Cu}_{60} \mathrm{Zr}_{30} \mathrm{Ti}_{10}\right)_{99} \mathrm{Sn}_{1}$ BMGs for comparison. It can be observed that five regions (A, B, $\mathrm{C}, \mathrm{D}$, and E) are divided for the four-point-bending fatigue data, in which $\mathrm{A}, \mathrm{B}$, and $\mathrm{C}$ represents for low (smaller than $300 \mathrm{MPa}$ ), medium (300 - $700 \mathrm{MPa}$ ), and high (larger than $700 \mathrm{MPa}$ ) fatigue-endurance limit regions in the TFMG materials. Region D includes the tension-tension fatigue data of TFMG materials, while the four-point-bending fatigue data of monolithic BMGs is shown in region E.

For the high fatigue-endurance limit materials (region C), e.g., 316L stainless steel, the lifetime and fatigue-endurance limit can be slightly improved by Zrand $\mathrm{Cu}$-based TFMGs, which is caused by the high fatigue-endurance limit of substrates, despite of the good adhesion between film and substrate [523]. However, fatigue properties cannot be improved by the Fe-based TFMG, TiN, and pure-Cu films, which is generally attributed to the intrinsic brittle nature of films or poor film / substrate adhesion.

For the medium fatigue-endurance limit materials (regions B), e.g., HAYNES C- $2000^{\circledR} \mathrm{Ni}$-based alloys, the fatigue lifetime and endurance limit can be significantly enhanced due to the existence of Zr-based TFMGs, as exhibited in Figure 67(b) [438]. For instance, the fatigue-endurance limit is increased to $625 \mathrm{MPa}$ from 400 MPa. However, for the tension-tension fatigue data (region D), the fatigue behavior of 316L stainless steel cannot be significantly improved by $\mathrm{Zr}$ - and $\mathrm{Cu}$-based TFMGs, 
which is due to the poor film adhesion with the substrate, resulting from stress concentration in the notched dog-bone tension-tension specimens.

\subsection{Composite thin-film metallic glasses}

Amorphization in solids can be achieved by multi techniques, including mechanical alloying, high pressure, or shock loading [515]. For amorphization in sputtered TFMGs, SSA method has been employed by annealing the TFMGs in the temperature range of the supercooled liquid region $(\Delta \mathrm{T})$, which is between the glass transition temperature $\left(\mathrm{T}_{\mathrm{g}}\right)$ and the crystallization temperature $\left(\mathrm{T}_{\mathrm{x}}\right)$ [524-527]. For example, after annealing at $743 \mathrm{~K}$ (within $\Delta \mathrm{T}$ ) for various time periods of $\mathrm{Cu}$-based TFMG, the annealed specimens are basically either fully amorphous or nanocrystalline [527], but the annealing process for SSA needs to be carefully controlled, such as annealing temperature and time.

Due to the small scale of TFMGs and the existence of nanocrystalline phases, amorphization structure characterization on thin films is a very challenging task. Therefore, transmission electron microscopy (TEM) can be a very important tool for the microstructural and crystallographic analyses of TFMGs and CTFMGs, due to the micro-scale and nano-scale capability of TEM. In Figure 68 [525], the bright-field TEM images and electron diffraction patterns of $\mathrm{Fe}_{65} \mathrm{Ti}_{13} \mathrm{Co}_{8} \mathrm{Ni}_{7} \mathrm{~B}_{6} \mathrm{Nb}_{1}$ TFMGs can be observed, which suggests that minor nanocrystallites are formed after as-deposited film is annealed at the temperature $673 \mathrm{~K}\left(T_{\mathrm{g}}\right)$, and eventually transform into the fully amorphous phase at a temperature within $\Delta \mathrm{T}$ (i.e., $773-823 \mathrm{~K}$ ). However, annealing at a temperature above $T_{\mathrm{x}}$ (i.e., $873-1,023 \mathrm{~K}$ ) will lead to the appearance of 
crystallization again and the grain growth of FeNi phase in thin films. Besides the phase transformation, SSA can result in smoother film surfaces after annealing at a temperature within $\Delta \mathrm{T}$, whereas the surface becomes rougher as the annealing temperature increases due to crystallization and grain growth, which can be clearly characterized by atomic force microscopy (AFM) images in Ref. [525]. Moreover, similar SSA process is also reported on the $\mathrm{Cu}_{51} \mathrm{Zr}_{42} \mathrm{Al}_{4} \mathrm{Ti}_{3}$ TFMG after annealing at various temperatures [527]. Nanocrystalline $\left(\mathrm{Cu}_{10} \mathrm{Zr}_{7}\right)$ exist in the TFMG matrix after annealing at $673 \mathrm{~K}$, and become fully amorphous at $711 \mathrm{~K}$, whereas nanocrystalline phase appears again after heating at $798 \mathrm{~K}$.

In the reported literatures $[514,515]$, the mechanical behavior of TFMG / substrates can be improved, however, the investigation on mechanical performance of this SSA induced CTFMG is still quite limited, which will be reviewed in the current work. For example, hardness and elastic modulus of TFMG $\left(\mathrm{Zr}_{46} \mathrm{Ti}_{26} \mathrm{Ni}_{28}\right)$ can be measured using nanoindentation [528]. It is found that the hardness and elastic modulus of the as-deposited specimen are $6.2 \mathrm{GPa}$ and $107.0 \mathrm{GPa}$, respectively, and increased to 11.1 GPa and 140.1 GPa, respectively, for annealed (723 K) TFMG. Moreover, serrations in the load-displacement curves of TFMG specimens can be observed, but not as apparent as those in BMGs [247, 248, 251, 262, 275, 529]. Since the serration is related with the shear-band formation during loading deformation [251], the nanoindentation is thought to yield little and no shear bands generated upon loading, which is consistent with reported experimental works [530, 531]. 
Besides the nanoindentation characterization, fatigue behavior of the SSA

CTFMGs is also investigated, as shown in Figure 69 [515], which shows the S-N curves of 316L stainless-steel substrates with and without annealed TFMFs. The annealing temperature is $773 \mathrm{~K}$, since the $T_{\mathrm{g}}$ and $T_{\mathrm{x}}$ of this $\mathrm{Zr}_{53} \mathrm{Cu}_{29} \mathrm{Al}_{12} \mathrm{Ni}_{6}$ TFMG are $737 \mathrm{~K}$ and $796 \mathrm{~K}$, respectively, with a $\Delta \mathrm{T}$ of $59 \mathrm{~K}$. It can be observed that both as-deposited and annealed TFMG specimens exhibit better fatigue behavior, including lifetime and endurance limit, than the bare 316L steel substrate. The fatigue-endurance limit is increased to $\sim 700 \mathrm{MPa}$ and $\sim 750 \mathrm{MPa}$ for the as-deposited and annealed TFMG specimens, respectively, from $\sim 600 \mathrm{MPa}$ for the bare 316L steel substrates.

\subsection{Simulations on TFMGs}

As discussed in previous sections, the plastic flow in metallic glasses is accommodated by shear bands under loading, thus leading to catastrophic failure due to unconstrained propagation of individual shear bands. The most efficient way to improve ductility of BMGs is to geometrically constrain these shear bands, so that the plastic strain in each shear band can be reduced, therefore, slower the initiation of cracks. Coating is a typical approach to constrain the shear-band propagation and, thus, promote the proliferation of shear bands [532]. The detailed mechanism responsible for the coating effects can be well understood through computational studies, such as finite-element modeling [178, 533-535] and molecular-dynamic (MD) simulation [515, $536]$.

\subsubsection{Molecular-dynamic simulations}


Molecular-dynamic simulations have been applied widely in studying the deformation mechanisms at atomic scales in materials, including thin film metallic glasses [537]. For example, the medium-range order of MGs investigated with MD simulations has been discussed in Ref. [148]. Moreover, Wang et al. [536] build the MD model for film sputter deposition and 3-D indentation simulations, in which the interface between the substrate and film can be modeled realistically. During sputter deposition process, MD simulations can be employed to construct the initial film configuration. Figure 70(a) [515] shows the results of an as-deposited $\mathrm{Cu}_{47} \mathrm{Zr}_{47} \mathrm{Al}_{6}$ TFMG, in which atoms are represented as colored circles ( $\mathrm{Al}$ is red, $\mathrm{Cu}$ is green, and $\mathrm{Zr}$ is blue). The substrate is Ti and the film thickness is $80 \AA$. During the deposition simulation, deposited atoms are allowed to penetrate into the substrate while forming the TFMG / substrate interface.

In the MD-simulations of deposition and indentation process, interatomic potentials were adopted to probe the interactions among the three species of atoms forming the metallic glass. The interatomic potentials are expressed as:

$$
E_{i}=-\left\{\sum_{j} \xi^{2} \exp \left[-2 \mathrm{q}\left(\frac{r_{i j}}{r_{0}}-1\right)\right]\right\}^{\frac{1}{2}}+\sum_{j} A \exp \left[-\left(\frac{r_{i j}}{r_{0}}-1\right)\right]
$$

where $\xi$ is an effective hopping integer, $\mathrm{r}_{\mathrm{ij}}$ is the distance between atoms $\mathrm{i}$ and $\mathrm{j}, \mathrm{r}_{0}$ is the first-neighbor distance, and $\mathrm{A}(\mathrm{eV}), \mathrm{p}, \mathrm{q}$, and $\xi(\mathrm{eV})$ are system-specific parameters, which is detailed explained in Ref. [538]. During film deposition, the interaction among the protection gas, $\mathrm{Ar}+$, and neutral atoms is governed by the pair-wise Moliére potential, $\mathrm{V}_{\mathrm{ij}}(\mathrm{eV})$ : 


$$
V_{i j}(\mathrm{r})=\frac{Z_{1} Z_{2} e^{2}}{r_{i j}}\left(0.35 \exp \left[\frac{-0.3 r_{i j}}{L_{f}}\right]+0.55 \exp \left[\frac{-1.2 r_{i j}}{L_{f}}\right]+0.1 \exp \left[\frac{-6 r_{i j}}{L_{f}}\right]\right)
$$

where $L_{f}$ is the Firsov screening length, and e is the electron charge. $Z_{1}$ and $Z_{2}$ are the atomic numbers of the gas ion and neutral atom, respectively.

The MD-simulation result of indentation on as-deposited $\mathrm{Cu}_{47} \mathrm{Zr}_{47} \mathrm{Al}_{6}$ TFMG is exhibited in Figure 70(b) [515]. In this graph, all atoms are color coded according to their engineering strain $\varepsilon_{11}$, in which red and blue colors represent for tension and compression strain, respectively. Here, the atomic strains are calculated from the atom positions as a function of time via a discrete deformation gradient tensor. It can be observed that atom pileup appears around the indenter, indicating homogeneous flow of the metallic glass under intensive stress. Radial shear bands (red or blue lines extending from the indenter) can be noticed where plastic flow occurs.

From the aforementioned MD simulation results, the connection between STZs and atomic shear bands can be understood. At the initiation stage of indentation, STZs can be activated at "weak spots" around the indenter. Here, the "weak spots" is defined as the loosely packed atomic regions, where embryonic shear bands will be initiated. With load increasing, embryonic shear bands will evolve into mature shear bands, while the original "weak spots" may become parts of the plastic-flow regions.

\subsubsection{Finite-element modeling}

As discussed above, MD simulations can be an effective method to study the atomic structure in metallic glass thin films. However, for the mechanical behavior and shear banding of metallic glasses, finite-element modeling (FEM) could be a better choice $[438,534,535]$. As reported in literature [514, 515], the mechanical behavior of 
the film / substrate material system is significantly dependent on the film / substrate adhesion, as well as the film thickness, which can be simulated with a free-volume model.

To explore the effects of geometry constraints, including coating thickness and adhesion, on plasticity enhancement of substrates, a two-dimensional (2-D) indentation model can be constructed with ABAQUS to investigate the film / substrate (MG substrate and Ti coating) material systems with varied configurations and constitutive laws. More information about this model can be found in Ref. [438]. The MG substrate can be simulated with the free-volume model, which is described in detail in Ref. [535, 539], while the Ti coating is treated as a pure elastic body.

To investigate the effects of film / substrate adhesion on the ductility enhancement of metallic glass, different indentation contours were displayed in Figure 71 [438]. In Figure 71(a), several major intersecting shear bands appear upon the indentation of the bare BMG substrate, while, for Ti-coated BMGs, multiple shear bands can be observed after indentation for both good-adhesion and poor-adhesion cases, as exhibited in Figures 71(b) and (c), respectively. This shear-band multiplication phenomenon in film / substrate materials is attributed to two mechanisms triggered by the geometrical constraint of thin films: (i) shear-band reflection occurs at the film/substrate interface, and (ii) abundant minor shear bands exist in the BMG substrates. According to the experimental results [513], the shear-band multiplication is general corresponding to the improved plasticity of the BMG substrate. Regarding the poor film / substrate adhesion case [Figure 71(b)], less 
shear-band reflection and branching can be observed compared to the good film / substrate adhesion case [Figure 71(c)], which is generally attributed to the film delamination occurring easily in the poor adhesion case, and, therefore, relatively limited enhanced plasticity can be obtained in the poorly-bonded thin film material.

How is the shear-band propagation direction in the coating / substrate (BMG) materials under deformation? It can be calculated with Rudnicki-Rice instability theory, which is constructed with a 3-D half-symmetric ABAQUS model under Rockwell indentation, as displayed in Figure 72(a) [438]. The predicted directions of shear-band propagation for both monolithic and thin-film coated BMGs are displayed in Figures 72(b) and (c) [438], respectively. The detailed information for the model setup can be found in Ref. [534]. From the Rudnicki-Rice instability theory, the calculation gives typical radial shear-band patterns under indentation for both bare substrate and coating specimens, which is consistent with the experimental data [534, 540, 541]. For the bare BMG substrate [Figure 72(b)], only major shear bands appear and propagate through the specimen, due to lack of shear-band constraint conditions. However, for the coated specimen [Figure 72(c)], more shear bands occur in the substrate upon deformation, resulting in reduced shear strain in each shear band. Note that in Figures 72(b) and (c), the blue solid curves represent the predicted shear-band directions, while the red dashed curves are along principal shear stress directions. Moreover, in the coated BMG [Figure 72(c)], shear bands can be "reflected", resulting in the appearance of more minor shear bands [solid curves], when major shear bands propagate to the film / substrate interface. Note that the short solid black and dashed 
green curves are corresponding to the shear-band directions and principal shear-stress directions, respectively, occur at the coating / substrate interface.

Besides, the electrodeposition was used to coat $\mathrm{Cu}$ films on the surface of the $\mathrm{Zr}_{52.5} \mathrm{Cu}_{17.9} \mathrm{Ni}_{14.6} \mathrm{Al}_{10} \mathrm{Ti}_{5}$ (Vit 105) BMG pillars with the film thicknesses of 71.5 and $161.1 \mu \mathrm{m}[542]$. The plastic strain was $6.1 \%$ for the coated pillars with a coating thickness of $161.1 \mu \mathrm{m}$, which is 3.59 times of $1.7 \%$ of the bare Vit 105 pillars. The deformation of the copper films dissipated the strain energy and limited the propagation of shear bands, which led to the initiation and formation of multiple shear bands. Similar function works for Ni film / Vit 105 BMG composites [543]. Therefore, the enhanced plasticity can be obtained in thin-film coated BMG materials. 


\section{Deformation mechanisms and models of metallic glass matrix composites}

The deformation behaviors of ex- and in-situ metallic glass matrix composites at room temperature are discussed in detail above, and the corresponding deformation mechanisms are also explored in part, especially for that of ex-situ composites. In this section, we will mainly have a discussion on the deformation mechanism of in-situ composites at room temperature upon quasi-static uniaxial loading.

\subsection{Deformation mechanisms under compression}

For in-situ dendrite-reinforced MGMCs, the stress-strain curve of $\mathrm{Zr}_{58.5} \mathrm{Ti}_{14.3} \mathrm{Nb}_{5.2} \mathrm{Cu}_{6.1} \mathrm{Ni}_{4.9} \mathrm{Be}_{11.0}$ composites under compression is shown in Figure 35, and the side view of the deformed sample reveals the distribution of profuse shear bands [544]. Simply, it is usual to correlate the plasticity and the density of shear bands. More comprehensive micromechanisms are badly needed to explore.

Figure 73(a) [544] shows the TEM bright-field image and the corresponding selected-area-electronic-diffraction pattern (SAED) for the dendrites in the deformed composites (inset). Using TEM, the shear bands, observed from the SEM image of the deformed sample, are neither large in number nor in width, making them difficult to be caught in the TEM-sample preparation. Theoretically, the width of shear bands is estimated to be about $10 \mathrm{~nm}$ [36]. Meanwhile, experimentally, the value accords to the theoretical value [545]. The slip bands in the dendrites, seeming to be of a high number density, denoted by the white arrows, can be frequently observed. Most of 
these bands propagate inside the dendrite with a spacing of $70-140 \mathrm{~nm}$, and the propagating direction has no special relationship with the compression direction. The spacing is in reasonable agreement with that observed in a micrometer-sized dendritic phase intermixed with a nanostructured eutectic matrix [546]. Two slip systems are activated with mutually perpendicular directions. In a given dendrite, the slip bands generally extend along the same direction. The extensive slip deformation induces the stepped morphology, denoted by the black arrows in Figure 73(a), at the interface between the dendrites and the glass matrix. The SAED pattern in the inset of Figure 73(a) is identified as the $\left[\begin{array}{lll}1 & 1 & 3\end{array}\right]$ zone axis of the bcc $\beta-\mathrm{Zr}$ with a lattice parameter, $a=$ 3.240 Å. Figure 73(b) [544] shows the details about the deformation of dendrites. The inset in Figure 73(b) is the TEM bright-field image of a dendritic arm with high magnification. It can be observed that along the slip direction, steps denoted by the black arrows at the interface form due to the plastic deformation in order to readily accommodate the plastic strain. Thus, these steps can be considered as "misfit" between the two phases to harmonize strains. Figure 73(b) presents a low-magnification HRTEM image of dendrites taken far away from the interface, in which some Moiré patterns denoted by black arrows emerge, and the area of the Moiré pattern is approximately $20 \mathrm{~nm}$. One can observe the typical contrast in the dendrites, which is caused by the strain of shear bands, indicating the shear direction and identifying the penetration of shear bands to the dendrites. The direction of the Moiré patterns does not follow a certain direction, since the Moiré patterns are generated not 
only by translation but also by a mixture of translation and rotation due to the local superposition subjected to the complicated shear deformation.

Figure 73(c) [544] displays the HRTEM image near the interface. Abundant lattice distortion fringes prevail in the dendrite marked by the arrows, indicating the lattice of the $\beta-\mathrm{Zr}$ phase is severely distorted. The fast Fourier transformed (FFT) pattern from the area of the dendrite marked by the rectangle is shown in the inset of Figure 73(c), and the corresponding inverse fast Fourier transformed (IFFT) pattern is displayed in Figure 73(d) [544]. From Figure 73(d), it is clearly demonstrated that the severe lattice distortion denoted by arrows is yielded to alleviate the stress concentration. The deformation-induced lattice distortion is widely observed in crystalline alloys [547], which has not been investigated in MGMCs previously. Active dislocations are around the area located in the severe lattice distortion with a high number density, giving an evidence of pile-ups of dislocations at the interface. Besides, the amorphous-like region marked by the circle in Figure 73(d) is found, indicative of the local disordered amorphization in dendrites. Figure 73(e) [544] illustrates a HRTEM image of the deformed glass matrix. A nano-sized crystal with the size of about $3 \mathrm{~nm}$ is found, and its corresponding IFFT pattern [inset in Figure 73(e)] gives a periodic lattice contrast. The nano-crystallites in the matrix are seldom found, which cannot be responsible for the improved plasticity and the work-hardening capacity. In summary, the improved plastic deformation of in-situ dendrite composites is mainly characterized by the multiplication of shear bands. Severe lattice distortion and local amorphization in the dendrites, as well as pile-ups 
of dislocations close to the interface, are jointly responsible for the micromechanisms of the compressive deformation.

Besides, Wang et al. [548] investigated the role of the interface before and after deformation in an in-situ Ti-based MGMC by using TEM and HRTEM, and found the interface has the positive effect to initiate nucleation of multiple shear bands in glass matrix through nanocrystallization or to cause plastic deformation by dislocations and the disordered atomic arrangement parts in dendrites. In-situ synchrotron x-ray experiments have been used to follow the evolution of the diffraction peaks for crystalline dendrites embedded in a metallic glass matrix subjected to a compressive loading-unloading cycle [549]. We observe irreversible diffraction-peak splitting even though the load does not go beyond half of the bulk yielding strength. The chemical analysis coupled with the TEM mapping suggests that the observed peak splitting originates from the chemical heterogeneity between the core (major peak) and the stiffer shell (minor peak) of the dendrites. A molecular dynamics model has been developed to compare the hkl-dependent microyielding of MGMCs. The complementary diffraction measurements and the simulation results suggest that the interface, as Maxwell damper, between the amorphous matrix and the (211) crystalline planes relax under prolonged load that causes a delay in the reload curve, which ultimately catches up with the original path. The core-shell structure is firstly found in in-situ dendrite composites, which plays an important role in determining the mechanical response upon loading. The more detailed functions should be explored furthermore. 
In addition, the micromechanics of deformation of in-situ formed MGMCs consisting of Ta-rich particles dispersed in an amorphous matrix was examined [550]. The development of a plastic misfit strain causes stress concentrations around the $\mathrm{Ta}$ particles, resulting in localized yielding of the matrix by shear banding at an applied stress of 1,450 MPa, considerably lower than the macroscopic yielding strength of the composite $(\sim 1,725 \mathrm{MPa})[550]$. The presence of the particles makes the stress state highly inhomogeneous, which may partially explain why fracture is suppressed in the composite, allowing the development of large plastic strains. Similar stress concentration happens in ex-situ MGMCs, splitting or shearing for ex-situ MGMCs upon compressive loading has been discussed above [296].

\subsection{Deformation mechanisms under tension}

It should be noted that most of the developed in-situ MGMCs exhibit softening upon tension rather than work hardening upon compression [94, 97, 101, $105,164,549,551]$, giving an implication that the tensile mechanism may be very different from the corresponding compressive one. The challenge for structural applications is how to obtain the tensile ductility and work-hardening capacity. Only if the materials can be homogeneously plastically deformed, the localized deformation and softening leading to the early failure can be avoided. It is necessary to know the tensile deformation micromechanisms of MGMCs. The discussion below is based on the tensile micromechamisms [101].

The overview of the TEM BF image of the deformed sample is exhibited in Figure 74(a) [101]. It is seen that the dendrite fragmentation prevails after tension, 
and the sharp shearing within the dendrites, denoted by the arrows, takes place without predominant orientation. Since each individual dendrite has its own crystallographic orientation, the orientation-dependent critical shear stress is activated at different spatial and temporal scales. Consequently, the plastic deformation of individual dendrites is harmonized, leading to a random fragmentation. Figure 74(b) displays the HRTEM image of the glass matrix after deformation, and only a few maze-like patterns less than $5 \mathrm{~nm}$ are available, in agreement with the SAED result in the inset of Figure 74(b). It is reasonable to deduce that the rarely-found nanocrystallization can't be responsible for the distinguished tensile ductility [101]. Figures 74(c) and (d) exhibit the bright- and dark-field (BF and DF) images of the dendrites, respectively [101]. Compared to the two images, three subdivisions indicated by , , and are separated by the shearing boundaries, marked by arrows. The formation of shearing boundaries suggests that the proceeding shear bands cut into the dendrites. Different contrasts between these subdivisions originate from the various orientations, since they may experience the varied plastic deformation. For the further identification of the varied deformation, the lattice parameter, $a$, is investigated for neighboring subdivisions and . The SAED patterns taken from the rectangular regions in subdivisions and are shown in Figures 74(c) and (d), respectively. $a=0.3175$ and $0.3221 \mathrm{~nm}$ are obtained for subdivisions and , respectively, suggesting the experience of different plastic deformation. The most heavy deformation concentrates within the thin shear bands with a thickness of about $30 \mathrm{~nm}$. Figure 74(e) displays the IFFT patterns within the shear bands, and it is found 
that dislocations, denoted by “ $\perp ”$, lattice distortions, represented by arrows, and local amorphization, represented by ellipses, are together present.

In addition to the shearing-induced separation within individual dendrites, there are many subdivisions of the dendrites, separated by the dense dislocation walls (DDWs). Figures 74(f) and (g) show the BF and DF images of the dendrites separated by DDWs, indicated by arrows, respectively. The cell blocks (CBs) form with the DDWs as the boundaries due to the harmonized deformation, since the amount of slip systems within CBs is different. Compared to the BF and DF images, the fragmentation dominates, and the varied contrasts from the different $\mathrm{CBs}$ indicate various orientations. It is the first time to observe the fragmentation of dendrites in BMGMCs, divided by the DDWs, but similar fragmentation has been reported in UFG $\mathrm{Cu}$ alloys [552]. Additionally, the shear steps can be observed, as shown in Figure 74(f), giving an evidence of the interaction between the shear bands and the deformation structures. CBs A and B can be easily determined, based on the contrast in bright- and dark-field images. Actually, even within the CB B, the gradual contrast is still present. The high-magnified BF image of the $\mathrm{CB} B$ is exhibited in the inset of Figure $74(\mathrm{~g})$, and microbands are generated due to the local stress concentration during the plastic deformation. The magnified DDW between the CBs $A$ and B is shown in Figure 74(h). The disorder lattice pattern within the CB A appears to be characteristic of an amorphous state, which is caused by the notable misorientation between CBs A and B. The FFT pattern taken from the DDW, displayed in the inset of Figure 74(h), produces extra diffraction spots, marked by 
arrows, according to the large misorientation. Besides, Moiré patterns emerge within the $\mathrm{CB} B$.

The study above emphasizes the interior of the dendrites, and it is concluded that the interior fragmentation results from subdivisions separated by the shear bands and DDWs. Next, the direct observation of the fragmentation or fracture of the dendrites is performed. Figure 74(i) exhibits a superelongation of local dendrites under Mode . The continuous necking dominates during plastic deformation, and both necking tips shrink into nanoscales with a displacement of about $33 \mathrm{~nm}$. The local tensile ductility is an indicator of the macroscopically-tensile ductility. Additionally, the local shearing under Mode results in the separation of adjacent portions of the dendrites, as shown in Figure 74(j). Sharp breaks in the dendrites form, and the two fracture planes are not well parallel, together with a distinct distance. Figure $74(\mathrm{k})$ is a HRTEM image taken near the interface of the dendrite and the glass matrix, and no atomic-scale segment is found, which suggests a good structural coherency of the interface, and indicates that the harmonized plastic deformation in the dendrites may extend into the local glass matrix. For the dendrite near the interface, the deformation structure is clear, as shown in an IFFT pattern in Figure 74(1), which is analogous to Figure 74(e), where dislocations, lattice distortions, and local amorphizations are present. Based on the fracture study of the BMGMCs, shearing-induced subdivisions and DDW-separated CBs constitute the deformation structure within the interior of dendrites. Besides, the local separation under Modes and prevails. 
Additionally, the investigation of the microcrack evolution in an in-situ Ti-based MGMC was conducted by Wang et al. [553] Figure 75 [553] demonstrates the deflection of the extending direction. Apparently, the extension direction of a microcrack from the amorphous phase to the dendrite diverges to the dendrites, along "mn" in Figure 75(a), and leads to a nanoviods along the initial direction. However, the extending direction changed again, slide along the interface instead of the propagation along "mn". Figure 75(b) shows that the microcrack propagates along the plastic zone (length scale $\sim 3 \mu \mathrm{m}$ ), including the plastic deformation zone and shear deformation zone, "mn", and the extension direction slightly deviates from the original crack direction. Generally, it is difficult to observe the propagation of shear bands upon loading, since the formation and propagation of the primary shear bands in monolithic BMGs occur simultaneously under tensile loading. The current study gives a direct evidence of the evolution of shear bands to cracks.

\subsection{Deformation model by simulations}

Unlike the well-defined long-range order that characterizes crystalline metals, the atomic arrangements in amorphous alloys remain mysterious at present. As a result, up to now, the elucidation of the different types of short-range order as well as the nature of the medium-range order has been mainly limited in binary and ternary alloy systems $[148,554]$. Correlation of atomic structures with the mechanical behavior was well established in these alloy systems [257, 555-557]. However, the deformation models are difficult to be established in MGMCs, since multicomponents and more than one phase are present in MGMCs. 
In order to reveal the mechanisms of the improved ductility in MGMCs, the maximum shear stress fields defined as: $\tau=\sqrt{\left(\sigma_{x x}-\sigma_{y y}\right)^{2}+\sigma_{x y}^{2}}$, are analyzed as shown in Figure 76 [558], where $\sigma_{x y}$ are stress components around the dendrite with fracture energy $e_{\mathrm{c}}=145.2 \mathrm{~J} / \mathrm{m}^{2}$. Figures 76(a) and (b) show $\tau$ around the dendrite with the rotation angles $\theta=0 \mathrm{deg}$. and $\theta=15 \mathrm{deg}$., respectively. It can be seen in Figure 76(b) that the un-symmetric shear stress at the tip of incident shear bands causes the detour of shear band upon touching the secondary arm of dendrites with a rotation angle $\theta=15 \mathrm{deg}$. On the contrary, the shear stresses are symmetric around the tip of shear band touching the primary arm of dendrite with a rotation angle $\theta=15 \mathrm{deg}$., resulting in the branching of the incident shear bands, as shown in Figure 76(a).

The successful application of the phase-field approach on the understanding of shear banding and fracture processes of ex-situ fiber-reinforced MGMCs is achieved by Zheng et al. [408, 559]. Multiple shear bands can be initiated from the interface. The enhanced fracture toughness of the composite is related to the bonding conditions between the glass matrix and the fibers and its relation with the free-volume defect at the interface is quantitatively determined. From the simulation study, it is found that the integration of two attracting shear bands will result in the generation of secondary shear bands, leading to enhanced plasticity [559].

For the in-situ ductile-phase-reinforced MGMCs, by simulations, Abdeljawad et al. [560] found that the ductility is inversely proportional to the ductile particle size. For $\mathrm{Cu}_{64} \mathrm{Zr}_{36}$-matrix composites, Zhou et al. [327] revealed atomistic details related to the evolution of plastic shearing events in the glass matrix, as well as 
the interactions between the glass matrix and the crystalline phase in simulations. The interaction is governed by a cooperative activation of lattice sliding and local STZs in the vicinity of the glass-crystal interface. By matching this structural length scale to the intrinsic length scales of the glass matrix, one can obtain global plasticity (homogeneous nucleation of STZs or initiation of multiple shear bands) in the composites. It is also found that slender crystalline secondary phases are better at suppressing shear-band propagation than those with spherical shapes. Besides, based on the interaction between the dislocation-sliding and shear-banding, numerical simulations were performed to elucidate the toughening mechanisms of particle-reinforced MGMCs under tension [561]. During numerical modeling, the free-volume theory was applied for the glass matrix, and free volume density acts as an internal state variable to describe the evolution of shear banding. While the mechanism-based strain gradient plasticity theory was adopted to describe the crystalline second phase, and dislocation density was employed to track the dislocation sliding. Jeon et al. [562] used the finite-element-method (FEM) analysis based on real microstructures to theoretically explain the enhanced elongation in terms of effective dendrite size in in-situ dendrite / MGMCs. The shape and location of deformation bands estimated from the FEM simulations were well matched with the experimental observations. The more precise resolution to the micromechanism by simulation needs to be with the aid of electron backscatter diffraction (EBSD) methods, since it has been confirmed that the plastic deformation produced indexable patterns and there was a preferred texture, and dendrites oriented with a $<101>$ parallel to the tensile axis were 
least likely to experience plastic deformation that would result in Kikuchi pattern degradation [563]. Hardin et al. [328] have investigated various microstructural factors (volume fraction, length scale, and yielding strength) influencing strain delocalization in in-situ MGMCs. The modeling coupled with experiments could be a useful tool for the design of strong and ductile MGMCs. 


\section{Applications}

From above descriptions, it is clear that BMGs or MGMCs have an interesting combination of properties. They exhibit very high strength (both in tension and compression), large elastic elongation limit, very high hardness, and excellent corrosion resistance, etc.

For the applications, Kumar et al. [564] discussed the advantages of using metallic glasses in miniature applications. Size-dependent properties suggest that applications on length scales from $1 \mathrm{~nm}$ to $5 \mathrm{~mm}$ would benefit the most from BMGs. These applications are in the fields of MEMS (microelectromechanical system), NEMS (nanoelectromechanical systems), biomedical implants, precision microparts, surgical tools, and micromachines.

As different applications require various forms of glassy materials, the BMG alloy compositions are produced in the form of rods, sheets, plates, spheres, pipes, etc. Figure 77 show some BMG-product photos fabricated directly from their molten state by DongGuan Eontec Co., Ltd. in China [565]. Figure 77(a) exhibits some jewelries made from BMGs. Figures 77(b), (c), and (d) show the photographs of mobile phone skeletons in different forms and shapes, in which $\mathrm{Zr}$ - and $\mathrm{Cu}$-based BMGs are synthesized (Note that all the products are not artificially polished). The high yielding (or fracture) strength, low Young's modulus, large elastic strain limit, and easy formability in the supercooled liquid region are the main attributes of BMGs that make them attractive for structural applications. This attribute of BMG alloys has been extensively exploited to produce different types of parts with complex shapes such as 
gears, coiled springs, and other complex parts [566]. The superior corrosion resistance of BMGs over their crystalline counterparts plays a major role in chemical applications. BMGs have been specifically considered as most appropriate materials for fuel cell separators. Fuel-cell systems are known to have a higher efficiency in comparison to internal combustion engines by directly converting the chemical energy of fuels to electrical energy. There has been recent progress in the development of proton-exchange membrane fuel cells (PEMFCs) by utilizing the superior corrosion resistance and viscous deformability of BMGs. Besides, in the field of biomedical applications, implants that 'biocorrode' are of considerable interest. Deploying them not only abrogates the need for the implantremoval surgery, but also circumvents the long-term negative effects of permanent implants [498-500, 567, 568]. In addition, the outstanding soft magnetic properties of the Fe-based melt-spun ribbons or BMGs have found applications in power-distribution transformers and several other applications [566]. Recently, Krautz et al. [569] reported on novel magnetocaloric composites based on $\mathrm{La}(\mathrm{Fe}, \mathrm{Si})_{13}$ particles in an amorphous metallic matrix, showing a promising route for the production of compact refrigerant bodies that can be easily extended to other brittle giant magnetocaloric materials whose performance depends strongly on particle size.

The broad application of BMGs will go on. In comparison, the high strength together with high toughness in MGMCs render them potential candidates for applications in the field of structural engineering. The following is a design route for the development of high-performance knives [570]. 
Quality knives are typically fabricated from high-strength steel alloys. Depending on the applications, there are different requirements for mechanical and physical properties that cause problems for steel alloys. For example, diver's knives are generally used in salt water, which causes rust in steel knives. Titanium diver's knives are a popular alternative due to their salt water corrosion resistance, but are too soft to maintain a sharp cutting edge. Steel knives are also magnetic, which is undesirable for military applications where the knives are used as a tactical tool for diffusing magnetic mines. The solution to the deficiencies of titanium and steel knives is to fabricate them using MGMCs. They can be cast into net or near-net shaped knives with a combination of properties that exceed both titanium and steels, which are based on titanium. The knife has a self-sharpening edge, and can retain an edge during service. And it is hard, non-magnetic, corrosion-resistant against a variety of corrosive environments, and tough (to allow for prying), which can be cast into a net-shape with a mirror finish and a complex shape with an excellent wear resistance, and low density, as shown in Figure 78 [570]. Examples of a variety of net-shaped parts of the Ti-based MGMC DV1 (TiZrVCuBe) show how complex shapes can be cast with Ti-based composites. Bolts, washers, hollow tubes, and rods are demonstrated in Figure 79 [571]. As presented in Section 1, various processing methods can be used to directly cast in-situ MGMCs with complex shapes, as shown in Figure 80 [572]. The structural applications in MGMCs bear similarities to the monolithic BMGs.

It should be noted that the special applications in strategic fields, such as the defense and aerospace, are for ex-situ $\mathrm{W}_{\mathrm{f}}$-reinforced MGMCs. The adiabatic 
shear-band failure mechanism of ex-situ $\mathrm{W}_{\mathrm{f}}$-reinforced MGMCs at high strain rates suggests that they can be used in the kinetic-energy penetrator applications. 


\section{Conclusions}

The development of various ex-situ and in-situ MGMCs is reviewed in this paper. Since the first amorphous alloy was synthesized in the Au-Si system in 1960, people are trying to search for the BMGs with the large glass-forming ability in different alloy systems. Up to now, there have been many alloy systems, such as $\mathrm{Zr}$, $\mathrm{Ti}$, $\mathrm{La}, \mathrm{Mg}, \mathrm{Cu}, \mathrm{Ni}, \mathrm{Fe}, \mathrm{Pd}$, and Gd-based etc, which have the critical diameters over than 1 $\mathrm{cm}$. These BMGs could be fabricated by various processing methods, including the copper-mould suction casting, Bridgman solidification, spark plasma sintering, electromagnetic-vibration process, pulsed-laser forming, low-pressure die-casting, melt atomization and spray deposition, continuous casting, etc. And the copper-mold suction casting is the most common method to fabricate BMGs, and most developed BMGs are based on this method all over the world.

Although a series of excellent mechanical properties have been explored gradually, the intrinsic room-temperature brittleness of monolithic BMGs is yet to be solved until the appearance of ex-situ and in-situ MGMCs since 1990s. After foundation of plastic-deformation models, such as free-volume, STZ, and TTZ models, the shear-banding behavior, which is associated with the catastrophic failure at room temperature, is widely accept to explain the brittleness. However, in ex-situ and in-situ MGMCs, the secondary phases can effectively hamper the fast propagation of shear bands, and the improved toughness at room temperature is generally obtained. It should be noted that there exists mechanical asymmetry during the quasi-static uniaxial compression and tension for not only BMGs but also MGMCs. Specially, there is 
almost no work hardening during tension of ex-situ and in-situ MGMCs, even if the large compressive plasticity together with the macroscopic work hardening is obtained. Based on this asymmetry, the deformation micromechanisms upon compression and tension are analyzed in detail. Besides, on the basis of the background that it is inevitable for the engineering materials serving upon dynamic loading, the dynamic compression, tension, and shear punching behaviors of ex-situ and in-situ MGMCs are studied.

The deformation behaviors of MGMCs at cryogenic and high temperatures are experimentally and theoretically discussed. The fatigue results of BMGs and MGMCs are summarized and compared. In particular, the four-point-bending and tension-tension results of in-situ dendrite / MGMCs and the corresponding mechanisms are carefully explored. Note that the fatigue behavior in shape-memory MGMCs is firstly reported here. Though the advancement has been achieved in the study of mechanical properties, deeper understanding, such as deformation models, should be developed in order to widen the potential applications. Apart from the deformation, the corrosion and wear properties of ex-situ and in-situ MGMCs have been mentioned. And the composite thin-film metallic glasses together with $\mathrm{Ni}$ - and $\mathrm{Cu}$-coated $\mathrm{BMGs}$ are investigated. The corresponding deformation model is illustrated by experiments and simulations. Lastly, the applications of MGMCs now and in future are discussed. 


\section{Future work}

Although numerous efforts have been paid on the in-situ and ex-situ

MGMCs during the past years, especially on the aspect of structural engineering, such as deformation, more work should be unfolded in order to pave a way for actual applications. Attempts to bridge high toughness and high strengths on the MGMCs have always been the goal of the previous, present, and future research.

The asymmetry in mechanical properties under compressive and tensile loadings exists, e.g., distinguished plasticity accompanied by the work-hardening behavior under compressive loading, but fully softening after yielding under tensile loading. For structural applications at room temperature, homogeneous deformation with the work-hardening behavior is highly expected. How to obtain homogeneous deformation is poorly understood to design MGMCs with high toughness. Based on these safety evaluations, deformation mechanisms should be explored extensively in the future. Besides, the service surrounding is complex for materials. More investigations on the deformation behavior can be concentrated under the high-speeding dynamic loading, such as dynamic compressive, tensile, and shearing loading at varied temperatures, such as cryogenic or high temperatures beyond the glass-transition temperature. Up to now, almost all the research activities on MGMCs are based on the as-cast samples. It is well known that it is inevitable to obtain micro-scaled casting pores in conventional alloys, and the rolling process is widely employed to remove the pores [573]. Previously, Cao et al. have demonstrated that the plasticity of BMGs after cold rolling is remarkably enhanced [574]. Thus, people may 
pay more attention to the rolling effect on MGMCs, which can remove the casting pores, and enhance the plasticity [575]. Additionally, lightweight is of interest for MGMCs with high specific strengths, such as Mg-, Ca-, and Ti-based MGMCs [571, $576,577]$

Except for the consideration on structural properties, functional properties should be vital [578], and developed for special applications, such as soft magnetism, high electroconductibility, high thermal conductivity, shape memory, etc.

\section{Acknowledgment}

All the authors thank Prof. Y.W. Chung's great invitation. We are indebted to many researchers in this field with whom we have had valuable and stimulating discussions, and whose work provided the inspiration for writing this overview. We wish to thank in particular Dr. M.Q. Jiang from the Institute of Mechanics, Chinese Academy of Sciences for providing the original artwork for the deformation model including TTZ and STZ theories, Dr. J. Bai from Northwestern Polytechnical University for his writing on fracture toughness, and Dr. H. Wu from the Central South University for his writing on wear behavior. Drs. Y.S. Wang and H.J. Yang from Taiyuan University of Technology give their contributions on the writings.

J.W.Q. would like to acknowledge the financial support of National Natural Science Foundation of China (Nos.51101110 and 51371122), the Program for the Innovative Talents of Higher Learning Institutions of Shanxi (2013), the Youth Natural Science Foundation of Shanxi Province, China (No. 2015021005), and the opening project of State Key Laboratory of Explosion Science and Technology (Beijing 
Institute of Technology), and the opening project number is KFJJ15-19M. P.K.L. would appreciate the National Science Foundation (DMR-0909037, CMMI-0900291 and CMMI-1100080), the Department of Energy (DOE) Office of Nuclear Energy's Nuclear Energy University Programs (NEUP, grant \#00119262), the DOE Office of Fossil Energy, NETL (DE-FE0008855, DE-FE-0011194, and DE-FE-0024054), with Drs C.V. Cooper, A. Ardell, Z.M. Taleff, R.O. Jenseng Jr, L. Tian, V. Cedro, R. Dunst, S. Lesica, J. Mullen, and S. Markovich as program managers, and the support from the U.S. Army Office Project (W911NF-13-1-0438) with the program manager, Dr S.N. Mathaudhu. 


\section{Reference}

[1] W. Klement, R.H. Willens, P. Duwez, Nature 187 (1960) 869-870.

[2] H.S. Chen, S.D. Ferris, E.M. Gyorgy, H.J. Leamy, R.C. Sherwood, Appl. Phys. Lett. 26 (1975) 405-406.

[3] H.S. Chen, Acta Metall. (1974) 1505-1511.

[4] A.J. Drehman, A.L. Greer, D. Turnbull, Appl. Phys. Letts. 41 (1982) 716-717.

[5] H.S. Chen, Rep. Prog. Phys. 43 (1980) 353-432.

[6] A. Inoue, T. Zhang, T. Masumoto, Mater. Trans. JIM 30 (1989) 965-972.

[7] A. Inoue, T. Nakamura, N. Nishiyama, T. Masumoto, Mater. Trans. JIM 33 (1992) 937-945.

[8] A. Inoue, T. Zhang, N. Nishiyama, K. Ohba, T. Masumoto, Mater. Trans. JIM 34 (1993) 1234-1237.

[9] A. Inoue, T. Zhang, Mater. Trans. JIM 36 (1995) 1184-1187.

[10] A. Inoue, N. Nishiyama, H. Kimura, Mater. Trans. JIM 38 (1997) 179-183.

[11] A. Peker, W.L. Johnson, Appl. Phys. Lett. 63 (1993) 2342-2344.

[12] N. Nishiyama, K. Takenaka, H. Miura, N. Saidoh, Y. Zeng, A. Inoue, Intermetallics 30 (2012) 19-24.

[13] H.W. Kui, A.L. Greer, D. Turnbull, Appl. Phys. Lett. 45 (1984) 615-616.

[14] H.B. Lou, X.D. Wang, F. Xu, S.Q. Ding, Q.P. Cao, K. Hono, J.Z. Jiang, Appl. Phys. Lett. 99 (2011) 051910.

[15] D.H. Xu, G. Duan, W.L. Johnson, Phys. Rev. Lett. 92 (2004) 245504.

[16] P. Jia, H. Guo, Y. Li, J. Xu, E. Ma, Scripta Mater. 54 (2006) 2165-2168.

[17] F.Q. Guo, S.J. Poon, G.J. Shiflet, Appl. Phys. Lett. 83 (2003) 2575-2577.

[18] Y. Zhang, H. Tan, Y. Li, Mater. Sci. Eng. A 375-377 (2004) 436-439.

[19] R. Li, S.J. Pang, C.L. Ma, T. Zhang, Acta Mater. 55 (2007) 3719-3726.

[20] A. Inoue, T. Zhang, Mater. Sci. Eng. A 226-228 (1997) 393-396.

[21] H. Ma, L.L. Shi, J. Xu, Y. Li, E. Ma, Appl. Phys. Lett. 87 (2005) 181915.

[22] Q. Zheng, J. Xu, E. Ma, J. Appl. Phys. 102 (2007) 113519.

[23] V. Ponnambalam, S.J. Poon, G.J. Shiflet, J. Mater. Res. 19 (2004) 1320-1323.

[24] Z.P. Lu, C.T. Liu, J.R. Thompson, W.D. Porter, Phys. Rev. Lett. 92 (2004) 245503.

[25] J. Shen, Q.J. Chen, J.F. Sun, H.B. Fan, G. Wang, Appl. Phys. Lett. 86 (2005) 151907.

[26] H. Men, S.J. Pang, T. Zhang, J. Mater. Res. 21 (2006) 958-961.

[27] F.Q. Guo, H.J. Wang, S.J. Poon, G.J. Shiflet, Appl. Phys. Lett. 86 (2005) 091907.

[28] P. Gong, K.F. Yao, X. Wang, Y. Shao, Adv. Eng. Mater. 15 (2013) 691-696.

[29] M.Q. Tang, H.F. Zhang, Z.W. Zhu, H.M. Fu, A.M. Wang, H. Li, Z.Q. Hu, J. Mater. Sci. Technol. 26 (2010) 481-486.

[30] E.S. Park, D.H. Kim, J. Mater. Res. 19 (2004) 685-688.

[31] O.N. Senkov, J.M. Scott, D.B. Miracle, J. Alloys Compds. 424 (2006) 394-399.

[32] Y. Zeng, N. Nishiyama, T. Yamamoto, A. Inoue, Mater. Trans. JIM 50 (2009) 2441-2445.

[33] J. Schroers, W.L. Johnson, Appl. Phys. Lett. 84 (2004) 3666-3668. 
[34] Q.K. Jiang, G.Q. Zhang, L. Yang, X.D. Wang, K. Saksl, H. Franz, R. Wunderlich, H. Fecht, J.Z. Jiang, Acta Mater. 55 (2007) 4409-4418.

[35] M.W. Chen, Annu. Rev. Mater. Res. 38 (2008) 445-469.

[36] Y. Zhang, A.L. Greer, Appl. Phys. Lett. 89 (2006) 071907.

[37] H. Choi-Yim, W.L. Johnson, Appl. Phys. Lett. 71 (1997) 3808-3810.

[38] H. Choi-Yim, Ph.D Thesis California Institute of Technology (1999).

[39] R.D. Conner, H. Choi-Yim, W.L. Johnson, J. Mater. Res. 14 (1999) 3292-3297.

[40] H. Choi-Yim, R.D. Conner, F. Szuecs, W.L. Johnson, Acta Mater. 50 (2002) 2737-2745.

[41] Y.K. Xu, H. Ma, J. Xu, E. Ma, Acta Mater. 53 (2005) 1857-1866.

[42] T. Liu, P. Shen, F. Qiu, T. Zhang, Q. Jiang, Adv. Eng. Mater. 11 (2009) 392-398.

[43] C. Chen, Y. Xue, L. Wang, X. Cheng, F. Wang, Z. Wang, H. Zhang, A. Wang, Adv. Eng. Mater. 14 (2012) 439-444.

[44] Y.F. Xue, H.N. Cai, L. Wang, F.C. Wang, H.F. Zhang, Appl. Phys. Lett. 90 (2007) 081901.

[45] J.S.C. Jang, J.Y. Ciou, T.H. Hung, J.C. Huang, X.H. Du, Appl. Phys. Lett. 92 (2008) 011930.

[46] J.S.C. Jang, J.Y. Ciou, T.H. Li, J.C. Huang, T.G. Nieh, Intermetallics 18 (2010) 451-458.

[47] Y. Sun, H.F. Zhang, A.M. Wang, H.M. Fu, Z.Q. Hu, C.E. Wen, P.D. Hodgson, Appl. Phys. Lett. 95 (2009) 171910.

[48] M. Kinaka, H. Kato, M. Hasegawa, A. Inoue, Mater. Sci. Eng. A 494 (2008) 299.

[49] Y.L. Chen, A.M. Wang, H.M. Fu, Z.W. Zhu, H.F. Zhang, Z.Q. Hu, L. Wang, H.W. Cheng, Mater. Sci. Eng. A 530 (2011) 15-20.

[50] C.-Y. Son, C.K. Kim, S.Y. Shin, S. Lee, Mater. Sci. Eng. A 508 (2009) 15-22.

[51] Z.H. Chu, H. Kato, G.Q. Xie, G.Y. Yuan, W.J. Ding, Metall. Mater. Trans. A 44 (2013) 4595-4600.

[52] S.V. Madge, P. Sharma, D.V. Louzguine-Luzgin, A.L. Greer, A. Inoue, Scripta Mater. 62 (2010) 210-213.

[53] M.E. Siegrist, J.F. Löffler, Scripta Mater. 56 (2007) 1079-1082.

[54] S.J. Cytron, J. Mater. Sci. Lett. 1 (1982) 211-213.

[55] S. Scudino, G. Liu, K.G. Prashanth, B. Bartusch, K.B. Surreddi, B.S. Murty, J. Eckert, Acta Mater. 57 (2009) 2029-2039.

[56] R.B. Dandliker, R.D. Conner, W.L. Johnson, J. Mater. Res. 13 (1998) 2896-2901.

[57] R.D. Conner, R.B. Dandliker, W.L. Johnson, Acta Mater. 46 (1998) 6089-6102.

[58] H. Choi-Yim, R.D. Conner, F. Szuecs, W.L. Johnson, Scripta Mater. 45 (2001) 1039-1045.

[59] B. Hou, Y.-L. Li, L.-Q. Xing, C.-S. Chen, H.-C. Kou, J.-S. Li, Phil. Mag. Lett. 87 (2007) 595-601.

[60] H.F. Zhang, H. Li, A.M. Wang, H.M. Fu, B.Z. Ding, Z.Q. Hu, Intermetallics 17 (2009) 1070-1077.

[61] K. Lee, C.-Y. Son, S.-B. Lee, S.-K. Lee, S. Lee, Mater. Sci. Eng. A 527 (2010) 941-946. 
[62] C.-Y. Son, G.S. Kim, S.-B. Lee, S.-K. Lee, H.S. Kim, H. Huh, S. Lee, Metall. Mater. Trans. A 43 (2012) 4088-4096.

[63] N. Khademian, R. Gholamipour, Mater. Sci. Eng. A 527 (2010) 3079-3084.

[64] P. Wadhwa, J. Heinrich, R. Busch, Scripta Mater. 56 (2007) 73-76.

[65] Y. Kim, S.Y. Shin, J.S. Kim, H. Huh, K.J. Kim, S. Lee, Metall. Mater. Trans. A 43 (2012) 3023-3033.

[66] G.S. Kim, S.-B. Lee, S.-K. Lee, H.S. Kim, S. Lee, Metall. Mater. Trans. A 45 (2014) 1285-1293.

[67] K. Wang, T. Fujita, M.W. Chen, T.G. Nieh, H. Okada, K. Koyama, W. Zhang, A. Inoue, Appl. Phys. Lett. 91 (2007) 154101.

[68] X. Hui, J. Yu, M. Wang, W. Dong, G. Chen, Intermetallics 14 (2006) 931-935.

[69] J.W. Qiao, J. Mater. Sci. Technol. 29 (2013) 685-701.

[70] X.L. Fu, Y. Li, C.A. Schuh, Acta Mater. 55 (2007) 3059-3071.

[71] G. Chen, H. Bei, Y. Cao, A. Gali, C.T. Liu, E.P. George, Appl. Phys. Lett. 95 (2009) 081908.

[72] X.H. Du, J.C. Huang, K.C. Hsieh, Y.H. Lai, H.M. Chen, J.S.C. Jang, P.K. Liaw, Appl. Phys. Lett. 91 (2007) 131901.

[73] B.J. Park, H.J. Chang, D.H. Kim, W.T. Kim, K. Chattopadhyay, T.A. Abinandanan, S. Bhattacharyya, Phys. Rev. Lett. 96 (2006) 245503.

[74] C. Fan, C. Li, A. Inoue, Phys. Rev. B 61 (2000) 3761-3763.

[75] C. Fan, A. Inoue, Appl. Phys. Lett. 77 (2000) 46-48.

[76] L.Q. Xing, J. Eckert, W. Löser, L. Schultz, Appl. Phys. Lett. 74 (1999) 664-666.

[77] Y.C. Kim, J.H. Na, J.M. Park, D.H. Kim, J.K. Lee, W.T. Kim, Appl. Phys. Lett. 83 (2003) 3093-3095.

[78] A. Inoue, W. Zhang, T. Tsurui, A.R. Yavari, A.L. Greer, Phil. Mag. Lett. 85 (2005) 221-229.

[79] M.W. Chen, A. Inoue, C. Fan, A. Sakai, T. Sakurai, Appl. Phys. Lett. 74 (1999) 2131-2133.

[80] K. Hajlaoui, A.R. Yavari, B. Doisneau, A. LeMoulec, W.J.B. F., G. Vaughan, A.L. Greer, A. Inoue, W. Zhang, Å. Kvick, Scripta Mater. 54 (2006) 1829-1834.

[81] Y.F. Shi, M.L. Falk, Acta Mater. 56 (2008) 995-1000.

[82] A. Inoue, C. Fan, J. Saida, T. Zhang, Sci. Technol. Adv. Mater. 2 (2000) 73-76.

[83] K.F. Yao, F. Ruan, Y.Q. Yang, N. Chen, Appl. Phys. Lett. 88 (2006) 122106.

[84] Y.H. Liu, G. Wang, R.J. Wang, D.Q. Zhao, M.X. Pan, W.H. Wang, Science 315 (2007) 1385-1388.

[85] D. Sopu, Y. Ritter, H. Gleiter, K. Albe, Phys. Rev. B 83 (2011) 100202.

[86] C.C. Hays, C.P. Kim, W.L. Johnson, Phys. Rev. Lett. 84 (2000) 2901-2904.

[87] S.Y. Lee, C.P. Kim, J.D. Almer, U. Lienert, E. Ustundag, W.L. Johnson, J. Mater. Res. 22 (2007) 538-543.

[88] J.W. Qiao, Y. Zhang, G.L. Chen, Mater. Des. 30 (2009) 3966-3971.

[89] F. Szuecs, C.P. Kim, W.L. Johnson, Acta Mater. 49 (2001) 1507-1513.

[90] J.W. Qiao, Y. Zhang, P. Feng, Q.M. Zhang, G.L. Chen, Mater. Sci. Eng. A 515 (2009) 141-145. 
[91] A. Bracchi, Y.-L. Huang, M. Seibt, S. Schneider, P. Thiyagarajan, J. Appl. Phys. 99 (2006) 123519.

[92] W. Löser, J. Das, A. Güth, H.-J. Klauß, C. Mickel, U. Kühn, J. Eckert, S.K. Roy, L. Schultz, Intermetallics 12 (2004) 1153-1158.

[93] F.F. Wu, Z.F. Zhang, S.X. Mao, A. Peker, J. Eckert, Phys. Rev. B 75 (2007) 134201.

[94] D.C. Hofmann, J.-Y. Suh, A. Wiest, G. Duan, M.-L. Lind, M.D. Demetriou, W.L. Johnson, Nature 451 (2008) 1085-1090.

[95] J.W. Qiao, S. Wang, Y. Zhang, P.K. Liaw, G.L. Chen, Appl. Phys. Lett. 94 (2009) 151905.

[96] S. Roberts, C. Zachrisson, H. Kozachkov, A. Ullah, A.A. Shapiro, W.L. Johnson, D.C. Hofmann, Scripta Mater. 66 (2012) 284-287.

[97] J.-L. Cheng, G. Chen, C.-T. Liu, Y. Li, Sci. Rep. 3 (2013) 2097.

[98] G. He, J. Eckert, W. Löser, L. Schultz, Nat. Mater. 2 (2003) 33-37.

[99] F.Q. Guo, S.J. Poon, G.J. Shiflet, Phil. Mag. Lett. 88 (2008) 615-622.

[100] D.C. Hofmann, J.Y. Suh, A. Wiest, M.L. Lind, M.D. Demetriou, W.L. Johnson, PNAS 105 (2008) 20136.

[101] J.W. Qiao, A.C. Sun, E.W. Huang, Y. Zhang, P.K. Liaw, C.P. Chuang, Acta Mater. 59 (2011) 4126-4137.

[102] J.W. Qiao, J.T. Zhang, F. Jiang, Y. Zhang, P.K. Liaw, Y. Ren, G.L. Chen, Mater. Sci. Eng. A 527 (2010) 7752-7756.

[103] Y.S. Oh, C.P. Kim, S. Lee, N.J. Kim, Acta Mater. 59 (2011) 7277-7286.

[104] C. Jeon, C.P. Kim, S.-H. Joo, H.S. Kim, S. Lee, Acta Mater. 61 (2013) 3012-3026.

[105] J.W. Qiao, T. Zhang, F.Q. Yang, P.K. Liaw, S. Pauly, B.S. Xu, Sci. Rep. 3 (2013) 2816.

[106] J.T. Fan, Z.F. Zhang, B.L. Shen, S.X. Mao, Scripta Mater. 59 (2008) 603-606.

[107] M. Ferry, K.J. Laws, C. White, D.M. Miskovic, K.F. Shamlaye, W. Xu, O. Biletska, MRS Commun. 3 (2013) 1-12.

[108] M.L. Lee, Y. Li, C.A. Schuh, Acta Mater. 52 (2004) 4121-4131.

[109] Y. Zhang, W. Xu, H. Tan, Y. Li, Acta Mater. 53 (2005) 2607-2616.

[110] Z.P. Chen, J.E. Gao, Y. Wu, H. Wang, X.J. Liu, Z.P. Lu, Sci. Rep. 3 (2013) 3353.

[111] S.F. Guo, L. Liu, N. Li, Y. Li, Scripta Mater. 62 (2010) 329-332.

[112] J.E. Gao, Z.P. Chen, Q. Du, H.X. Li, Y. Wu, H. Wang, X.J. Liu, Z.P. Lu, Acta Mater. 61 (2013) 3214-3223.

[113] C. Fan, R.T. Ott, T.C. Hufnagel, Appl. Phys. Lett. 81 (2002) 1020-1022.

[114] J.W. Qiao, Y. Zhang, Intermetallics 19 (2011) 149-153.

[115] D. Banerjee, J.C. Williams, Acta Mater. 61 (2013) 844-879.

[116] S. Cai, M.R. Daymond, R.A. Holt, Acta Mater. 60 (2012) 3355-3369.

[117] G. He, Z.F. Zhang, W. Löser, J. Eckert, L. Schultz, Acta Mater. 51 (2003) 2383-2395.

[118] Z. Bian, H. Kato, C. Qin, W. Zhang, A. Inoue, Acta Mater. 53 (2005) 2037-2048. 
[119] Y.C. Kim, E. Fleury, J.-C. Lee, D.H. Kim, J. Mater. Res. 20 (2005) 2474-2479.

[120] R.T. Ott, F. Sansoz, T. Jiao, D. Warner, C. Fan, J.F. Molinari, K.T. Ramesh, T.C. Hufnagel, Metall. Mater. Trans. A 37 (2006) 3251-3258.

[121] C. Li, J.S.C. Jang, J.B. Li, D.J. Pan, S.R. Jian, J.C. Huang, T.G. Nieh, Intermetallics 30 (2012) 111-116.

[122] M. Militzer, Science 298 (2002) 975-976.

[123] Y.F. Sun, B.C. Wei, Y.R. Wang, W.H. Li, T.L. Cheung, C.H. Shek, Appl. Phys. Lett. 87 (2005) 051905.

[124] S. Pauly, G. Liu, G. Wang, J. Das, K.B. Kim, U. Kühn, D.H. Kim, J. Eckert, Appl. Phys. Lett. 95 (2009) 101906.

[125] J. Liu, X. Yuan, H. Zhang, H. Fu, Z. Hu, Mater. Trans. JIM 51 (2010) 1033-1037.

[126] Y. Wu, Y.H. Xiao, G.L. Chen, C.T. Liu, Z.P. Lu, Adv. Mater. 22 (2010) 2770-2773.

[127] S. Pauly, S. Gorantla, G. Wang, U. Kühn, J. Eckert, Nat. Mater. 9 (2010) 473-477.

[128] K.K. Song, S. Pauly, Y. Zhang, P. Gargarella, R. Li, N.S. Barekar, U. Kühn, M. Stoica, J. Eckert, Acta Mater. 59 (2011) 6620-6630.

[129] K.K. Song, S. Pauly, Y. Zhang, R. Li, S. Gorantla, N. Narayanan, U. Kühn, T. Gemming, J. Eckert, Acta Mater. 60 (2012) 6000-6012.

[130] Y. Wu, D.Q. Zhou, W.L. Song, H. Wang, Z.Y. Zhang, D. Ma, X.L. Wang, Z.P. Lu, Phys. Rev. Lett. 109 (2012) 245506.

[131] K.K. Song, S. Pauly, B.A. Sun, J. Tan, M. Stoica, U. Kühn, J. Eckert, AIP Adv. 3 (2013) 012116.

[132] Z.Q. Liu, G. Liu, R.T. Qu, Z.F. Zhang, S.J. Wu, T. Zhang, Scr. Rep. 4 (2014) 4167.

[133] J. Chen, Y. Zhang, J.P. He, K.F. Yao, B.C. Wei, G.L. Chen, Scripta Mater. 54 (2006) 1351-1355.

[134] K. Lu, Mater. Sci. Eng. R 16 (1996) 161-221.

[135] Y. Tanaka, Y. Himuro, R. Kainuma, Y. Sutou, T. Omori, K. Ishida, Science 327 (2010) 1488-1490.

[136] J.W. Seo, D. Schryvers, Acta Mater. 46 (1998) 1165-1175.

[137] K.K. Song, PhD Dissertation: Synthesis, microstructure, and deformation mechanisms of CuZr-based bulk metallic glass composites (2013).

[138] Z.P. Lu, C.T. Liu, Phys. Rev. Lett. 91 (2003) 115505.

[139] C.W. Nan, Prog. Mater. Sci. 37 (1993) 1-116.

[140] A. Inoue, Y. Yokoyama, K. Shinohara, T. Masumoto, Mater. Trans. JIM 35 (1994) 923-926.

[141] J.J. Wall, C. Fan, P.K. Liaw, C.T. Liu, H. Choo, Rev. Sci. Instrum. 77 (2006) 033902.

[142] G. Xie, D.V. Louzguine-Luzgin, H. Kimura, A. Inoue, F. Wakai, Appl. Phys. Lett. 92 (2008) 121907.

[143] T. Tamura, K. Amiya, R. Rachmat, Y. Mizutani, K. Miwa, Nat. Mater. 4 (2005) 289-292. 
[144] G. Yang, X. Lin, F. Liu, Q. Hu, L. Ma, J. Li, W. Huang, Intermetallics 22 (2012) 110-115.

[145] K.J. Laws, B. Gun, M. Ferry, Mater. Sci. Eng. A 475 (2008) 348-354.

[146] T. Dong, B. Yang, J. He, Y. Zhang, Acta Metall. Sin. 44 (2008) 659-664.

[147] T. Zhang, X. Zhang, W. Zhang, F. Jia, A. Inoue, H. Hao, Y. Ma, Mater. Lett. 65 (2011) 2257-2260.

[148] H.W. Sheng, W.K. Luo, F.M. Alamgir, J. Bai, E. Ma, Nature 439 (2006) 419-425.

[149] D.B. Miracle, D.V. Louzguine-Luzgin, L.V. Louzguina-Luzgina, A. Inoue, Int. Mater. Rev. 55 (2010) 218-256.

[150] J.W. Qiao, A.C. Sun, E.W. Huang, Y. Zhang, P.K. Liaw, C.P. Chuang, Acta Mater. 59 (2011) 4126-4137.

[151] Y. Li, Q. Guo, J.A. Kalb, C.V. Thompson, Science 322 (2008) 1816-1819.

[152] S. Ding, Y. Liu, Y. Li, Z. Liu, S. Sohn, F.J. Walker, J. Schroers, Nat. Mater. 13 (2014) 494-500.

[153] J. Yang, D.D.L. Chung, J. Mater. Sci. 24 (1989) 3605-3612.

[154] W.B. Hillig, J. Am. Ceram. Soc. 71 (1988) 96-99.

[155] E.S. Eardley, H.M. Flower, Mater. Sci. Eng. A 359 (2003) 303-312.

[156] S.C. Tjong, Z.Y. Ma, Mater. Sci. Eng. R 29 (2000) 49-113.

[157] J. Kong, Z.-T. Ye, F. Lv, J. Alloy Compds. 478 (2009) 202-205.

[158] J.W. Qiao, Y. Zhang, Z.L. Zheng, J.P. He, B.C. Wei, J. Alloy Compds. 477 (2009) 436-439.

[159] Z. Liu, R. Li, G. Liu, W. Su, H. Wang, Y. Li, M. Shi, X. Luo, G. Wu, T. Zhang, Acta Mater. 60 (2012) 3128-3139.

[160] C. Jeon, D.J. Ha, C.P. Kim, S. Lee, Metall. Mater. Trans. A 43 (2012) 3663-3674.

[161] G.Y. Sun, G. Chen, C.T. Liu, G.L. Chen, Scripta Mater. 55 (2006) 375-378.

[162] J.W. Qiao, Y. Zhang, H.J. Yang, S.B. Sang, Int. J. Miner. Metall. Mater. 20 (2013) 386-392.

[163] A. Inoue, F.L. Kong, S.L. Zhu, E. Shalaan, F.M. Al-Marzouki, Intermetallics 58 (2015) 20-30.

[164] D.C. Hofmann, H. Kozachkov, H.E. Khalifa, J.P. Schramm, M.D. Demetriou, K.S. Vecchio, W.L. Johnson, JOM 61 (2009) 11-17.

[165] D.C. Hofmann, J. Mater. (2013) http://dx.doi.org/10.1155/2013/517904.

[166] D.C. Hofmann, J.-Y. Suh, A. Wiest, W. Johnson, Scripta Mater. 59 (2008) 684-687.

[167] W. Kurz, B. Giovanola, R. Trivedi, Acta Metall. 34 (1986) 823-830.

[168] Y. Li, S.C. Ng, Z.P. Lu, Y.P. Feng, Mater. Lett. 34 (1998) 318-321.

[169] H. Tan, Y. Zhang, X. Hu, Y.P. Feng, Y. Li, Mater. Sci. Eng. A 375-377 (2004) 407-410.

[170] H. Tan, Y. Zhang, Y. Li, Intermetallics 10 (2002) 1203-1205.

[171] J.W. Qiao, Y. Zhang, P.K. Liaw, Adv. Eng. Mater. 10 (2008) 1039-1042.

[172] J.W. Qiao, P. Feng, Y. Zhang, Q.M. Zhang, G.L. Chen, J. Alloy Compds. 486 (2009) 527-531. 
[173] J.W. Qiao, P. Feng, Y. Zhang, Q.M. Zhang, P.K. Liaw, G.L. Chen, J. Mater. Res. 25 (2010) 2264-2270.

[174] J.T. Zhang, J.W. Qiao, Y. Zhang, Acta Metall. Sin. 47 (2011) 236-240.

[175] Y.S. Wang, G.J. Hao, Y. Zhang, J.P. Lin, J.W. Qiao, Metall. Mater. Trans. A 45 (2014) 2359-2362.

[176] M.H. Cohen, D. Turnbull, J. Chem. Phys. 31 (1959) 1164-1169.

[177] D. Turnbull, M.H. Cohen, J Chem Phys 34 (1961) 120-125.

[178] F. Spaepen, Acta Metall. 25 (1977) 407-415.

[179] C.A. Schuh, A.C. Lund, Nat. Mater. 2 (2003) 449-452.

[180] J.C. Dyre, Rev. Mod. Phys. 78 (2006) 953-972.

[181] M.Q. Jiang, L.H. Dai, Phys. Rev. B 76 (2007) 054204.

[182] W.L. Johnson, J. Lu, M.D. Demetriou, Intermetallics 10 (2002) 1039-1046.

[183] Q. Yang, A. Mota, M. Ortiz, Comput. Mech. 37 (2006) 194-204.

[184] Y.F. Gao, B. Yang, T.G. Nieh, Acta Mater. 55 (2007) 2319-2327.

[185] P. Thamburaja, R. Ekambaram, J. Mech. Phys. Solids 57 (2007) 1263-1273.

[186] R. Huang, Z. Suo, J.H. Prevost, W.D. Nix, J. Mech. Phys. Solids 50 (2002) 1011-1027.

[187] M.Q. Jiang, L.H. Dai, J. Mech. Phys. Solids 57 (2009) 1267-1292.

[188] P. Thamburaja, J. Mech. Phys. Solids 59 (2011) 1552-1575.

[189] A.S. Argon, H.Y. Kuo, Mater. Sci. Eng. A 39 (1979) 101-109.

[190] A.S. Argon, Acta metal. 27 (1979) 47-58.

[191] J.D. Eshelby, Proc. R. Soc. Lond. A 241 (1957) 376-396.

[192] J.D. Eshelby, Proc. R. Soc. Lond. A 252 (1959) 561-569.

[193] F. Spaepen, Defects in amorphous metals, in: R. Balian, M. Kleman, J. Poirier (Eds.), Physics of defects, North-Hollan Press, Amsterdam, 1981, pp. 133-174.

[194] F.S.M. Heggen, M. Feuerbacher, J. Appy. Phys. 97 (2005) 033506.

[195] A. Lemaître, Phys. Rev. Lett. 89 (2002) 195503.

[196] L. Li, E.R. Homer, C.A. Schuh, Acta Mater. 61 (2013) 3347-3359.

[197] M.Q. Jiang, W.H. Wang, L.H. Dai, Scripta Mater. 60 (2009) 1004-1007.

[198] M.L. Falk, J.S. Langer, Phys. Rev. E 57 (1998) 7192.

[199] P. Schall, D.A. Weitz, F. Spaepen, Science 318 (2007) 1895-1899.

[200] M.Q. Jiang, S.Y. Jiang, L.H. Dai, Chin. Phys. Lett. 26 (2009) 106103.

[201] S. Ogata, F. Shimizu, J. Li, M. Wakeda, Y. Shibutani, Intermetallics 14 (2006) 1033-1037.

[202] J.J. Lewandowski, W.H. Wang, A.L. Greer, Philos. Mag. Lett. 85 (2005) 77-87.

[203] M.Q. Jiang, L.H. Dai, Philos. Mag. Lett. 90 (2010) 269-277.

[204] G.N. Greaves, A.L. Greer, R.S. Lakes, T. Rouxel, Nat. Mater. 10 (2011) 823-837.

[205] M.L. Falk, J.S. Langer, L. Pechenik, Phys. Rev. E 70 (2004) 011507.

[206] X.K. Xi, D.Q. Zhao, M.X. Pan, W.H. Wang, Y. Wu, J.J. Lewandowski, Phys. Rev. Lett. 94 (2005) 125501.

[207] G. Wang, D.Q. Zhao, H.Y. Bai, M.X. Pan, A.L. Xia, B.S. Han, X.K. Xi, Y. Wu, W.H. Wang, Phys. Rev. Lett. 98 (2007) 235501. 
[208] J.X. Meng, Z. Ling, M.Q. Jiang, H.S. Zhang, L.H. Dai, Appl. Phys. Lett. 92 (2008) 171909.

[209] M.Q. Jiang, Z. Ling, J.X. Meng, L.H. Dai, Philos. Mag. Lett. 88 (2008) 407-426.

[210] M.Q. Jiang, J.X. Meng, J.B. Gao, X.L. Wang, T. Rouxel, V. Keryvin, Z. Ling, L.H. Dai, Intermetallics 18 (2010) 2468-2471.

[211] F. Spaepen, Acta Metall. 23 (1975) 615-620.

[212] A.S. Argon, M.S. Salama, Mater. Sci. Eng., 23 (1976) 219-230.

[213] C.A. Pampillo, J. Mater. Sci. 10 (1975) 1194-1227.

[214] R. Raghavan, P. Murali, U. Ramamurty, Acta Mater. 57 (2009) 3332-3340.

[215] J.P. Escobedo, Y.M. Gupta, J. Appl. Phys. 107 (2010) 123502.

[216] G. Li, M.Q. Jiang, F. Jiang, L. He, J. Sun, Appl. Phys. Lett. 102 (2013) 171901.

[217] M.Q. Jiang, Z. Ling, J.X. Meng, J.B. Gao, L.H. Dai, Scripta Mater. 62 (2010) 572-575.

[218] F. Jiang, M.Q. Jiang, H.F. Wang, Y.L. Zhao, L. He, J. Sun, Acta Mater. 59 (2011) 2057-2068.

[219] G. Wang, K.C. Chan, X.H. Xu, W.H. Wang, Acta Mater. 56 (2008) 5845-5860.

[220] P. Murali, T.F. Guo, Y.W. Zhang, R. Narasimhan, Y. Li, H.J. Gao, Phy. Rev. Lett. 107 (2011) 215501.

[221] F.H. Stillinger, T.A. Weber, J. Chem. Phys. 88 (1988) 7818-7825.

[222] P.G. Debenedetti, F.H. Stillinger, Nature 410 (2001) 259-267.

[223] J.P.K. Doye, D.J. Wales, J. Chem. Phys. 116 (2002) 3777-3788.

[224] D.J. Wales, Science 293 (2001) 2067-2070.

[225] D.L. Malandro, D.J. Lacks, J. Chem. Phys. 110 (1999) 4593-4601.

[226] W.L. Johnson, K. Samwer, Phys. Rev. Lett. 95 (2005) 195501.

[227] M.D. Demetriou, J.S. Harmon, M. Tao, G. Duan, K. Samwer, W.L. Johnson, Phys. Rev. Lett. 97 (2006) 065502.

[228] D. Pan, A. Inoue, T. Sakurai, M.W. Chen, PNAS 105 (2008) 14769.

[229] S.G. Mayr, Phys. Rev. Lett. 97 (2006) 195501.

[230] W. Jiao, P. Wen, H.L. Peng, H.Y. Bai, B.A. Sun, W.H. Wang, Appl. Phys. Lett. 102 (2013) 101903.

[231] W. Jiao, B.A. Sun, P. Wen, H.Y. Bai, Q.P. Kong, W.H. Wang, Appl. Phys. Lett. 103 (2013) 081904.

[232] Z.G. Zhu, P. Wen, D.P. Wang, R.J. Xue, D.Q. Zhao, W.H. Wang, J. Appl. Phys. 114 (2013) 083512.

[233] H.B. Yu, X. Shen, Z. Wang, L. Gu, W.H. Wang, H.Y. Bai, Phys. Rev. Lett. 108 (2012) 015504.

[234] Z. Lu, W. Jiao, W.H. Wang, H.Y. Bai, Phys. Rev. Lett. 113 (2014) 045501.

[235] Z. Wang, B.A. Sun, H.Y. Bai, W.H. Wang, Nat. Commun. 5 (2014) 5823.

[236] J.C. Qiao, J.M. Pelletier, J. Mater. Sci. Technol. 30 (2014) 523-545.

[237] J.C. Qiao, Y.J. Wang, J.M. Pelletier, L.M. Keer, M.E. Fine, Y. Yao, Acta Mater. 98 (2015) 43-50.

[238] W.H. Wang, Y. Yang, T.G. Nieh, C.T. Liu, Intermetallics 67 (2015) 81-86. 
[239] A. Portevin, F.L. Chatelier, C R Acad Sci Paris 176 (1923) 507-510.

[240] M.A. Lebyodkin, Y. Brechet, Y. Estrin, L.P. Kubin, Phys. Rev. Lett. 74 (1995) 4758-4761.

[241] A. Benallal, T. Berstad, T. Børvik, O.S. Hopperstad, I. Koutiri, R.N.d. Codes, Int. J. Plast. 24 (2008) 1916-1945.

[242] R.A. Mulford, U.F. Kocks, Acta Metall. 27 (1979) 1125-1134.

[243] G. Wang, K.C. Chan, L. Xia, P. Yu, J. Shen, W.H. Wang, Acta Mater. 57 (2009) 6146-6155.

[244] B.A. Sun, H.B. Yu, W. Jiao, H.Y. Bai, D.Q. Zhao, W.H. Wang, Phys. Rev. Lett. 105 (2010) 035501.

[245] S.X. Song, T.G. Nieh, Intermetallics 19 (2011) 1968-1977.

[246] R. Maaß, D. Klaumünzer, J.F. Löffler, Acta Mater. 59 (2011) 3205-3211.

[247] J.W. Qiao, Y. Zhang, P.K. Liaw, Intermetallics 18 (2010) 2057-2064.

[248] J.W. Qiao, F.Q. Yang, G.Y. Wang, P.K. Liaw, Y. Zhang, Scripta Mater. 63 (2010) 1081-1084.

[249] B.A. Sun, S. Pauly, J. Tan, M. Stoica, W.H. Wang, U. Kühn, J. Eckert, Acta Mater. 60 (2012) 4160-4171.

[250] J.L. Ren, C. Chen, Z.Y. Liu, R. Li, G. Wang, Phys. Rev. B 86 (2012) 134303.

[251] J. Antonaglia, X. Xie, G. Schwarz, M. Wraith, J.W. Qiao, Y. Zhang, P.K. Liaw, J.T. Uhl, K.A. Dahmen, Scr. Rep. 4 (2014) 4382.

[252] W.H. Jiang, G.J. Fan, F.X. Liu, G.Y. Wang, H. Choo, P.K. Liaw, Int. J. Plast. 24 (2008) 1-16.

[253] Z. Wang, J.W. Qiao, H.J. Yang, P.K. Liaw, C.J. Huang, L.F. Li, Metall. Mater. Trans. A 46 (2015) 2404-2414.

[254] B.A. Sun, S. Pauly, J. Hu, W.H. Wang, U. Kühn, J. Eckert, Phys. Rev. Lett. 110 (2013) 225501.

[255] D. Klaumünzer, R. Maaß, J.F. Löffler, J. Mater. Res. 26 (2011) 1453-1463.

[256] Y.Q. Cheng, Z. Han, Y. Li, E. Ma, Phys. Rev. B 80 (2009) 134115.

[257] A.L. Greer, Y.Q. Cheng, E. Ma, Mater. Sci. Eng. R 74 (2013) 71-132.

[258] Z. Han, W.F. Wu, Y. Li, Y.J. Wei, H.J. Gao, Acta Mater. 57 (2009) 1367-1372.

[259] J.J. Kim, Y. Choi, S. Suresh, A.S. Argon, Science 295 (2002) 654-657.

[260] J.J. Lewandowski, A.L. Greer, Nat. Mater. 5 (2006) 15-18.

[261] P. Thurnheer, R. Maaß, S. Pogatscher, J.F. Löffler, Appl. Phys. Lett. 104 (2014) 101910.

[262] J.W. Qiao, Z. Wang, H.J. Yang, M. Li, W. Liang, B.S. Xu, AIP Adv. 3 (2013) 032105.

[263] J.W. Qiao, Z. Wang, Z.M. Jiao, H.J. Yang, S.G. Ma, Z.H. Wang, B.S. Xu, Mater. Sci. Eng. A 609 (2014) 222-225.

[264] Y.J. Huang, J. Shen, J.F. Sun, Appl. Phys. Lett. 90 (2007) 081919.

[265] J.R. Greer, J.T.M.D. Hosson, Prog. Mater. Sci. 56 (2011) 654-724.

[266] F. Berto, P. Lazzarin, Mater. Sci. Eng. R 75 (2014) 1-48.

[267] B. Zhang, D.Q. Zhao, M.X. Pan, W.H. Wang, A.L. Greer, Phys. Rev. Lett. 94 (2005) 205502. 
[268] A. Inoue, B. Shen, H. Koshiba, H. Kato, A.R. Yavari, Nat. Mater. 2 (2003) 661-663.

[269] B. Yang, C.T. Liu, T.G. Nieh, Appl. Phys. Lett. 88 (2006) 221911.

[270] C.R. Cao, D.W. Ding, D.Q. Zhao, E. Axinte, H.Y. Bai, W.H. Wang, Mater. Des. 60 (2014) 576-579.

[271] B.J. Alder, T.E. Wainwright, J. Chem. Phys. 27 (1957) 1208-1209.

[272] W.W. Wood, J.D. Jacobson, J. Chem. Phys. 27 (1957) 1207-1208.

[273] S. Takeuchi, K. Edagawa, Prog. Mater Sci. 56 (2011) 785-816.

[274] M.M. Trexler, N.N. Thadhani, Prog. Mater. Sci. 55 (2010) 759-839.

[275] J.W. Qiao, H.L. Jia, Y. Zhang, P.K. Liaw, L.F. Li, Mater. Chem. Phys. 136 (2012) 75-79.

[276] Y. Zhang, W.H. Wang, A.L. Greer, Nat. Mater. 5 (2006) 857-860.

[277] W.H. Jiang, G.J. Fan, H. Choo, P.K. Liaw, Mater. Lett. 60 (2006) 3537-3540.

[278] L.Y. Chen, Q. Ge, S. Qu, Q.K. Jiang, X.P. Xie, J.Z. Jiang, Appl. Phys. Lett. 92 (2008) 211905.

[279] M.D. Demetriou, C. Veazey, J.S. Harmon, J.P. Schramm, W.L. Johnson, Phys. Rev. Lett. 101 (2008) 145702.

[280] J.X. Zhao, F.F. Wu, R.T. Qu, S.X. Li, Z.F. Zhang, Acta Mater. 58 (2010) 5420-5432.

[281] R.T. Qu, M. Calin, J. Eckert, Z.F. Zhang, Scripta Mater. 66 (2012) 733-736.

[282] Z.T. Wang, J. Pan, Y. Li, C.A. Schuh, Phys. Rev. Lett. 111 (2013) 135504.

[283] Q. Wang, Y. Yang, H. Jiang, C.T. Liu, H.H. Ruan, J. Lu, Sci. Rep. 4 (2014) 4757.

[284] D. Jang, J.R. Greer, Nat. Mater. 9 (2010) 215-219.

[285] H. Guo, P.F. Yan, Y.B. Wang, J. Tan, Z.F. Zhang, M.L. Sui, E. Ma, Nat. Mater. 6 (2007) 735-739.

[286] J.H. Luo, F.F. Wu, J.Y. Huang, J.Q. Wang, S.X. Mao, Phys. Rev. Lett. 104 (2010) 215503.

[287] D.J. Magagnosc, R. Ehrbar, G. Kumar, M.R. He, J. Schroers, D.S. Gianola, Scr. Rep. 3 (2013) 1096.

[288] X.L. Lu, Q.H. Lu, Y. Li, L. Lu, Scr. Rep. 3 (2013) 3319.

[289] B. Sarac, J. Schroers, Nat. Commus. 4 (2013) 2058.

[290] N.J. Petch, J. Iron Steel Inst. 174 (1953) 25-28.

[291] J.R. Greer, J.T.M. De Hosson, Prog. Mater Sci. 56 (2011) 654-724.

[292] J.C. Ye, J. Lu, Y. Yang, P.K. Liaw, Acta Mater. 57 (2009) 6037-6046.

[293] X.W. Gu, M. Jafary-Zadeh, D.Z. Chen, Z. Wu, Y.-W. Zhang, D.J. Srolovitz, J.R. Greer, Nano Lett. 14 (2014) 5858-5864.

[294] D.C. Jang, C.T. Gross, J.R. Greer, Int. J. Plast. 27 (2011) 858-867.

[295] H.B. Ke, B.A. Sun, C.T. Liu, Y. Yang, Acta Mater. 63 (2014) 180-190.

[296] H. Zhang, Z.F. Zhang, Z.G. Wang, K.Q. Qiu, H.F. Zhang, Q.S. Zang, Metall. Mater. Trans. A 37 (2006) 2459-2469.

[297] Z.F. Zhang, G. He, J. Eckert, L. Schultz, Phys. Rev. Lett. 91 (2003) 045505.

[298] B.Y. Zhang, X.H. Chen, S.S. Wang, D.Y. Lin, X.D. Hui, Mater. Lett. 93 (2013) 210-214. 
[299] J. Mu, Z. Zhu, R. Su, Y. Wang, H. Zhang, Y. Ren, Acta Mater. 61 (2013) 5008-5017.

[300] J.-C. Lee, Y.C. Kim, J.-P. Ahn, S. Lee, B.-J. Lee, Appl. Phys. Lett. 84 (2004) 2781-2783.

[301] L. Anand, C. Su, Acta Mater. 55 (2007) 3735-3747.

[302] P.S. Singh, R.L. Narayan, I. Sen, D.C. Hoffmann, U. Ramamurty, Mater. Sci. Eng. A 534 (2012) 476-484.

[303] X.L. Fu, Y. Li, C.A. Schuh, J. Mater. Res. 22 (2007) 1564-1573.

[304] H. Li, C. Fan, K. Tao, H. Choo, P.K. Liaw, Adv. Mater. 18 (2006) 752-754.

[305] E.D. Tabachnikova, A.V. Podolskii, V.Z. Bengus, S.N. Smirnor, D.V. Luzgin, A. Inoue, Low Temp. Phys. 34 (2008) 675-677.

[306] J.W. Qiao, H.L. Jia, C.P. Chuang, E.W. Huang, G.Y. Wang, P.K. Liaw, Y. Ren, Y. Zhang, Scripta Mater. 63 (2010) 871-874.

[307] C. Fan, H. Li, L.J. Kecskes, K. Tao, H. Choo, P.K. Liaw, C.T. Liu, Phys. Rev. Lett. 96 (2006) 145506.

[308] J.W. Qiao, P.K. Liaw, Y. Zhang, Scripta Mater. 64 (2011) 462-465.

[309] H. Choi-Yim, S.-Y. Lee, R.D. Conner, Scripta Mater. 58 (2008) 763-766.

[310] X. Chen, B. Zhang, G. Chen, Y. Zhang, X. Hui, Z. Lu, X. Liu, Y. Xu, X. Xing, Intermetallics 18 (2010) 2034-2038.

[311] D.J. Ha, C.P. Kim, S. Lee, Mater. Sci. Eng. A 552 (2012) 404-409.

[312] D.J. Ha, C.P. Kim, S. Lee, Mater. Sci. Eng. A 558 (2014) 558-565.

[313] Z. Zhu, H. Zhang, Z. Hu, W. Zhang, A. Inoue, Scripta Mater. 62 (2010) 278-281.

[314] T.G. Nieh, J. Wadsworth, C.T. Liu, T. Ohkubo, Y. Hirotsu, Acta Mater. 49 (2001) 2887-2896.

[315] W.J. Kim, D.S. Ma, H.G. Jeong, Scripta Mater. 49 (2003) 1067-1073.

[316] N. Li, X. Xu, Z. Zheng, L. Liu, Acta Mater. 65 (2014) 400-411.

[317] H.M. Fu, N. Liu, A.M. Wang, H. Li, Z.W. Zhu, H.W. Zhang, H.F. Zhang, Z.Q. Hu, Mater. Des. 58 (2014) 182-186.

[318] J.W. Qiao, Y. Zhang, H.L. Jia, H.J. Yang, P.K. Liaw, B.s. Xu, Appl. Phys. Lett. 100 (2012) 121902.

[319] S.F. Guo, K.C. Chan, Q. Chen, J.J. Li, L. Liu, Scripta Mater. 60 (2009) 369-372.

[320] K.C. Chan, Q. Chen, L. Liu, Intermetallics 15 (2007) 500-505.

[321] K. Marandi, P. Thamburaja, V.P.W. Shim, Mech. Mater. 75 (2014) 151-164.

[322] J.W. Qiao, Unpublished data.

[323] J. Bai, H.C. Kou, J. Wang, J.S. Li, R. Hu, Mater. Lett. 117 (2014) 228-230.

[324] R.L. Narayan, P.S. Singh, D.C. Hofmann, N. Hutchinson, K.M. Flores, U. Ramamurty, Acta Mater. 60 (2012) 5089-5100.

[325] G.P. Zheng, Y. Shen, J. Alloys Compd. 509 (2011) S136-S140.

[326] F. Abdeljawad, M. Fontus, M. Haataja, Appl. Phys. Lett. 98 (2011) 031909.

[327] H. Zhou, S. Qu, W. Yang, Int. J. Plast. 44 (2013) 147-160.

[328] T.J. Hardin, E.R. Homer, Acta Mater. 83 (2015) 203-215.

[329] A.S. Khan, Y.S. Suh, R. Kazmi, Int. J. Plast. 20 (2004) 2233-2248. 
[330] G.B. Chai, P. Manikandan, Compo. Struct. 107 (2014) 363-381.

[331] T.C. Hufnagel, T. Jiao, Y. Li, L.Q. Xing, K.T. Ramesh, J. Mater. Res. 17 (2002) 1441-1445.

[332] H.A. Bruck, A.J. Rosakis, W.L. Johnson, J. Mater. Res. 11 (2006) 503-511.

[333] F. Yuan, V. Prakash, J.J. Lewandowski, J. Mater. Res. 22 (2007) 402-411.

[334] G. Sunny, F. Yuan, V. Prakash, J. Lewandowski, J. Appl. Phys. 104 (2008) 093522.

[335] J. Liu, V.P.W. Shim, Int. J. Impact Eng. 60 (2013) 37-43.

[336] F. Xi, Y. Yu, C. Dai, Y. Zang, L. Cai, J. Appl. Phys. 108 (2010) 083537.

[337] R.W. Armstrong, S.M. Walley, Int. Mater. Rev. 53 (2008) 105-128.

[338] H. Li, G. Subhash, L.J. Kecskes, R.J. Dowding, Mater. Sci. Eng. A 403 (2005) 134-143.

[339] Y.F. Xue, H.N. Cai, L. Wang, F.C. Wang, H.F. Zhang, Mater. Sci. Eng. A 445-446 (2007) 275-280.

[340] Y.F. Xue, H.N. Cai, L. Wang, F.C. Wang, H.F. Zhang, Z.Q. Hu, Comp. Sci. Tech. 68 (2008) 3396-3400.

[341] C.-Y. Son, G.S. Kim, S.-B. Lee, S.-K. Lee, H.S. Kim, H. Huh, S. Lee, Metall. Mater. Trans. A 43 (2012) 1911-1920.

[342] M. Martin, L. Meyer, L. Kecskes, N.N. Thadhani, J. Mater. Res. 24 (2009) 66-78.

[343] K.Q. Qiu, A.M. Wang, H.F. Zhang, B.Z. Ding, Z.Q. Hu, Intermetallics 10 (2002) 1283-1288.

[344] D.-G. Lee, Y.G. Kim, S. Lee, N.J. Kim, Metall. Mater. Trans. A 37 (2006) 2893-2897.

[345] J.H. Chen, M.Q. Jiang, Y. Chen, L.H. Dai, Mater. Sci. Eng. A 576 (2014) 134-139.

[346] J.W. Qiao, H.Y. Ye, Y.S. Wang, S. Pauly, H.J. Yang, Z.H. Wang, Mater. Sci. Eng. A 585 (2013) 277-280.

[347] C. Jeon, M. Kang, C.P. Kim, H.S. Kim, S. Lee, Mater. Sci. Eng. A 579 (2013) 77-58.

[348] J.W. Qiao, M.Y. Chu, L. Cheng, H.Y. Ye, H.J. Yang, S.G. Ma, Z.H. Wang, Mater. Lett. 119 (2014) 92-95.

[349] G.R. Johnson, W. Cook, Eng. Fract. Mech. 21 (1985) 31-48.

[350] J.W. Qiao, M.M. Meng, Z.H. Wang, C.J. Huang, R. Li, Y.S. Wang, H.J. Yang, Y. Zhang, L.F. Li, AIP Adv. 4 (2014) 117107.

[351] F.F. Wu, Z.F. Zhang, A. Peker, S.X. Mao, J. Das, J. Eckert, J. Mater. Res. 21 (2006) 2331-2336.

[352] T. Mukai, T.G. Nieh, Y. Kawamura, A. Inoue, K. Higashi, Scripta Mater. 46 (2002) 43-47.

[353] Y.F. Xue, L. Wang, H.W. Cheng, F.C. Wang, H.F. Zhang, A.M. Wang, J. Mater. Sci. Technol. 26 (2010) 908-913.

[354] C.P. Koh, V.P.W. Shim, V.B.C. Tan, B.L. Tan, Int. J. Impact Eng. 35 (2008) 559-568.

[355] F. Yang, V. Prakash, J.J. Lewandowski, Mech. Mater. 41 (2009) 886-897. 
[356] W. Zheng, Y.J. Huang, B.J. Pang, J. Shen, Mater. Sci. Eng. A 529 (2011) 352-360.

[357] R.D. Conner, R.B. Dandliker, V. Scruggs, W.L. Johnson, Int. J. Impact Eng. 24 (2000) 435-444.

[358] Z. Wu, C.-M. Yang, Q.-J. Liu, Y. Ma, Rare Met. 33 (2014) 330-336.

[359] X.W. Chen, L.M. Wei, J.C. Li, Int. J. Impact Eng. 79 (2015) 102-116.

[360] S. Zhuang, J. Lu, G. Ravichandran, Appl. Phys. Lett. 80 (2002) 4522-4544.

[361] L. Hamill, S. Roberts, M. Davidson, W.L. Johnson, S. Nutt, D.C. Hofmann, Adv. Eng. Mater. 16 (2014) 85-93.

[362] P. Tandaiya, U. Ramamurty, R. Narasimhan, J. Mech. Phys. Solids 57 (2009) 1880-1897.

[363] K.M. Flores, R.H. Dauskardt, J. Mech. Phys. Solids 54 (2006) 2418-2435.

[364] J. Yi, S.M. Seifi, W.H. Wang, J.J. Lewandowski, J. Mater. Sci. Technol. 30 (2014) 627-630.

[365] H. Kimura, T. Masumoto, Scripta Metall. 9 (1975) 211-221.

[366] V. Ocelik, P. Diko, V. Hajko, J. Miskuf, P. Duhaj, Kovove Materialy-Metallic Materials 24 (1986) 457-466.

[367] V. Ocelik, P. Diko, V. Hajko, J. Miskuf, P. Duhaj, J. Mater. Sci. 22 (1987) 2305-2308.

[368] A.T. Alpas, L. Edwards, C.N. Reid, Metall. Trans. A 20 (1989) 1395-1409.

[369] L.A. Davis, J. Mater. Sci. 10 (1975) 1557-1564.

[370] V. Ocelik, P. Diko, K. Csach, V. Hajko, V.Z. Bengus, E.D. Tabachnikova, E.B. Korolkova, P. Duhaj, J. Mater. Sci. 22 (1987) 3732-3736.

[371] M. Demetriou, M. Launey, G. Garrett, J. Schramm, D. Hofmann, W. Johnson, R. Ritchie, Nat. Mater. 10 (2011) 123-128.

[372] C.J. Gilbert, R.O. Ritchie, W.L. Johnson, Appl. Phys. Lett. 71 (1997) 476.

[373] A. Kawashima, H. Kurishita, H. Kimura, T. Zhang, A. Inoue, Mater. Trans. 46 (2005) 1725-1732.

[374] K. Fujita, A. Okamoto, N. Nishiyama, Y. Yokoyama, H. Kimura, A. Inoue, J. Alloys Compd. 434-435 (2007) 22-27.

[375] X.J. Gu, S.J. Poon, G.J. Shiflet, J.J. Lewandowski, Acta Mater. 58 (2010) 1708-1720.

[376] J. Schroers, W.L. Johnson, Phys. Rev. Lett. 93 (2004) 255506.

[377] P. Wesseling, T.G. Nieh, W.H. Wang, J.J. Lewandowski, Scripta Mater. 51 (2004) 151-154.

[378] J.J. Lewandowski, A.K. Thurston, P. Lowhaphandu, Mater. Res. Soc. Symp. Proc. 754 (2002) 307-313.

[379] J. Xu, U. Ramamurty, E. Ma, JOM 62 (2010) 10-18.

[380] D.L. Henann, L. Anand, Acta Mater. 57 (2009) 6057-6074.

[381] P. Lowhaphandu, L.A. Ludrosky, S.L. Montgomery, J.J. Lewandowski, Intermetallics 8 (2000) 487-492.

[382] J.J. Lewandowski, Mater. Trans. 42 (2001) 633-637.

[383] P. Lowhaphandu, J.J. Lewandowski, Scripta Mater. 38 (1998) 1811-1817.

[384] S.G. Wang, M.Y. Sun, Z.Q. Song, J. Xu, Intermetallics 29 (2012) 123-132. 
[385] R.O. Ritchie, Science 320 (2008) 448.

[386] Q. He, Y.-Q. Cheng, E. Ma, J. Xu, Acta Mater. 59 (2011) 202-215.

[387] Q. He, J.K. Shang, E. Ma, J. Xu, Acta Mater. 60 (2012) 4940-4949.

[388] M.E. Launey, R. Busch, J.J. Kruzic, Acta Mater. 56 (2008) 500-510.

[389] J.Y. Suh, R.D. Conner, C.P. Kim, M.D. Demetriou, W.L. Johnson, J. Mater. Res. 25 (2010) 982-990.

[390] C.J. Gilbert, V. Schroeder, R.O. Ritchie, Metall. Mater. Trans. A 30A (1999) 1739-1753.

[391] K.M. Flores, R.H. Dauskardt, Scripta Mater. 41 (1999) 937-943.

[392] B. Gludovatz, S.E. Naleway, R.O. Ritchie, J.J. Kruzic, Acta Mater. 70 (2014) 198-207.

[393] V. Keryvin, Y. Nadot, Y. Yokoyama, Scripta Mater. 57 (2007) 145-148.

[394] V. Keryvin, C. Bernard, J.C. Sanglebœuf, Y. Yokoyama, T. Rouxel, J. Non-Cryst. Solids 352 (2006) 2863-2868.

[395] C. Can Aydiner, E. Üstündag, M.B. Prime, A. Peker, J. Non-Cryst. Solids 316 (2003) 82-95.

[396] C.C. Yuan, J. Ma, X.K. Xi, Mater. Sci. Eng. A 532 (2012) 430-434.

[397] M.L. Falk, Phys. Rev. B 60 (1999) 7062-7070.

[398] C.H. Rycroft, E. Bouchbinder, Phys. Rev. Lett. 109 (2012) 194301.

[399] S.F. Pugh, Philos. Mag. 45 (1954) 823-843.

[400] A. Cottrell, MRS Bull. 22 (1997) 15-19.

[401] J.J. Lewandowski, M. Shazly, A. Shamimi Nouri, Scripta Mater. 54 (2006) 337-341.

[402] P. Tandaiya, U. Ramamurty, G. Ravichandran, R. Narasimhan, Acta Mater. 56 (2008) 6077-6086.

[403] Z.-D. Zhu, P. Jia, J. Xu, Scripta Mater. 64 (2011) 785-788.

[404] Z.Q. Liu, Z.F. Zhang, J. Appl. Phys. 115 (2014) 163505.

[405] P.J. Hsieh, L.C. Yang, H.C. Su, C.C. Lu, J.S.C. Jang, J. Alloys Compd. 504, Supplement 1 (2010) S98-S101.

[406] J.S.C. Jang, T.H. Li, S.R. Jian, J.C. Huang, T.G. Nieh, Intermetallics 19 (2011) 738-743.

[407] X. Xi, D. Zhao, M. Pan, W. Wang, Y. Wu, J. Lewandowski, Phys. Rev. Lett. 94 (2005) 125510.

[408] G.P. Zheng, Y. Shen, Int. J. Solids Struct. 47 (2010) 320-329.

[409] H.A. Hassan, J.J. Lewandowski, Mater. Sci. Eng. A 586 (2013) 413-417.

[410] N. Nagendra, U. Ramamurty, T.T. Goh, Y. Li, Acta Mater. 48 (2000) 2603-2615.

[411] R. Raghavan, V.V. Shastry, A. Kumar, T. Jayakumar, U. Ramamurty, Intermetallics 17 (2009) 835-839.

[412] M.E. Launey, D.C. Hofmann, J.Y. Suh, H. Kozachkov, W.L. Johnson, R.O. Ritchie, Appl. Phys. Lett. 94 (2009) 241910.

[413] S.F. Guo, K.C. Chan, L. Liu, J. Alloys Compds. 509 (2011) 9441-9446.

[414] H.A. Hassan, A.B. El-Shabasy, J.J. Lewandowski, Mater. Sci. Eng. A 540 (2012) 97-101. 
[415] B. Gludovatz, A. Hohenwarter, D. Catoor, E.H. Chang, E.P. George, R.O. Ritchie, Science 345 (2014) 1153-1158.

[416] M.L. Sharp, G.E. Nordmark, C.C. Menzemer, Fatigue Design of Aluminum Components and Structures, McGraw-Hill Professional, 1996.

[417] J.Y. Mann, Fatigue of Materials, Melbourne University Press, Australia, 1967.

[418] T. Mukaia, T.G. Nieh, Y. Kawamura, A. Inoue, K. Higashi, Intermeallics 10 (2002) 1071.

[419] G. He, W. Löser, J. Eckert, L. Schultz, Mater. Sci. Eng. A 352 (2003) 179-185.

[420] J. Pan, Q. Chen, L. Liu, Y. Li, Acta Mater. 59 (2011) 5146.

[421] T. Ogura, T. Masumoto, K. Fukushima, Scripta Metall. 9 (1975) 109-114.

[422] L.A. Davis, J. Mater. Sci. 11 (1976) 711-717.

[423] C.J. Gilbert, J.M. Lippmann, R.O. Ritchie, Scripta Mater. 38 (1997) 537.

[424] B.C. Menzel, R.H. Dauskardt, Acta Mater. 54 (2006) 935-943.

[425] W.H. Peter, P.K. Liaw, R.A. Buchanan, C.T. Liu, C.R. Brooks, J.A. Horton, C.A. Carmichael, J.L. Wright, Intermetallics 10 (2002) 1125-1129.

[426] W.H. Peter, R.A. Buchanan, C.T. Liu, P.K. Liaw, J. Non-Cryst. Solids 317 (2003) 187-192.

[427] G.Y. Wang, P.K. Liaw, W.H. Peter, B. Yang, Y. Yokoyama, M.L. Benson, B.A. Green, M.J. Kirkham, S.A. White, T.A. Saleh, R.L. McDaniels, R.V. Steward, R.A. Buchanan, C.T. Liu, C.R. Brooks, Intermetallics 12 (2004) 885-892.

[428] G.Y. Wang, P.K. Liaw, W.H. Peter, B. Yang, M. Freels, Y. Yokoyama, M.L. Benson, B.A. Green, T.A. Saleh, R.L. McDaniels, R.V. Steward, R.A. Buchanan, C.T. Liu, C.R. Brooks, Intermetallics 12 (2004) 1219-1227.

[429] G.Y. Wang, P.K. Liaw, A. Peker, B. Yang, M.L. Benson, W. Yuan, W.H. Peter, L. Huang, M. Freels, R.A. Buchanan, C.T. Liu, C.R. Brooks, Intermetallics 13 (2005) 429-435.

[430] G.Y. Wang, P.K. Liaw, A. Peker, M. Freels, W.H. Peter, R.A. Buchanan, C.R. Brooks, Intermetallics 14 (2006) 1091-1097.

[431] G.Y. Wang, P.K. Liaw, Y. Yokoyama, W.H. Peter, B. Yang, M. Freels, R.A. Buchanan, C.T. Liu, C.R. Brooks, J. Alloys Compd. 434-435 (2007) 68-70.

[432] G.Y. Wang, P.K. Liaw, Y. Yokoyama, A. Peker, W.H. Peter, B. Yang, M. Freels, Z.Y. Zhang, V. Keppens, R. Hermann, R.A. Buchanan, C.T. Liu, C.R. Brooks, Intermetallics 15 (2007) 663-667.

[433] Y. Yokoyama, P.K. Liaw, M. Nishijima, K. Hiraga, R.A. Buchanan, A. Inoue, Mater. Trans., JIM 47 (2006) 1286-1293.

[434] D.C. Qiao, G.Y. Wang, P.K. Liaw, V. Ponnambalam, S.J. Poon, G.J. Shiflet, J. Mater. Res. 22 (2007) 544-550.

[435] D. Qiao, G. Fan, P. Liaw, H. Choo, Int. J. Fatigue 29 (2007) 2149-2154.

[436] G.Y. Wang, P.K. Liaw, M.L. Morrison, Intermetallics 17 (2009) 579-590.

[437] G.Y. Wang, P.K. Liaw, JOM 62 (2010) 25-33.

[438] H.L. Jia, F.X. Liu, Z.N. An, W.D. Li, G.Y. Wang, J.P. Chu, J.S.C. Jang, Y.F. Gao, P.K. Liaw, Thin Solid Films 561 (2014) 2-27. 
[439] K.M. Flores, W.L. Johnson, R.H. Dauskardt, Scripta Mater. 49 (2003) 1181-1187.

[440] D.C. Qiao, P.K. Liaw, C. Fan, Y.H. Lin, G.Y. Wang, H. Choo, R.A. Buchanan, Intermetallics 14 (2006) 1043-1050.

[441] M.E. Launey, D.C. Hofmann, W.L. Johnson, R.O. Ritchie, PNAS 106 (2009) 4986-4991.

[442] J.W. Qiao, E.W. Huang, G.Y. Wang, H.J. Yang, W. Liang, Y. Zhang, P.K. Liaw, Mater. Sci. Eng. A 563 (2013) 101-105.

[443] J.W. Qiao, S.G. Ma, G.Y. Wang, F. Jiang, P.K. Liaw, Y. Zhang, Metall. Mater. Trans. A 42 (2011) 2530-2534.

[444] Y. Wu, Y. Xiao, G. Chen, C.T. Liu, Z. Lu, Adv. Mater. 22 (2010) 2770-2773.

[445] P. Paris, F. Erdogan, J. Basic Eng. 85 (1963) 528-534.

[446] H. Nisitani, D.H. Chen, Trans. Jpn. Soc. Mech. Eng. 50 (1984) 1077-1082.

[447] G.Y. Wang, P.K. Liaw, X.Q. Jin, Y. Yokoyama, E.W. Huang, F. Jiang, L.M. Keer, A. Inoue, J. Appl. Phys. 108 (2010) 113512.

[448] C.C. Wang, Y.W. Mao, Z.W. Shan, M. Dao, J. Li, J. Sun, E. Ma, S. Suresh, PNAS 110 (2013) 19725-19730.

[449] W.H. Jiang, Atzmon M, Acta Mater. 51 (2003) 4095-4105.

[450] D. Turnbull, J. Appl. Phys. 21 (1950) 1022-1028.

[451] P.G. Debenedetti, Metastable Liquids: Concepts and Principles in: Princeton Univ Press, Princeton, 1996.

[452] T. Kawasaki, H. Tanaka, PNAS 107 (2010) 14036-14041.

[453] T. Gloriant, J. Non-Cryst. Solids 316 (2003) 96-103.

[454] Z. Parlar, M. Bakkal, A.J. Shih, Intermetallics 16 (2008) 34-41.

[455] H.W. Jin, R. Ayer, J.Y. Koo, R. Raghavan, U. Ramamurty, J. Mater. Res. 22 (2007) 264-273.

[456] C. Tam, C. Shek, Mater. Sci. Eng. A 384 (2004) 138-142.

[457] X. Fu, T. Kasai, M.L. Falk, D.A. Rigney, Wear 250 (2001) 409-419.

[458] X. Fu, J. Non-Cryst. Solids 317 (2003) 206-214.

[459] A.W.J. De Gee, Wear 92 (1983) 317-317.

[460] H.G. Feller, Wear 92 (1983) 317-318.

[461] R. Klinger, H.G. Feller, Wear 86 (1983) 287-297.

[462] S. Li, Y.Wang, Wear 147 (1991) 275-284.

[463] C.J. Wong, J.C.M. Li, Wear 98 (1984) 45-61.

[464] I.M. Hutchings, Tribology: friction and wear of engineering materials, Edward Arnold, London, 1992.

[465] A.L. Greer, K.L. Rutherford, I.M. Hutchings, Int. Mater. Rev. 47 (2002) 87-112.

[466] X. Fu, D.A. Rigney, Mater. Res. Soc. Symp. Proc. 554 (1998) 437-442.

[467] K. Ozawa, H. Wakasugi, K. Tanaka, IEEE Trans. Magn. 20 (1984) 425-430.

[468] P.J. Blau, Wear 250 (2001) 431-434.

[469] M. Bakkal, Intermetallics 18 (2010) 1251-1253.

[470] M. Ishida, H. Takeda, N. Nishiyama, K. Kita, Y. Shimizu, Y. Saotome, A. Inoue, Mater. Sci. Eng. A 449-451 (2007) 149-154. 
[471] E. Fleury, S. Lee, H. Ahn, W. Kim, D. Kim, Mater. Sci. Eng. A 375-377 (2004) 276-279.

[472] K. Miyoshi, D.H. Buckley, Wear 110 (1986) 295-313.

[473] H. Wu, I. Baker, Y. Liu, X. Wu, P.R. Munroe, Intermetallics 25 (2012) $115-125$

[474] H. Wu, I. Baker, Y. Liu, X.L. Wu, Trans. Nonferrous Met. Soc. China 22 (2012) 585-589.

[475] H. Wu, I. Baker, Y. Liu, X.L. Wu, P.R. Munroe, J.G. Zhang, Intermetallics 35 (2013) 25-32.

[476] J.F. Archard, J. Appl. Phys. 24 (1953).

[477] M. Siegrist, E. Amstad, J. Loffler, Intermetallics 15 (2007) 1228-1236.

[478] J. Wang, H. Danninger, Wear 222 (1998) 49-56.

[479] H.J. Yang, Y. Liu, T. Zhang, H.P. Wang, B. Tang, J.W. Qiao, J. Mater. Sci. Technol. 30 (2014) 576-583.

[480] M.L. Rahaman, L. Zhang, Wear 320 (2014) 77-86.

[481] S.J. Pang, T. Zhang, K. Asami, A. Inoue, Acta Mater. 50 (2002) 489-497.

[482] S.F. Guo, K.C. Chan, S.H. Xie, P. Yu, Y.J. Huang, H.J. Zhang, J. Non-Cryst. Solids 369 (2013) 29-33.

[483] Z.M. Wang, Y.T. Ma, J. Zhang, W.L. Hou, X.C. Chang, J.Q. Wang, Electrochim. Acta 54 (2008) 261-269.

[484] B.A. Green, R.V. Steward, I. Kim, C.K. Choi, P.K. Liaw, K.D. Kihm, Y. Yokoyama, Intermetallics 17 (2009) 568-571.

[485] Y.H. Li, W. Zhang, F.X. Qin, A. Makino, J. Alloys Compds. 615 (2014) S71-S74.

[486] W.H. Jiang, F. Jiang, B.A. Green, F.X. Liu, P.K. Liaw, H. Choo, K.Q. Qiu, Appl. Phys. Lett. 91 (2007) 041904.

[487] X.H. Chen, B.Y. Zhang, G.L. Chen, X.D. Hui, X.R. Xing, Intermetallics 19 (2011) 1913-1918.

[488] Y. Gu, Z. Zheng, S. Niu, W. Ge, Y. Wang, J. Non-Cryst. Solids 380 (2013) $135-140$

[489] M.R. Debnath, D.-H. Kim, E. Fleury, Intermetallics 22 (2012) 255-259.

[490] F. Yang, H.F. Tian, A.D. Lan, H.F. Zhou, B.C. Wang, H.J. Yang, J.W. Qiao, Metall. Mater. Trans. A 46 ( 2014) 2399-2403.

[491] F. Yang, A.D. Lan, H.F. Zhou, H.J. Yang, B.C. Wang, Y.S. Wang, J.W. Qiao, Submitted.

[492] H.F. Tian, A.D. Lan, Y.S. Wang, H.J. Yang, S.P. Pan, J.W. Qiao, Mater. Chem. Phys. 162 (2015) 326-331.

[493] L. Wang, Y.S. Chao, Mater. Lett. 69 (2012) 76-78.

[494] D.P. Wang, S.L. Wang, J.Q. Wang, Corros. Sci. 59 (2012) 88-95.

[495] A. Gebert, U. Kuehn, S. Baunack, N. Mattern, L. Schultz, Mater. Sci. Eng. A 415 (2006) 242-249.

[496] C.L. Qin, W. Zhang, K. Asami, H. Kimura, X.M. Wang, A. Inoue, Acta Mater. 54 (2006) 3713-3719.

[497] J. Wang, S. Huang, Y. Wei, S. Guo, F. Pan, Mater. Lett. 91 (2013) 311-314. 
[498] B. Zberg, P.J. Uggowitzer, J.F. Loffler, Nat. Mater. 8 (2009) 887-891.

[499] J.D. Cao, N.T. Kirkland, K.J. Laws, N. Birbilis, M. Ferry, Acta Biomater. 8 (2012) 2375-2383.

[500] H.F. Li, X.H. Xie, K. Zhao, Y.B. Wang, Y.F. Zheng, W.H. Wang, L. Qin, Acta Biomater. 9 (2013) 8561-8573.

[501] M. Nastasi, F.W. Saris, L.S. Hung, J.W. Mayer, J. Alloys Compd. 58 (1985) 3052-3058.

[502] J. Rivory, J.M. Frigerio, M. Harmelin, A. Quivy, Y. Calvayrac, J. Bigot, Thin Solid Films 89 (1982) 323-327.

[503] Q.M. Chen, Y.D. Fan, H.D. Li, Mater. Lett. 6 (1988) 311-315.

[504] R.B. Schwarz, W.L. Johnson, Phys. Rev. Lett. 51 (1983) 415.

[505] R.J. Highmore, J.E. Evetts, A.L. Greer, R.E. Somekh, Appl. Phys. Lett. 50 (1987) 566.

[506] B.X. Liu, W.S. Lai, Z.J. Zhang, Adv. Phys. 50 (2001) 367-429.

[507] A. Inoue, Acta Mater. 48 (2000) 279-306.

[508] C.J. Chen, J.C. Huang, H.S. Chou, Y.H. Lai, L.W. Chang, X.H. Du, J.P. Chu, T.G. Nieh, J. Alloys Compd. 483 (2009) 337-340.

[509] H.S. Chou, J.C. Huang, L.W. Chang, Surf. Coat.Technol. 205 (2010) 587-590.

[510] M. Stoudt, R.C. Cammarata, R.E. Ricker, Scripta Mater. 43 (2000) 491-496.

[511] K.R. Kim, C.M. Suh, R.I. Murakami, C.W. Chung, Surf. Coat. Technol. 171 (2003) 15-23.

[512] J.M. Cairney, R. Tsukano, M.J. Hoffman, M. Yang, Acta Mater. 52 (2004) 3229-3237.

[513] J.P. Chu, J.E. Greene, J.S.C. Jang, J.C. Huang, Y.L. Shen, P.K. Liaw, Y. Yokoyama, A. Inoue, T.G. Nieh, Acta Mater. 60 (2012) 3226-3238.

[514] J.P. Chu, J.C. Huang, J.S.C. Jang, Y.C. Wang, P.K. Liaw, JOM 62 (2010) 19-24.

[515] J.P. Chu, J.S.C. Jang, J.C. Huang, H.S. Chou, Y. Yang, J.C. Ye, Y.C. Wang, J.W. Lee, F.X. Liu, P.K. Liaw, Y.C. Chen, C.M. Lee, C.L. Li, C. Rullyani, Thin Solid Films 520 (2012) 5097-5122.

[516] Y.Z. Chang, P.H. Tsai, J.B. Li, H.C. Lin, S.C.J. Jang, C. Li, G.J. Chen, Y.C. Chen, J.P. Chu, P.K. Liaw, Thin Solid Films 544 (2013) 331 - 334.

[517] G. Jaeger, B. Endler, K. Bartsch, M. Heilmaier, A. Leonhardt, Surf. Coat. Technol. 150 (2002) 282-289.

[518] J. Mackerle, Modell. Simul. Mater. Sci. Eng. 13 (2005) 935.

[519] R. Montanari, A. Sili, G. Costanza, Compos. Sci. Technol. 61 (2001) 2047-2054.

[520] F.X. Liu, C.L. Chiang, J.P. Chu, Y.F. Gao, P.K. Liaw, Mater. Res. Soc. Symp. Proc. 903E (2006) 0903-Z0914-0913.0901.

[521] F.X. Liu, P.K. Liaw, W.H. Jiang, C.L. Chiang, Y.F. Gao, Y.F. Guan, J.P. Chu, P.D. Rack, Mater. Sci. Eng. A 468-470 (2007) 246-252.

[522] J.P. Chu, C.M. Lee, R.T. Huang, P.K. Liaw, Surf. Coat. Technol. 205 (2011) 4030-4034. 
[523] http://www.aksteel.com/pdf/markets_products/stainless/austenitic/316_316L Data_Bulletin.pdf.

[524] J. Chu, C. Liu, T. Mahalingam, S. Wang, M. O'Keefe, B. Johnson, C. Kuo, Phys. Rev. B 69 (2004) 113410.

[525] J.P. Chu, C.T. Lo, Y.K. Fang, B.S. Han, Appl. Phys. Lett. 88 (2006) 012510.

[526] J.P. Chu, JOM 61 (2009) 72-75.

[527] J.P. Chu, C.Y. Wang, L.J. Chen, Q. Chen, Surf. Coat. Technol. 205 (2011) 2914-2918.

[528] C.S. Chen, P. Yiu, C.L. Li, J.P. Chu, C.H. Shek, C.H. Hsueh, Mater. Sci. Eng. A 608 (2014) 258-264.

[529] L. Cheng, Z.M. Jiao, S.G. Ma, J.W. Qiao, Z.H. Wang, J. Appl. Phys. 115 (2014) 084907.

[530] C.Y. Chuang, J.W. Lee, C.L. Li, J.P. Chu, Surf. Coat. Technol. 215 (2013) 312-321.

[531] H.S. Chou, J.C. Huang, L.W. Chang, T.G. Nieh, Appl. Phys. Lett. 93 (2008) 191901.

[532] H.Q. Li, L. Li, C. Fan, H. Choo, P.K. Liaw, J. Mater. Res. 22 (2007) 508-513.

[533] J.W. Rudnicki, J.R. Rice, J. Mech. Phys. Solids 23 (1975) 371-394.

[534] Y.F. Gao, L. Wang, H. Bei, T.G. Nieh, Acta Mater. 59 (2011) 4159-4167.

[535] Y.F. Gao, Modell. Simul. Mater. Sci. Eng. 14 (2006) 1329-1345.

[536] Y.C. Wang, C.Y. Wu, J.P. Chu, P.K. Liaw, Metall. Mater. Trans. A 41 (2010) 3010-3017.

[537] A.R. Leach, Molecular Modeling: Principles and Applications, 2nd ed., Prentice Hall, Upper Saddle River, NJ, 2001.

[538] H.C. Lin, J.G. Chang, S.P. Ju, C.C. Hwang, Proc. R. Soc. A Math. Phys. Eng. Sci. 46 (2005) 3977-3998.

[539] L.L. Zheng, Y.F. Gao, S.Y. Lee, R.I. Barabash, J.H. Lee, P.K. Liaw, J. Mech. Phys. Solids 59 (2011) 2307-2322.

[540] S. Xie, E.P. George, Acta Mater. 56 (2008) 5202-5213.

[541] Z.N. An, W.D. Li, F.X. Liu, P.K. Liaw, Y.F. Gao, Metall. Mater. Trans. A 43 (2012) 2729-2741.

[542] L.W. Ren, M.M. Meng, Z. Wang, F.Q. Yang, H.J. Yang, T. Zhang, J.W. Qiao, Intermetallics 57 (2015) 121-126.

[543] M.M. Meng, Z.P. Gao, L.W. Ren, H.J. Yang, S.G. Ma, Z.H. Wang, J.W. Qiao, Mater. Sci. Eng. A 615 (2014) 240-246.

[544] J.W. Qiao, Y. Zhang, P.K. Liaw, G.L. Chen, Scripta Mater. 61 (2009) 1087-1090.

[545] Y. Shao, K.F. Yao, M. Li, X. Liu, Appl. Phys. Lett. 103 (2013) 171901.

[546] B.B. Sun, M.L. Sui, Y.M. Wang, G. He, J. Eckert, E. Ma, Acta Mater. 54 (2006) 1349-1357.

[547] Y.L. Hao, S.J. Li, B.B. Sun, M.L. Sui, R. Yang, Phys. Rev. Lett. 98 (2007) 216405.

[548] Y.S. Wang, G.J. Hao, Y. Zhang, J.P. Lin, L. Song, J.W. Qiao, Surf. Interface Anal. 46 (2014) 293-296. 
[549] T. Zhang, H.Y. Ye, J.Y. Shi, H.J. Yang, J.W. Qiao, J. Alloy Compds. 583 (2014) 593-597.

[550] R.T. Ott, F. Sansoz, J.F. Molinari, J. Almer, K.T. Ramesh, T.C. Hufnagel, Acta Mater. 53 (2005) 1883-1893.

[551] T. Zhang, J.Y. Shi, J.W. Qiao, H.J. Yang, P.K. Liaw, Metall. Mater. Trans. A 45 (2014) 2382-2388.

[552] B. Jakobsen, H.F. Poulsen, U. Lienert, J. Almer, S.D. Shastri, H.O. Sørensen, Science 312 (2006) 889-892.

[553] Y. Wang, Z. Guo, R. Ma, G. Hao, Y. Zhang, J. Lin, M. Sui, Prog. Mater. Sci. 24 (2014) 121-127.

[554] X.J. Liu, Y. Xu, X. Hui, Z.P. Lu, F. Li, G.L. Chen, J. Lu, C.T. Liu, Phys. Rev. Lett. 105 (2010) 155501.

[555] H.L. Peng, M. Li, W.H. Wang, Phys. Rev. Lett. 106 (2011) 135503.

[556] Q.K. Li, M. Li, Appl. Phys. Lett. 91 (2007) 231905.

[557] Y.Q. Cheng, E. Ma, Acta Mater. 59 (2011) 1800-1807.

[558] H.Y. Zhang, G.P. Zheng, J. Alloy Compds. 586 (2014) S262-S266.

[559] Y. Shen, G.P. Zheng, Scripta Mater. 63 (2010) 181-184.

[560] F. Abdeljawad, M. Haataja, Phys. Rev. Lett. 105 (2010) 125503.

[561] Y. Jiang, K. Qiu, Mater. Des. 65 (2015) 410-416.

[562] C. Jeon, H. Lee, C.P. Kim, S.-H. Joo, H.S. Kim, S. Lee, Metall. Mater. Trans. A 46 (2015) 235-250.

[563] J. Booth, J. Lewandowski, J. Carter, Microsc. Microanal. 20 (2014) 852-853.

[564] G. Kumar, A. Desai, J. Schroers, Adv. Mater. 23 (2011) 461-476.

[565] The BMG products are provided by DongGuan Eontec Co., Ltd. in China.

[566] C. Suryanarayana, A. Inoue, bulk metallic glasses, CRC Press (2011).

[567] Y.F. Zheng, X.N. Gu, F. Witte, Mater. Sci. Eng. R 77 (2014) 1-34.

[568] Q. Chen, G.A. Thouas, Mater. Sci. Eng. R 87 (2015) 1-57.

[569] M. Krautz, A. Funk, K.P. Skokov, T. Gottschall, J. Eckert, O. Gutfleisch, A. Waske, Scripta Mater. 95 (2015) 50-53.

[570] http://www.techbriefs.com/component/content/article/7-ntb/tech-briefs/manu fac turing-and-prototyping/17444.

[571] J.-Z. Jiang, D. Hofmann, D.J. Jarvis, H.-J. Fecht, Adv. Eng. Mater. 17 (2015) 761-780.

[572] http://www.biotinet.eu/downloads/2nd\%20Workshop\%20Belgium/Stoica-Bi oTi Net2.pdf.

[573] K.H. Lo, C.H. Shek, J.K.L. Lai, Mater. Sci. Eng. R 65 (2009) 39-104.

[574] Q.P. Cao, J.W. Liu, K.J. Yang, F. Xu, Z.Q. Yao, A. Minkow, H.J. Fecht, J. Ivanisenko, L.Y. Chen, X.D. Wang, S.X. Qu, J.Z. Jiang, Acta Mater. 58 (2010) 1276-1292.

[575] K.R. Rim, J.M. Park, W.T. Kim, D.H. Kim, J. Alloy Compds. 579 (2013) 253-258.

[576] S. Sankaranarayanan, V.H. Shankar, S. Jayalakshmi, N.Q. Bau, M. Gupta, J. Alloy Compds. 627 (2015) 192-199. 
[577] J. Gao, J. Sharp, D. Guan, W.M. Rainforth, I. Todd, Acta Mater. 86 (2015) 208-215.

[578] W.H. Wang, Adv. Mater. 21 (2009) 4524-4544. 


\section{Table Captions}

Table 1: Summaries of Some BMGs with Critical Size $\geq 10 \mathrm{~mm}$

Table 2 Chemical compositions of the composites of E, F, G, and $\mathrm{H}$ by estimations and measurements [87].

Table 3 Summary of the compressive mechanical properties of the composites at ambient temperature [88].

Table 4 The thermal properties of the $\mathrm{Zr}_{37.5} \mathrm{Ti}_{32.2} \mathrm{Nb}_{7.2} \mathrm{Cu}_{6.1} \mathrm{Be}_{17.0}$ MGMCs with different withdrawal velocities $[69,95]$. 


\section{Figure Captions}

Figure 1 The amorphous structure (a) and the cubic crystalline (b) CuZr alloy by computer simulations.

Figure 2 Different BMG systems with the maximum diameters as well as their discovered years, including Nd- [20], Y- [17], Pt- [33], Cu- [15], Ca- [30], Fe- [25], Co[26], La- [34], Mg- [22], Ni- [32], Ti- [29], and Pd-based BMGs [12].

Figure 3 An optical micrograph showing uniformly-distributed WC particles in the V106 matrix (a) [37], X-ray diffraction patterns of the V106 matrix, the alloy reinforced with 10 vol. \% WC, and pure WC particle (b) [38], and quasi-static compression stress-strain curves of V106 composites reinforced with Ta, W, and WC particles [39].

Figure 4 Scanning-electron micrographic image of the transverse cross section of the cast composite alloy containing $40 \mathrm{vol} \%$ Ti powders [48].

Figure 5 (a) Hardness values of the matrix reinforced by different volumes of the $\mathrm{ZrC}$ particles and (b) linear fitness of the modulus, E, and yielding strength, $\sigma_{y}$, with increasing the volume fraction $\left(\mathrm{V}_{\mathrm{f}}\right)$ of $\mathrm{ZrC}$ [42].

Figure 6 SEM micrograph in the backscatter mode of 80 vol. $\% \mathrm{~W}$ wire / bulk metallic glass matrix composite. The sample is polished and cut normal to the uniaxial reinforcement [56].

Figure 7 SEM backscattered-electron image of in-situ composite microstructure (Inset: X-ray diffraction pattern for the $\left(\mathrm{Zr}_{75} \mathrm{Ti}_{18.34} \mathrm{Nb}_{6.66}\right)_{75} \mathrm{X}_{25}$ in-situ composite [86]. 
Figure 8 Processing map (a quasi-equilibrium pseudo-binary phase diagram) for in-situ $\beta$-phase-reinforced composites during a cooling experiment. The glass-transition temperature, $T_{\mathrm{g}}$, of the matrix is close to that of Vit $1\left(350^{\circ} \mathrm{C}\right)$. The composites with compositions marked E, F, G, and $\mathrm{H}$ were processed and studied to determine their phase fractions to confirm the phase diagram [87].

Figure 9 Backscattered-SEM images of the composites (E, F, G, and $\mathrm{H})$ marked in Figure 8 . The light regions designate the $\beta$ phase, and the dark areas are the amorphous matrix. The insets on the upper left corner show the intensity distributions used in estimating the fractions of each phase [87].

Figure 10 A pseudo ternary phase diagram with apexes of zirconium, (titanium + niobium), and $\mathrm{X}$, where $\mathrm{X}$ represents the moiety $\mathrm{Cu}_{5} \mathrm{Ni}_{4} \mathrm{Be}_{9}[88]$.

Figure 11 X-ray diffraction patterns of four samples with different compositions (labeled as Zr54, Zr56, Zr58, and Zr60, respectively) [88].

Figure 12 SEM images of the cross sections of the composites with the compositions of: (a) Zr54, (b) Zr56, (c) Zr58, and (d) Zr60 [88].

Figure 13 (a) the compressive engineering stress-strain curves of the four composites [88], and (b) the tensile engineering stress-strain curves of the Zr60 composite [90]. Figure 14 Backscattered SEM of the in-situ composite with a composition of $\left(\mathrm{Zr}_{70} \mathrm{Ni}_{10} \mathrm{Cu}_{20}\right)_{82} \mathrm{Ta}_{8} \mathrm{Al}_{10}[113]$.

Figure 15 The crystalline structures of the $\mathrm{B} 2 \mathrm{CuZr}, \mathrm{P} 2_{1} / \mathrm{m}$, and $\mathrm{Cm}$ phases $[136,137]$. Figure 16 Schematic CCT diagram during the quenching process illustrating the formation of CuZr-based BMG composites [137]. 
Figure 17 Dependence of tensile ductility on crystalline volume fraction [132].

Figure 18 Typical schematic illustration of copper-mold suction casting, and the arc melter at The University of Tennessee shown in the inset [141].

Figure 19 Schematic diagram of the apparatus for casting bulk MGMCs [37].

Figure 20 (a) diagram of a water-cooled copper boat used for semisolid processing of in-situ metallic glass matrix composites, and (b) example of a custom coil on a water-cooled copper boat [165].

Figure 21 Plot of shear modulus versus volume fraction of dendrites for the alloy, DH1, its glass matrix and its dendrite (a) [94] and contrast adjusted SEM of the dendrite structure in a bulk metallic glass matrix composite [165].

Figure 22 The Bridgman solidification apparatus used by Qiao et al. (a), and the corresponding illustration exhibited in (b).

Figure 23 XRD patterns (a) and DSC traces (b) of the composites synthesized with different withdrawal velocities [69].

Figure 24 The microstructure of the sample with $\mathrm{v}=1.0 \mathrm{~mm} / \mathrm{s}$ is illustrated in (a) [95], the dependences of spanning lengths of individual dendrite trees on varied withdrawal velocities shown in (b) [95], and the dependences of fracture strengths and plastic strains of the composites developed by the Bridgman solidification on the $\mathrm{v}$ (c), the inset in (c) indicating the sample with $\mathrm{v}=1.0 \mathrm{~mm} / \mathrm{s}$ after bending.

Figure 25 Classification of MGMCs.

Figure 26 A schematic of the free-volume model in metallic glasses. 
Figure 27 (a) AFM image of the nanoscale-periodic corrugation in a Vitreloy 1 metallic glass [210]. (b) Periodic corrugation forms by the occurrence of the local quasi-cleavage due to TTZs near the tip; coalescence of the local quasi-cleavage zone with the crack yields a longer crack [209].

Figure 28 Schematic diagram of the ductile-to-brittle transition mechanism of metallic glasses during fracture. (a) If the curvature radius of the crack tip is greater than the critical wavelength of the meniscus instability, a ductile fracture will occur in the fracture-process zone, where the atomic-cluster motion via shear transformation zones (STZs), as shown in the inset; and (b) in the opposite case, the quasi-brittle fracture through tension transformation zones (TTZs) [see the inset] is possible ahead to the sharp crack tip [217].

Figure 29 Correlations between the evolution of liquid-like zones, deformation map, relaxation spectrum and energy landscape are established during the glass-to-liquid transition [235].

Figure 30 Schematic diagrams of the stick-slip model for a single sample where only a dominant shear band formed (a) and several samples tested simultaneously (b). Figure 31 The relationship among the fracture strength, $T_{\mathrm{g}}$, and molar volume for a variety of BMGs [269].

Figure 32 The SEM images of the S2 composite shown in (a), and the quasi-static compressive stress-strain curves of LM1, S1, and S2 (b) [66].

Figure 33 Illustration of the competitive process between the shear and splitting fracture for the tungsten composite at room temperature: (a) variations of shear and splitting fracture strengths with fiber volume fractions, (b) critical compressive fracture 
lines for shear and splitting fracture modes of the composites with different fiber volume fractions and the stress distribution on the two Mohr circles [296].

Figure 34 Specific strength and plasticity of a series of monolithic BMGs and ex-situ particle-reinforced MGMCs [48].

Figure 35 The compressive engineering stress-strain curves of the $\mathrm{Zr}_{58.5} \mathrm{Ti}_{14.3} \mathrm{Nb}_{5.2} \mathrm{Cu}_{6.1} \mathrm{Ni}_{4.9} \mathrm{Be}_{11.0}$ composite, and its corresponding dendrite and glass matrix.

Figure 36 The compressive stress-strain curves at four different test temperatures (77, 294, 373, and $473 \mathrm{~K})$ [59].

Figure 37 Comparison of the stress-strain responses at various testing temperatures: (a) at room temperature $(300 \mathrm{~K}),(\mathrm{b})$ at high temperature but below $T_{\mathrm{g}}(550 \mathrm{~K}),(\mathrm{c})$ at $T_{\mathrm{g}}$ $(600 \mathrm{~K})$, and $(\mathrm{d})$ above $T_{\mathrm{g}}(650 \mathrm{~K})$. Note that the plots obtained at the strain rate of $10^{-2}$ $\mathrm{s}^{-1}$ were not shown for the sake of clarity [302].

Figure 38 The true stress-strain curves measured at 77 and $298 \mathrm{~K}$ (a) as well as in the supercooled liquid region, $693 \mathrm{~K}$ for 70 -vol. \%-W-containing-Vit106 composites with the SEM image and (b) for 5-vol. \%-Ta-containing Zr-based composites with the backscattering SEM image [307].

Figure 39 Integrated tensile properties of strength and ductility for in-situ MGMCs. Figure 40 (a) the tensile true stress-strain curves of ex-situ porous tungsten / Zr-based metallic-glass-interpenetrating composites, the inset in (a) showing the microstructure [317]; (b) the stress-displacement curves of in-situ dendrite / Ti-based MGMCs at 613 $\mathrm{K}$ with a volume fraction of dendrites of $43 \%$, the inset indicating pictures of the 
microstructure and deformed samples [318]; and (c) the tensile true tress-strain curves at $693 \mathrm{~K}$ for the in-situ Ta-particle-reinforced MGMCs with a composition of $\mathrm{Zr}_{55.9} \mathrm{Cu}_{18.6} \mathrm{Ni}_{10} \mathrm{Al}_{7.5} \mathrm{Ta}_{8}$, the inset giving the picture of the super elongation and microstructures [319].

Figure 41 The tensile stress-strain curve of in-situ dendrite MGMCs at $77 \mathrm{~K}$ (a); the pictures of samples before and after deformation (b); and the diffraction pattern after deformation (c) [322].

Figure 42 (a) schematic of the composite structure consisting of second-phase nanocrystals. Colors are assigned to identify structural features: blue for the glass matrix, grey for the secondary crystalline phase, and green for the glass-crystal interfaces and the free surfaces. (b) three composites embedded with different shapes of nanocrystals, i.e., $b / a=6.5,5.7$, and 3.7. The corresponding volume fractions of the crystalline phase are $16.7 \%, 14.5 \%$, and $9.4 \%$, respectively [327]. (c) atomistic configurations of the structural evolution in 'Composite II' upon deformation. (d) and (e) typical deformation patterns illustrating structural evolution in the composites and monolithic BMG, respectively. (f) atomistic configuration of 'Composite Inclined' at a strain of 15\%. (g) and (h) atomic configurations of 'Composite Random' at various applied strains. (i) atomic configurations of 'Composite Dendritic' upon deformation [327].

Figure 43 Shear-strain contours of a nominal strain of $11.5 \%$ for (a) Comp1, (b)

Comp2, and (c) Comp3, with circular, large dendrites, and small dendrite crystalline 
inclusions, respectively. Dark domains represent a glassy phase, while light domains indicate for ductile phases [326].

Figure 44 (a) typical stress-strain curves for composites with various volume fractions of second-phase nanocrystals. (b) - (d) Atomic configurations at various applied strains demonstrating the structural evolution in 'Composite VII'. The arrow indicates the activation of lattice sliding at a strain of $13 \%$. (e) and (f) schematics illustrating the transition in the governing deformation mechanism of the glass subdomains as the volume fraction of the crystalline second phase increases [327].

Figure 45 Different deformation modes for shear banding and crack propagation in MGMCs: (a) and (b) shear-banding-induced cracking inside the reinforcement after the shear band interacts with reinforcements. $\mathrm{g}=0, \mathrm{w}=0 ; K_{\mathrm{I}}=24.2 \mathrm{MPa} \sqrt{\mathrm{m}}(\mathrm{a})$, and 27.5 MPa $\sqrt{\mathrm{m}}(\mathrm{b})$; (c) propagation of shear bands along the reinforcement surface with a compressive residual stress $\mathrm{g}=-435 \mathrm{MPa} ; \mathrm{w}=0$; (d) shear-band-induced cracking inside the reinforcement with a tensile residual stress, $g=175 \mathrm{MPa}$ at its surface; $\mathrm{w}=$ 0 ; (e) branching of shear bands along the reinforcement surface; $w=0.8 ; g=0$. (f) shear banding at opposite sides of the reinforcement surface; $\mathrm{w}=1 ; \mathrm{g}=0 . \mathrm{K}_{\mathrm{I}}=27.5$ $\mathrm{MPa} \sqrt{\mathrm{m}}$ in (c)-(f) [325].

Figure 46 Low-magnification optical photographs of the dynamically deformed compressive specimen for the (a) stainless steel (S) and (b) Tantalum (T) fiber reinforced specimens [65].

Figure 47 The engineering stress-strain curve of the present in-situ metallic glass matrix composites upon quasi-static compression (a); the fractograph of the deformed 
samples upon quasi-static loading (b); the magnified deformation region near the crack (c); the engineering stress-strain curves upon high-speed dynamic loading (d); and the SEM image of the magnified lateral surface (e) [348].

Figure 48 The stress-strain curves of $\mathrm{Cu}_{46} \mathrm{Zr}_{46} \mathrm{Al}_{8}$ MGMCs upon dynamic loading [322].

Figure 49 The stress-strain curve of monolithic BMGs upon dynamic tension shown in (a) [322]; and typical true stress strain curves of ex-situ porous W / MGMCs under tension at different strain rates shown in (b) [352]; the inset in (a) and (b) showing the lateral surface and fracture morphology, respectively.

Figure 50 Micrograph of a deformed notch in a glassy $\mathrm{Pd}_{79} \mathrm{Ag}_{3.5} \mathrm{P}_{6} \mathrm{Si}_{9.5} \mathrm{Ge}_{2}$ specimen showing extensive plastic shielding of an initially sharp crack [371].

Figure 51 Ranges of fracture toughness versus strength shown for different engineering materials [404].

Figure 52 R-curves showing resistance to fracture in terms of the stress intensity, $K_{\mathrm{J}}$ as a function of crack extension, the DH1 and DH3 composite BMG, monolithic Vitreloy $1 \mathrm{BMG}$, and a 17-7 PH stainless steel [412].

Figure 53 The stress range vs. cycles to failure for three-point-bending (a) and four-point-bending (b) fatigue of BMGs [437].

Figure 54 The $\mathrm{S}-\mathrm{N}$ curves of $\mathrm{Zr}_{58.5} \mathrm{Ti}_{14.3} \mathrm{Nb}_{5.2} \mathrm{Cu}_{6.1} \mathrm{Ni}_{4.9} \mathrm{Be}_{11.0}$ MGMCs upon four-point-bending and tension-tension fatigue (a) [442], and the S-N curves of $\mathrm{Zr}_{48} \mathrm{Cu}_{47.5} \mathrm{Co}_{0.5} \mathrm{Al}_{4}$ composites upon compression-compression fatigue (b) (unpublished data). 
Figure 55 (a) SEM of the lateral surface near the fracture surface of Zr-based MGMCs with a stress range of $792 \mathrm{MPa}$ and cycle number to failure of 14,776; (b) SEM of a fatigue initiation region; (c) SEM of the crack-growth region; (d) SEM of magnified crack-propagation region; (e) SEM of crack-growth region and fast fracture region discerned by a dash line; and (f) SEM of the magnified fast fracture region [442]. Figure 56 (a) Fatigue fractography of the composites subjected to a stress range of 810 MPa. (b) The enlarged part in stage II near the arresting line in (a) [443].

Figure 57 (a) Fatigue-crack-growth rates $(\mathrm{da} / \mathrm{dN})$ vs. the stress-intensity-factor range $(\Delta \mathrm{K})$ for Zr-based BMGs and BMG composites and some crystalline alloys (CT: compact tension, SE: single-edge notch, C: composite, CCT: center-cracked tension, NC: nano-crystalline phase) [436] and (b) The S-N curves of Zr-based BMGs, as well as some typical crystalline alloys [436].

Figure $58 \mathrm{MD}$ simulations on fatigue tests of Al-Fe metallic glass. Two identical specimens were tested under cyclic straining and monotonic loading. (A) The crystalline phase can be seen clearly after 275 cycles. (B) The sample remained amorphous under monotonic loading even though the strain reached 8\%. (C) The distribution of the atom fraction vs. the square of nanoaffine displacement $\left(D^{2}\right)$ for cycle nos. 1, 20, 70, 175, and 275. (D) Both the averaged $\mathrm{D}^{2}$ (solid black line) and the average grain size (curve with circles) increased with increasing cycle number [448]. Figure 59 (a) The friction coefficient and (b) wear rate of $\mathrm{Zr}_{41.2} \mathrm{Ti}_{13.8} \mathrm{Cu}_{12.5} \mathrm{Ni}_{10} \mathrm{Be}_{22.5}$ BMG as a function of normal load, and (c) the friction coefficient as a function of sliding speed [457]. 
Figure 60 The wear volume with the sliding distance in four BMGs (a) [465] and the wear rate of the $\mathrm{Zr}_{41.2} \mathrm{Ti}_{13.8} \mathrm{Cu}_{12.5} \mathrm{Ni}_{10} \mathrm{Be}_{22.5} \mathrm{BMG}$ with the sliding distance (b) [466]. Figure 61 (a) Bright-field TEM image of subsurface region in wear pin for test performed in oxygen, and (b) close-up view of the near-surface region in (a) [473]. Figure 62 The friction coefficient as a function of sliding time for in-situ dendrite / Zr-based MGMCs under different normal loads (a) and sliding velocities (b) [479]. Figure 63 Polarization curves of Ti40Zr24V12Cu5Be19 amorphous alloy in different solutions [490].

Figure 64 Schematic illustration of the compositional depth profiles of $\mathrm{Ti}$ and $\mathrm{Zr}$ oxidized states in passive films for $\mathrm{Ti}_{46} \mathrm{Zr}_{20} \mathrm{~V}_{12} \mathrm{Cu}_{5} \mathrm{Be}_{17}$ amorphous alloy after elechemical corrosion experiment in acid solution [491].

Figure 65 Polarization curves of Zr-based MGMCs in different concentration $\mathrm{NaCl}$ solutions (a), and electrochemical impedance spectroscopy (EIS) of Zr-based MGMCs at open circuit potential in different concentration $\mathrm{NaCl}$ solution (b) [492].

Figure 66 A review of the ductility and yield strength of different kinds of coatings, including TFMGs, metallic coatings, and ceramic coatings applied to improve mechanical properties of substrate materials [438] .

Figure 67 (a) S-N curves for all the reported TFMGs on different kinds of substrates. Five regions are separated in (a) by: A) low fatigue-endurance limit region; B) medium fatigue-endurance limit region; C) high fatigue-endurance limit region; D) tension-tension region; and E) monolithic $\mathrm{BMG}$ region [438], and (b) the improvement 
of fatigue-endurance limit vs. UTS for all the reported substrate materials, including the 316L steel, Ni-based alloy, Zr-based alloy, Ti-based alloy, and Al-based alloy [438]. Figure 68 TEM bright-field images and diffraction patterns from Fe-based TFMGs revealing phase evolution during 1 min annealing: amorphous matrix with $\gamma$-fcc FeNi nanocrystallites (as-deposited) $\rightarrow$ growth of nanocrystals (up to $400{ }^{\circ} \mathrm{C}$ ) $\rightarrow$ amorphous $\left(500{ }^{\circ} \mathrm{C}\right.$ and $\left.550{ }^{\circ} \mathrm{C}\right) \rightarrow$ formation of cubic $\mathrm{Fe}(\mathrm{Ni})$ crystals $\left(600^{\circ} \mathrm{C}\right)$ with minor cubic FeNi phase $\left(650^{\circ} \mathrm{C}\right.$ to $\left.700{ }^{\circ} \mathrm{C}\right) \rightarrow$ formation and grain growth of FeNi phase $\left(750{ }^{\circ} \mathrm{C}\right)$ [525].

Figure 69 S-N curves of a bare 316L stainless-steel substrate and coated specimens with as-deposited and annealed 200-nm-thick $\mathrm{Zr}_{53} \mathrm{Cu}_{29} \mathrm{Al}_{12} \mathrm{Ni}_{6}$ TFMGs under four-point-bending fatigue tests [515].

Figure 70 (a) MD Simulated $80 \AA$-thick $\mathrm{Cu}_{47} \mathrm{Zr}_{47} \mathrm{Al}_{6}$ TFMG (Cu atoms = green, $\mathrm{Zr}=$ blue, and $\mathrm{Al}=\mathrm{red}$ ) on a Ti substrate. (b) MD simulation of the engineering strain $\varepsilon 11$ under a rigid indent in a $\mathrm{Cu}_{47} \mathrm{Zr}_{47} \mathrm{Al}_{6}$ TFMG on a rigid Ti substrate [515].

Figure 71 ABAQUS contours of equivalent plastic strains in (a) a bare BMG substrate and (b) an MG-coated BMG specimen after four-point-bending deformation [438]. Figure 72 ABAQUS model of a half-symmetric Rockwell indention in a metallic glass, used for predicting shear-band directions in (b) a BMG substrate, and (c) a Ti-coated BMG with a film/substrate thickness ratio of $1 / 20$, having $\mu+\beta=0$ and $v=0.3$ [438]. Figure 73 TEM bright-field image and the corresponding SAED pattern (inset) for the dendrites shown in (a); low-magnification HRTEM image of dendrites taken away from the interface showing some Moiré patterns (b) and high-magnification TEM 
bright-field image showing a step morphology at the interface; HRTEM image close to the interface showing lattice distortion (c) and FFT pattern of the area marked by the rectangle in the inset, the corresponding IFFT pattern shown in (d); HRTEM image of the glass matrix indicating the presence of nano-crystallites (e), the IFFT pattern (inset) of nano-crystallites giving a periodic lattice contrast [544].

Figure 74 The TEM BF image of the composite with a low magnification shown in a; the HRTEM image of the glass matrix exhibited in $b$ and its corresponding SAED pattern in the inset; the BF and DF images of the deformed dendrites shown in $\mathrm{c}$ and $\mathrm{d}$, respectively; the SAED patterns of the dendrites marked by rectangles in $\mathrm{c}$ and $\mathrm{d}$ shown in the insets of $\mathrm{c}$ and $\mathrm{d}$, respectively; the IFFT image within the shear bands marked by arrows in $\mathrm{c}$ and $\mathrm{d}$ shown in e; the BF and DF images of the dendrites separated by DDWs, denoted by arrows, exhibited in $\mathrm{f}$ and $\mathrm{g}$, respectively; the high-magnified BF image of $\mathrm{CB} B$, as marked in $\mathrm{f}$ and $\mathrm{g}$, shown in the inset of Figure 5g; the IFFT and FFT patterns taken from the DDW near the interface of CBs A and $\mathrm{B}$ displayed in $\mathrm{h}$ and the inset of $\mathrm{h}$, respectively; the BF images of the tensile- and shear-induced separations shown in i and j, respectively; the HRTEM image near the interface of the dendrites and glass matrix exhibited in k; and the IFFT image in 1 of the dendrites marked by a rectangle in $\mathrm{k}[101]$.

Figure 75 The observation of the crack deflection and propagation between the two phases: (a) from the amorphous phase to the dendrite phase, and (b) from the dendrite phase to the amorphous phase [553]. 
Figure 76 Surface plots of shear stress fields in MGMCs containing dendrites with a fracture energy $e_{\mathrm{c}}=145.2 \mathrm{~J} / \mathrm{m}^{2}$. (a) Rotation angle $\theta=0$ deg. The color bars marked as $\mathrm{S}_{\mathrm{m}}$ and $\mathrm{S}_{\mathrm{d}}$ at the left are for the maximum shear stresses in the BMG matrix and dendrites, respectively. (b) Rotation angle $\theta=15 \mathrm{deg}$. The color bars marked as $\mathrm{S}_{\mathrm{m}}$ and $\mathrm{S}_{\mathrm{d}}$ at the right are for the maximum shear stresses in the glass matrix and dendrites, respectively [558].

Figure 77 Different forms in which BMGs have been produced [565].

Figure 78 High-performance knives based on Ti-based MGMCs [570].

Figure 79 Examples of a variety of net-shaped parts of the Ti-based BMG matrix composite DV1 (TiZrVCuBe) showing how complex shapes can be cast with Ti-based composites. Bolts, washers, hollow tubes, and rods are demonstrated [571].

Figure 80 Various processing methods to fabricate MGMCs [572]. 


\begin{tabular}{lllllll}
\hline Table 1: & Summaries of Some BMGs with Critical Size $\geq \mathbf{1 0} \mathbf{~ m m}$ & & \\
\hline System & Alloys & $\begin{array}{l}\text { Critical } \\
\text { Sizes, } \mathbf{D}_{\mathbf{c}} \\
(\mathbf{m m})\end{array}$ & Method & $\begin{array}{l}\text { Yea } \\
\text { Pd-based }\end{array}$ & Ref. \\
& & 10 & Fluxing & 198 & 13 \\
& $\mathrm{Pd}_{40} \mathrm{Ni}_{40} \mathrm{P}_{20}$ & $\mathrm{Pd}_{40} \mathrm{Cu}_{30} \mathrm{Ni}_{10} \mathrm{P}_{20}$ & & & 4 & \\
\hline
\end{tabular}




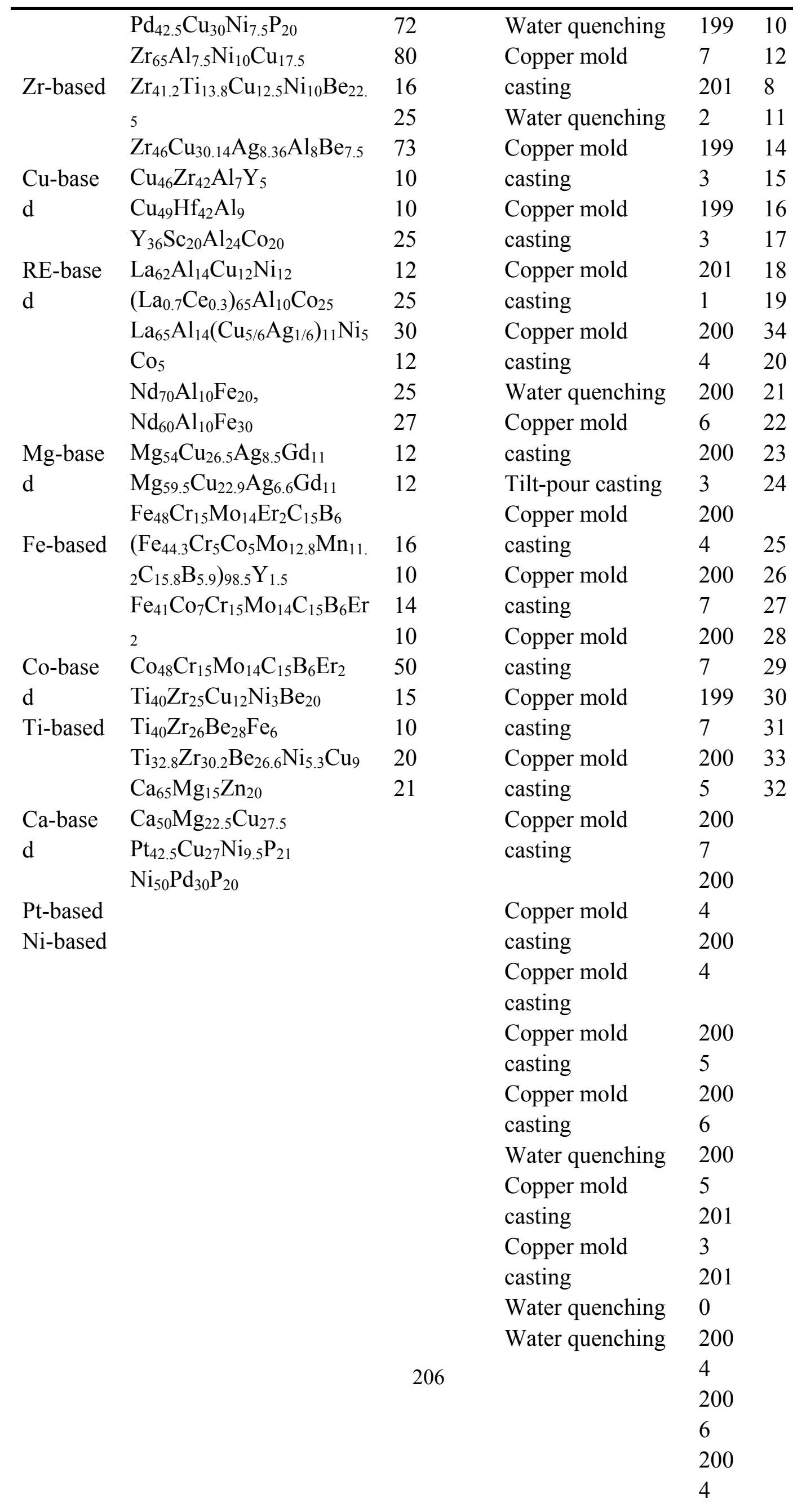


Table 2 Chemical compositions of the composites of E, F, G, and $\mathrm{H}$ by estimations and measurements [87].

\begin{tabular}{llll}
\hline $\begin{array}{l}\text { Composites } \\
\text { label }\end{array}$ & $\begin{array}{l}\text { Chemical compositions } \\
\text { E }\end{array}$ & $\begin{array}{l}\text { Estimated vol. } \\
\% \text { of the } \beta \\
\text { phase }\end{array}$ & $\begin{array}{l}\text { Measured vol. } \\
\% \text { of the } \beta \\
\text { phase }\end{array}$ \\
\hline $\mathrm{F}$ & $\left(\mathrm{Zr}_{75} \mathrm{Ti}_{15} \mathrm{Nb}_{10}\right)_{80}\left[\mathrm{Be}_{50}\left(\mathrm{Cu}_{55} \mathrm{Ni}_{45}\right)_{50}\right]_{20}$ & 55 & $57 \pm 3$ \\
$\mathrm{G}$ & $\left(\mathrm{Zr}_{75} \mathrm{Ti}_{15} \mathrm{Nb}_{10}\right)_{85}\left[\mathrm{Be}_{50}\left(\mathrm{Cu}_{55} \mathrm{Ni}_{45}\right)_{50}\right]_{15}$ & 68 & $67 \pm 3$ \\
$\mathrm{H}$ & $\left(\mathrm{Zr}_{75} \mathrm{Ti}_{15} \mathrm{Nb}_{10}\right)_{90}\left[\mathrm{Be}_{50}\left(\mathrm{Cu}_{55} \mathrm{Ni}_{45}\right)_{50}\right]_{10}$ & 80 & $81 \pm 2$ \\
\hline
\end{tabular}


Table 3 Summary of the compressive mechanical properties of the composites at ambient temperature [88].

\begin{tabular}{cccccc}
\hline Alloys & $\begin{array}{c}\sigma_{\mathrm{y}} \\
(\mathrm{MPa})\end{array}$ & $\varepsilon_{\mathrm{y}}(\%)$ & $\begin{array}{c}\sigma_{\max } \\
(\mathrm{MPa})\end{array}$ & $\varepsilon_{\mathrm{f}}(\%)$ & Ref. \\
\hline $\mathrm{Zr54}\left(\mathrm{Zr}_{54.0} \mathrm{Ti}_{13.2} \mathrm{Nb}_{4.8} \mathrm{Cu}_{7.8} \mathrm{Ni}_{6.2} \mathrm{Be}_{14.0}\right)$ & 1,390 & 1.9 & 1,710 & 6.7 & {$[88]$} \\
$\mathrm{Zr56}\left(\mathrm{Zr}_{56.2} \mathrm{Ti}_{13.8} \mathrm{Nb}_{5.0} \mathrm{Cu}_{6.9} \mathrm{Ni}_{5.6} \mathrm{Be}_{12.5}\right)$ & 1,420 & 1.9 & 2,000 & 8.6 & {$[88]$} \\
$\mathrm{Zr58}\left(\mathrm{Zr}_{58.5} \mathrm{Ti}_{14.3} \mathrm{Nb}_{5.2} \mathrm{Cu}_{6.1} \mathrm{Ni}_{4.9} \mathrm{Be}_{11.0}\right)$ & 1,130 & 1.8 & 1,850 & 12.1 & {$[88]$} \\
$\mathrm{Zr60}\left(\mathrm{Zr}_{60.0} \mathrm{Ti}_{14.7} \mathrm{Nb}_{5.3} \mathrm{Cu}_{5.6} \mathrm{Ni}_{4.4} \mathrm{Be}_{10.0}\right)$ & 1,070 & 1.8 & 1,950 & 17.0 & {$[88]$} \\
Vit. 1 & 1,637 & 1.8 & 1.755 & 2.1 & {$[89]$} \\
\hline
\end{tabular}


Table 4 The thermal properties of the $\mathrm{Zr}_{37.5} \mathrm{Ti}_{32.2} \mathrm{Nb}_{7.2} \mathrm{Cu}_{6.1} \mathrm{Be}_{17.0}$ MGMCs with different withdrawal velocities $[69,95]$.

\begin{tabular}{ccccc}
\hline Withdrawal velocity $(\mathrm{mm} / \mathrm{s})$ & $T_{g}(\mathrm{~K})$ & $T_{x}(\mathrm{~K})$ & $\Delta H_{x}(\mathrm{~J} / \mathrm{g})$ & Microstructure \\
\hline 0.2 & - & - & - & Eutectic + Dendrite \\
0.5 & 604 & 735 & 36.75 & Dendrite + Glass \\
0.8 & 604 & 727 & 57.39 & Dendrite + Glass \\
1.0 & 603 & 727 & 58.09 & Dendrite + Glass \\
1.5 & 604 & 720 & 59.10 & Dendrite + Glass \\
\hline
\end{tabular}


Figure 1 The amorphous structure (a) and the cubic crystalline, and (b) CuZr alloy by computer simulations.

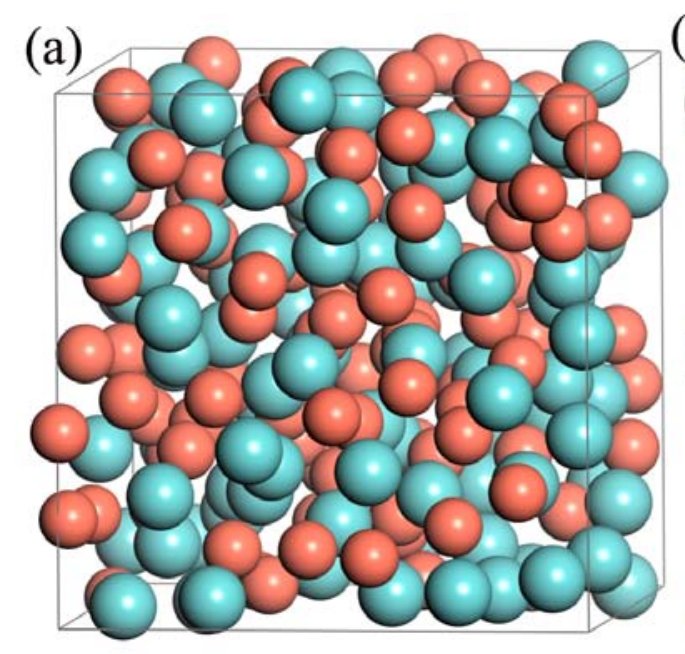

(b)

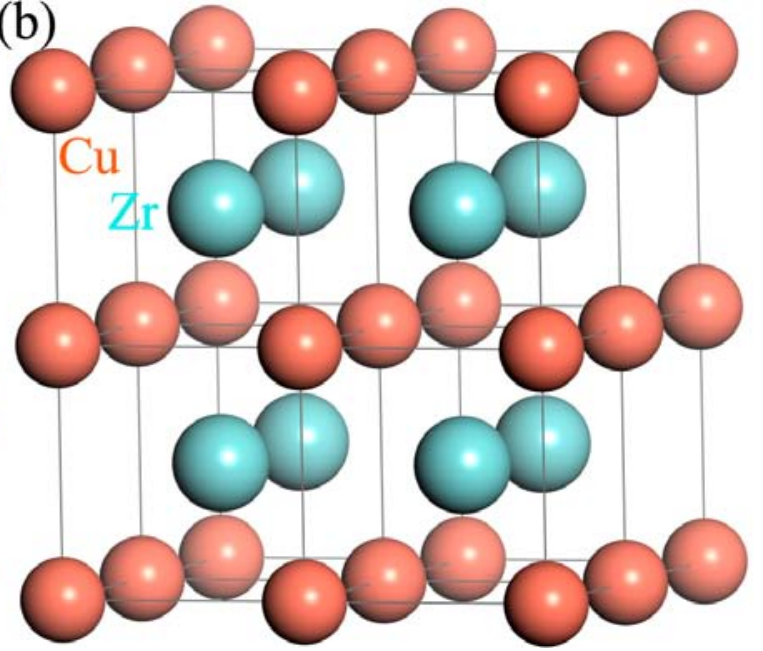


Figure 2 Different BMG systems with the maximum diameters as well as their discovered years, including Nd- [20], Y- [17], Pt- [33], Cu- [15], Ca- [30], Fe- [25], Co[26], La- [34], Mg- [22], Ni- [32], Ti- [29], and Pd-based BMGs [12].

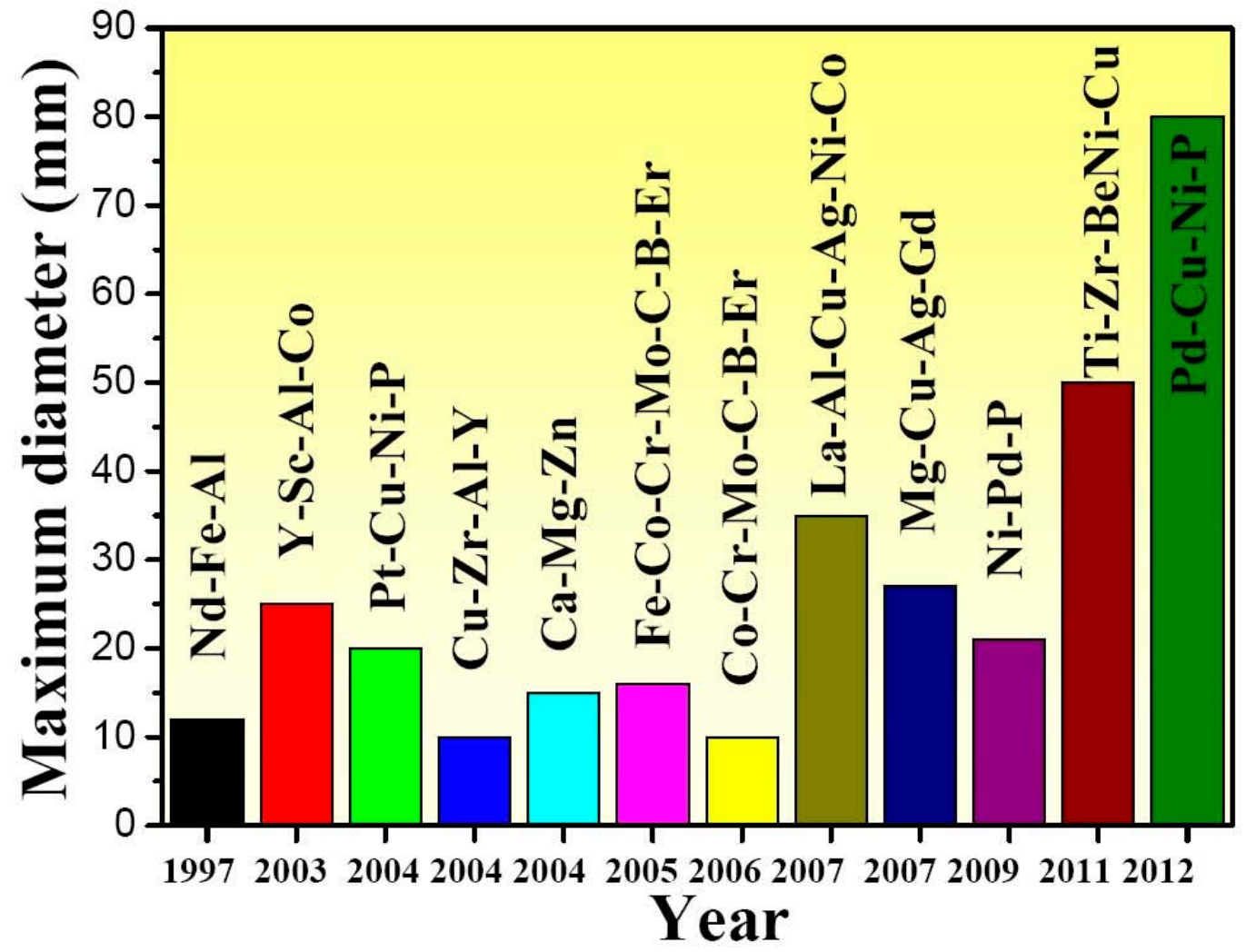


Figure 3 An optical micrograph showing uniformly-distributed WC particles in the V106 matrix (a) [37], X-ray diffraction patterns of the V106 matrix, the alloy reinforced with the 10 vol. \% WC, and pure WC particle (b) [38], and quasi-static compression stress-strain curves of V106 composites reinforced with Ta, W, and WC particles [39].
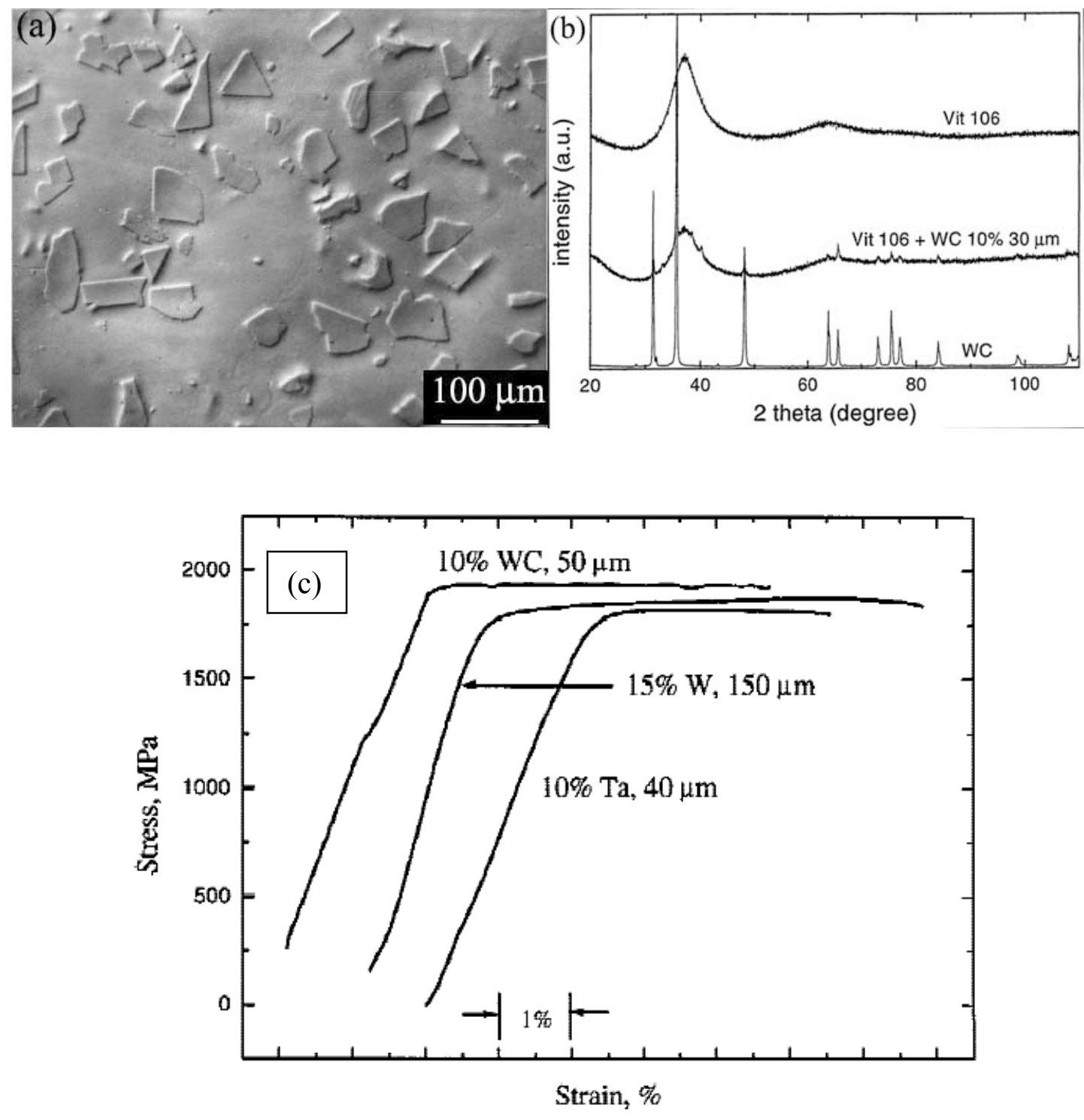
Figure 4 Scanning-electron-micrographic (SEM) image of the transverse cross section of the cast composite alloy containing 40 vol. \% Ti powders [48].

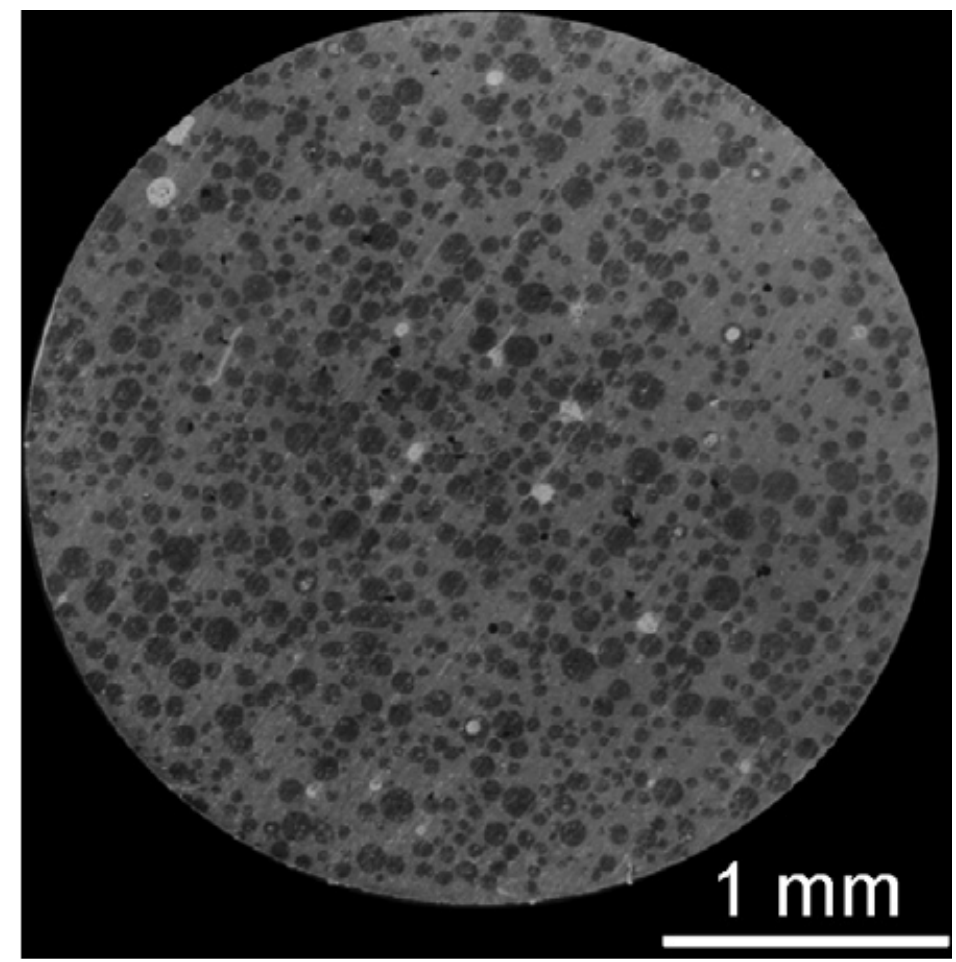


Figure 5 (a) Hardness values of the matrix reinforced by different volumes of the $\mathrm{ZrC}$ particles, and (b) linear fitness of the modulus, E, and yielding strength, $\sigma_{y}$, with increasing the volume fraction $\left(\mathrm{V}_{\mathrm{f}}\right)$ of $\mathrm{ZrC}$ [42].

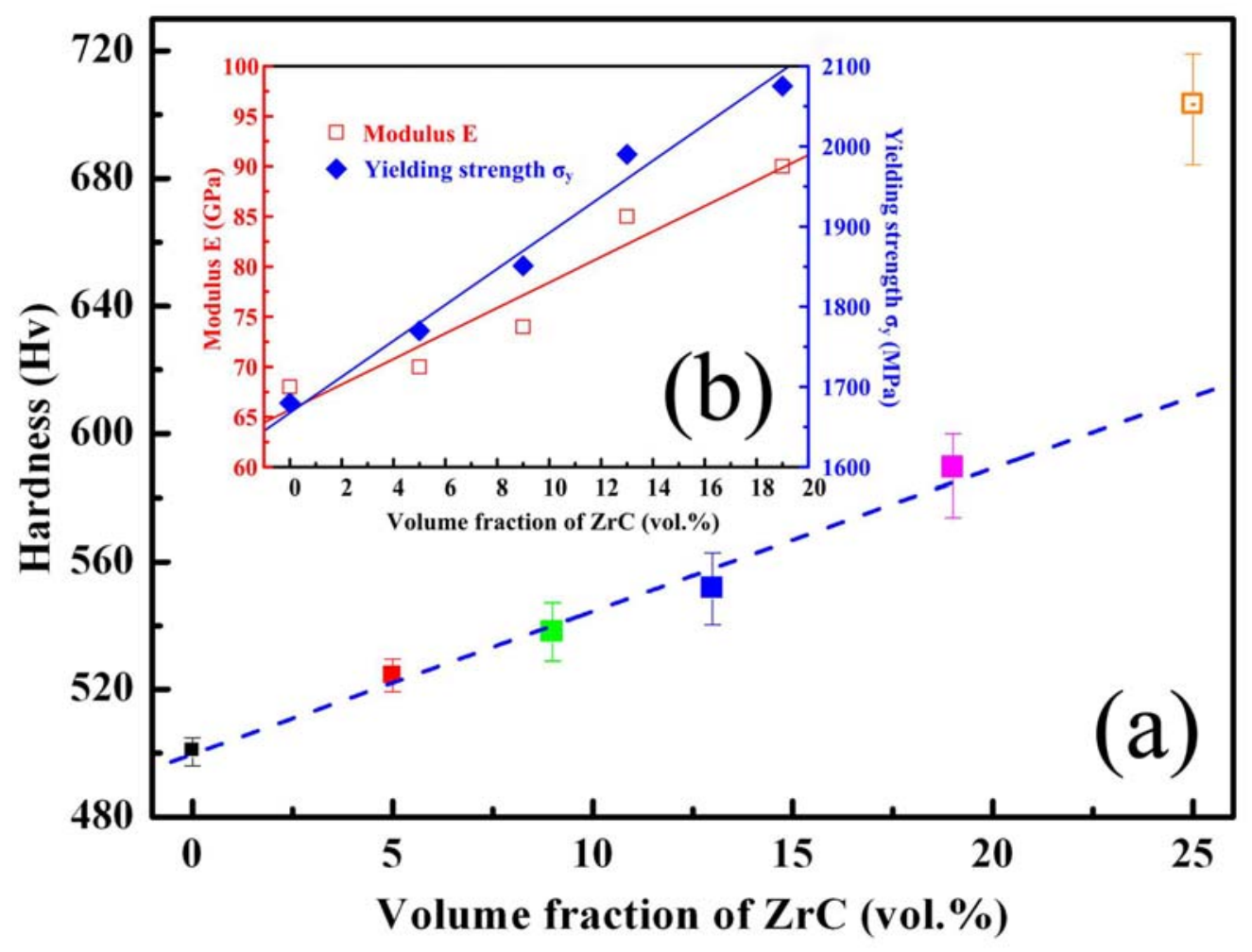


Figure 6 SEM micrograph in the backscatter mode of 80 vol. \% W wire / metallic glass matrix composite. The sample is polished and cut normal to the uniaxial reinforcement $[56]$.

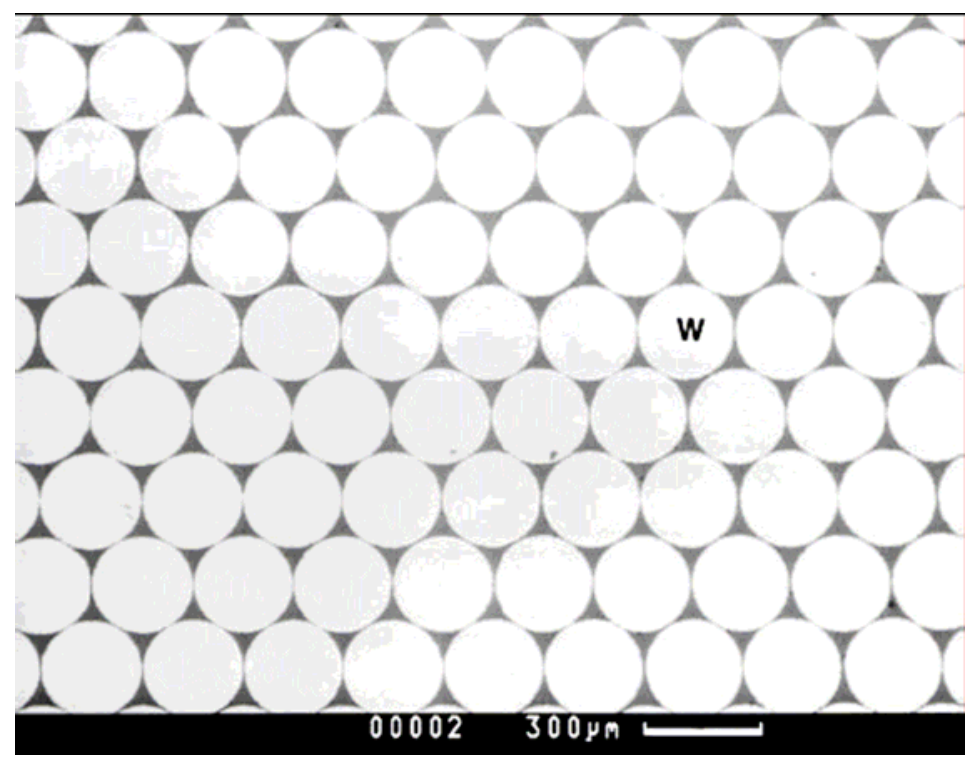


Figure 7 SEM backscattered-electron image of in-situ composite microstructure (Inset: $\mathrm{X}$-ray diffraction pattern for the $\left(\mathrm{Zr}_{75} \mathrm{Ti}_{18.34} \mathrm{Nb}_{6.66}\right)_{75} \mathrm{X}_{25}$ in-situ composite [86].

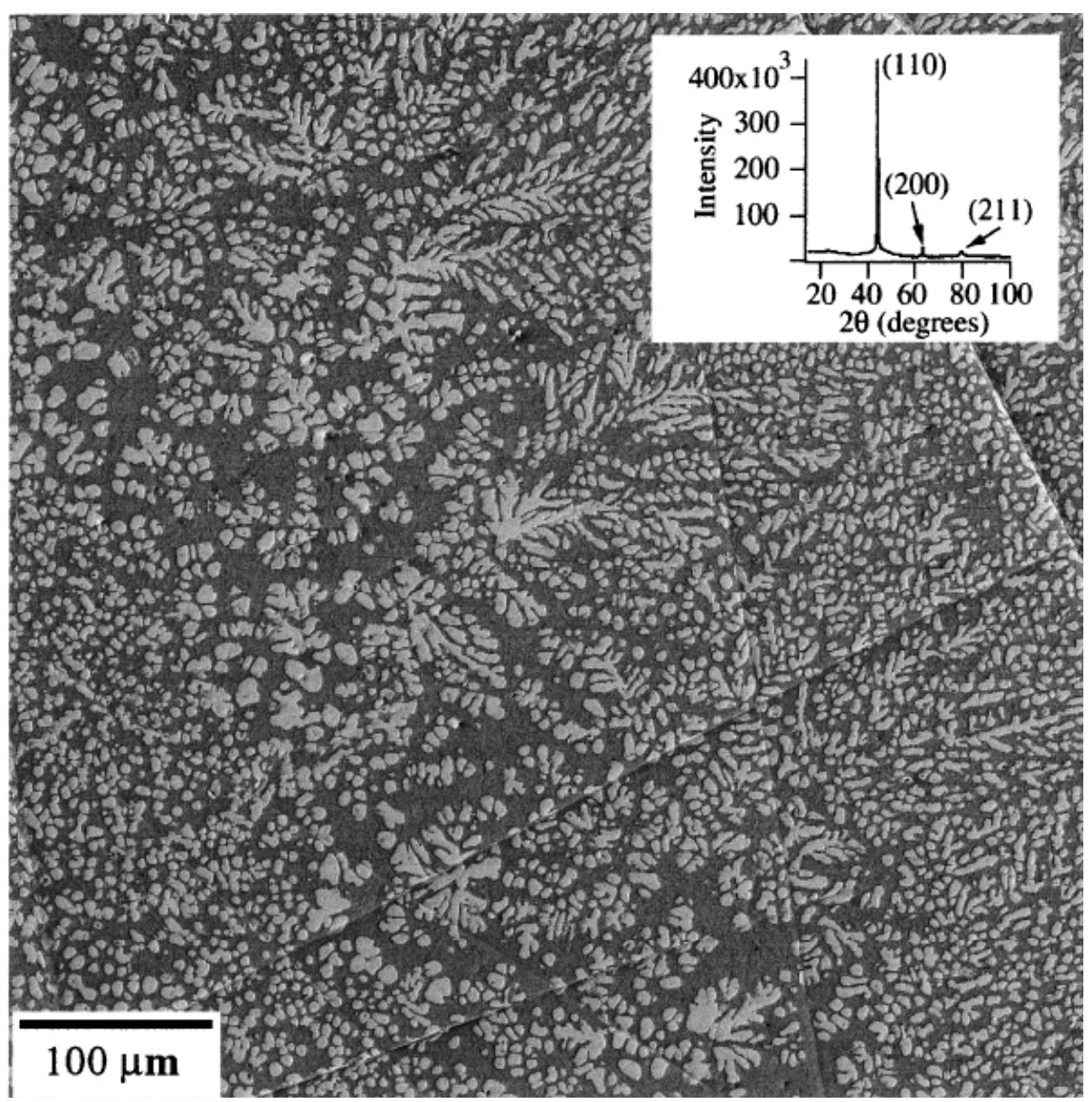


Figure 8 Processing map (a quasi-equilibrium pseudo-binary phase diagram) for in-situ $\beta$-phase-reinforced composites during a cooling experiment. The glass-transition temperature, $T_{\mathrm{g}}$, of the matrix is close to that of Vit $1\left(350^{\circ} \mathrm{C}\right)$. The composites with compositions marked E, F, G, and $\mathrm{H}$ were processed and studied to determine their phase fractions to confirm the phase diagram [87].

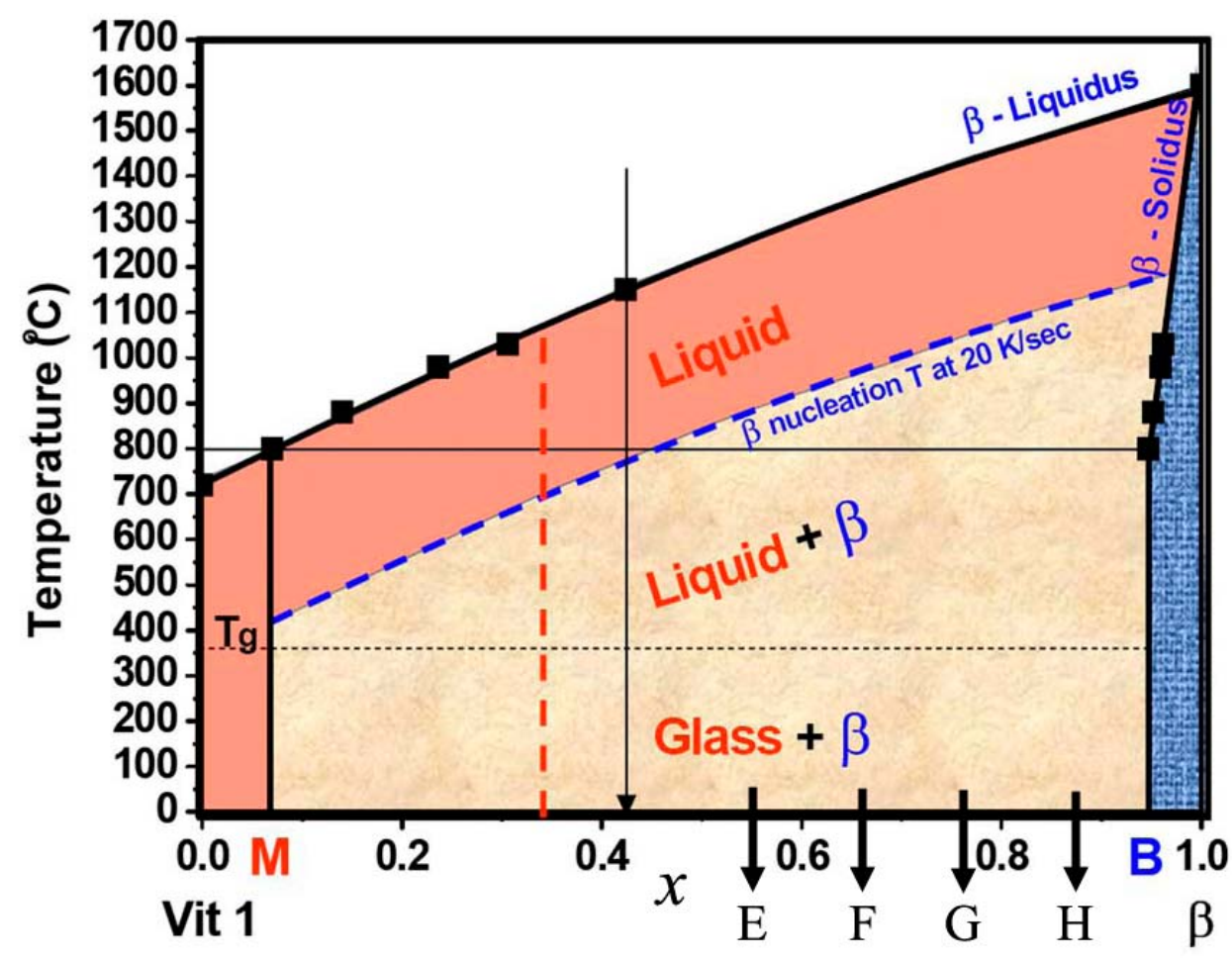


Figure 9 Backscattered-SEM images of the composites (E, F, G, and H) marked in Figure 8 . The light regions designate the $\beta$ phase, and the dark areas are the amorphous matrix. The insets on the upper left corner show the intensity distributions used in estimating the fraction of each phase [87].

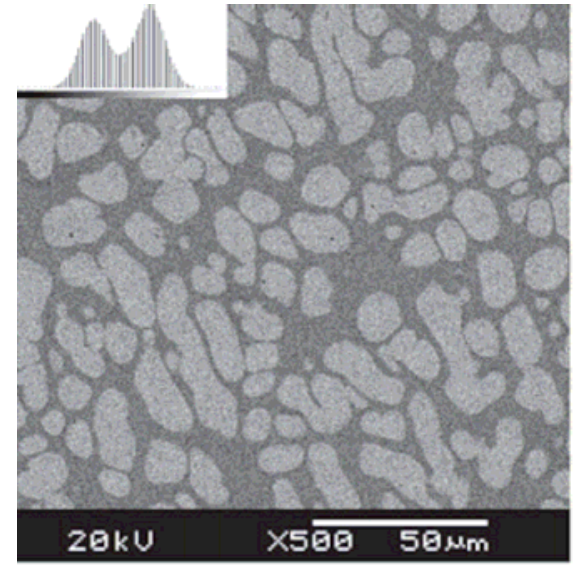

(a) $\mathrm{E}(x=0.55)$

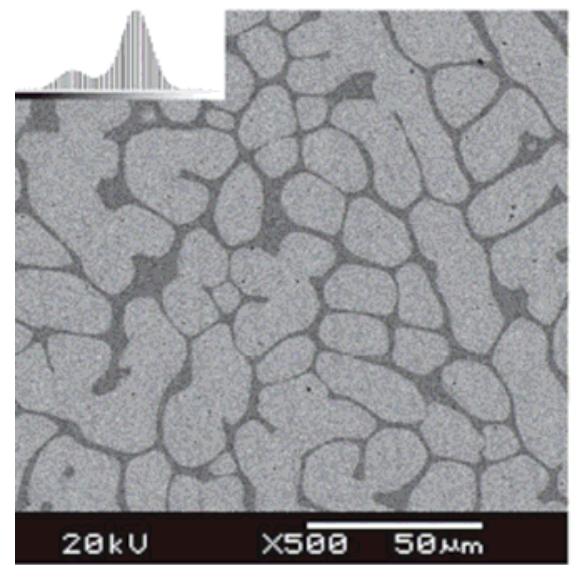

(c) $\mathrm{G}(x=0.77)$

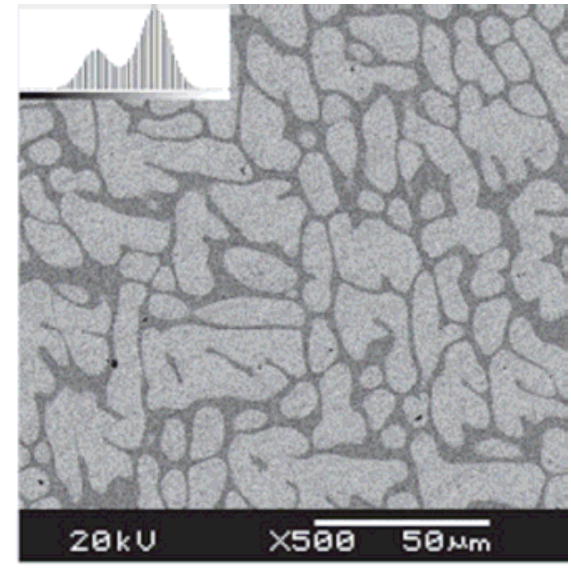

(b) $\mathrm{F}(x=0.66)$

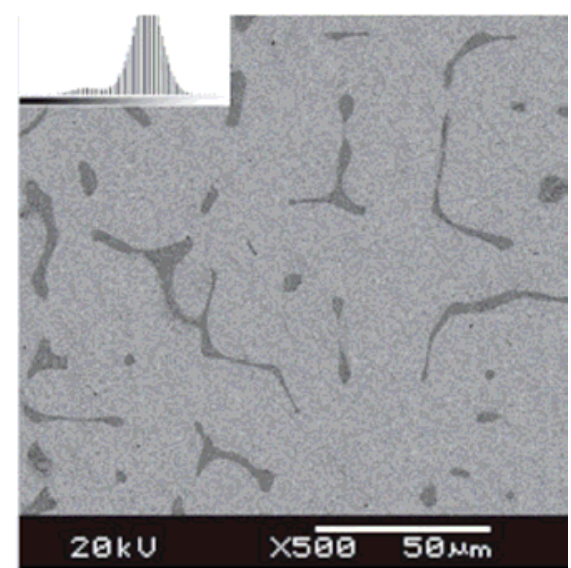

(d) $\mathrm{H}(x=0.88)$ 
Figure 10 A pseudo ternary phase diagram with apexes of zirconium, (titanium + niobium), and $\mathrm{X}$, where $\mathrm{X}$ represents the moiety $\mathrm{Cu}_{5} \mathrm{Ni}_{4} \mathrm{Be}_{9}[88]$.

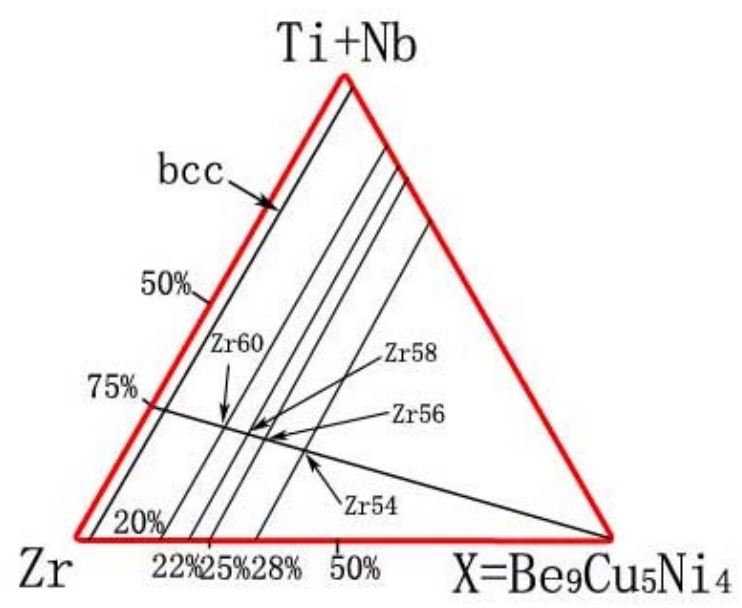


Figure $11 \mathrm{X}$-ray diffraction patterns of four samples with different compositions (labeled as Zr54, Zr56, Zr58, and Zr60, respectively) [88].

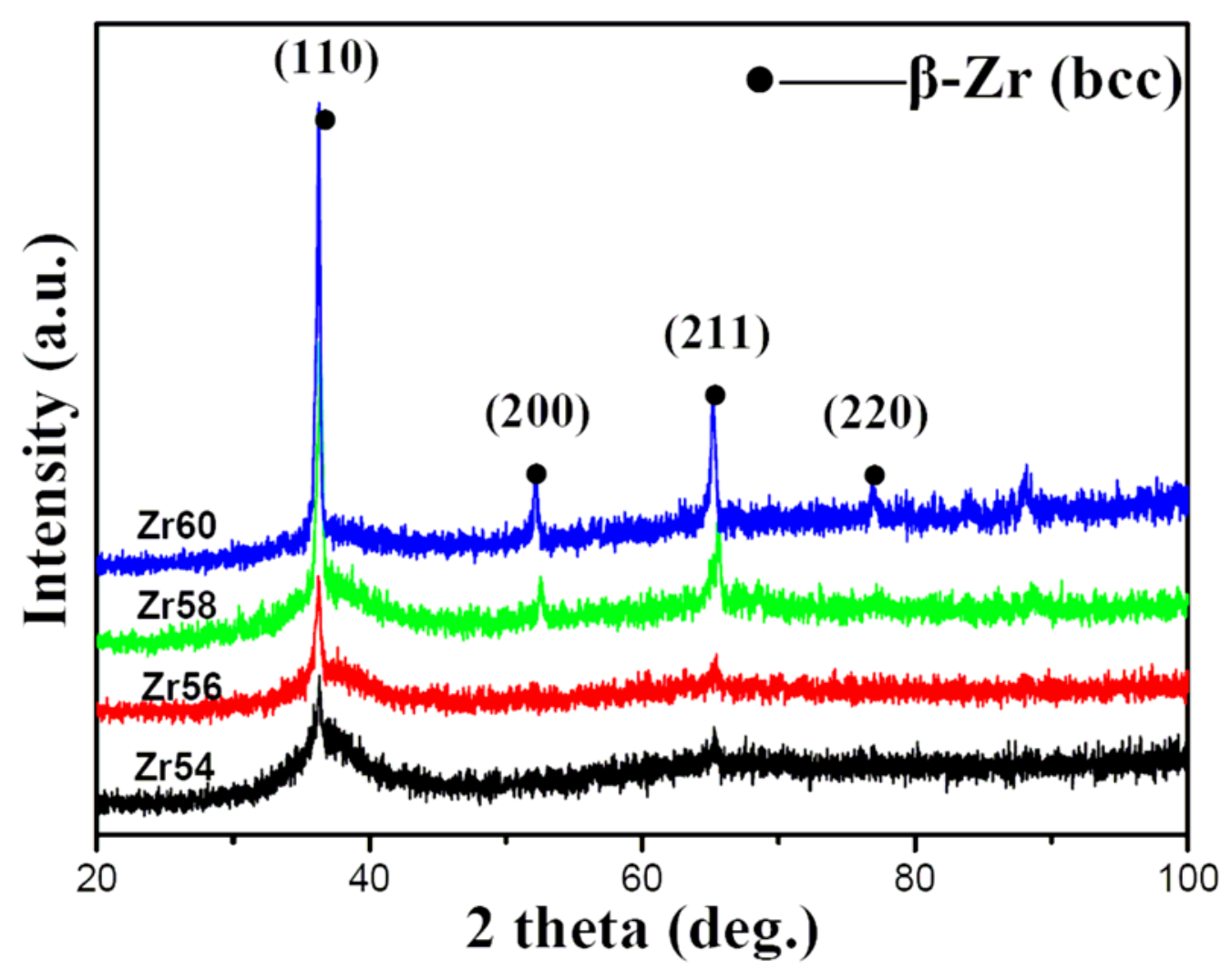


Figure 12 SEM images of the cross sections of the composites with the compositions of (a) Zr54, (b) Zr56, (c) Zr58, and (d) Zr60 [88].

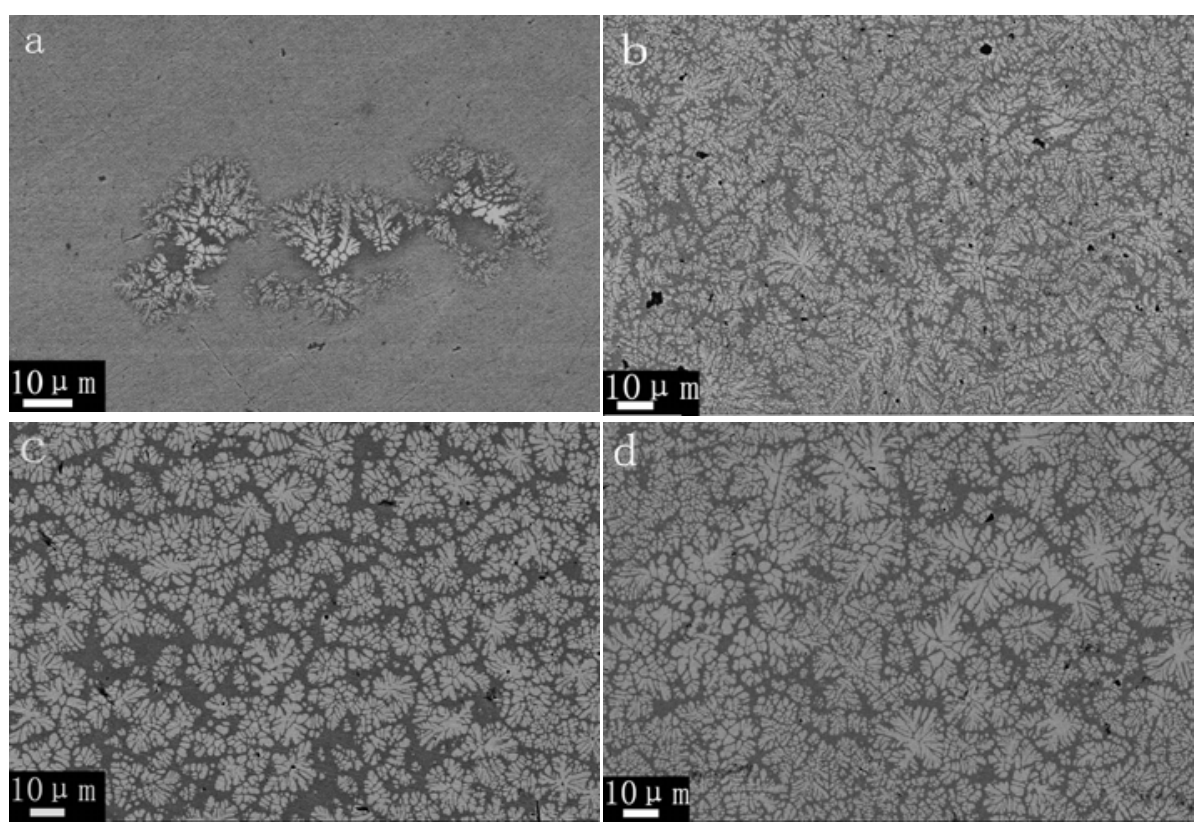


Figure 13 (a) the compressive engineering stress-strain curves of the four composites [88], and (b) the tensile engineering stress-strain curves of the Zr60 composite [90].
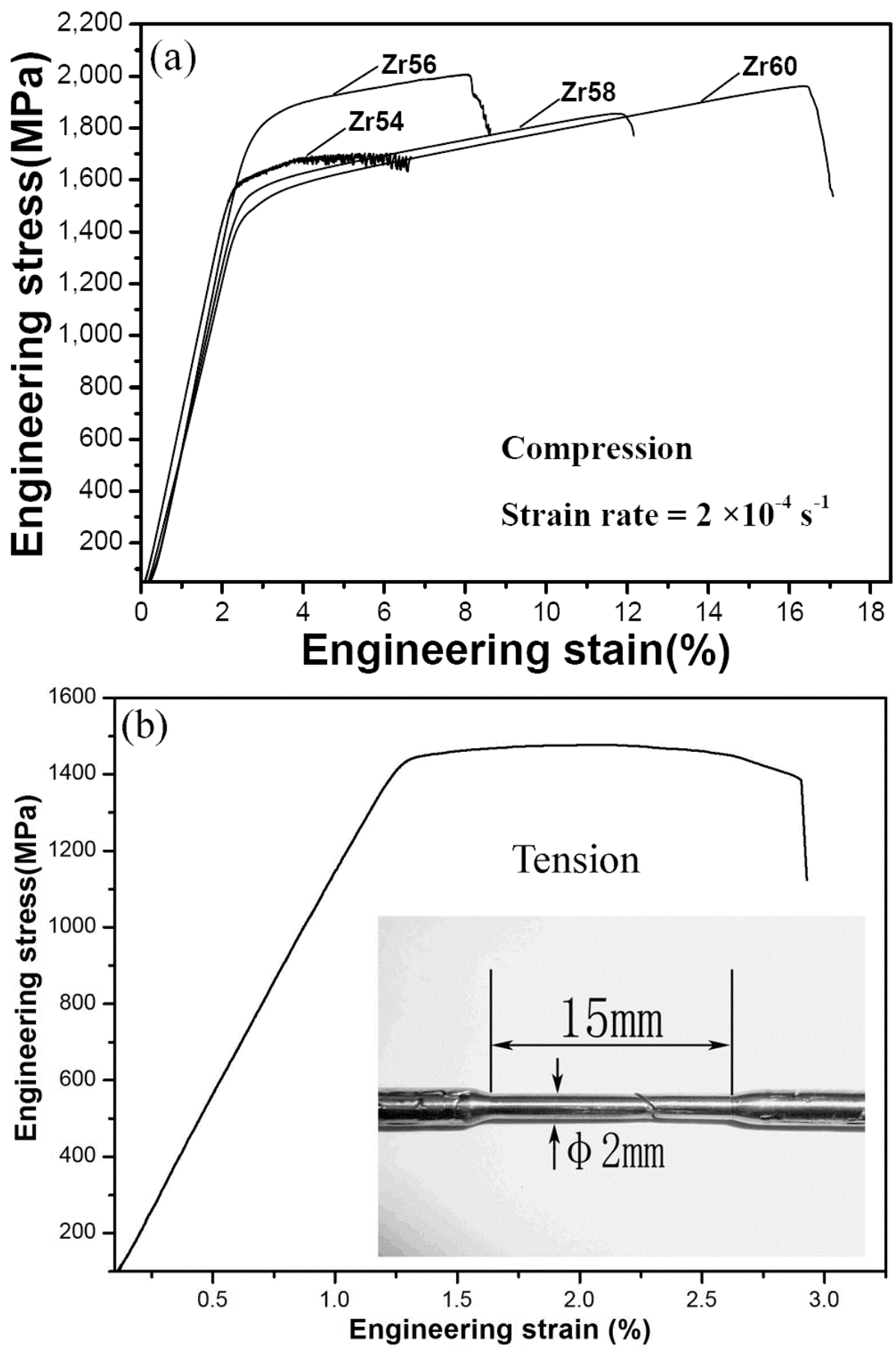
Figure 14 Backscattered SEM of the in-situ composite with a composition of $\left(\mathrm{Zr}_{70} \mathrm{Ni}_{10} \mathrm{Cu}_{20}\right)_{82} \mathrm{Ta}_{8} \mathrm{Al}_{10}$ [113].

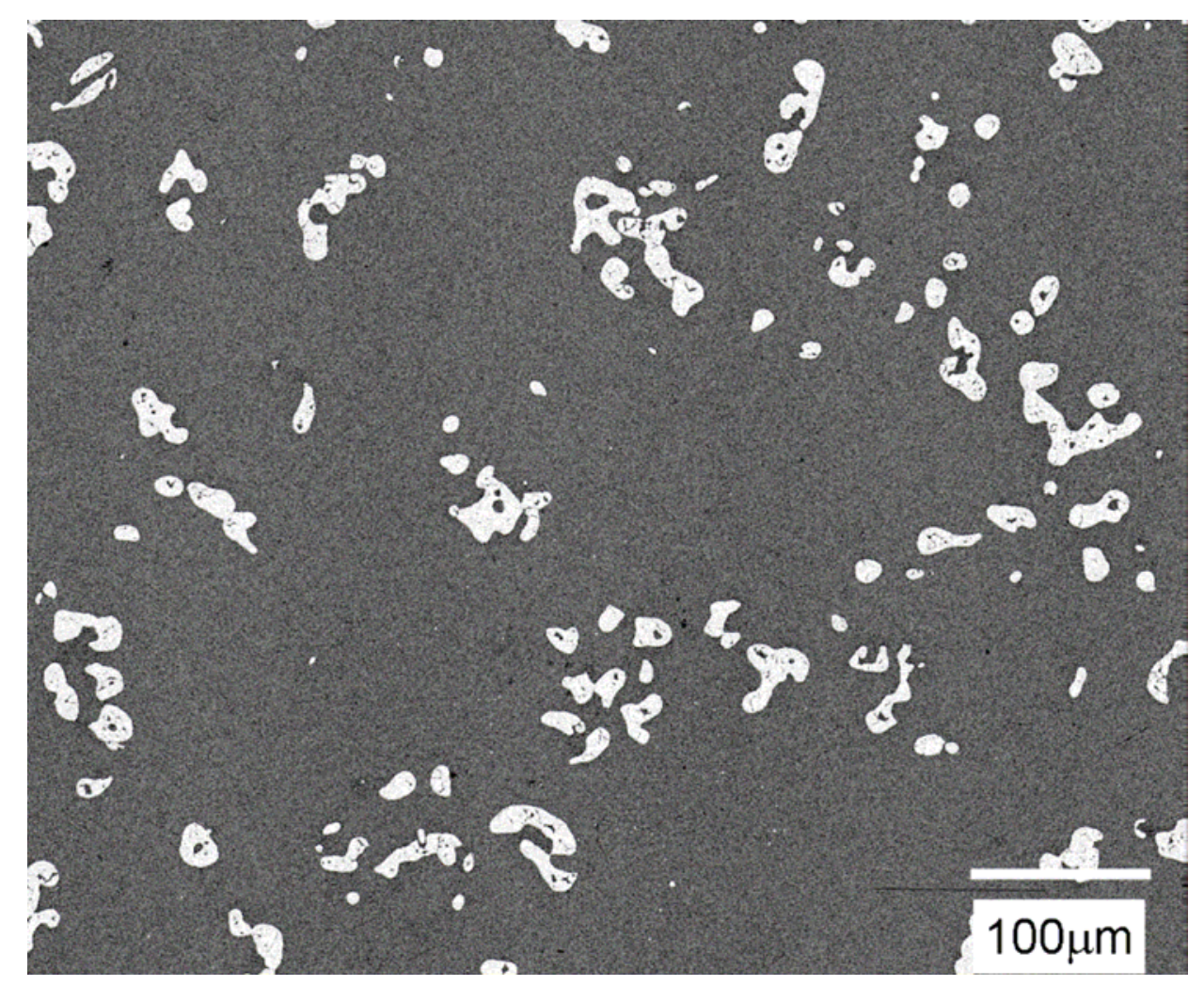


Figure 15 The crystalline structures of the $\mathrm{B} 2 \mathrm{CuZr}, \mathrm{P} 2{ }_{1} / \mathrm{m}$, and $\mathrm{Cm}$ phases $[136,137]$.

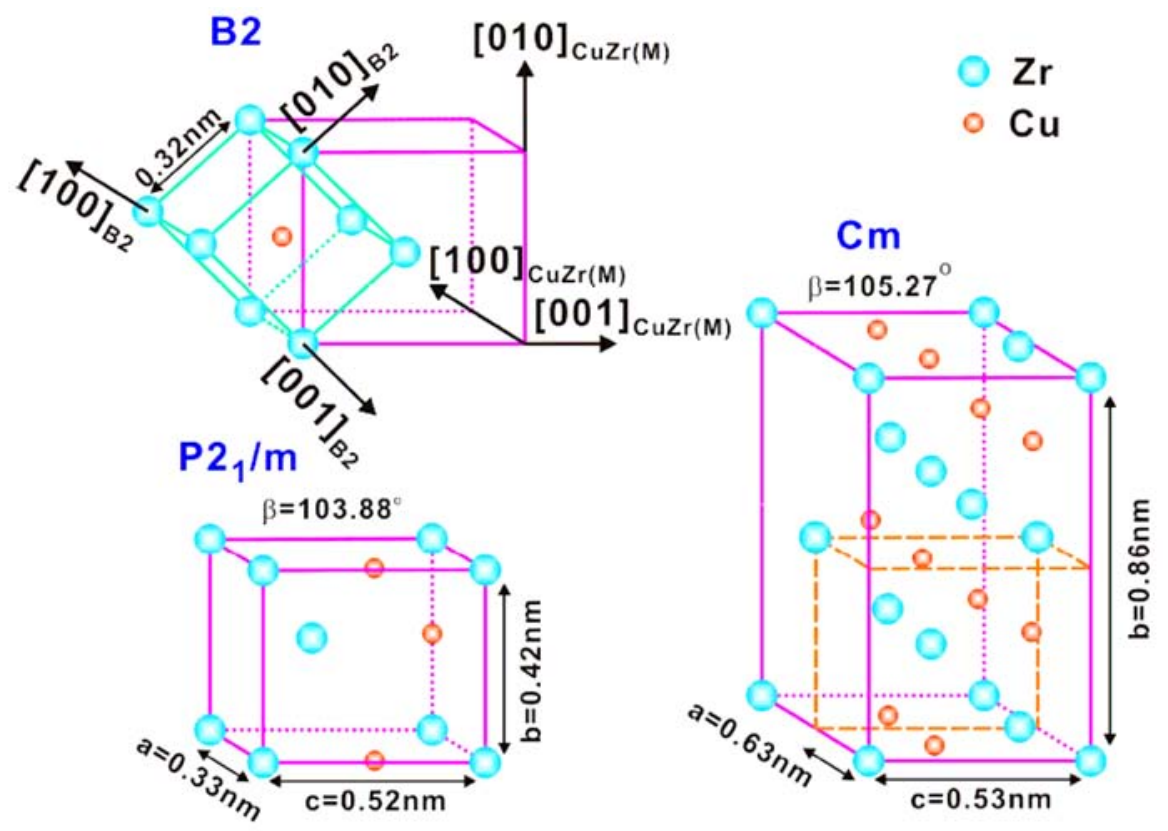


Figure 16 Schematic CCT diagram during the quenching process illustrating the formation of CuZr-based BMG composites [137].

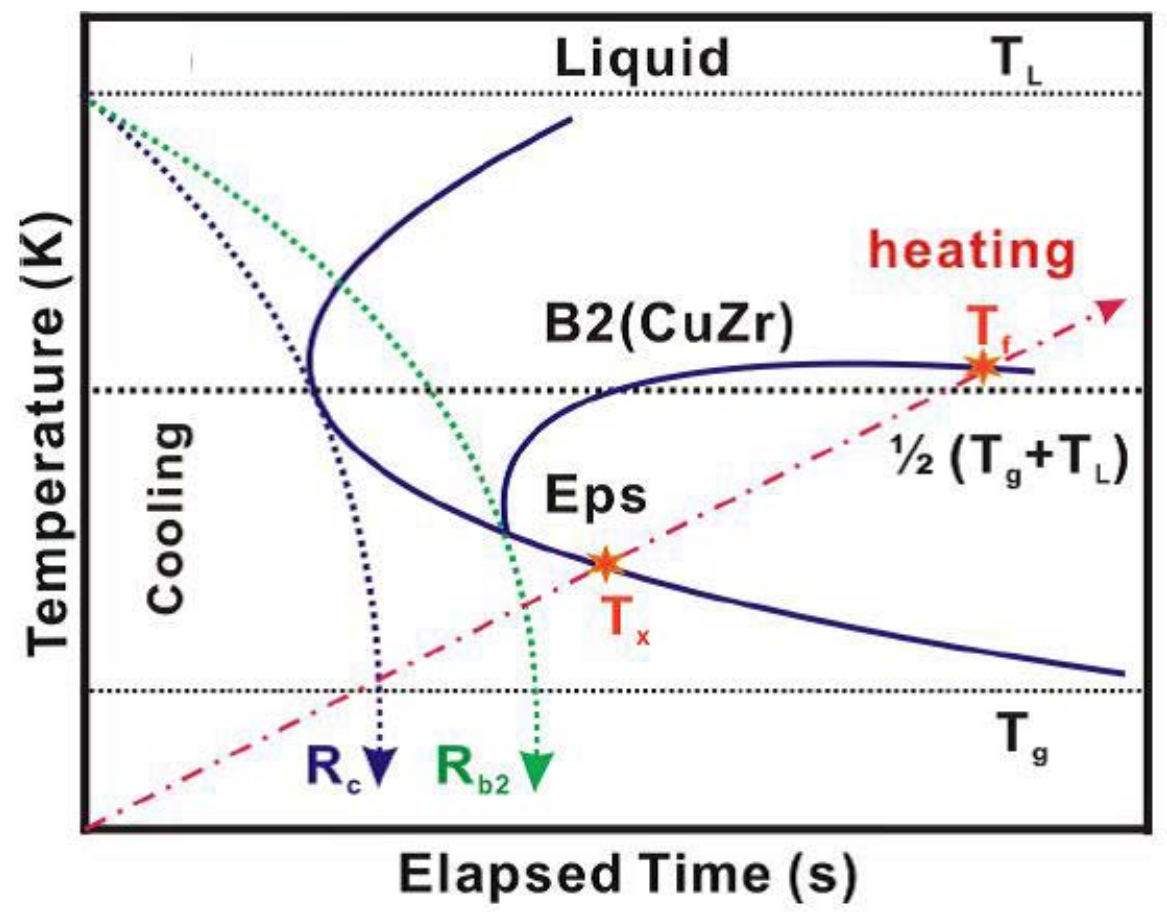


Figure 17 Dependence of tensile ductility on crystalline volume fraction [132].

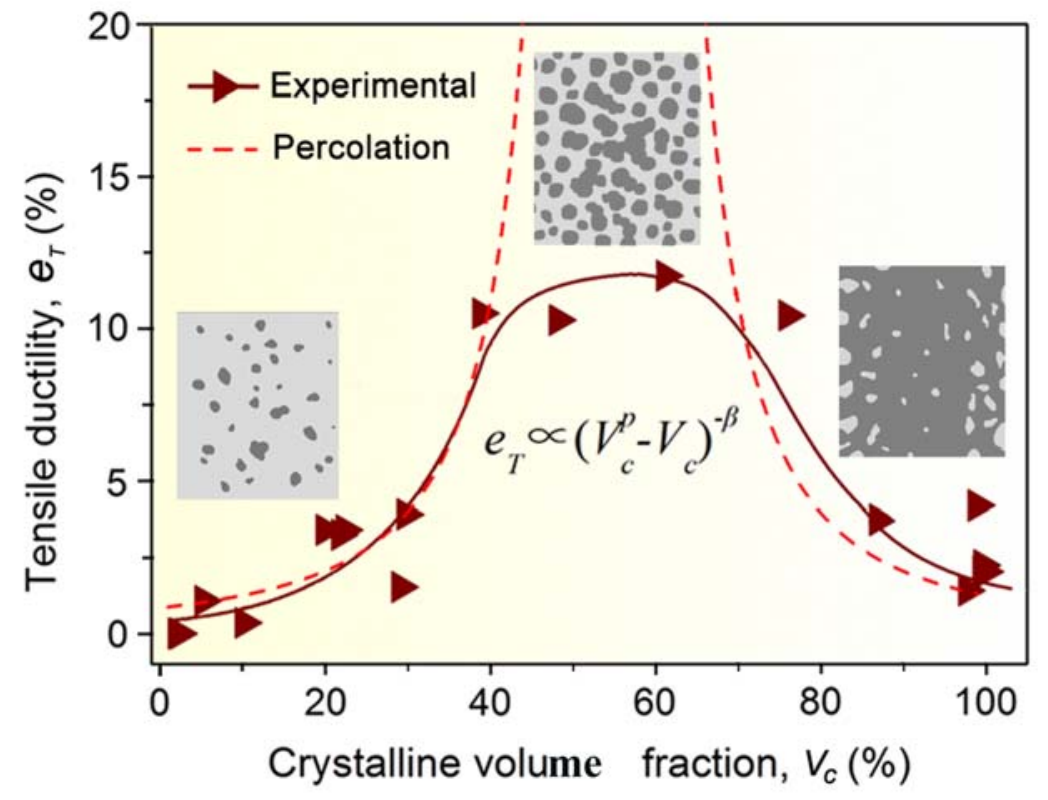


Figure 18 Typical schematic illustration of copper-mold-suction casting, and the arc melter at The University of Tennessee shown in the inset [141].

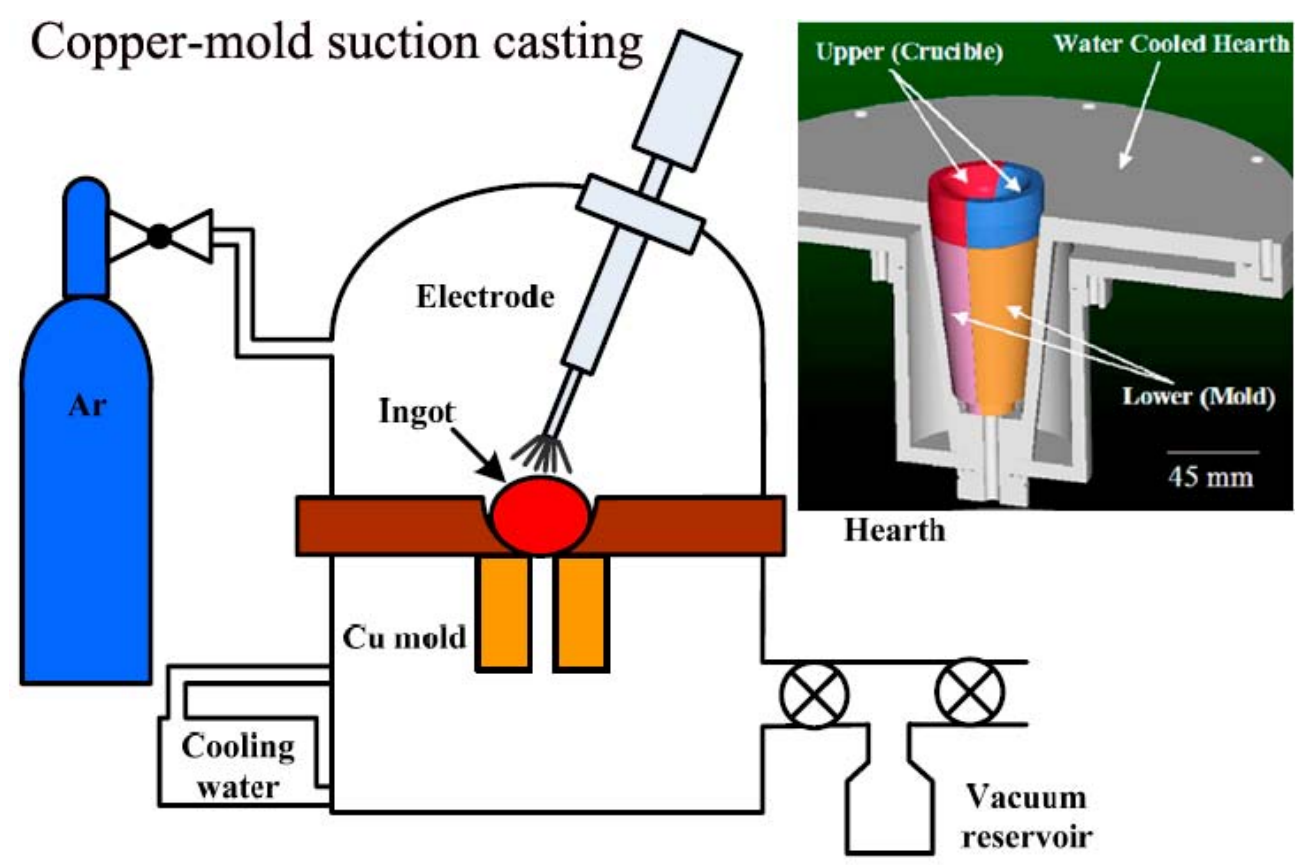


Figure 19 Schematic diagram of the apparatus for casting bulk MGMCs [37].

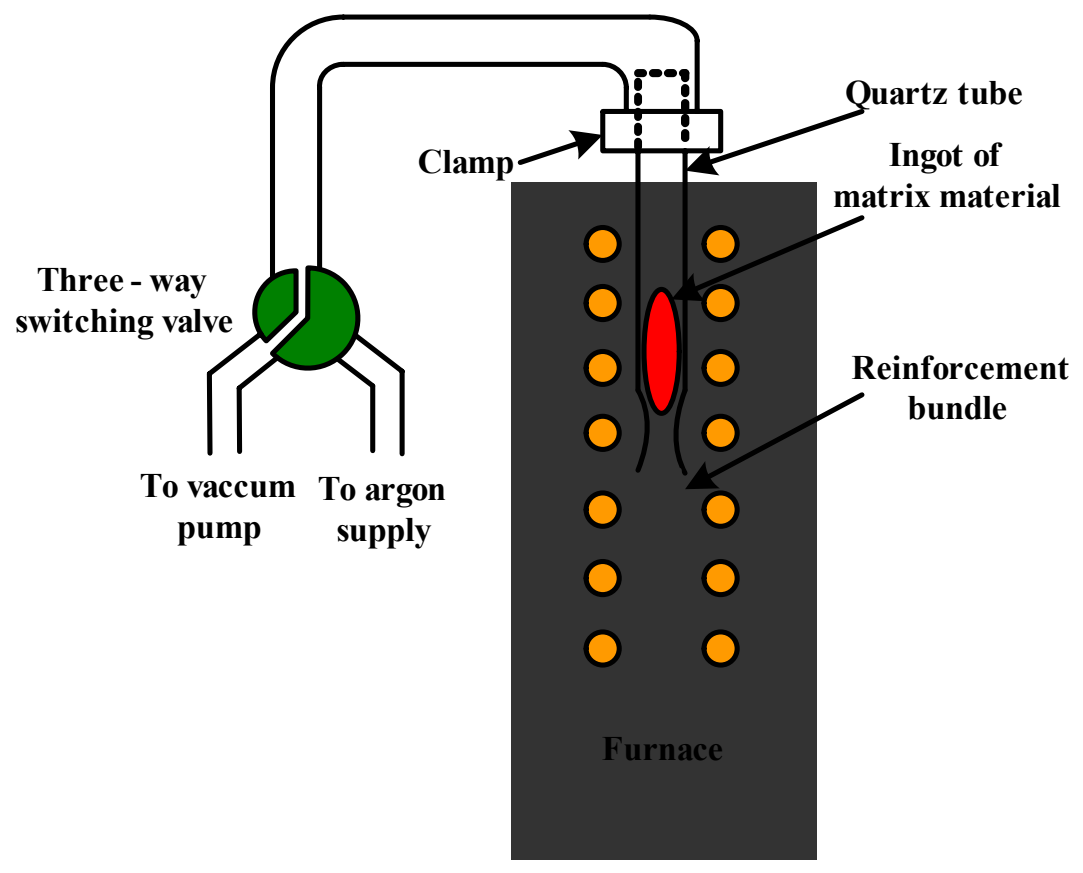


Figure 20 (a) diagram of a water-cooled copper boat used for semisolid processing of in-situ metallic glass matrix composites, and (b) example of a custom coil on a water-cooled copper boat [165].
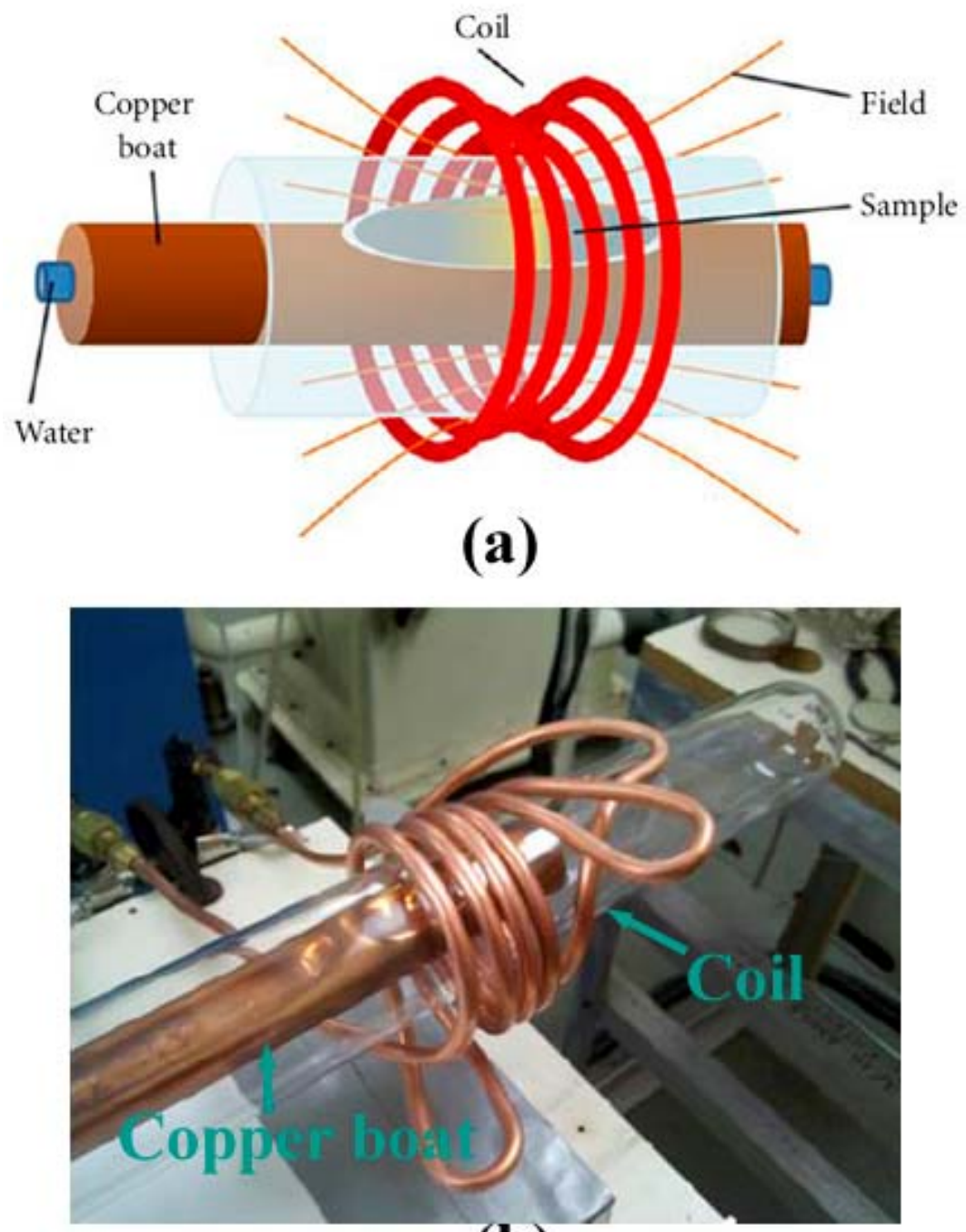

(b) 
Figure 21 Plot of shear modulus versus volume fraction of dendrites for the alloy, DH1, its glass matrix and its dendrite (a) [94] and contrast adjusted SEM of the dendrite structure in a bulk metallic glass matrix composite [165].

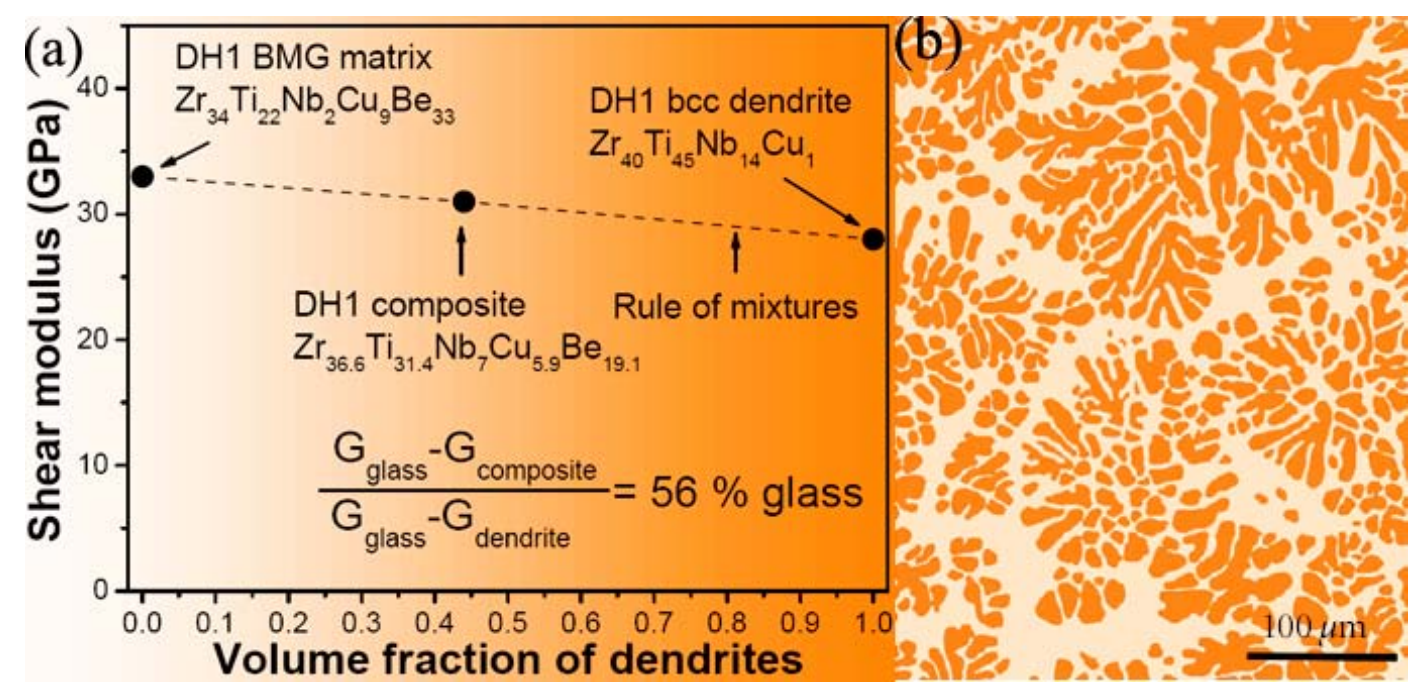


Figure 22 The Bridgman solidification apparatus used by Qiao et al. (a), and the corresponding illustration exhibited in (b).
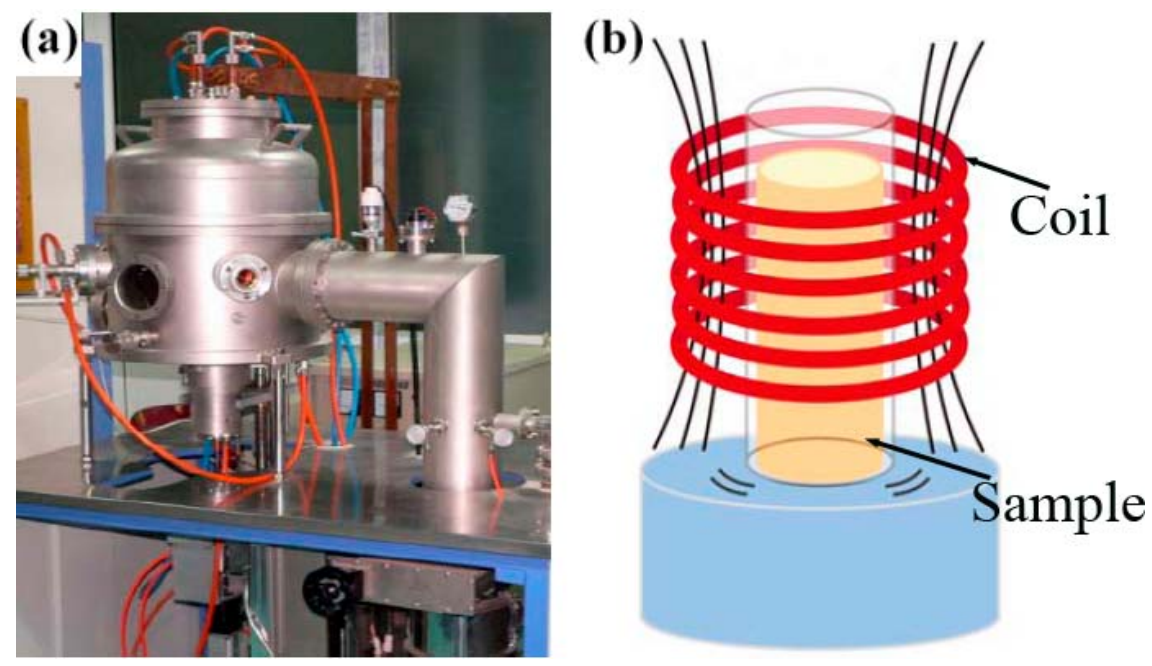
Figure 23 XRD patterns (a), and DSC traces (b) of the composites synthesized with different withdrawal velocities [69].
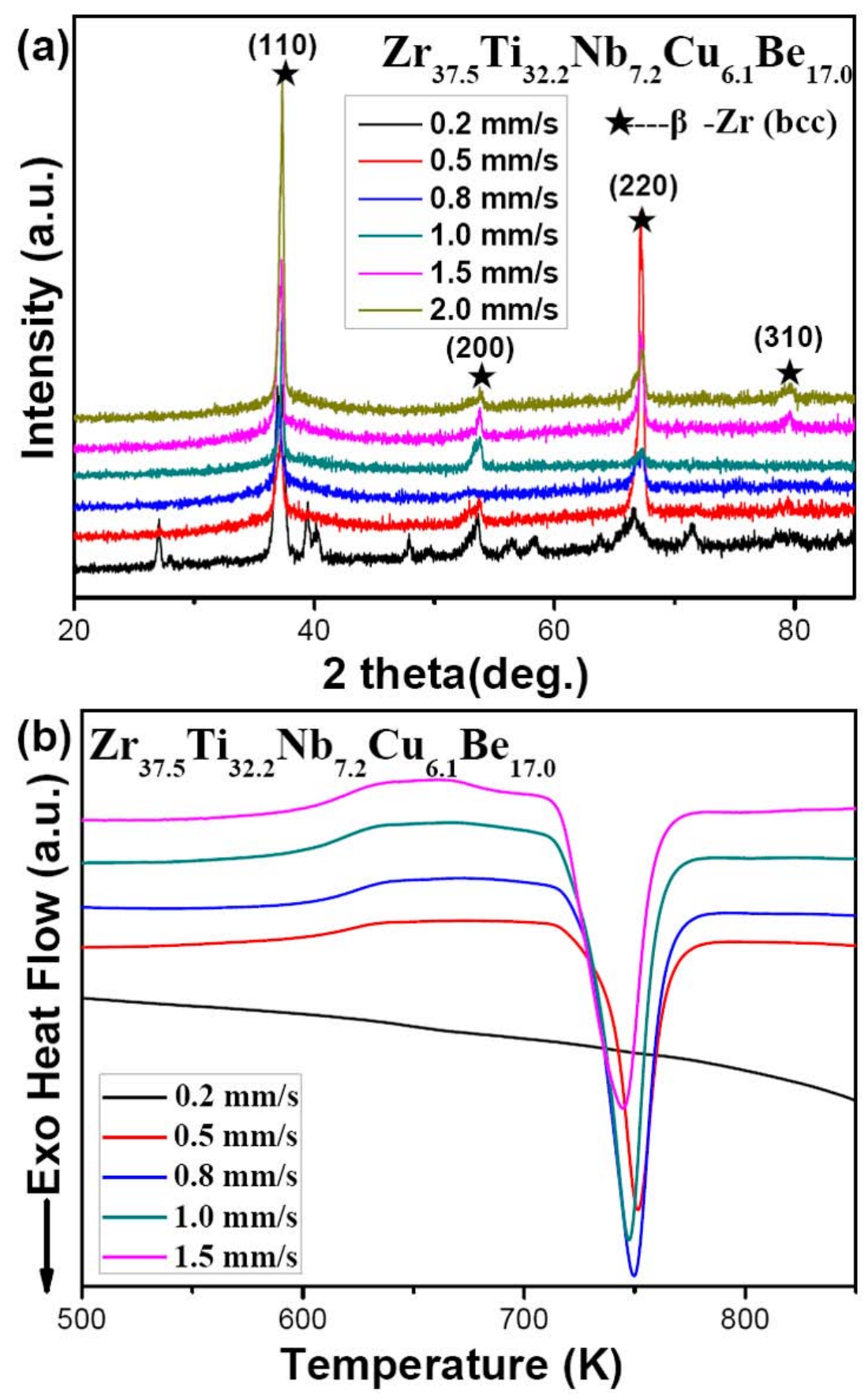
Figure 24 The microstructure of the sample with $\mathrm{v}=1.0 \mathrm{~mm} / \mathrm{s}$ is illustrated in (a) [95], the dependences of spanning lengths of individual dendrite trees on varied withdrawal velocities shown in (b) [95], and the dependences of fracture strengths and plastic strains of the composites developed by the Bridgman solidification on the v (c), the inset in (c) indicating the sample with $\mathrm{v}=1.0 \mathrm{~mm} / \mathrm{s}$ after bending. 

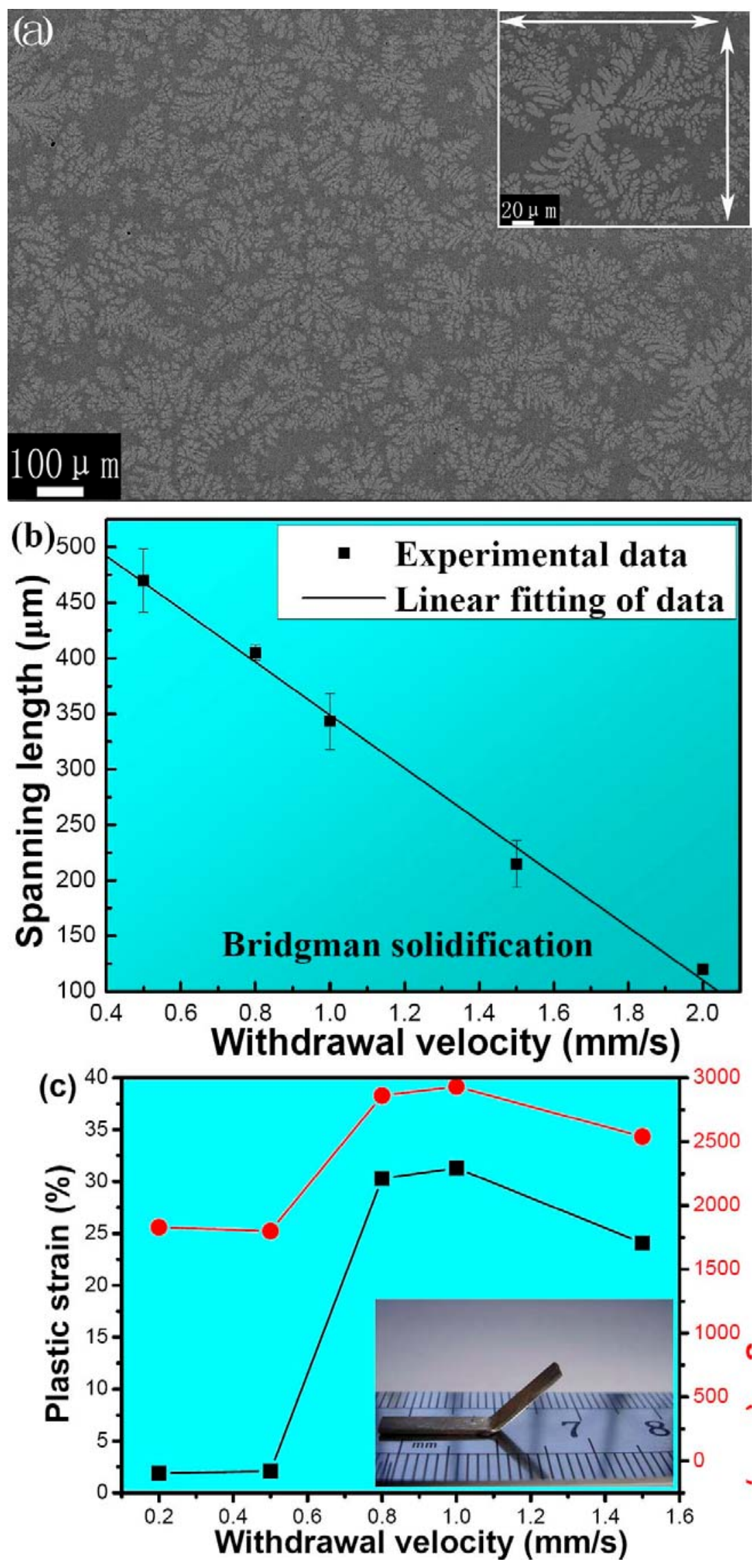
Figure 25 Classification of MGMCs

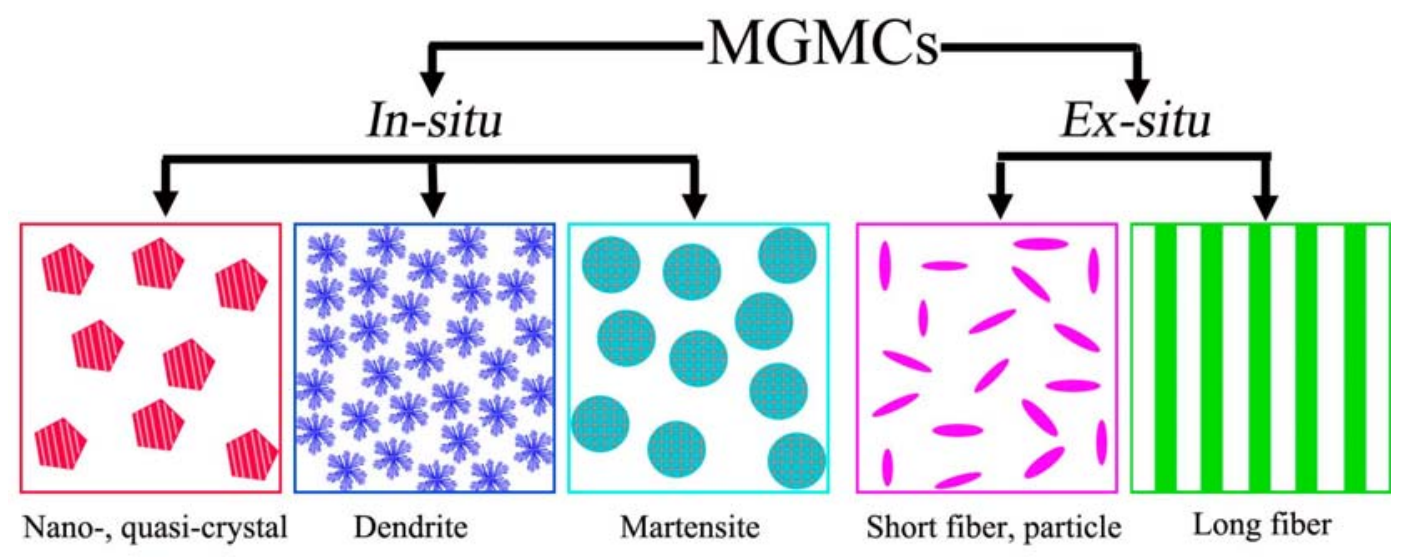


Figure 26 A schematic of the free-volume model in metallic glasses.

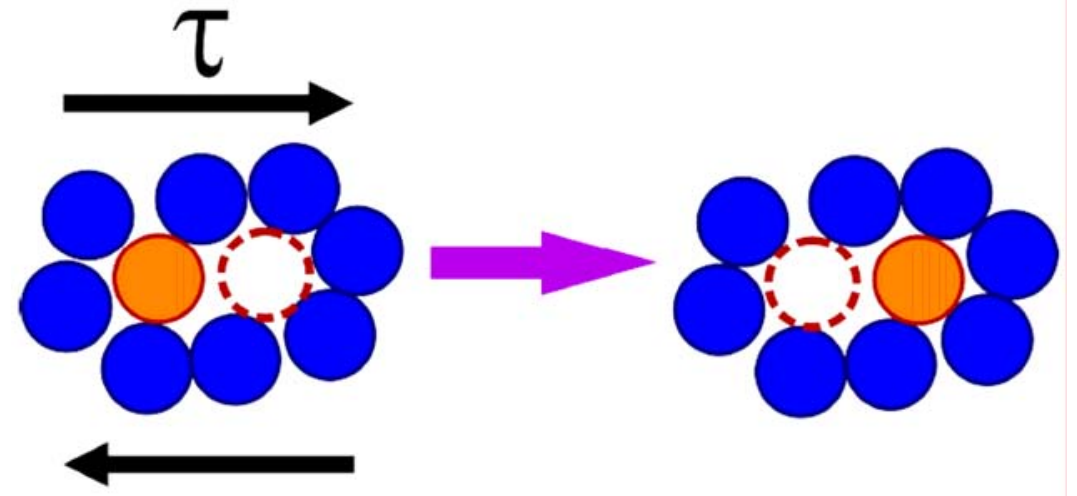


Figure 27 (a) AFM image of the nanoscaled-periodic corrugation in a Vitreloy 1 metallic glass [210], and (b) Periodic corrugation forms by the occurrence of the local quasi-cleavage due to TTZs near the tip; coalescence of the local quasi-cleavage zone with the crack yields a longer crack [209].

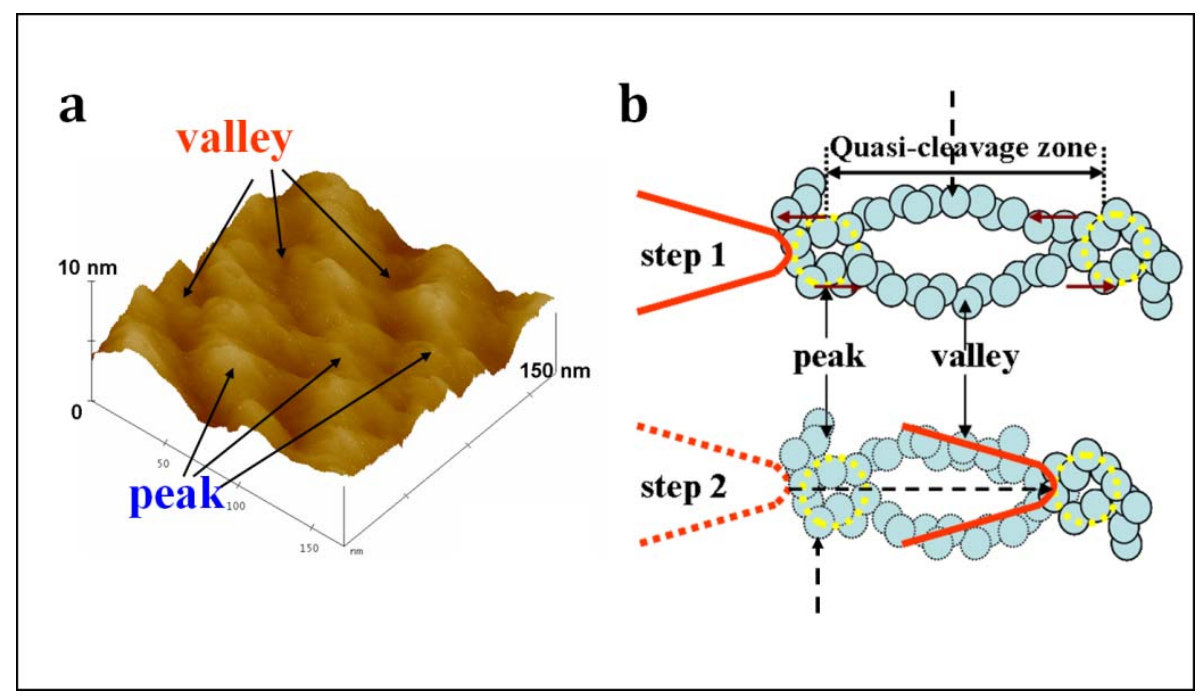


Figure 28 Schematic diagram of the ductile-to-brittle transition mechanism of metallic glasses during fracture. (a) If the curvature radius of the crack tip is greater than the critical wavelength of the meniscus instability, a ductile fracture will occur in the fracture-process zone, where the atomic-cluster motion via shear transformation zones (STZs), as shown in the inset; and (b) in the opposite case, the quasi-brittle fracture through tension-transformation zones (TTZs) [see the inset] is possible ahead of the sharp crack tip [217].

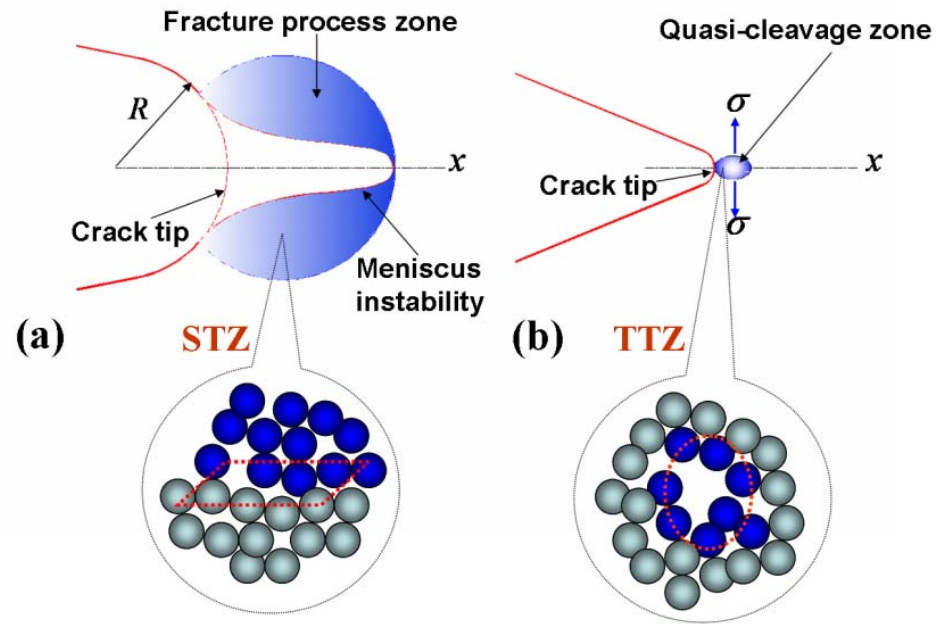


Figure 29 Correlations between the evolution of liquid-like zones, deformation map, relaxation spectrum and energy landscape are established during the glass-to-liquid transition [235].

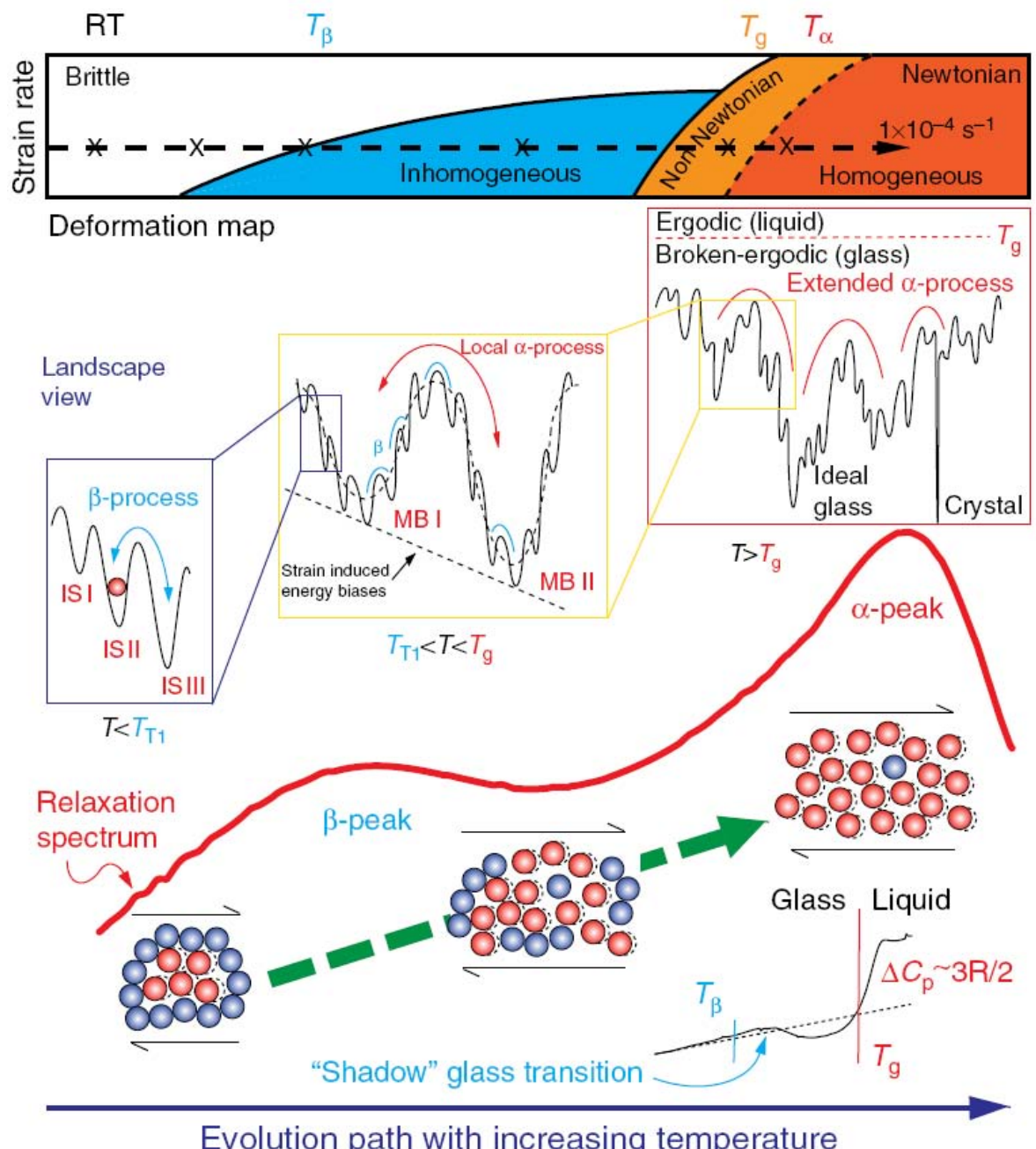

Evolution path with increasing temperature 
Figure 30 Schematic diagrams of the stick-slip model for a single sample where only a dominant shear band formed (a), and several samples tested simultaneously (b).
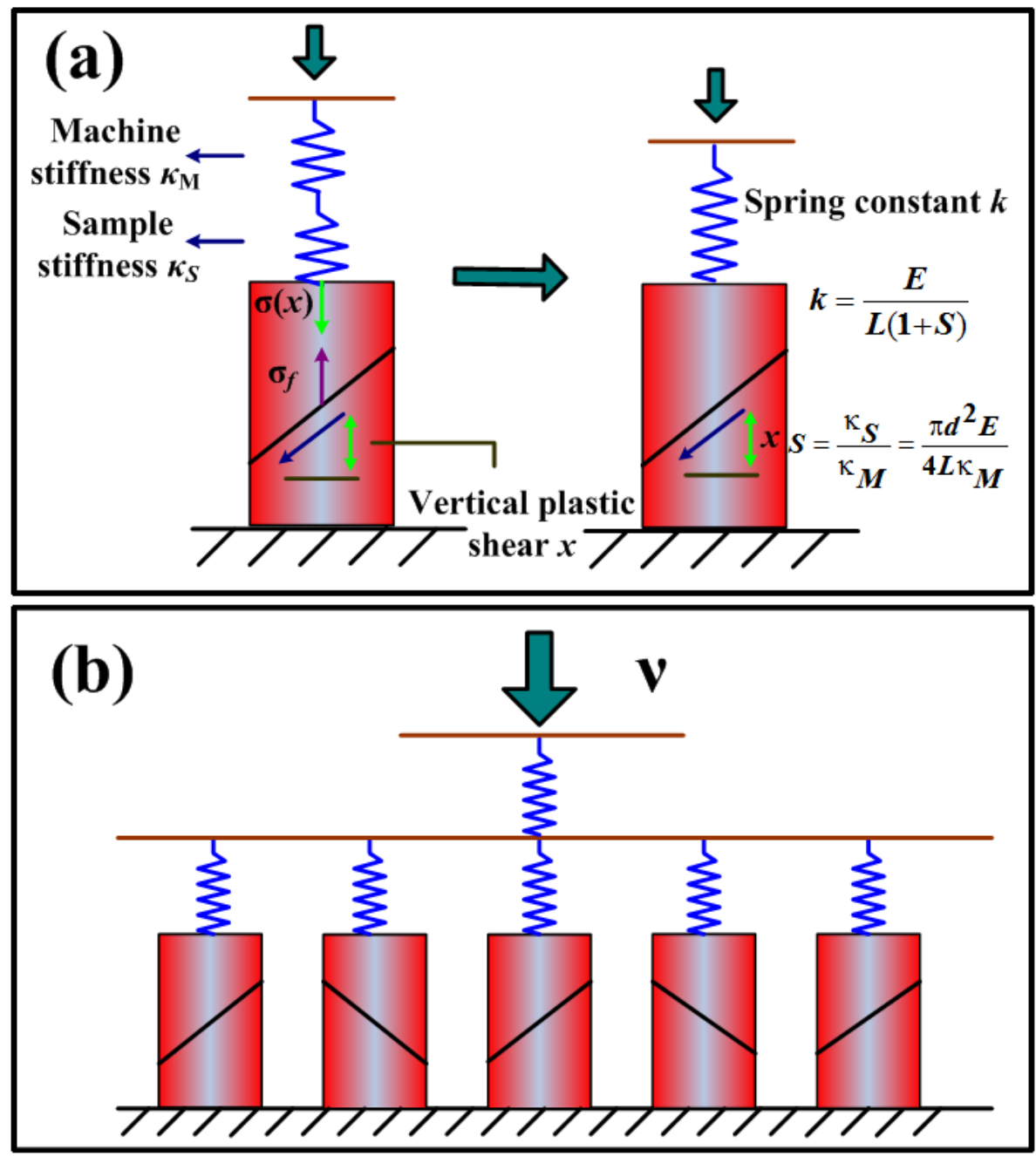
Figure 31 The relationship among the fracture strength, $T_{\mathrm{g}}$, and molar volume for a variety of BMGs [269].

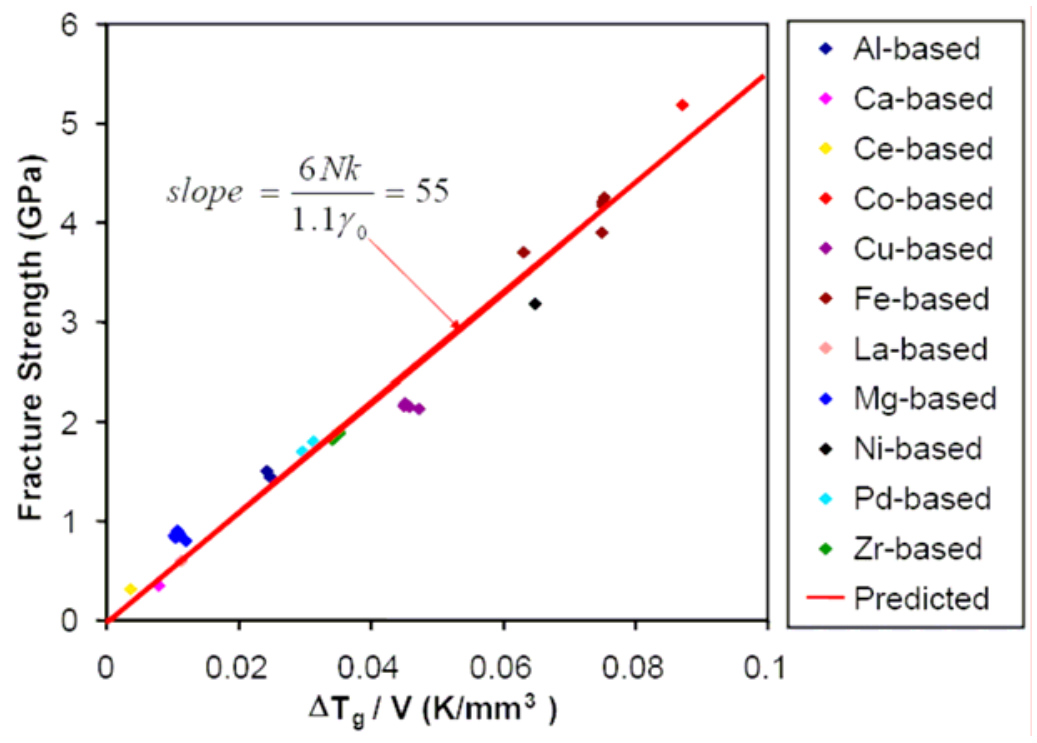


Figure 32 The SEM images of the S2 composite shown in (a), and the quasi-static compressive stress-strain curves of LM1, S1, and S2 (b) [66].
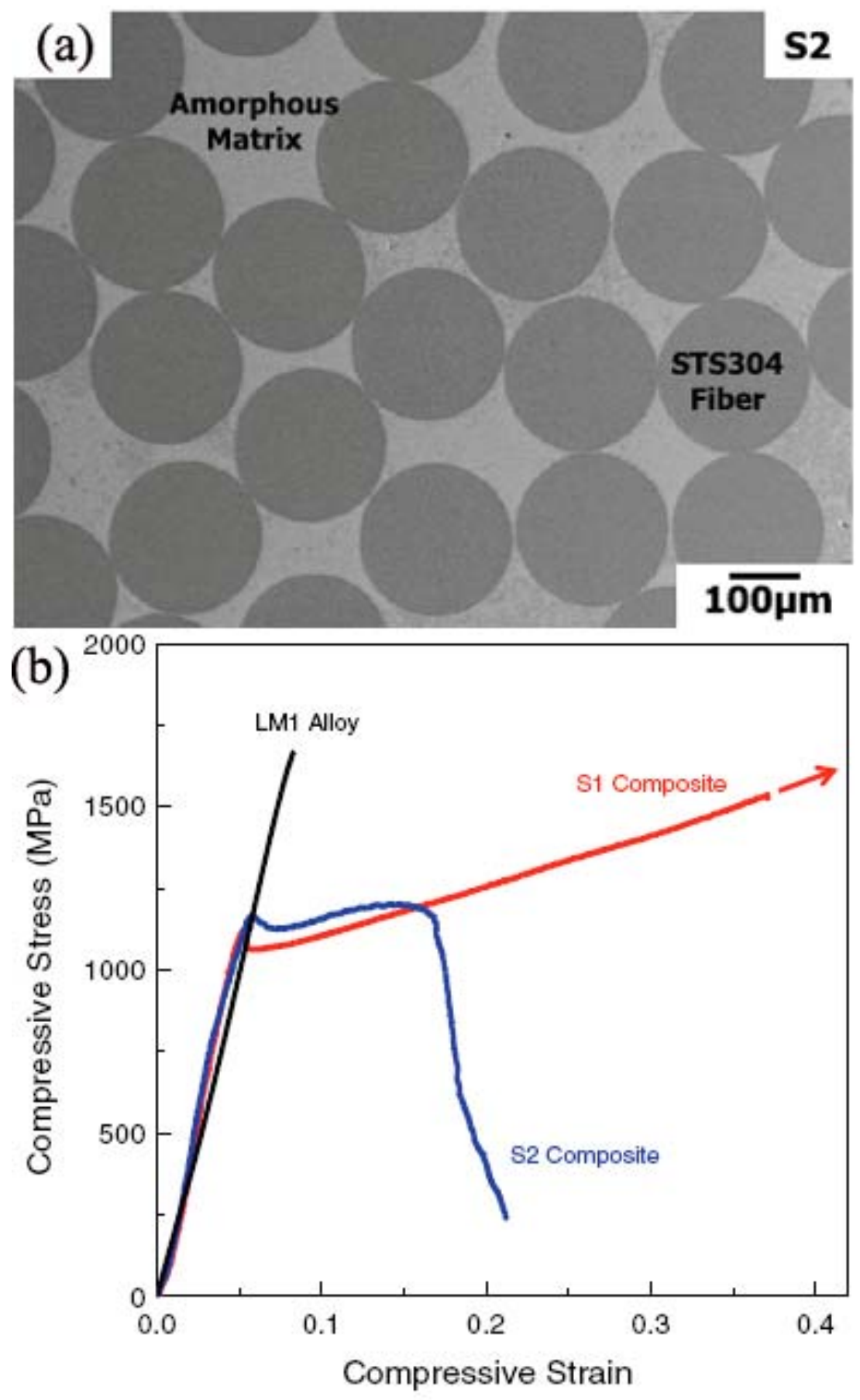
Figure 33 Illustration of the competitive process between the shear and splitting fracture for the tungsten composite at room temperature: (a) variations of shear and splitting fracture strengths with fiber volume fractions, and (b) critical compressive fracture lines for shear and splitting fracture modes of the composites with different fiber volume fractions and the stress distribution on the two Mohr circles [296].

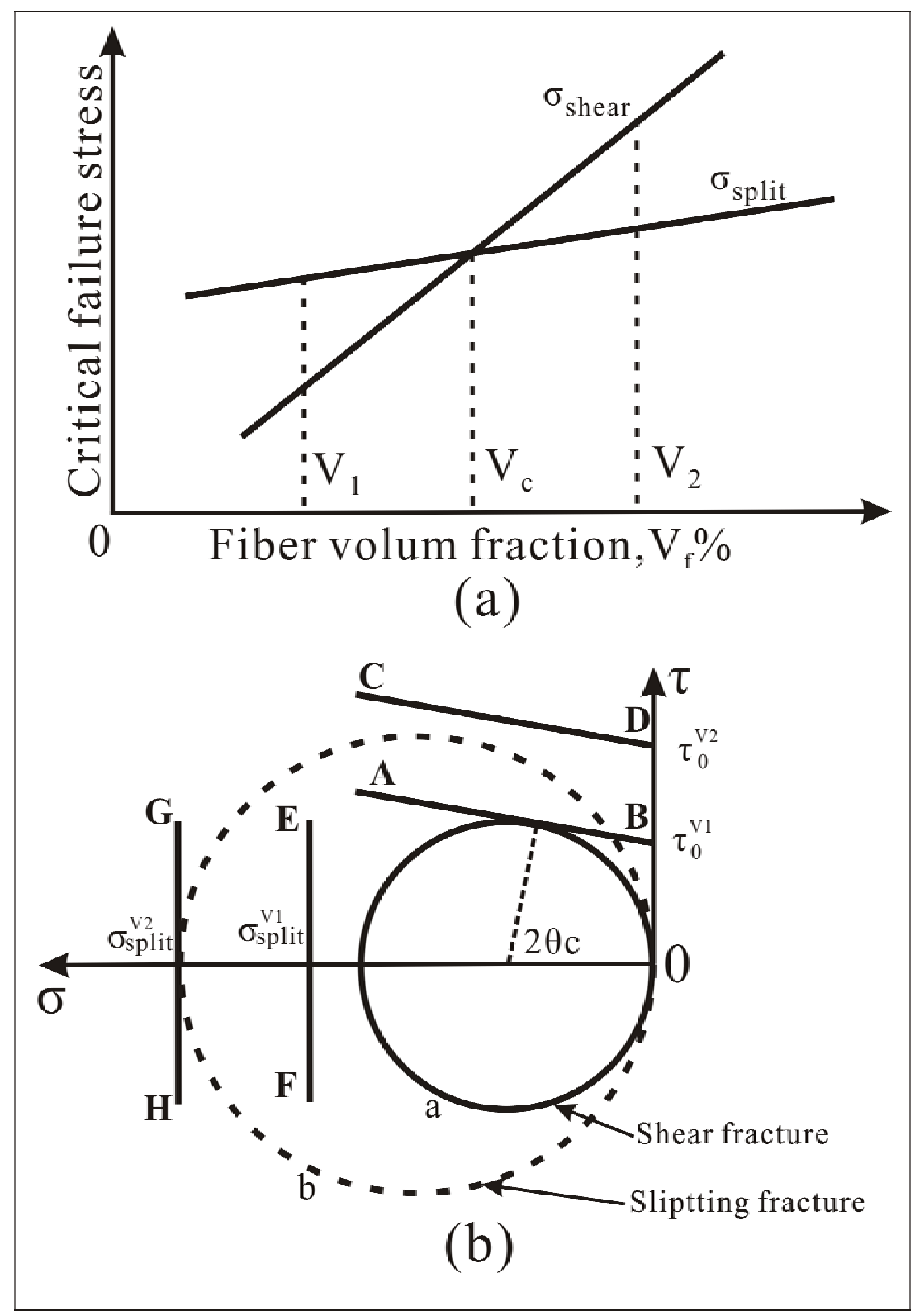


Figure 34 Specific strength and plasticity of a series of monolithic BMGs and ex-situ particle-reinforced MGMCs [48].

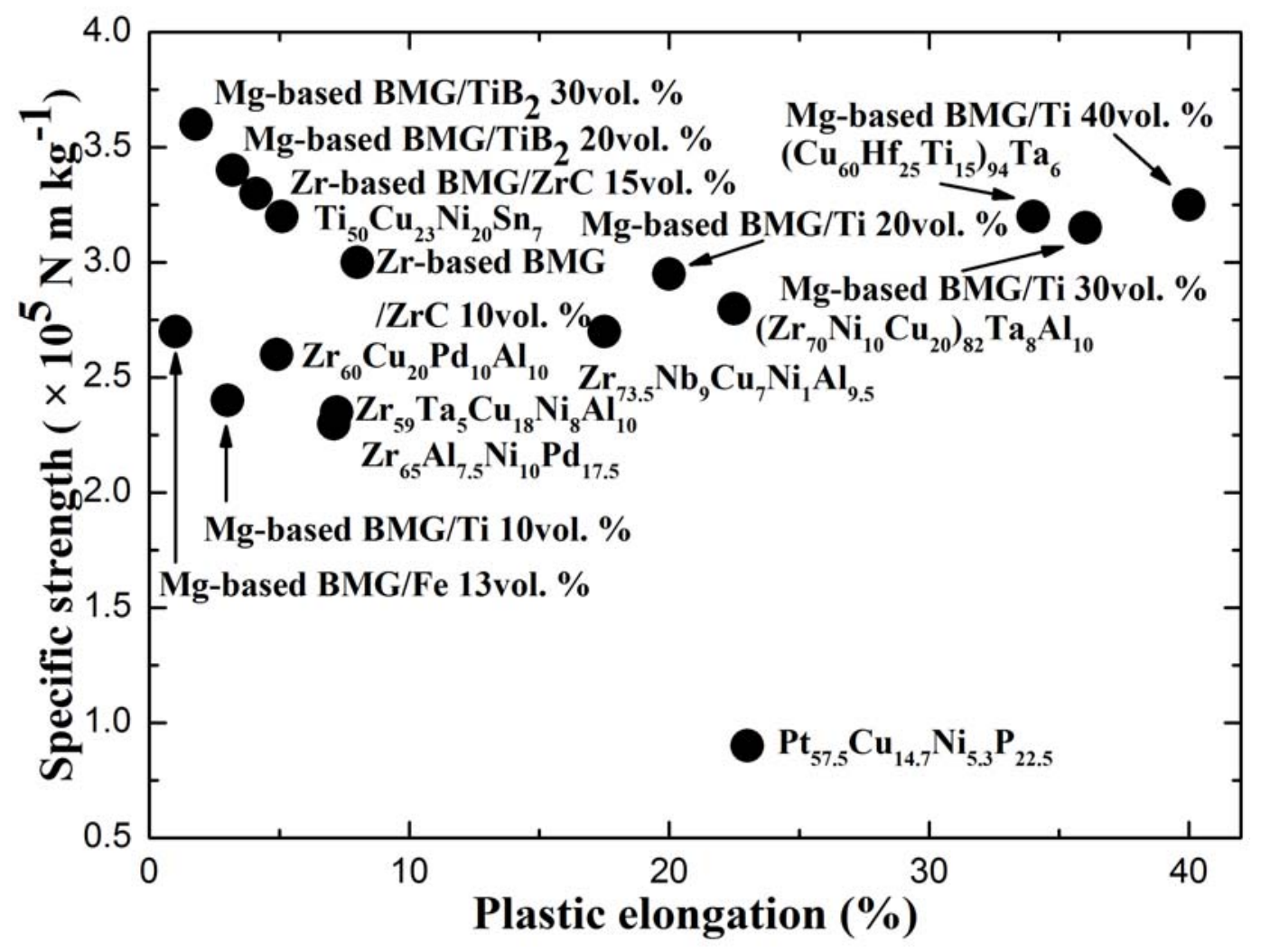


Figure 35 The compressive engineering stress-strain curves of the

$\mathrm{Zr}_{58.5} \mathrm{Ti}_{14.3} \mathrm{Nb}_{5.2} \mathrm{Cu}_{6.1} \mathrm{Ni}_{4.9} \mathrm{Be}_{11.0}$ composite, and its corresponding dendrite and glass matrix.

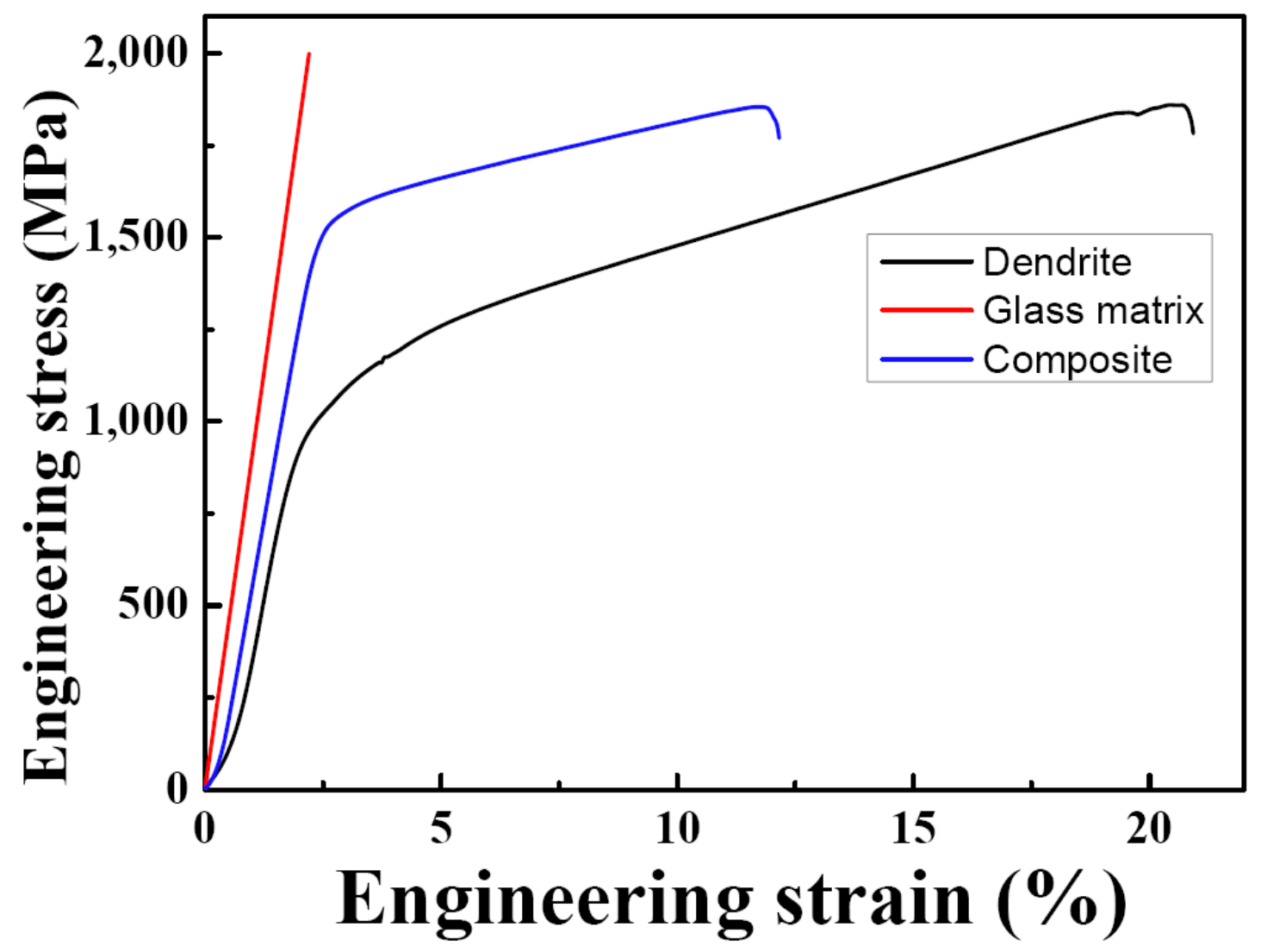


Figure 36 The compressive stress-strain curves at four different test temperatures (77, 294, 373, and $473 \mathrm{~K})$ [59].

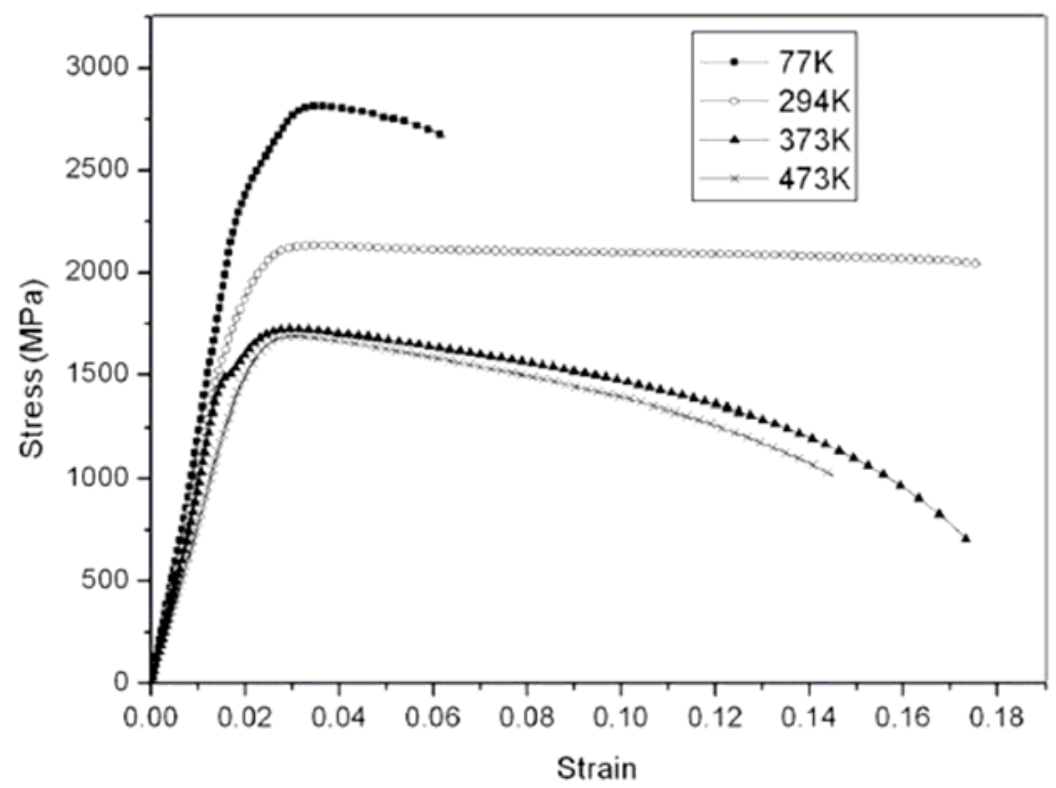


Figure 37 Comparison of the stress-strain responses at various testing temperatures: (a) at room temperature $(300 \mathrm{~K}),(\mathrm{b})$ at high temperature but below $T_{\mathrm{g}}(550 \mathrm{~K}),(\mathrm{c})$ at $T_{\mathrm{g}}$ $(600 \mathrm{~K})$, and $(\mathrm{d})$ above $T_{\mathrm{g}}(650 \mathrm{~K})$. Note that the plots obtained at the strain rate of $10^{-2}$ $\mathrm{s}^{-1}$ were not shown for the sake of clarity [302].
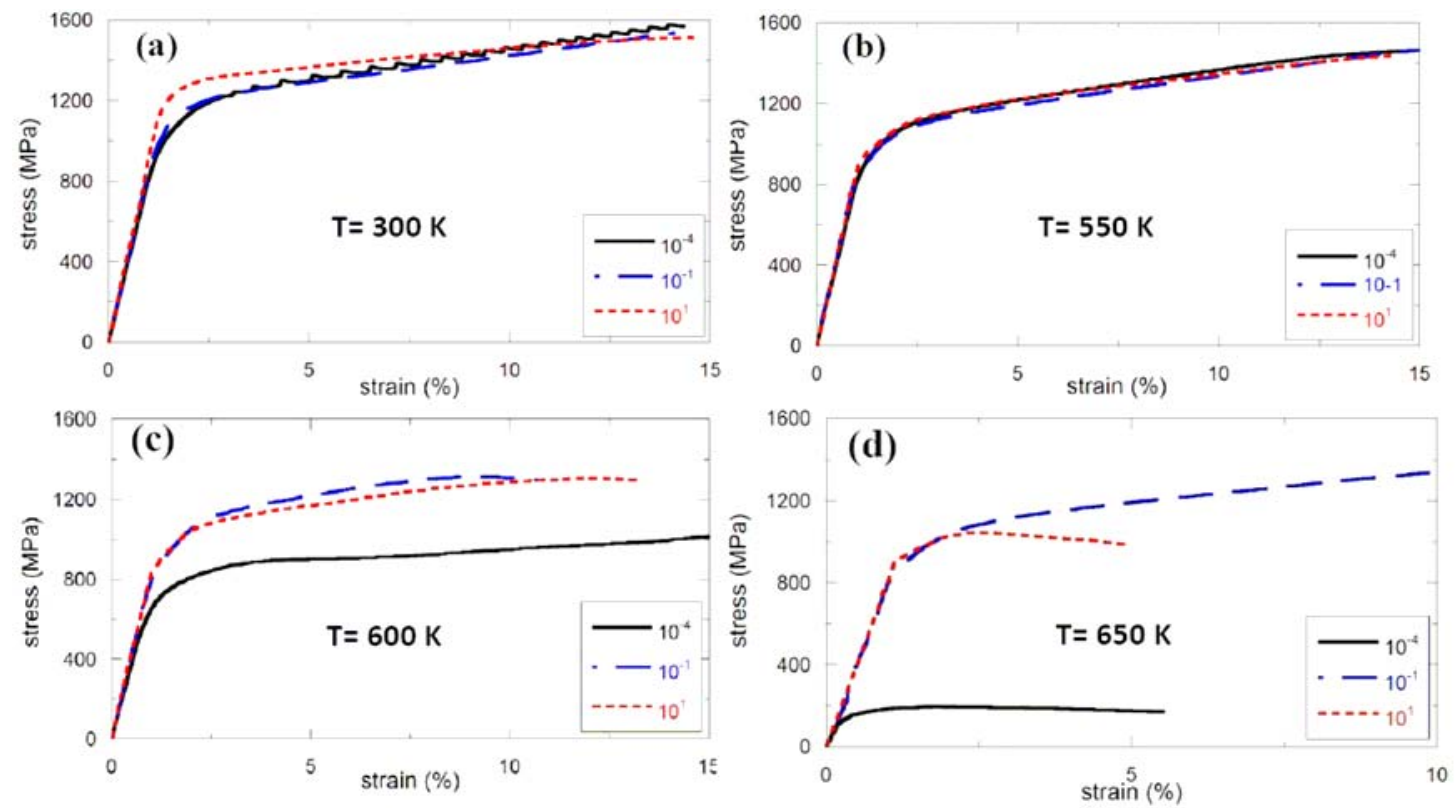
Figure 38 The true stress-strain curves measured at 77 and $298 \mathrm{~K}$ (a) as well as in the supercooled liquid region, $693 \mathrm{~K}$ for 70 -vol. \%-W-containing-Vit106 composites with the SEM image and (b) for 5-vol. \%-Ta-containing Zr-based composites with the backscattering SEM image [307].

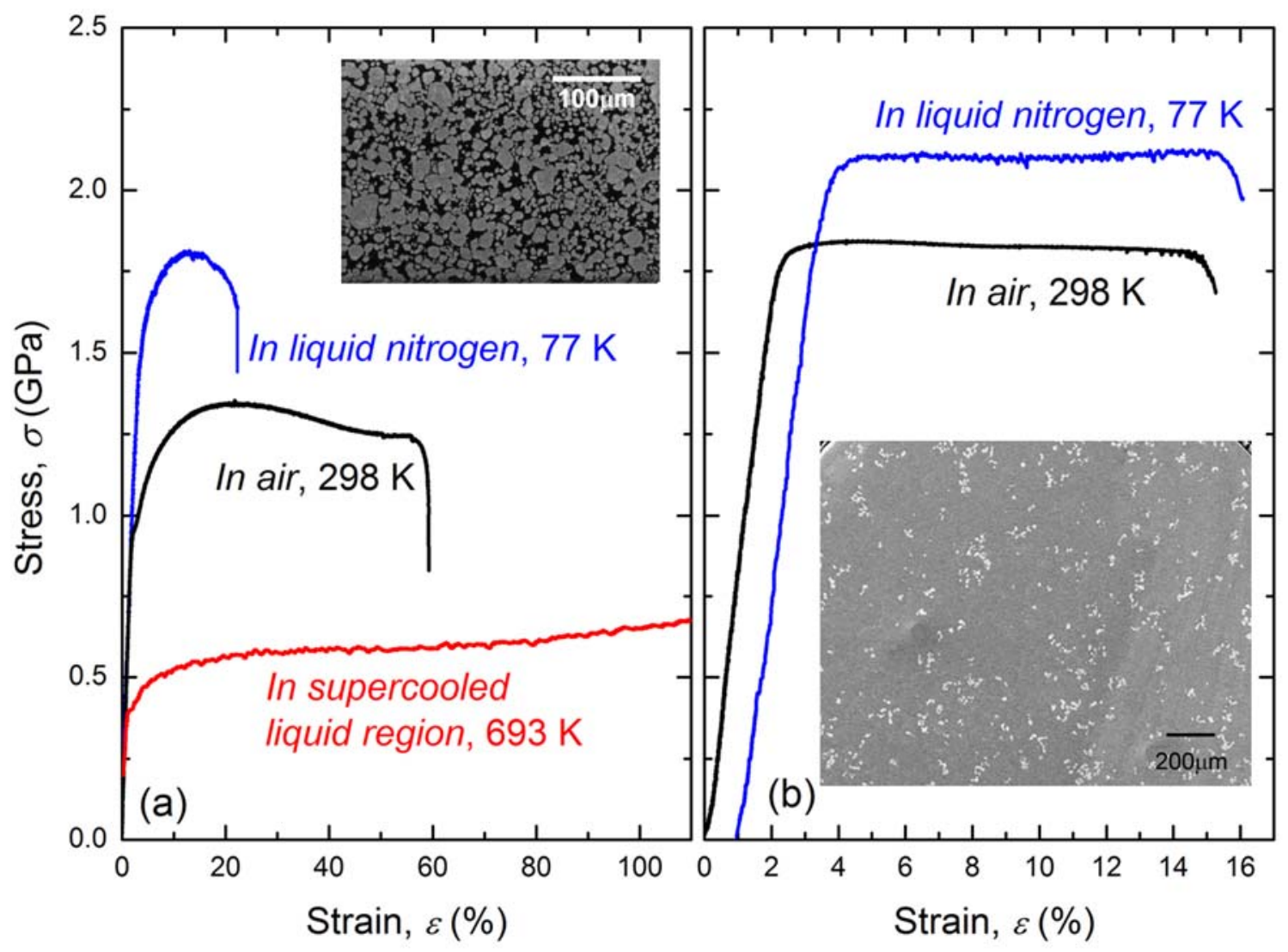


Figure 39 Integrated tensile properties of strength and ductility for in-situ MGMCs.

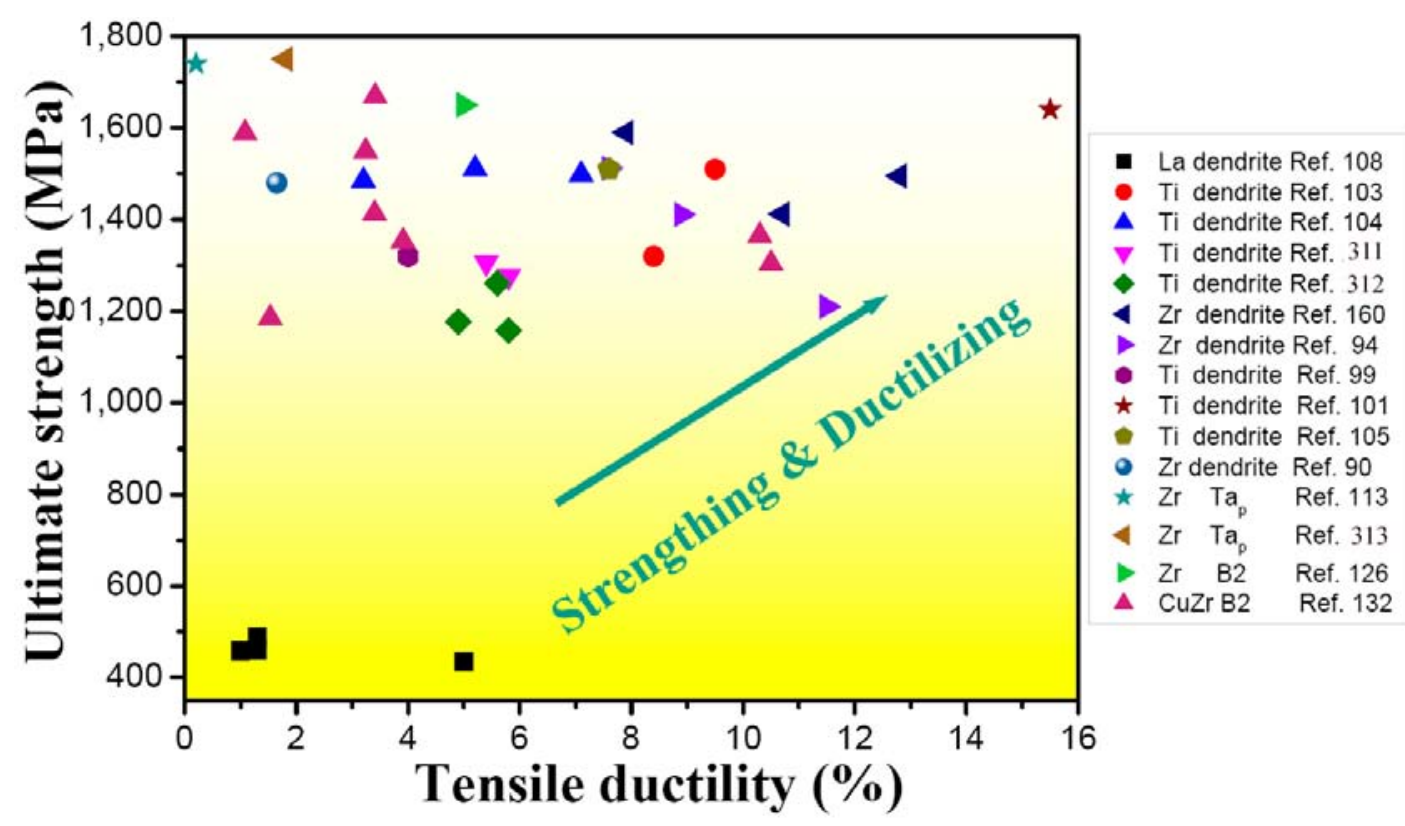


Figure 40 (a) the tensile true stress-strain curves of ex-situ porous tungsten / Zr-based metallic-glass-interpenetrating composites, the inset in (a) showing the microstructure [317]; (b) the stress-displacement curves of in-situ dendrite / Ti-based MGMCs at 613 $\mathrm{K}$ with a volume fraction of dendrites of $43 \%$, the inset indicating pictures of the microstructure and deformed samples [318]; and (c) the tensile true tress-strain curves at $693 \mathrm{~K}$ for the in-situ Ta-particle-reinforced MGMCs with a composition of $\mathrm{Zr}_{55.9} \mathrm{Cu}_{18.6} \mathrm{Ni}_{10} \mathrm{Al}_{7.5} \mathrm{Ta}_{8}$, the inset giving the picture of the super elongation and microstructures [319].

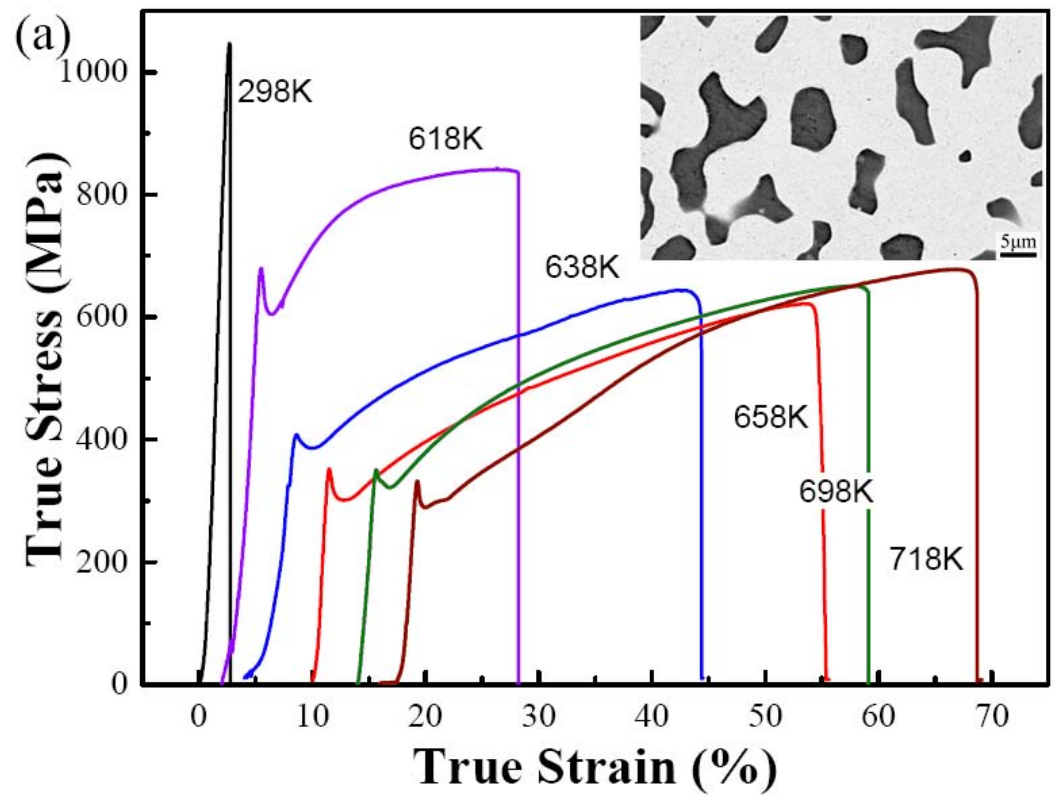



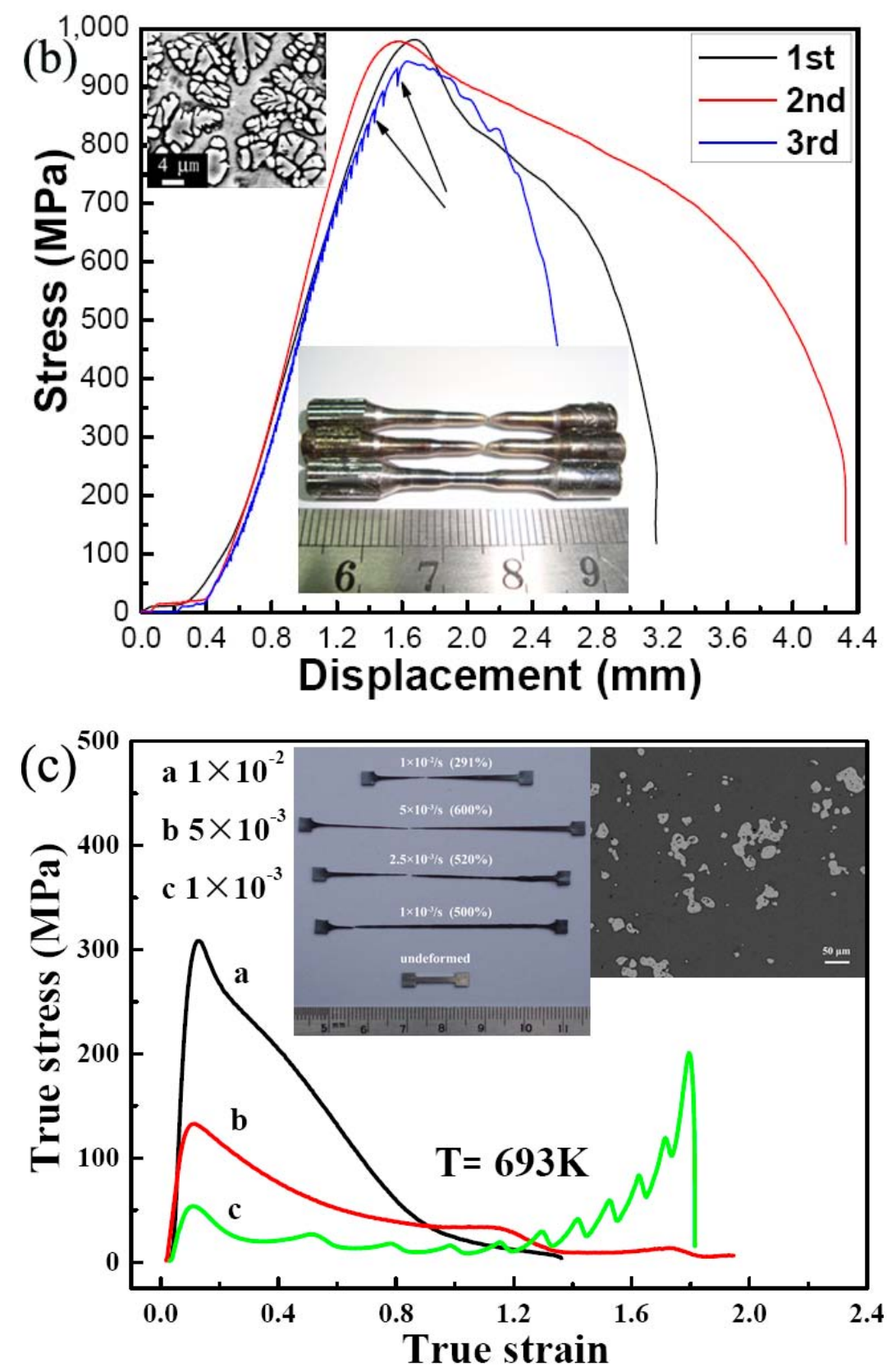
Figure 41 The tensile stress-strain curve of in-situ dendrite MGMCs at $77 \mathrm{~K}$ (a); the pictures of samples before and after deformation (b); and the diffraction pattern after deformation (c) [322].

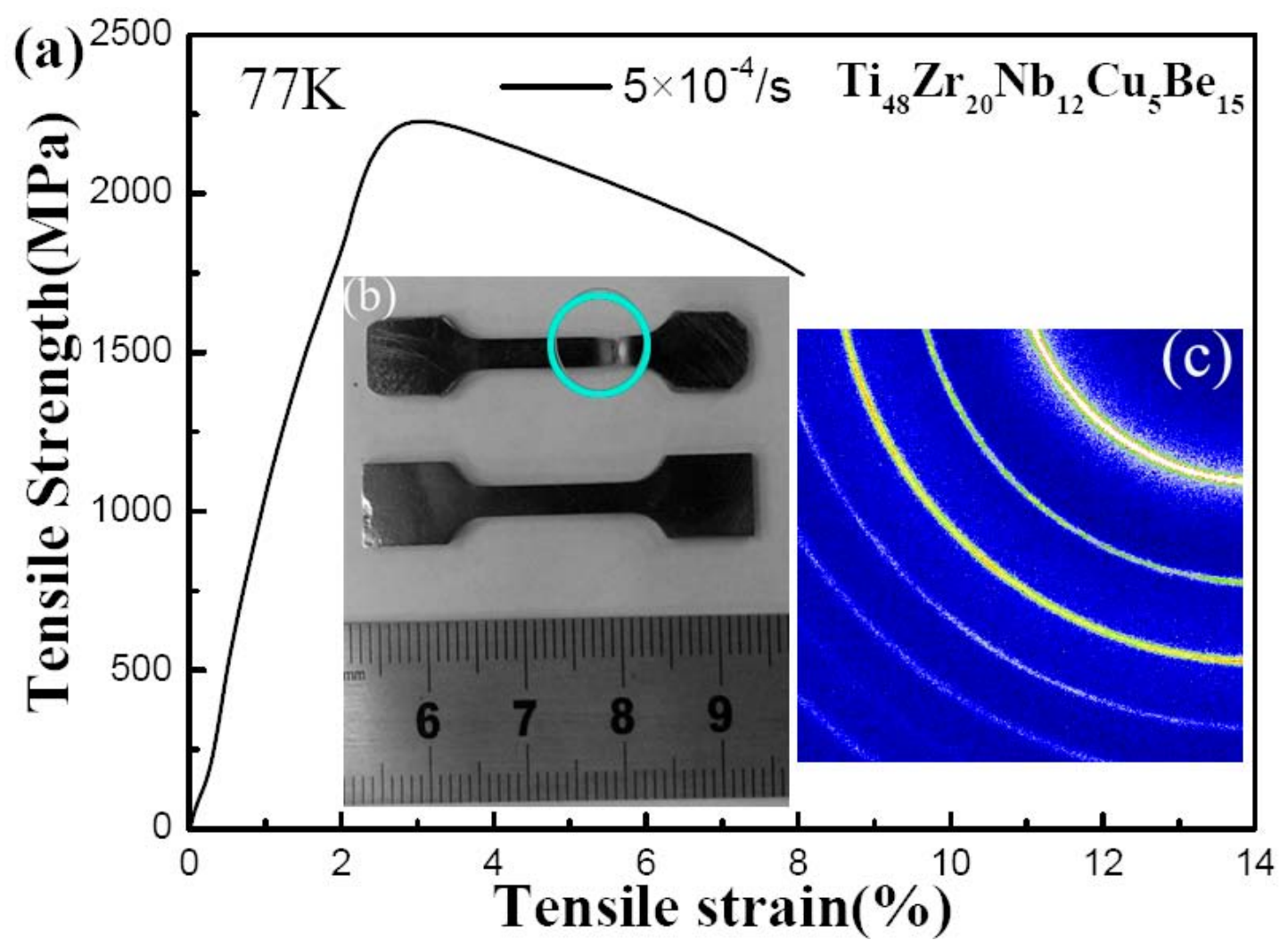


Figure 42 (a) schematic of the composite structure consisting of second-phase nanocrystals. Colors are assigned to identify structural features: blue for the glass matrix, grey for the secondary crystalline phase, and green for the glass-crystal interfaces and the free surfaces, (b) three composites embedded with different shapes of nanocrystals, i.e., $b / a=6.5,5.7$, and 3.7. The corresponding volume fractions of the crystalline phase are $16.7 \%, 14.5 \%$, and $9.4 \%$, respectively, (c) atomistic configurations of the structural evolution in 'Composite II' upon deformation, (d) and (e) typical deformation patterns illustrating structural evolution in the composites and monolithic BMG, respectively, (f) atomistic configuration of 'Composite Inclined' at a strain of 15\%, (g) and (h) atomic configurations of 'Composite Random' at various applied strains, and (i) atomic configurations of 'Composite Dendritic' upon deformation [327]. 


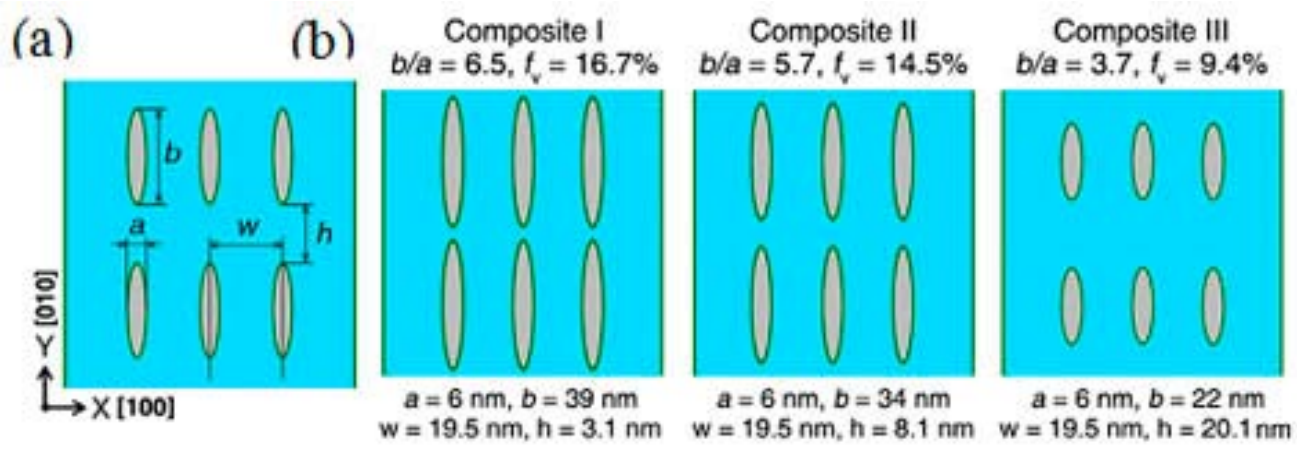

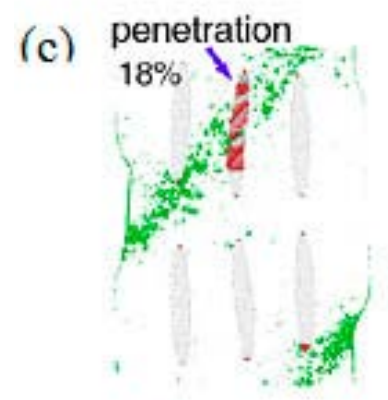

(d)

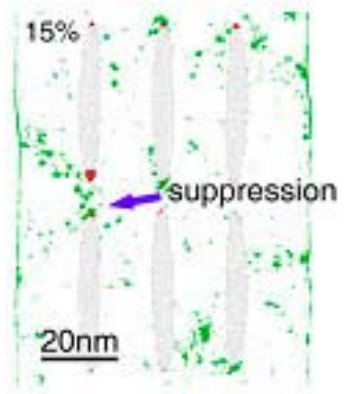

(f)

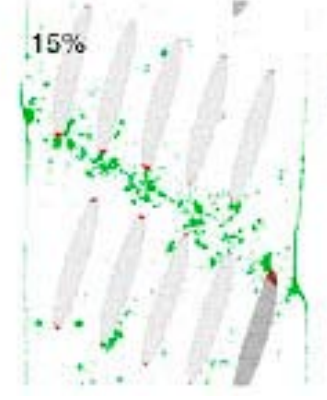

(g)

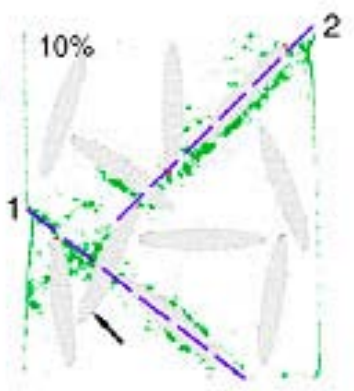

(e)

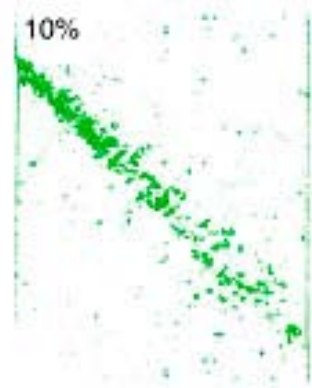

(h) $20 \%$

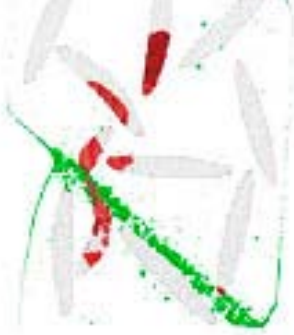


Figure 43 Shear-strain contours of a nominal strain of $11.5 \%$ for (a) Comp1, (b)

Comp2, and (c) Comp3, with circular, large dendrites, and small dendrite crystalline inclusions, respectively. Dark domains represent a glassy phase, while light domains indicate for ductile phases [326].

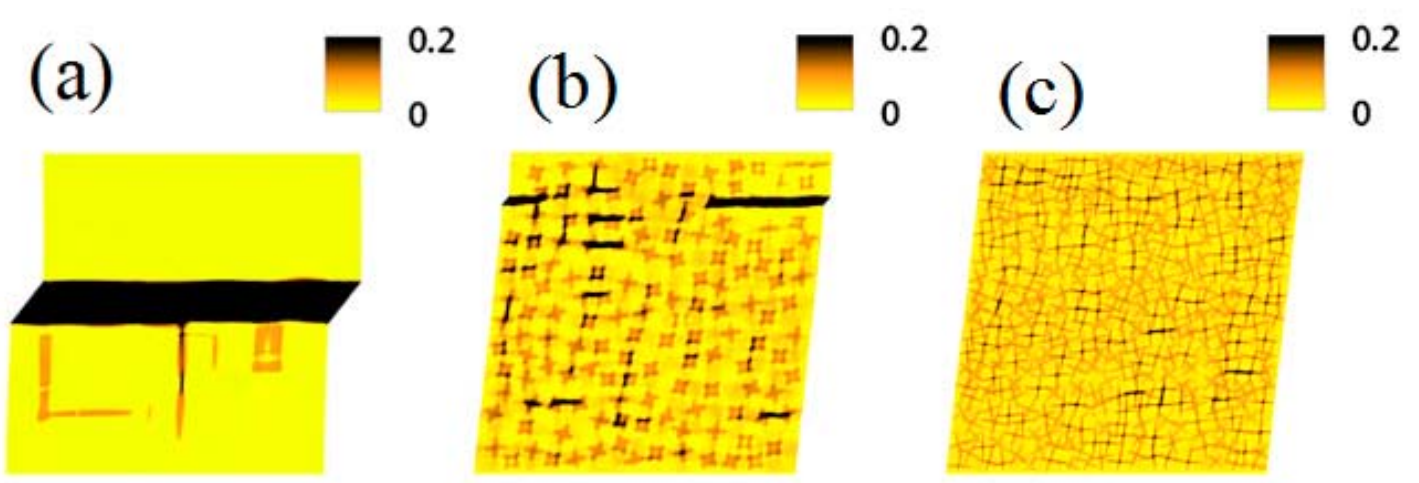


Figure 44 (a) typical stress-strain curves for composites with various volume fractions of second-phase nanocrystals, (b) - (d) Atomic configurations at various applied strains demonstrating the structural evolution in 'Composite VII'. The arrow indicates the activation of lattice sliding at a strain of $13 \%$, (e) and (f) schematics illustrating the transition in the governing deformation mechanism of the glass subdomains as the volume fraction of the crystalline second phase increases [327].

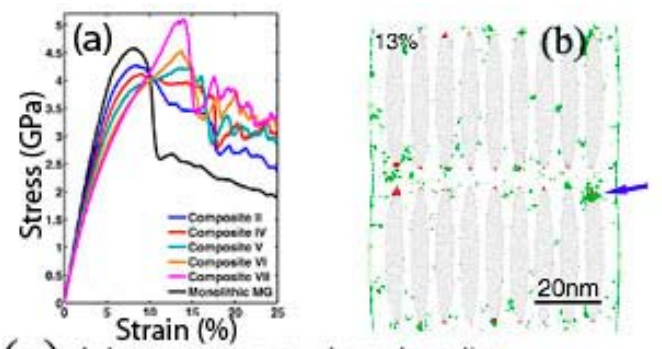

(e) Inhomogeneous shear banding
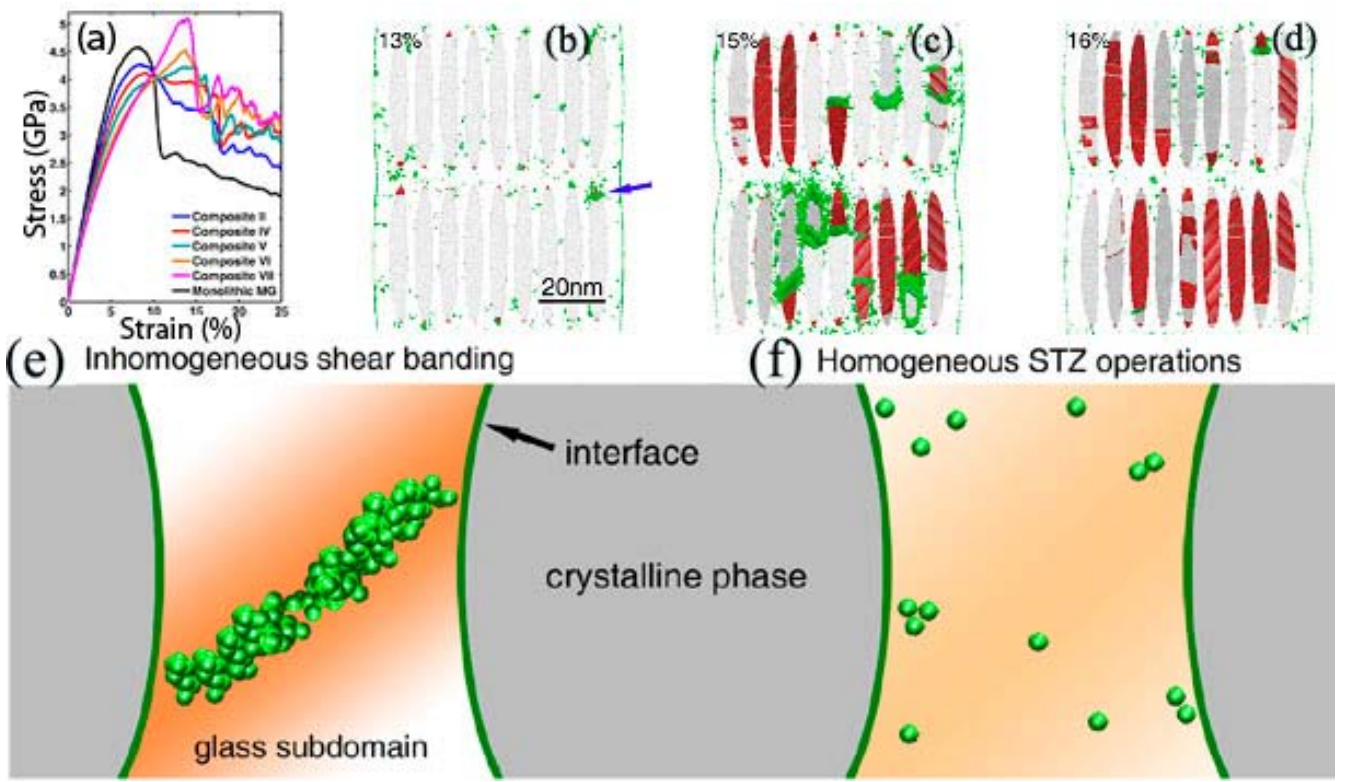
Figure 45 Different deformation modes for shear banding and crack propagation in MGMCs: (a) and (b) shear-banding-induced cracking inside the reinforcement after the shear band interacts with reinforcements. $\mathrm{g}=0, \mathrm{w}=0 ; K_{\mathrm{I}}=24.2 \mathrm{MPa} \sqrt{\mathrm{m}}(\mathrm{a})$, and 27.5 MPa $\sqrt{\mathrm{m}}(\mathrm{b})$; (c) propagation of shear bands along the reinforcement surface with a compressive residual stress $\mathrm{g}=-435 \mathrm{MPa} ; \mathrm{w}=0$; (d) shear-band-induced cracking inside the reinforcement with a tensile residual stress, $g=175 \mathrm{MPa}$ at its surface; $\mathrm{w}=$ 0 ; (e) branching of shear bands along the reinforcement surface; $\mathrm{w}=0.8 ; \mathrm{g}=0$; and (f) shear banding at opposite sides of the reinforcement surface; and $\mathrm{w}=1$; $g=0 . \mathrm{K}_{\mathrm{I}}=$ 27.5 $\mathrm{MPa} \sqrt{\mathrm{m}}$ in (c)-(f) [325].
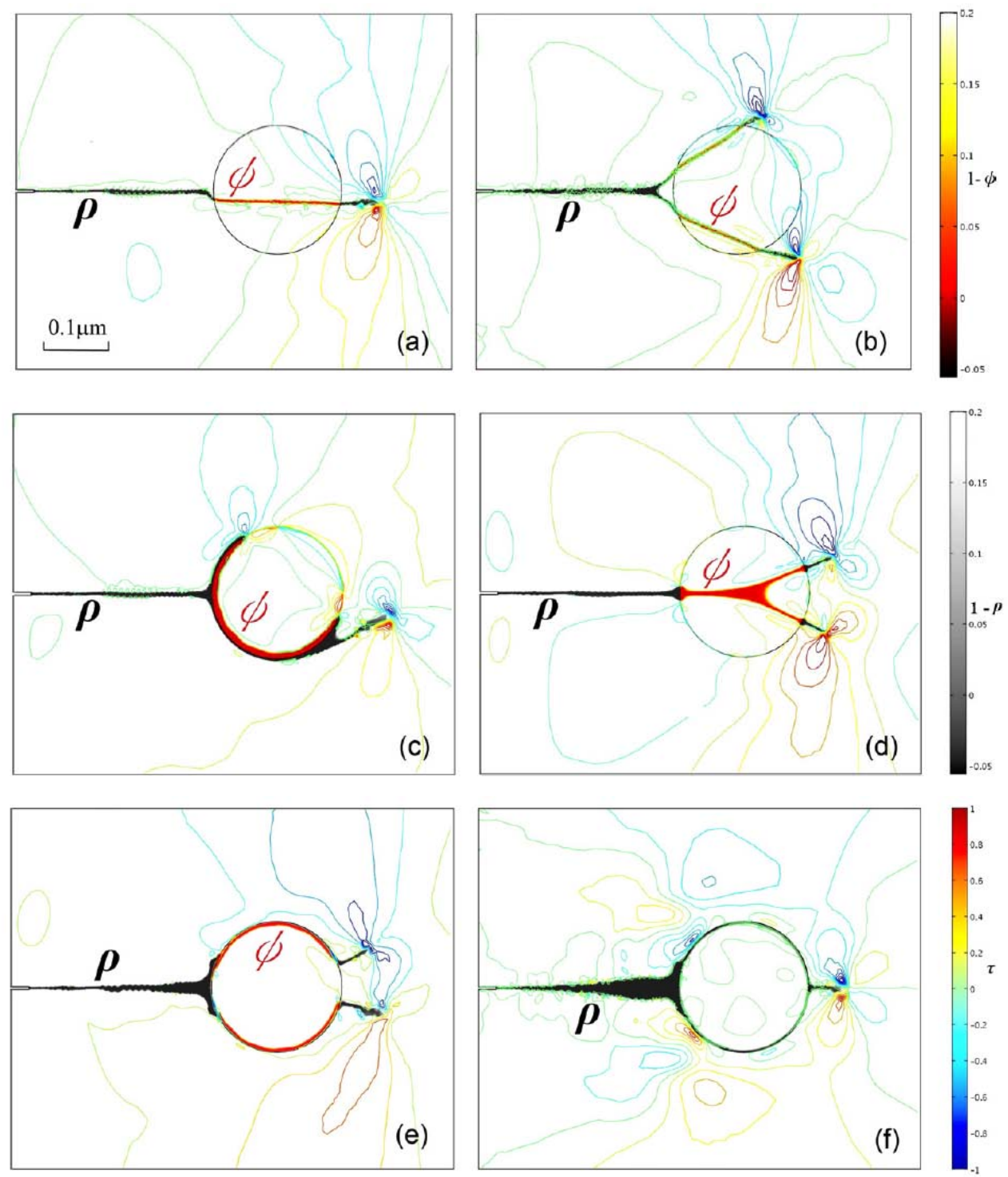
Figure 46 Low-magnification optical photographs of the dynamically-deformed compressive specimen for the (a) stainless steel (S), and (b) Tantalum (T) fiber reinforced specimens [65].

(a)

\section{S Specimen}

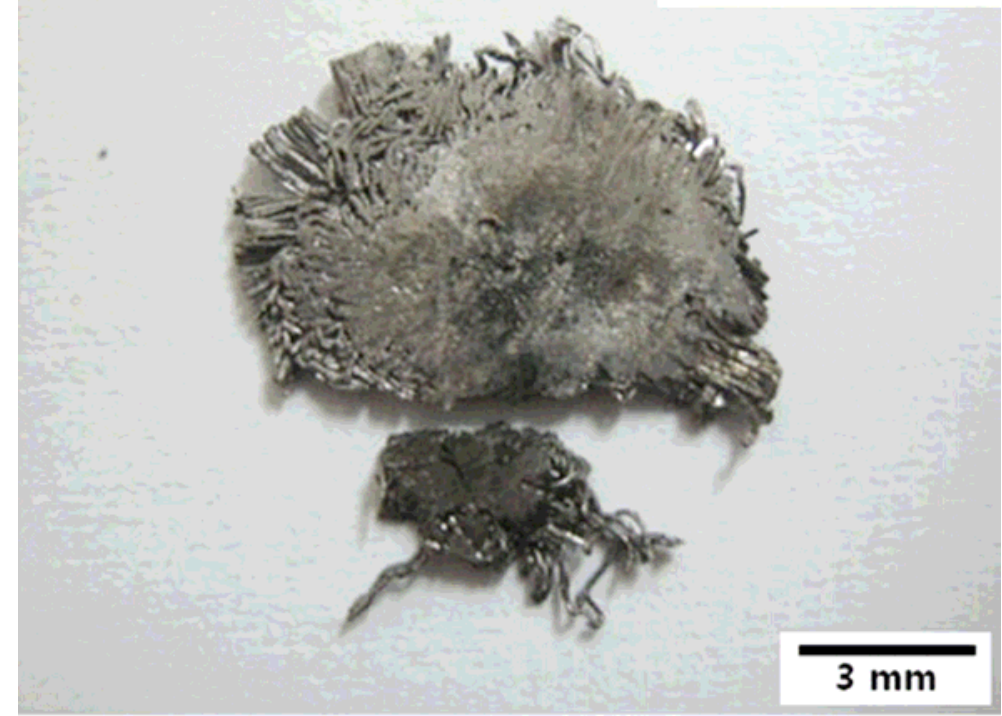

(b)

T Specimen

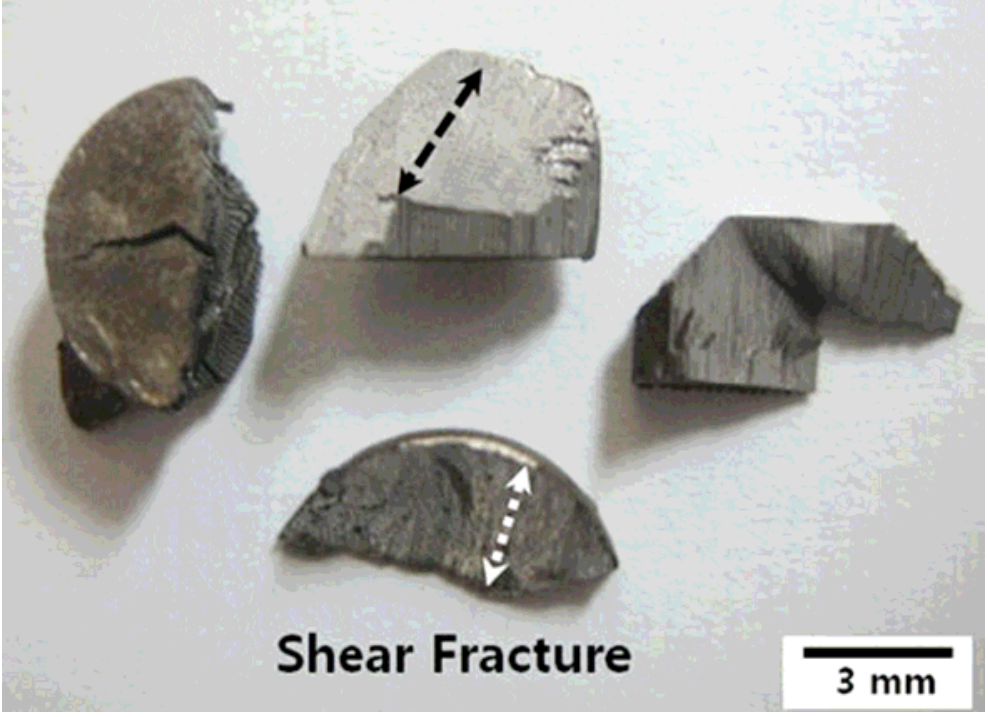


Figure 47 The engineering stress-strain curve of the present in-situ metallic glass matrix composites upon quasi-static compression (a); the fractograph of the deformed samples upon quasi-static loading (b); the magnified deformation region near the crack (c); the engineering stress-strain curves upon high-speed dynamic loading (d); and the SEM image of the magnified lateral surface (e) [348].
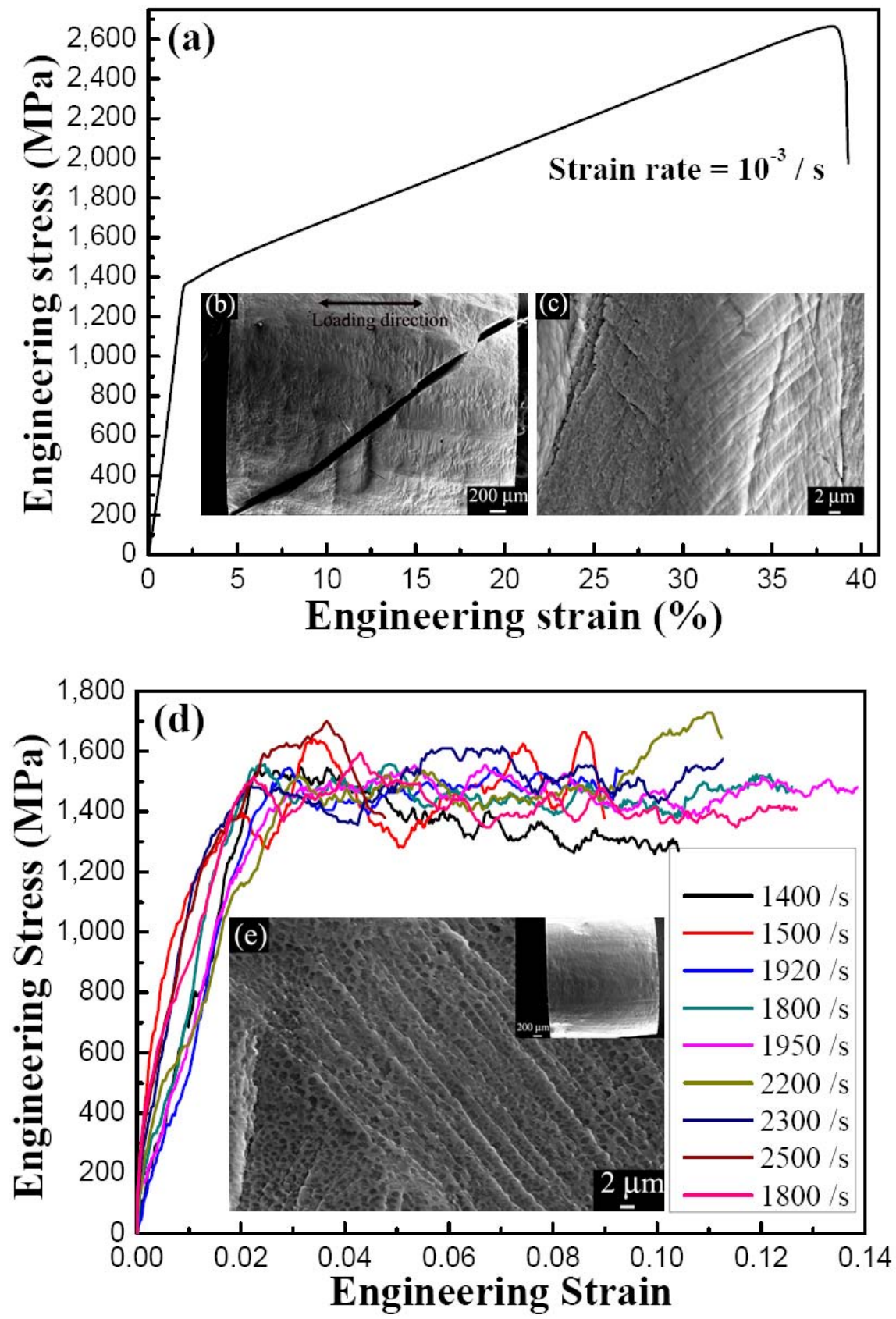
Figure 48 The stress-strain curves of $\mathrm{Cu}_{46} \mathrm{Zr}_{46} \mathrm{Al}_{8}$ MGMCs upon dynamic loading [322].

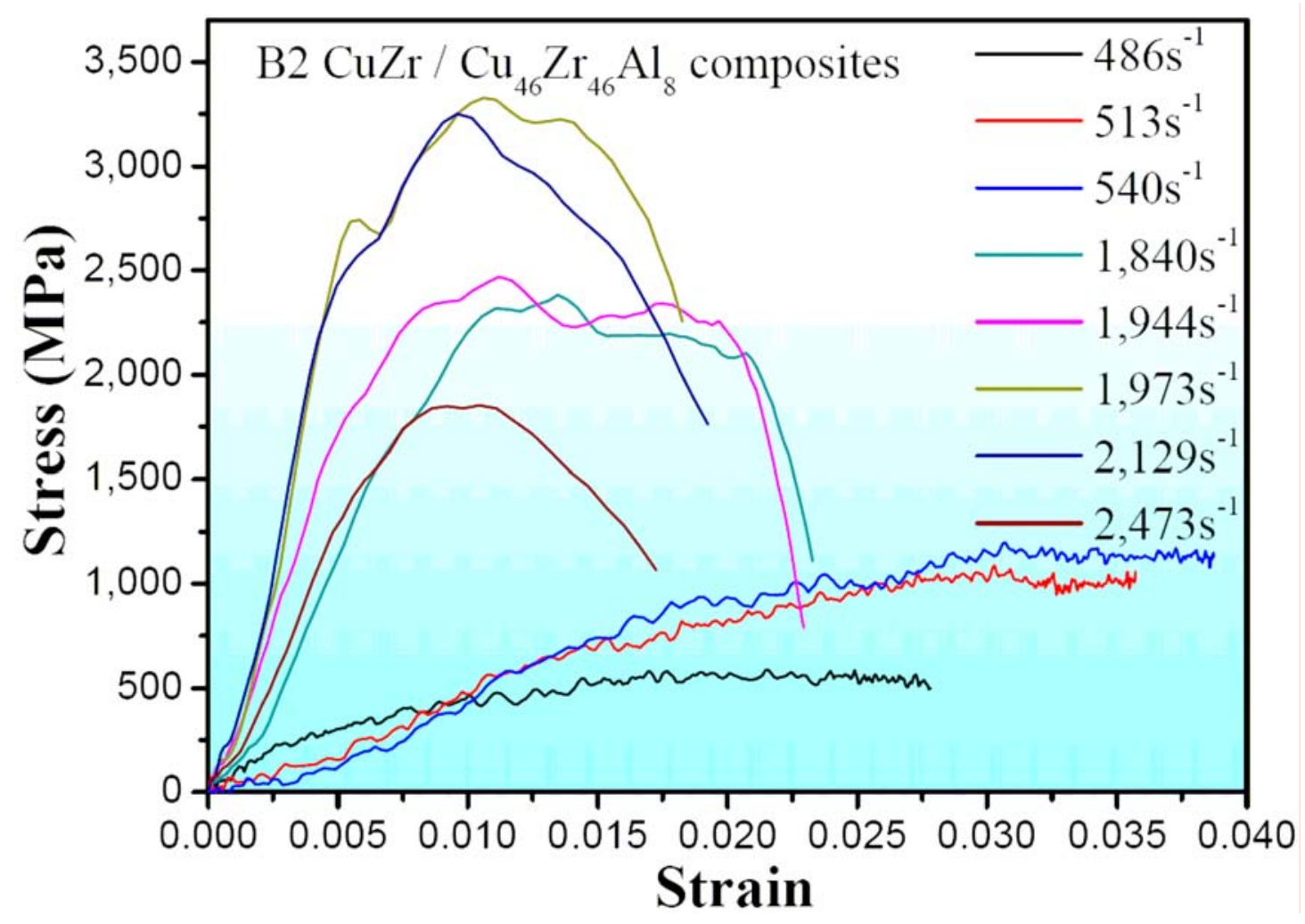


Figure 49 The stress-strain curve of monolithic BMGs upon dynamic tension shown in (a) [322]; and typical true stress strain curves of ex-situ porous W / MGMCs under tension at different strain rates shown in (b) [353]; the inset in (a) and (b) showing the lateral surface and fracture morphology, respectively.
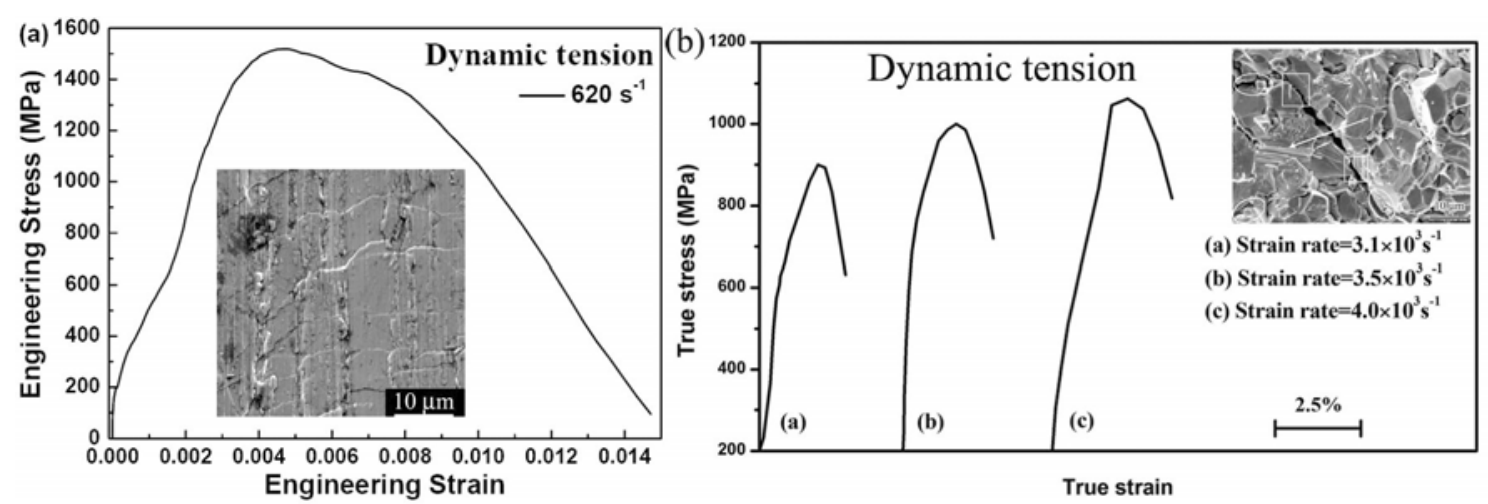
Figure 50 Micrograph of a deformed notch in a glassy $\mathrm{Pd}_{79} \mathrm{Ag}_{3.5} \mathrm{P}_{6} \mathrm{Si}_{9.5} \mathrm{Ge}_{2}$ specimen showing extensive plastic shielding of an initially-sharp crack [371].

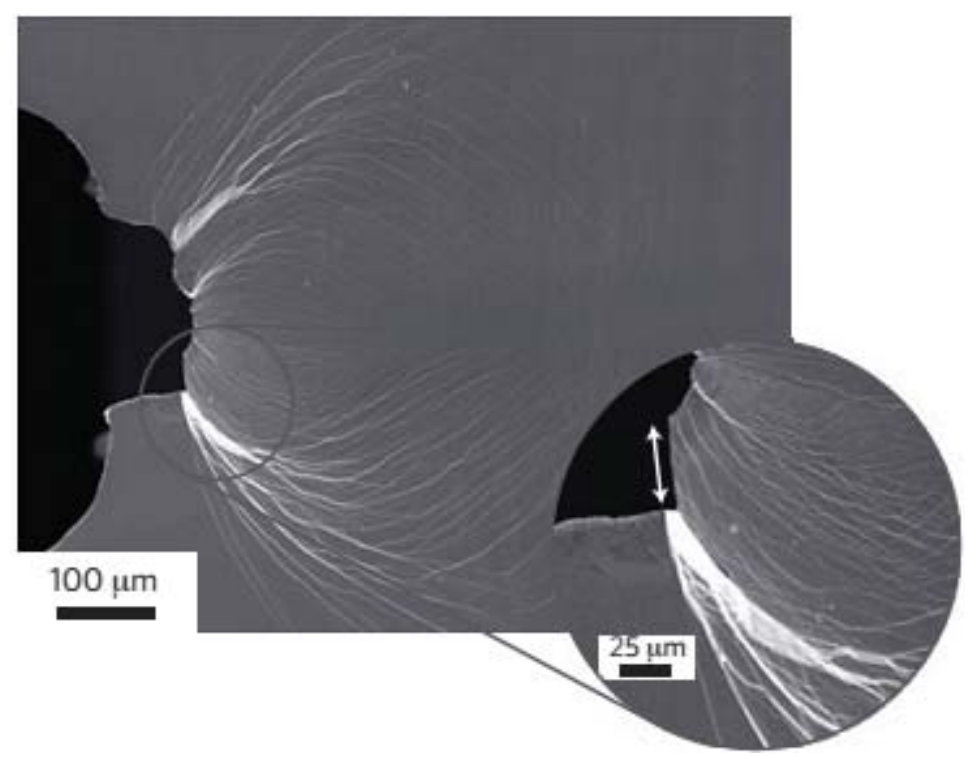


Figure 51 Ranges of fracture toughness versus strength shown for different engineering materials [404].

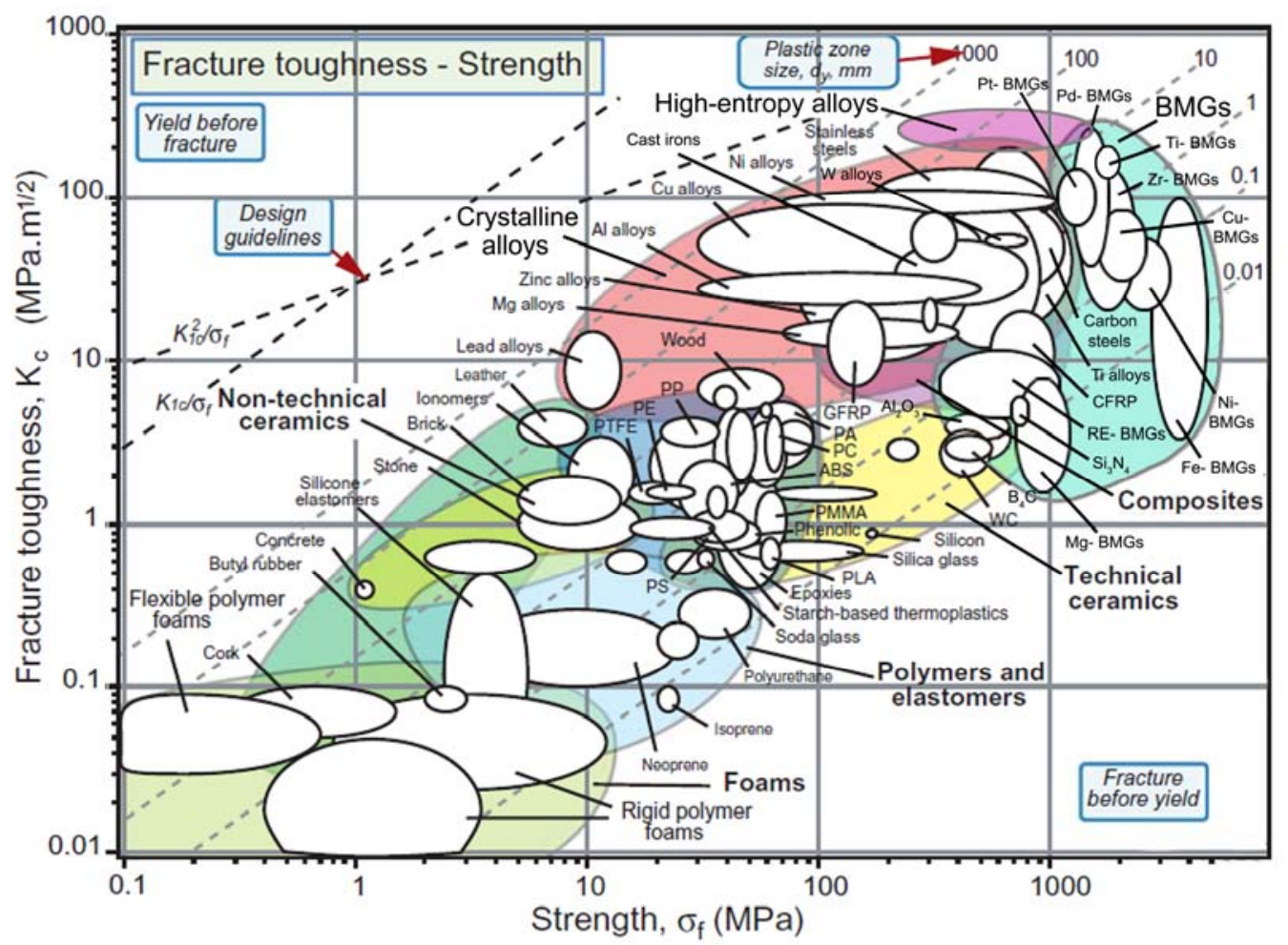


Figure 52 R-curves showing resistance to fracture in terms of the stress intensity, $K_{\mathrm{J}}$ as a function of crack extension, the DH1 and DH3 composite BMG, monolithic Vitreloy 1 BMG, and a 17-7 PH stainless steel [412].

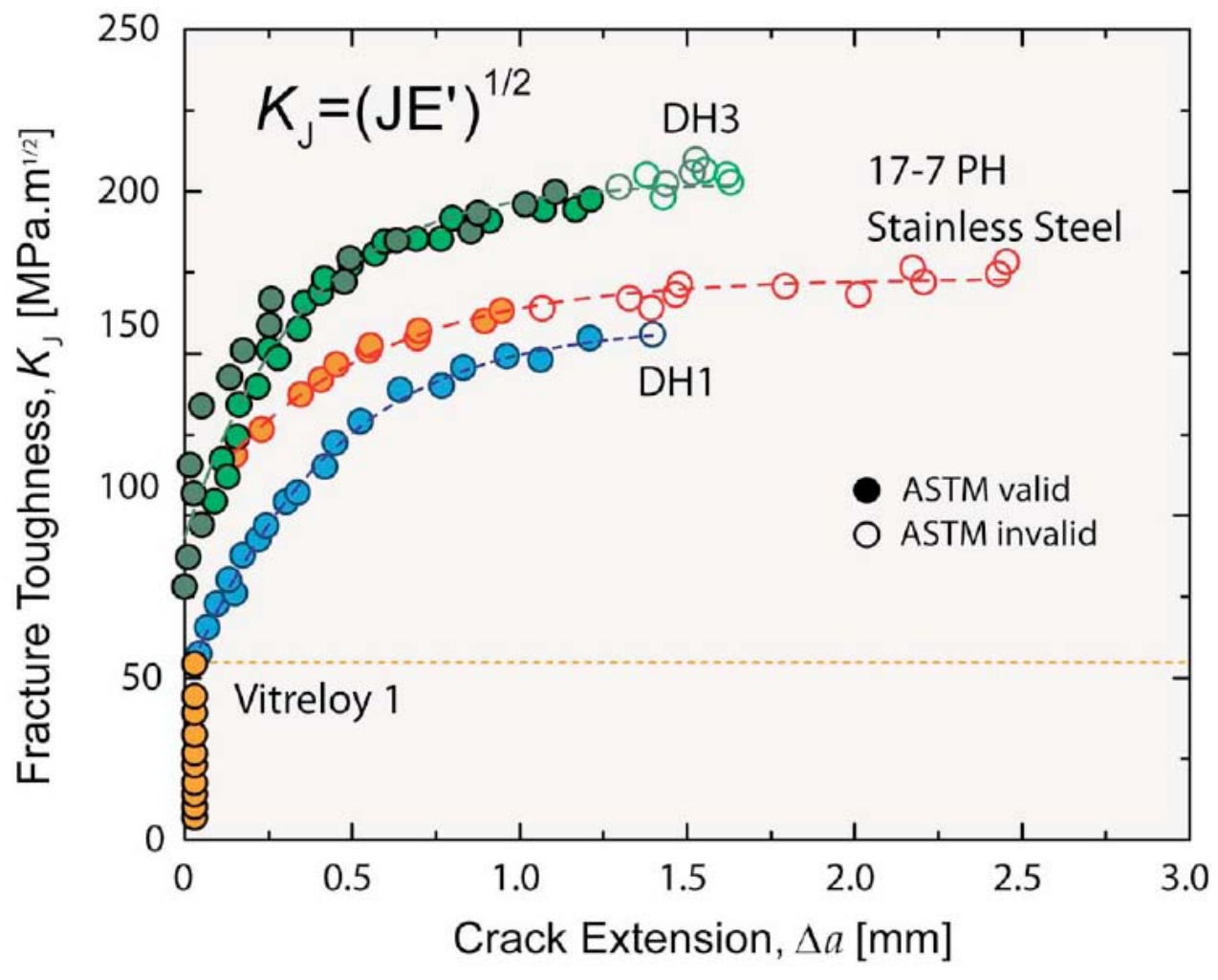


Figure 53 The stress range vs. cycles to failure for three-point-bending (a) and four-point-bending, and (b) fatigue of BMGs [437].
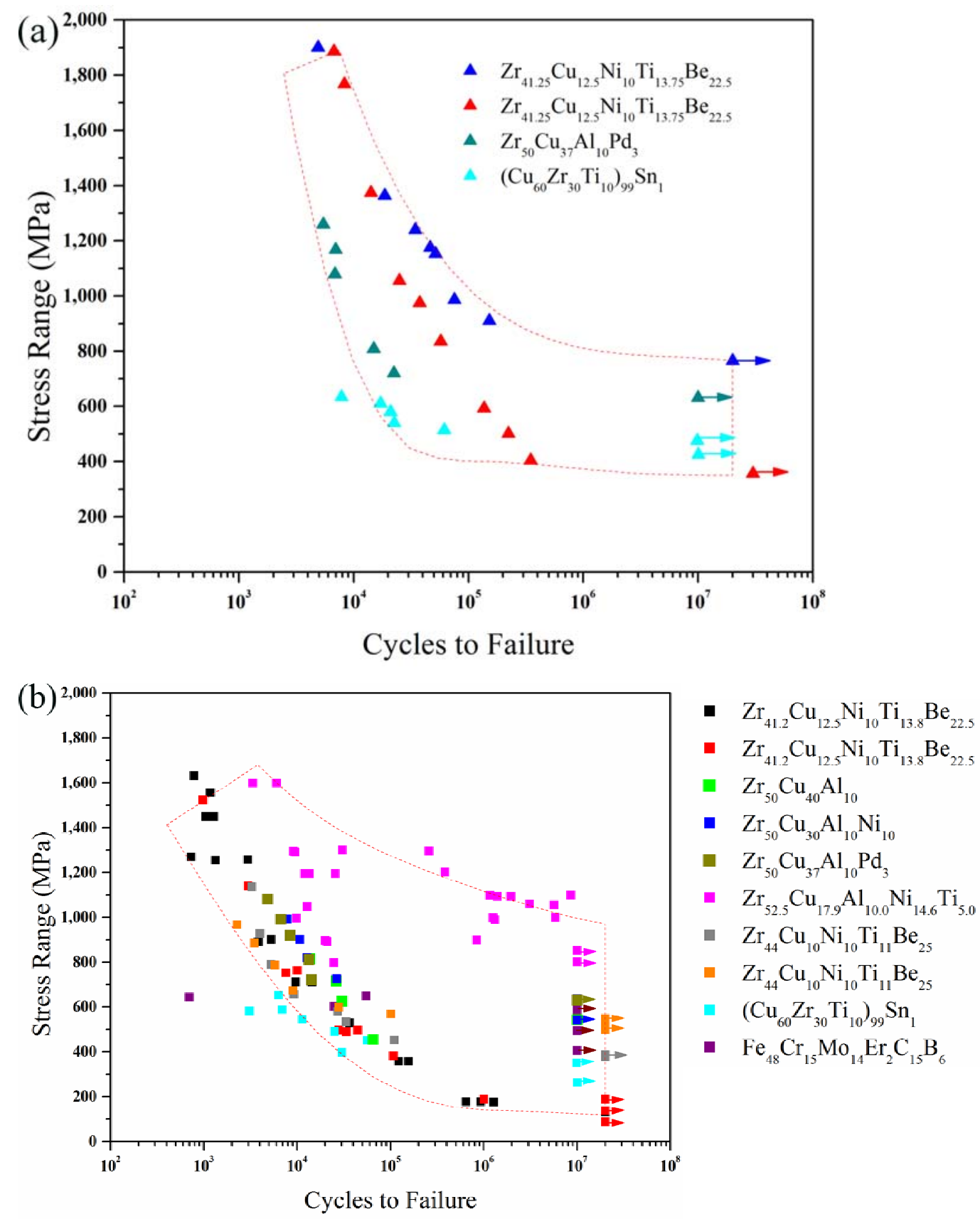
Figure 54 The $\mathrm{S}-\mathrm{N}$ curves of $\mathrm{Zr}_{58.5} \mathrm{Ti}_{14.3} \mathrm{Nb}_{5.2} \mathrm{Cu}_{6.1} \mathrm{Ni}_{4.9} \mathrm{Be}_{11.0}$ MGMCs upon four-point-bending and tension-tension fatigue (a) [442], and the S-N curves of $\mathrm{Zr}_{48} \mathrm{Cu}_{47.5} \mathrm{Co}_{0.5} \mathrm{Al}_{4}$ composites upon compression-compression fatigue (b) (unpublished data).
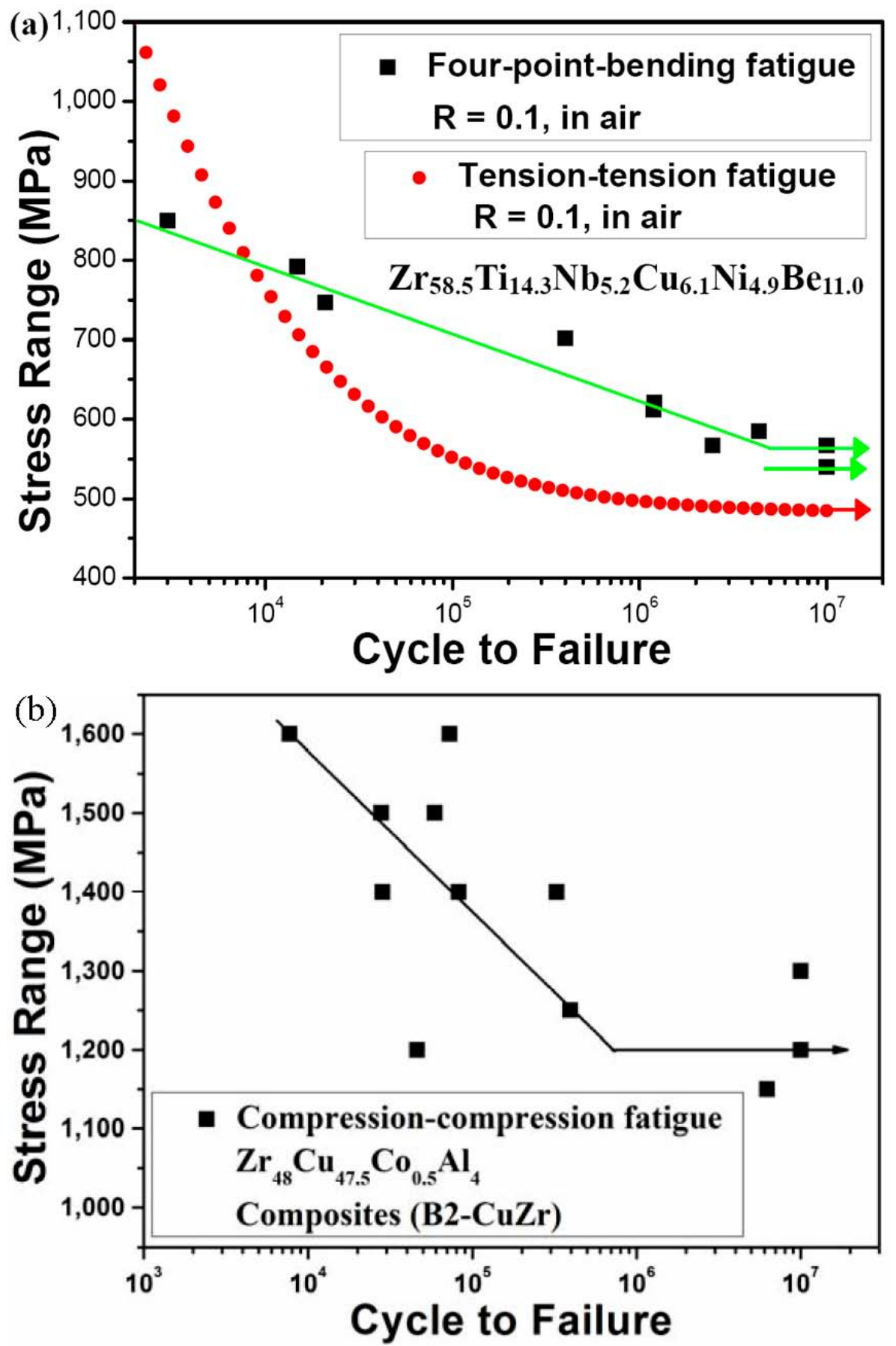
Figure 55 (a) SEM of the lateral surface near the fracture surface of Zr-based MGMCs with a stress range of $792 \mathrm{MPa}$ and cycle number to failure of 14,776; (b) SEM of a fatigue initiation region; (c) SEM of the crack-growth region; (d) SEM of magnified crack-propagation region; (e) SEM of crack-growth region and fast fracture region discerned by a dash line; and (f) SEM of the magnified fast fracture region [442].
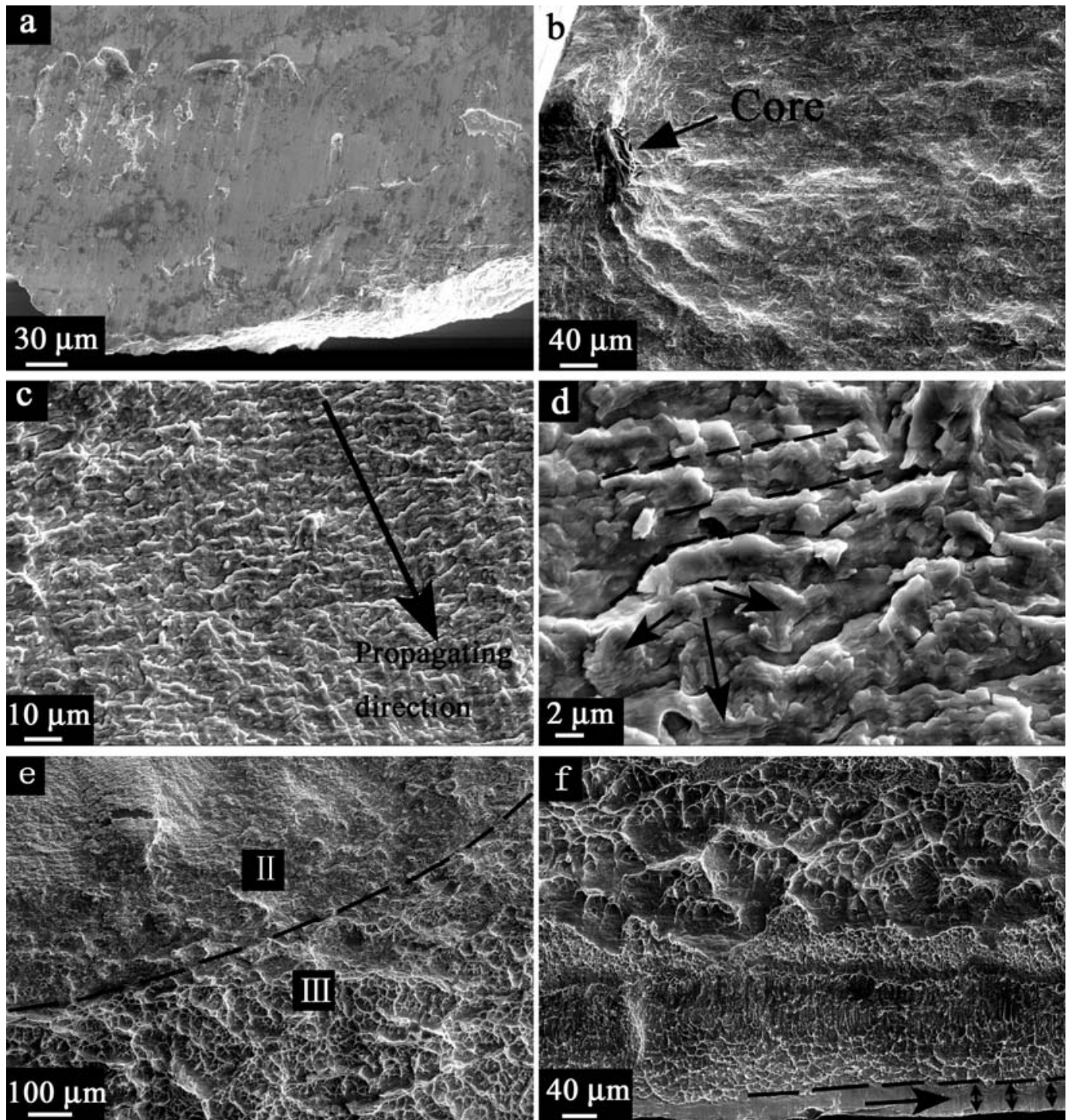
Figure 56 (a) Fatigue fractography of the composites subjected to a stress range of 810 MPa. (b) The enlarged part in stage II near the arresting line in (a) [443].

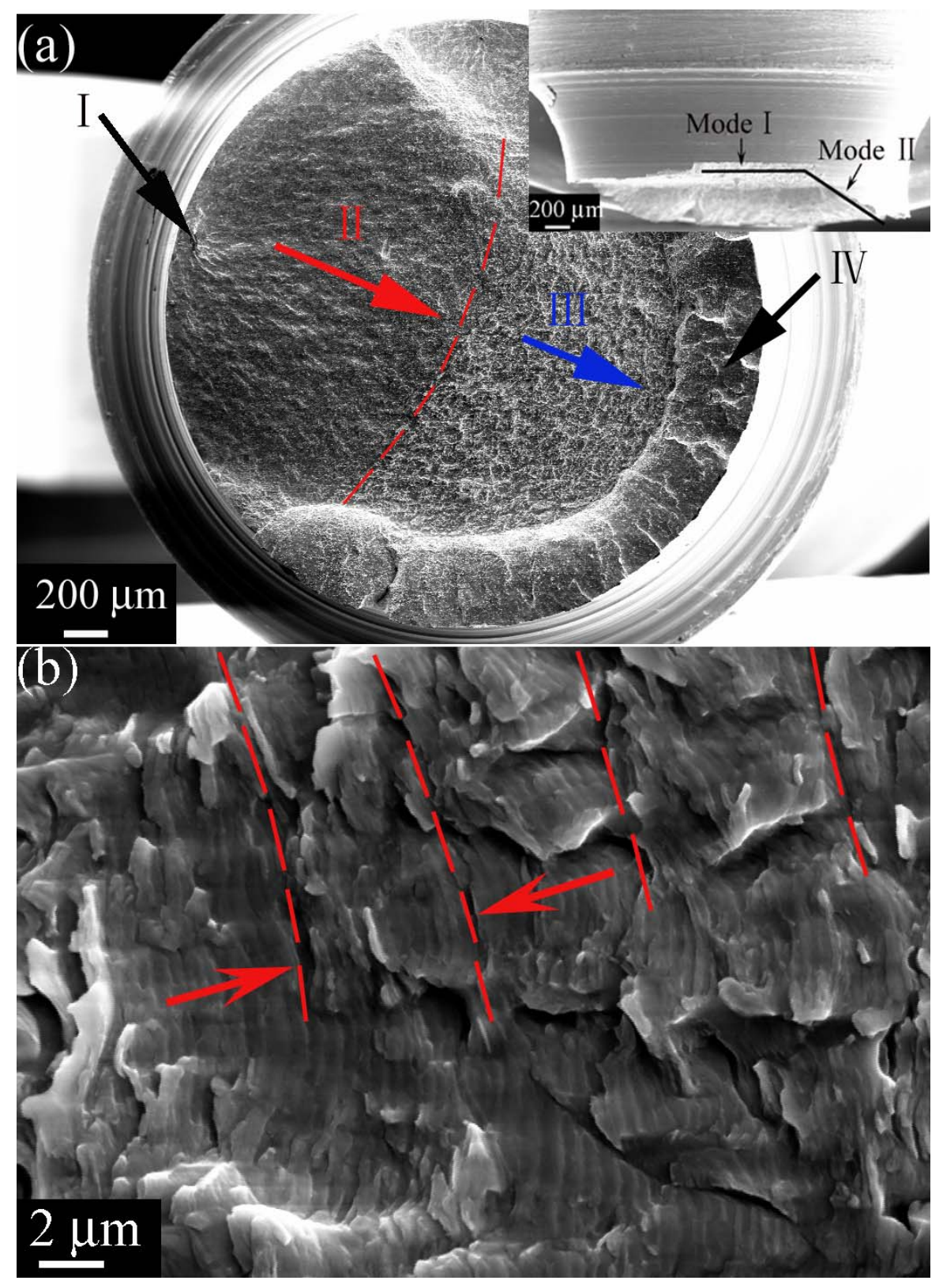


Figure 57 (a) Fatigue-crack-growth rates $(\mathrm{da} / \mathrm{dN})$ vs. the stress-intensity-factor range $(\Delta \mathrm{K})$ for Zr-based BMGs and BMG composites and some crystalline alloys (CT: compact tension, SE: single-edge notch, C: composite, CCT: center-cracked tension, NC: nano-crystalline phase) [436] and (b) The S-N curves of Zr-based BMGs, as well as some typical crystalline alloys [437]. (c) Schematic illustration of the formation of (i) the plastic wake and plastic zone; (ii) the fine striation; and (iii) the coarse striation when a fatigue crack advances [447].
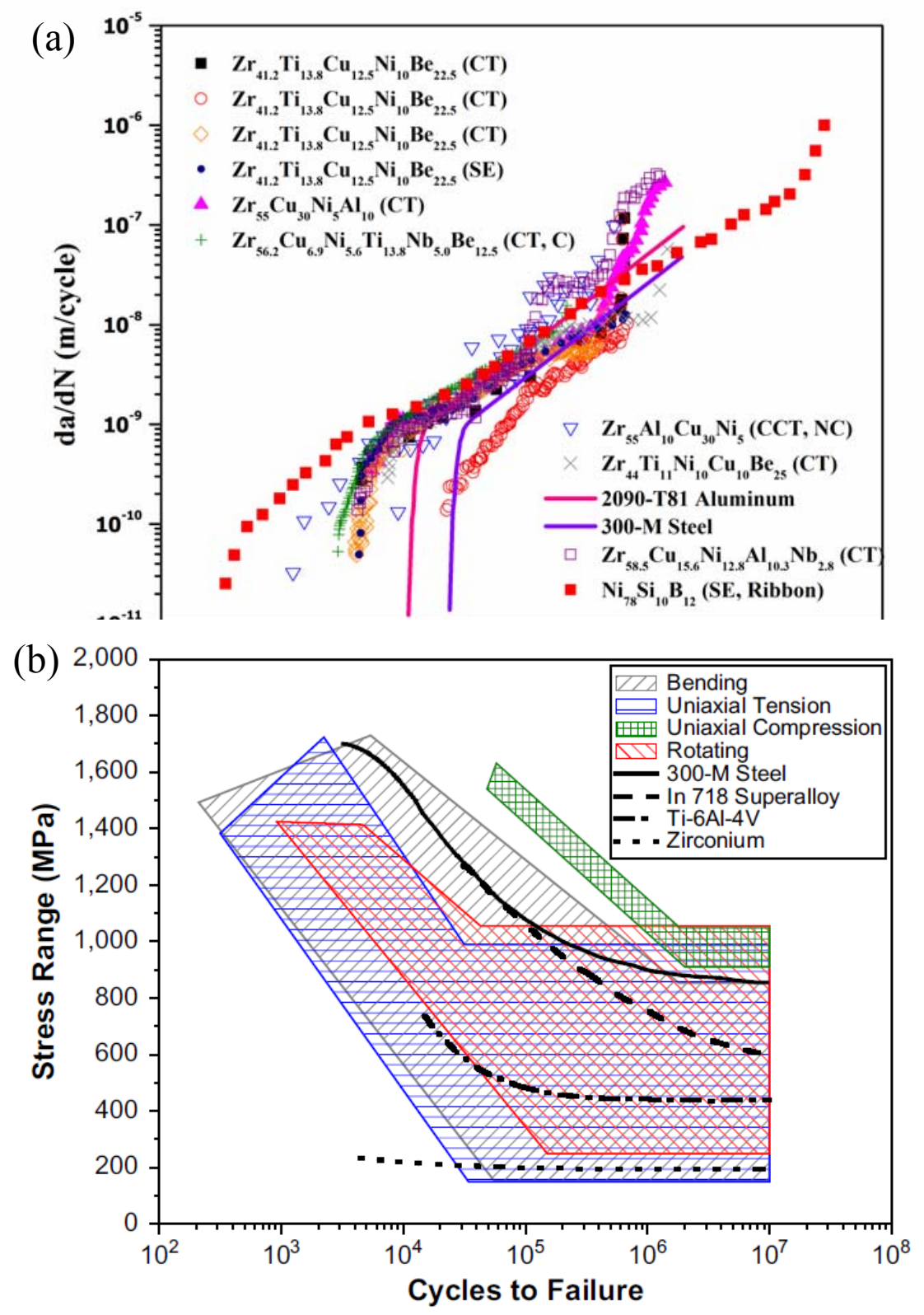
Figure 57 (Continued)

(c)

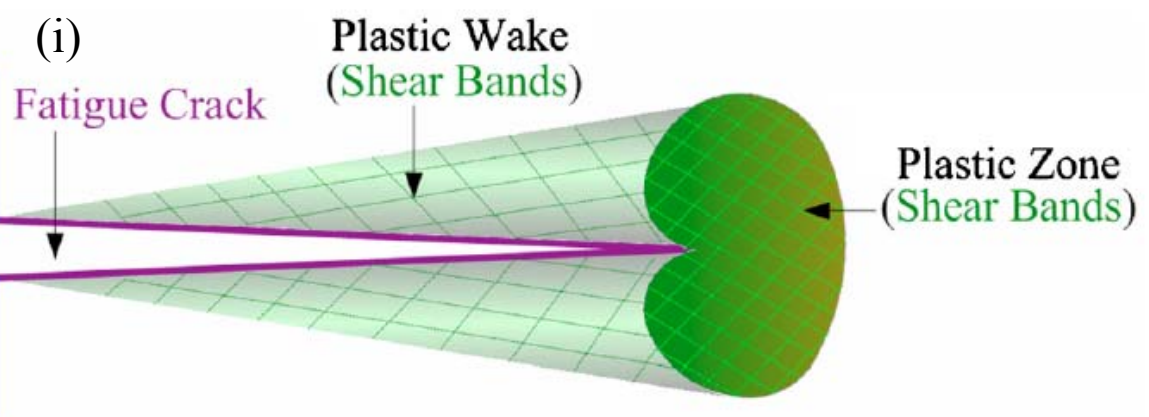

(ii)

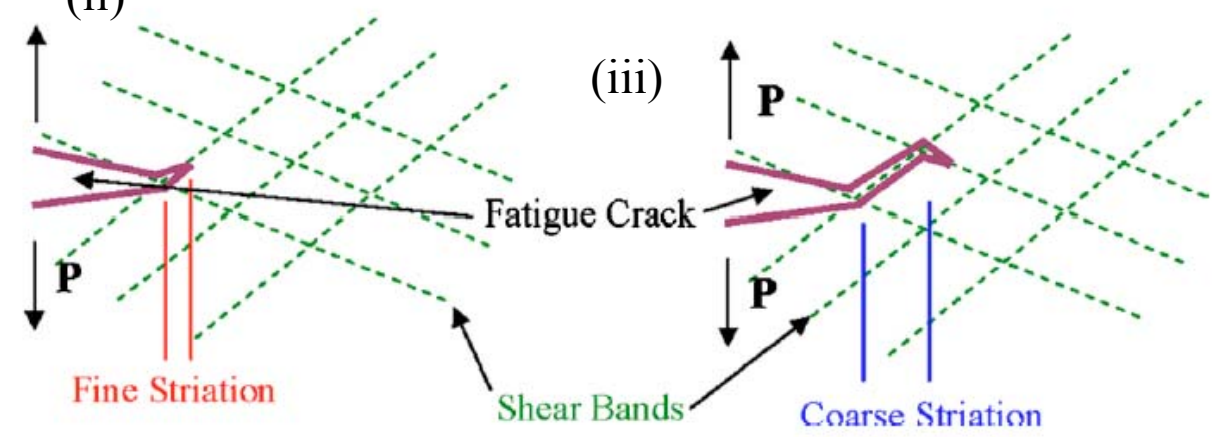


Figure 58 MD simulations on fatigue tests of Al-Fe metallic glass. Two identical specimens were tested under cyclic straining and monotonic loading. (a) The crystalline phase can be seen clearly after 275 cycles; (b) The sample remained amorphous under monotonic loading even though the strain reached $8 \%$; (c) The distribution of the atom fraction vs. the square of nanoaffine displacement $\left(D^{2}\right)$ for cycle nos. 1, 20, 70, 175, and 275; and (d) Both the averaged $D^{2}$ (solid black line) and the average grain size (curve with circles) increased with increasing cycle number [448].
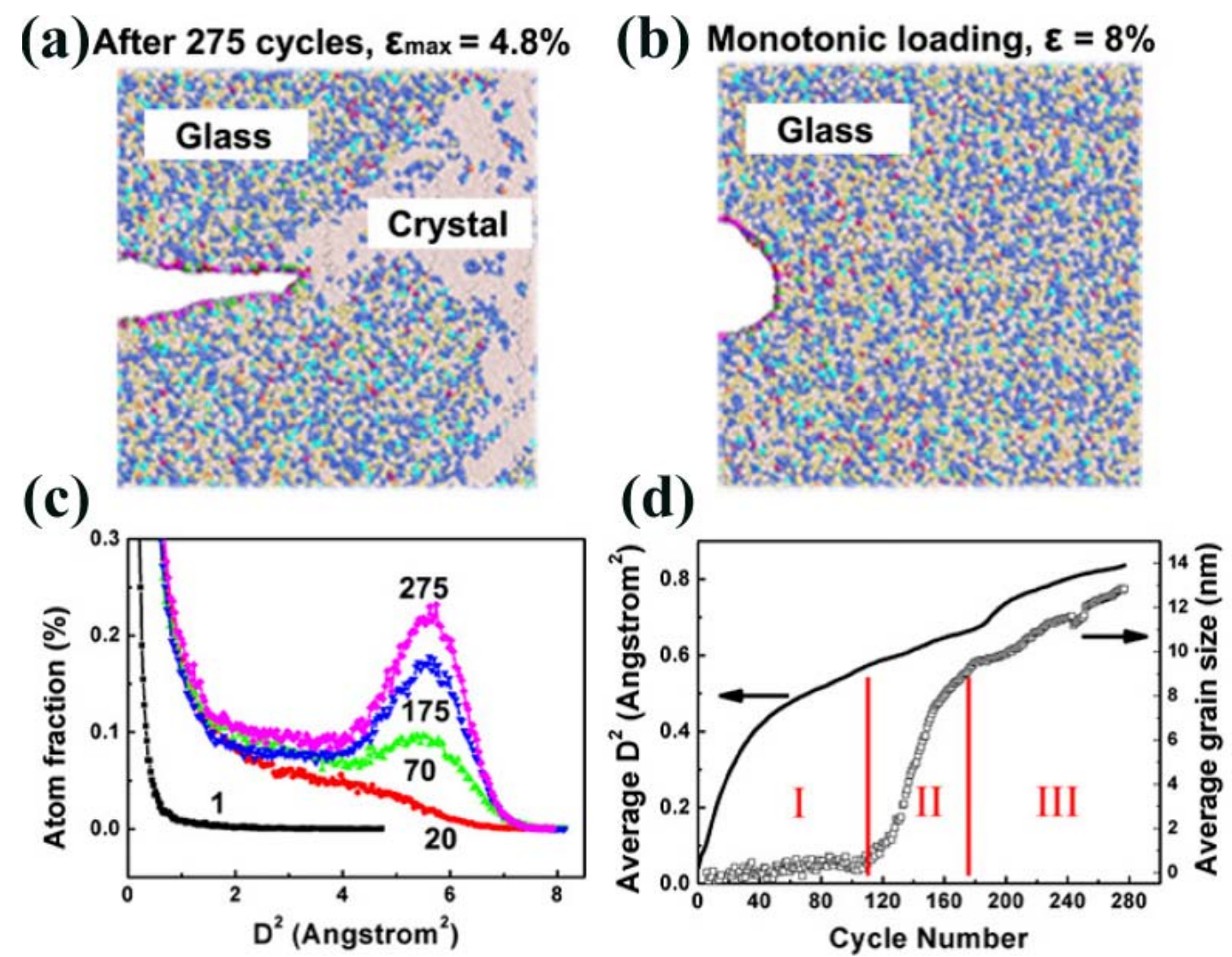
Figure 59 (a) The friction coefficient and (b) wear rate of the

$\mathrm{Zr}_{41.2} \mathrm{Ti}_{13.8} \mathrm{Cu}_{12.5} \mathrm{Ni}_{10} \mathrm{Be}_{22.5} \mathrm{BMG}$ as a function of normal load, and (c) the friction coefficient as a function of sliding speed [457].
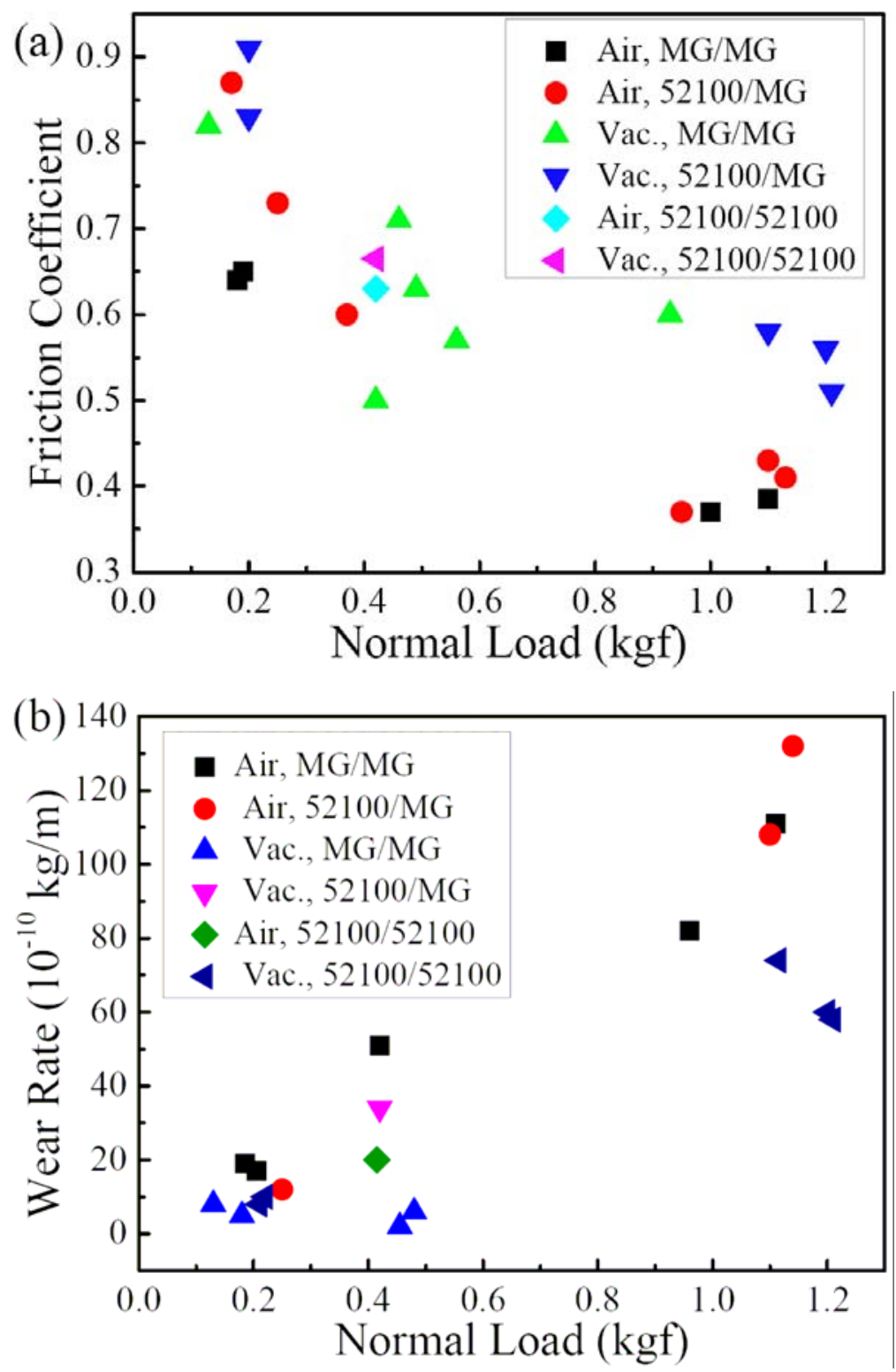


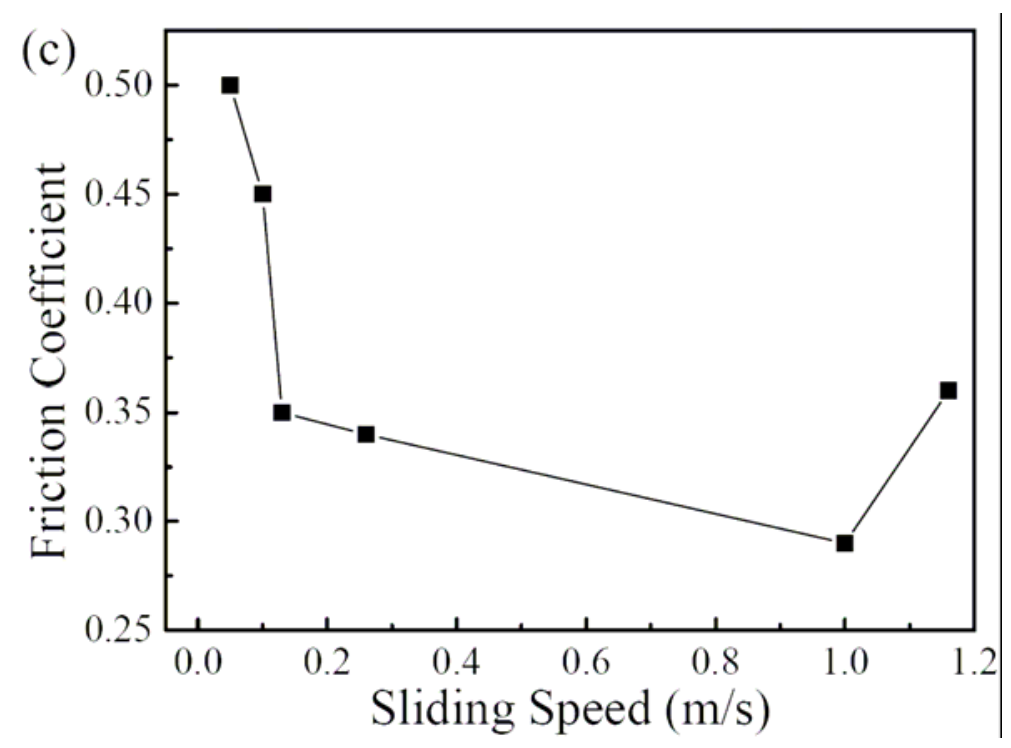


Figure 60 The wear volume with the sliding distance in four BMGs (a) [465] and the wear rate of the $\mathrm{Zr}_{41.2} \mathrm{Ti}_{13.8} \mathrm{Cu}_{12.5} \mathrm{Ni}_{10} \mathrm{Be}_{22.5} \mathrm{BMG}$ with the sliding distance (b) [466].
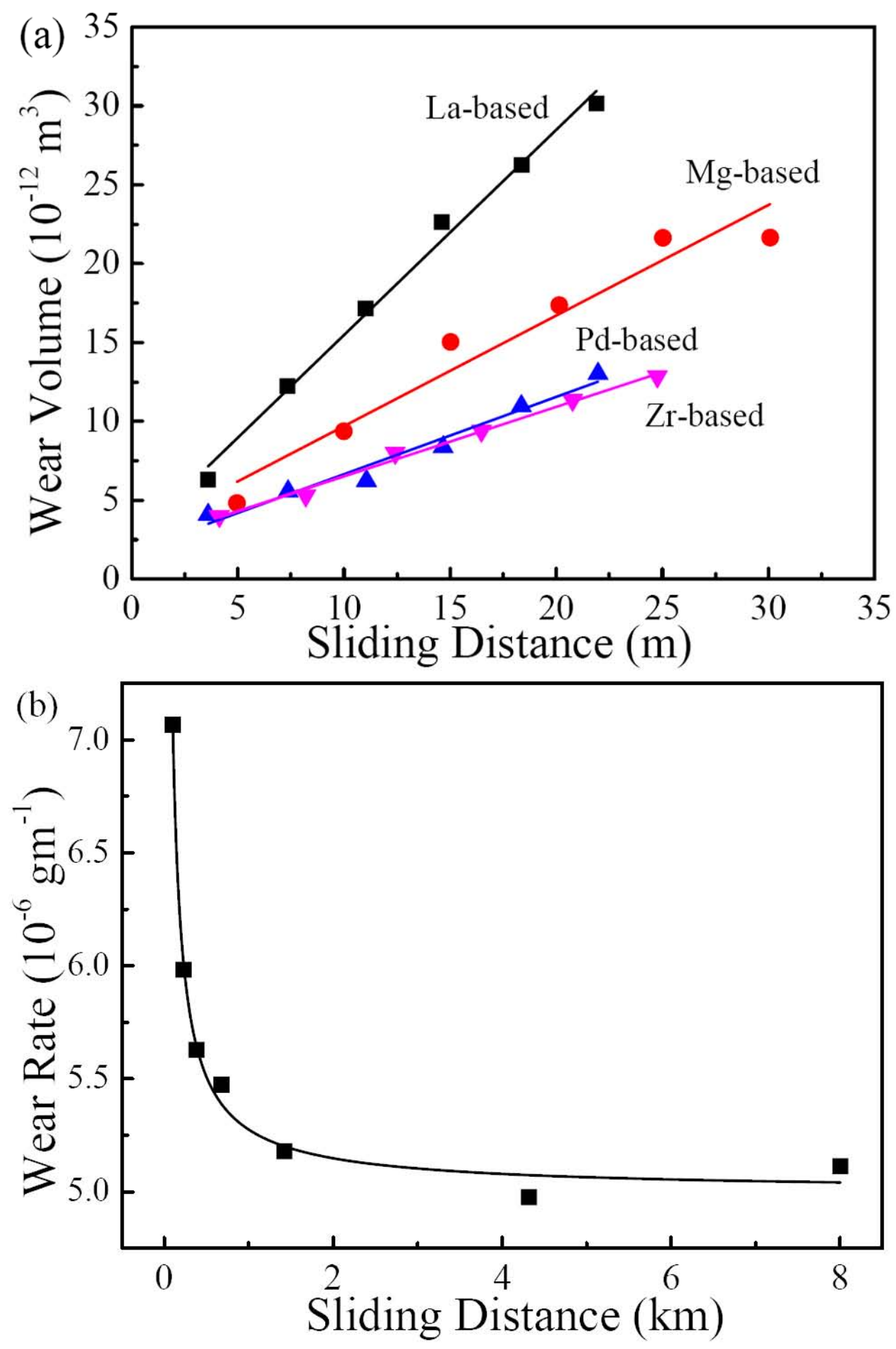
Figure 61 (a) Bright-field TEM image of subsurface region in wear pin for test performed in oxygen; and (b) close-up view of the near-surface region in (a) [473].

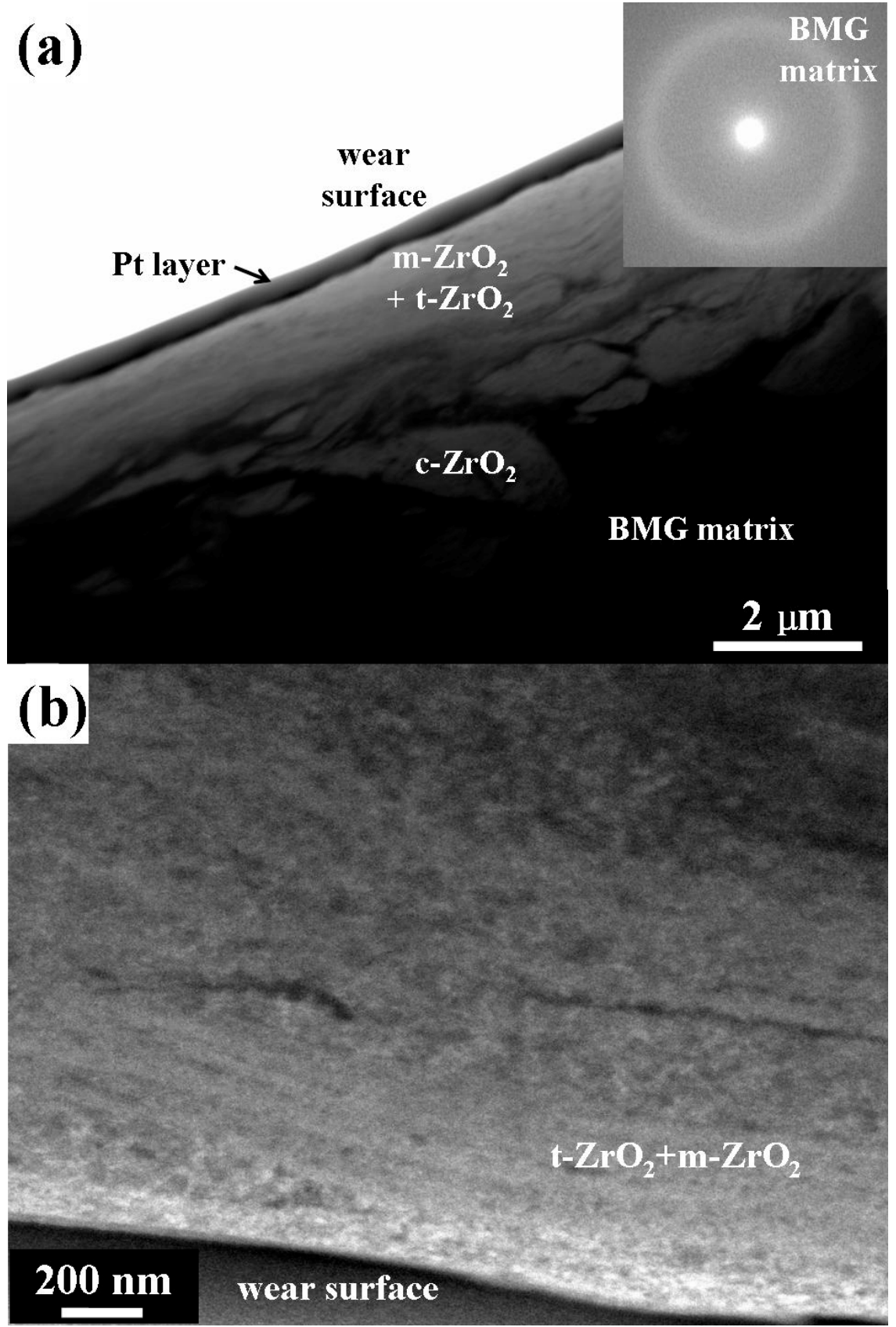


Figure 62 The friction coefficient as a function of sliding time for in-situ dendrite / Zr-based MGMCs under different normal loads (a); and sliding velocities (b) [479].
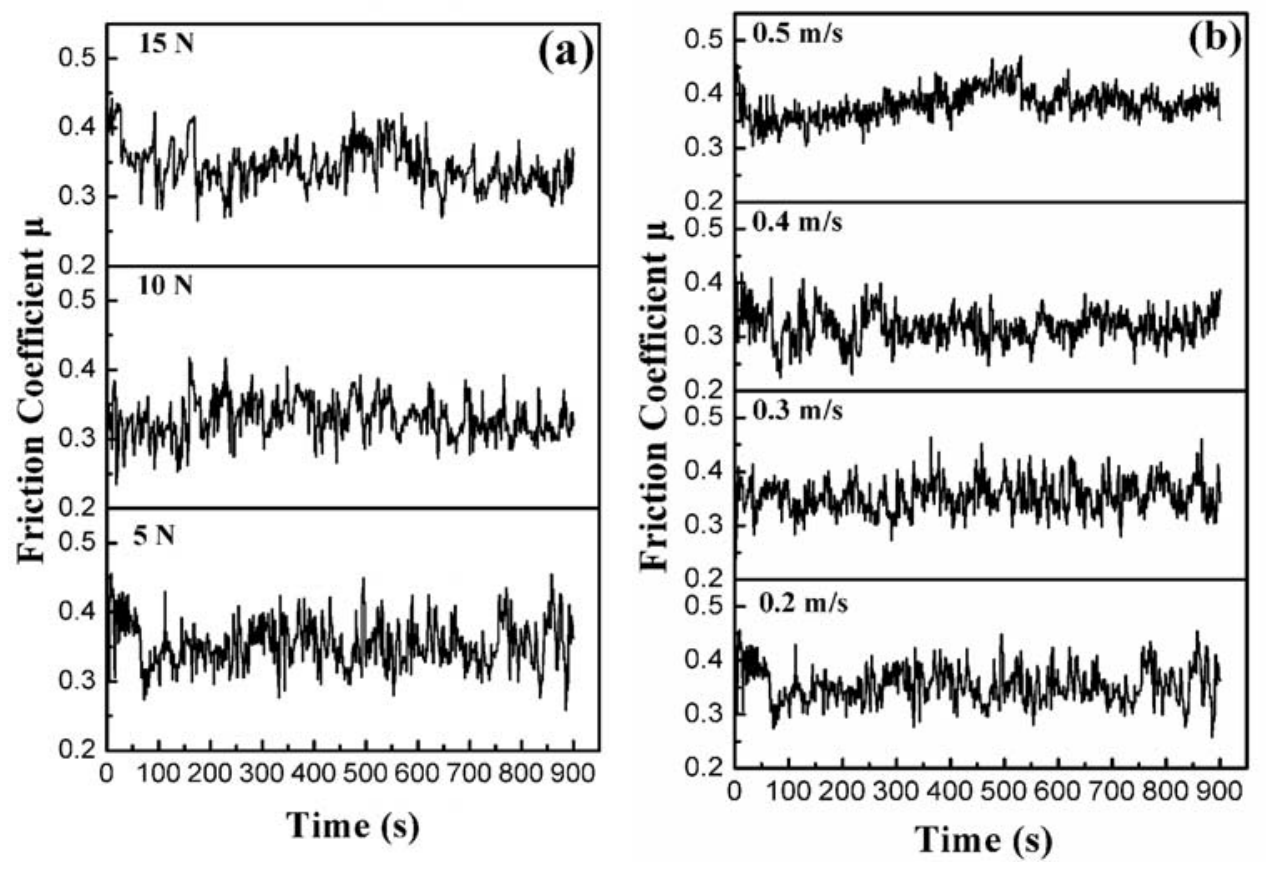
Figure 63 Polarization curves of the $\mathrm{Ti}_{40} \mathrm{Zr}_{24} \mathrm{~V}_{12} \mathrm{Cu}_{5} \mathrm{Be}{ }_{19} \mathrm{MGs}$ in different solutions [490].

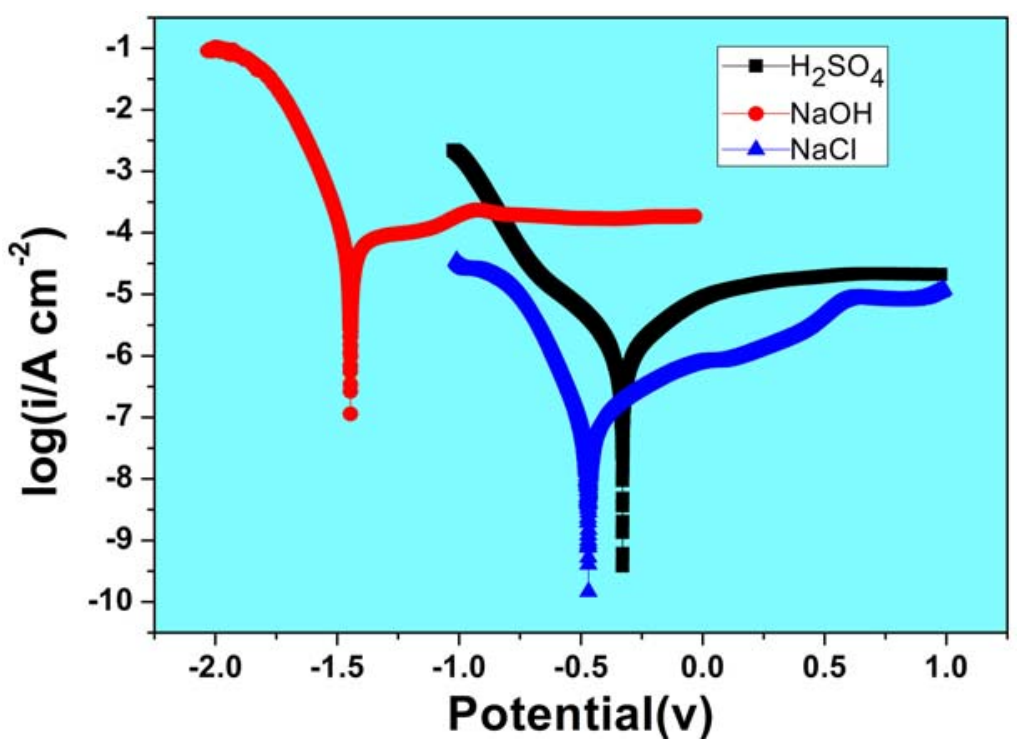


Figure 64 Schematic illustration of the compositional depth profiles of Ti and $\mathrm{Zr}$ oxidized states in passive films for the $\mathrm{Ti}_{46} \mathrm{Zr}_{20} \mathrm{~V}_{12} \mathrm{Cu}_{5} \mathrm{Be}_{17} \mathrm{MGs}$ after the elechemical corrosion experiment in an acid solution [491].

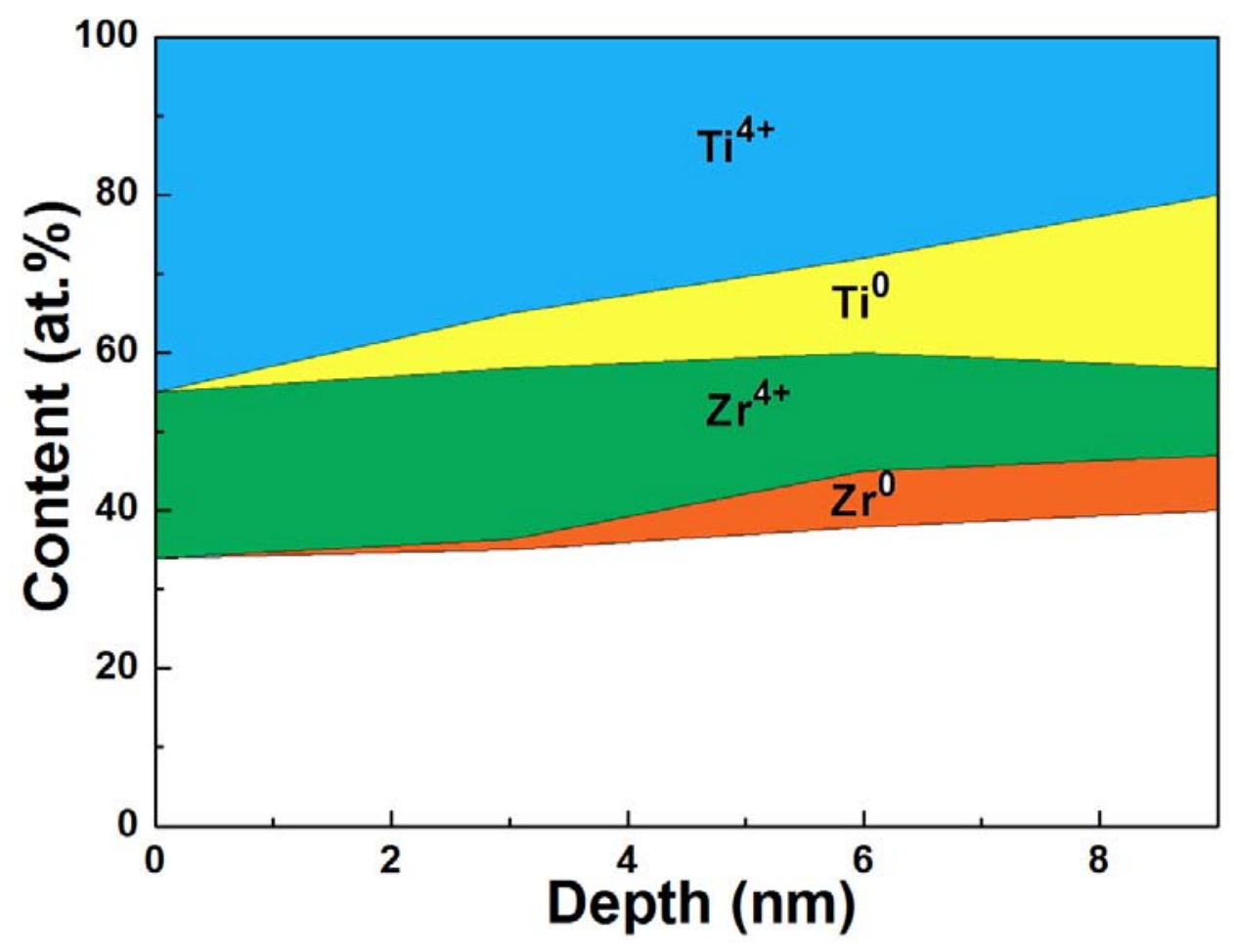


Figure 65 Polarization curves of $\mathrm{Zr}$-based MGMCs in different concentrations of $\mathrm{NaCl}$ solutions (a); and electrochemical impedance spectroscopy (EIS) of Zr-based MGMCs at an open circuit potential in different concentrations of $\mathrm{NaCl}$ solutions (b) [492].
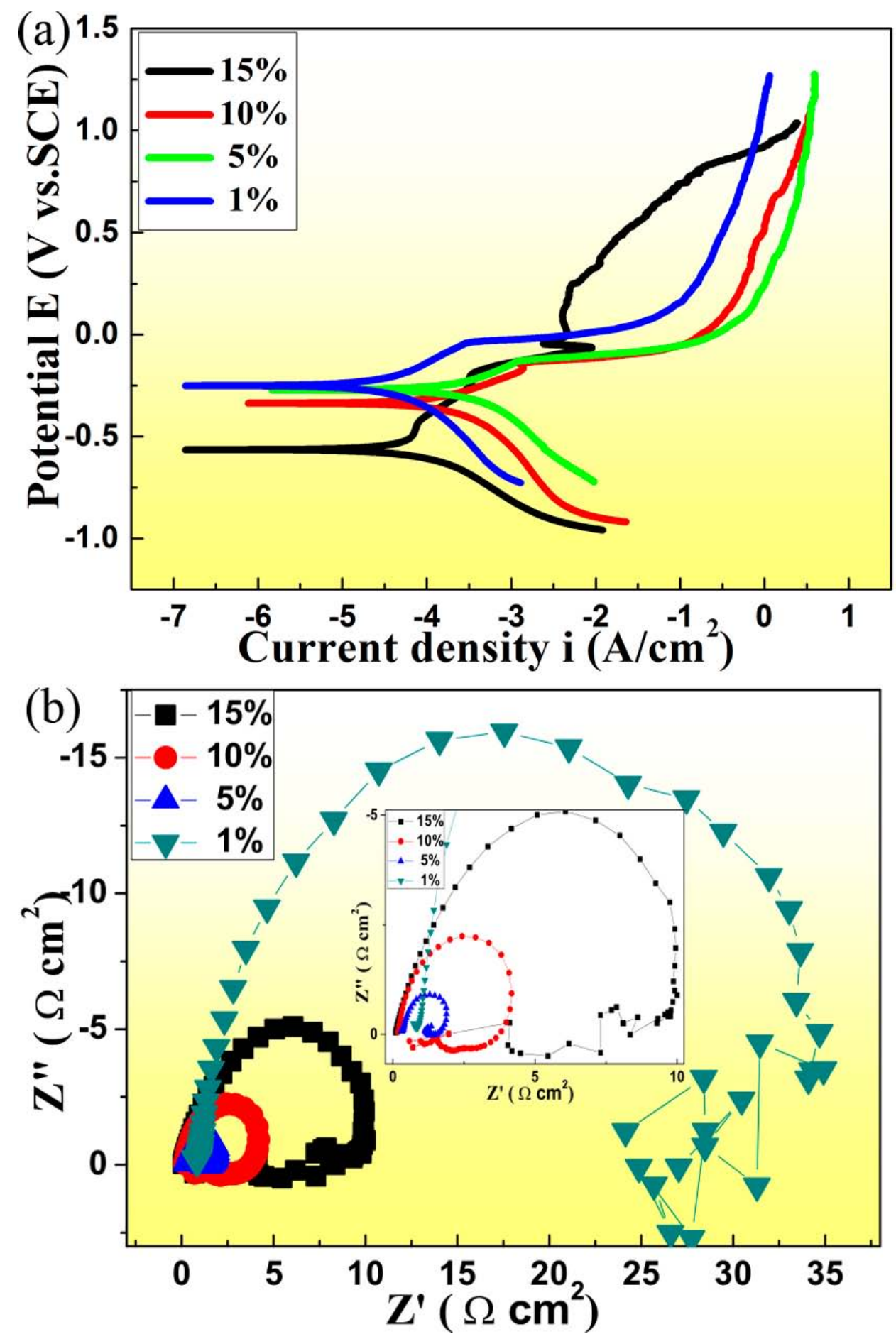
Figure 66 A review of the ductility and yield strength of different kinds of coatings, including TFMGs, metallic coatings, and ceramic coatings applied to improve mechanical properties of substrate materials [438].

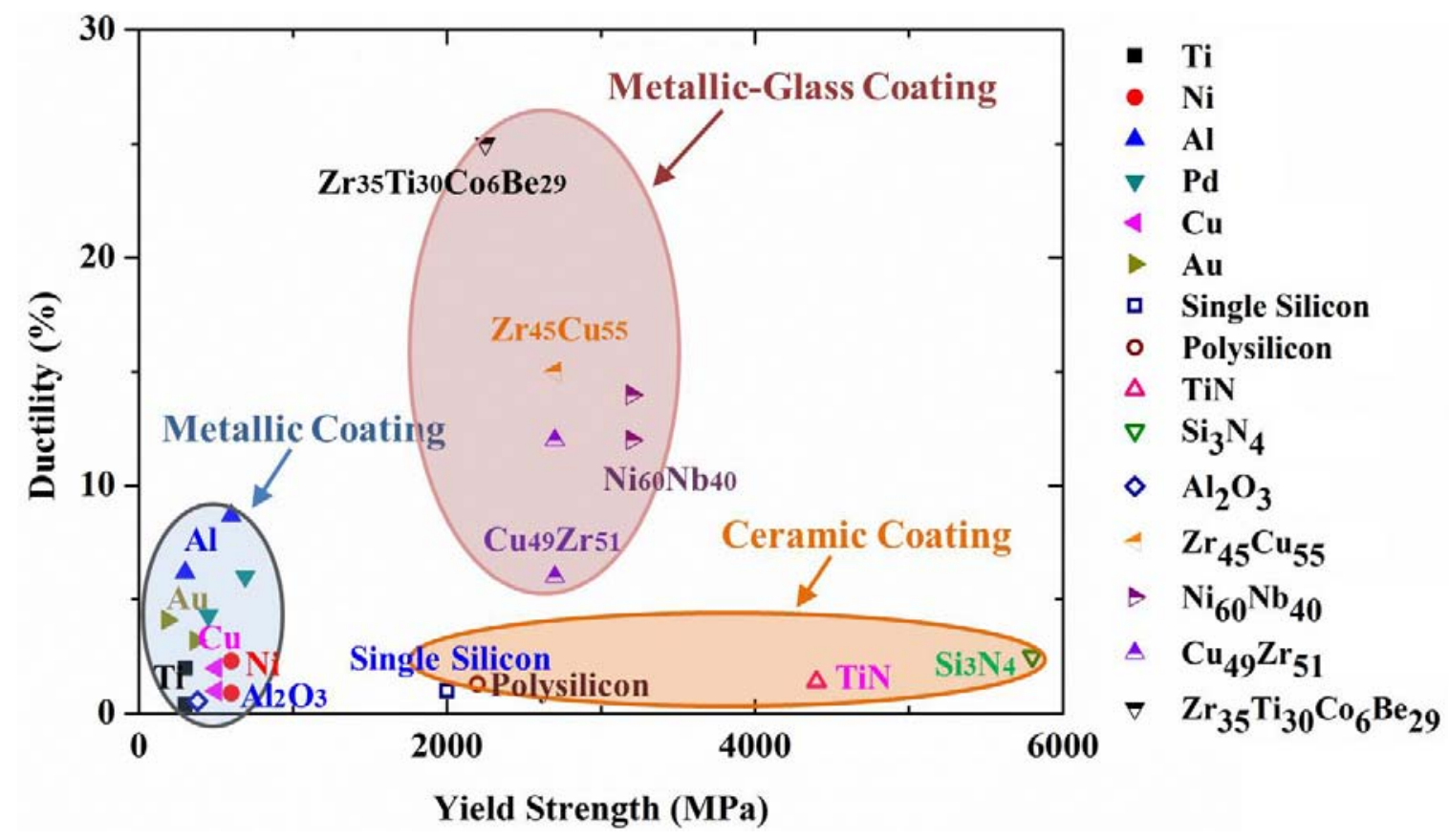


Figure 67 (a) S-N curves for all the reported TFMGs on different kinds of substrates. Five regions are separated in (a) by: A) low fatigue-endurance limit region; B) medium fatigue-endurance limit region; C) high fatigue-endurance limit region; D) tension-tension region; and E) monolithic BMG region [438]; and (b) the improvement of fatigue-endurance limit vs. UTS for all the reported substrate materials, including the 316L steel, Ni-based alloy, Zr-based alloy, Ti-based alloy, and Al-based alloy [438].

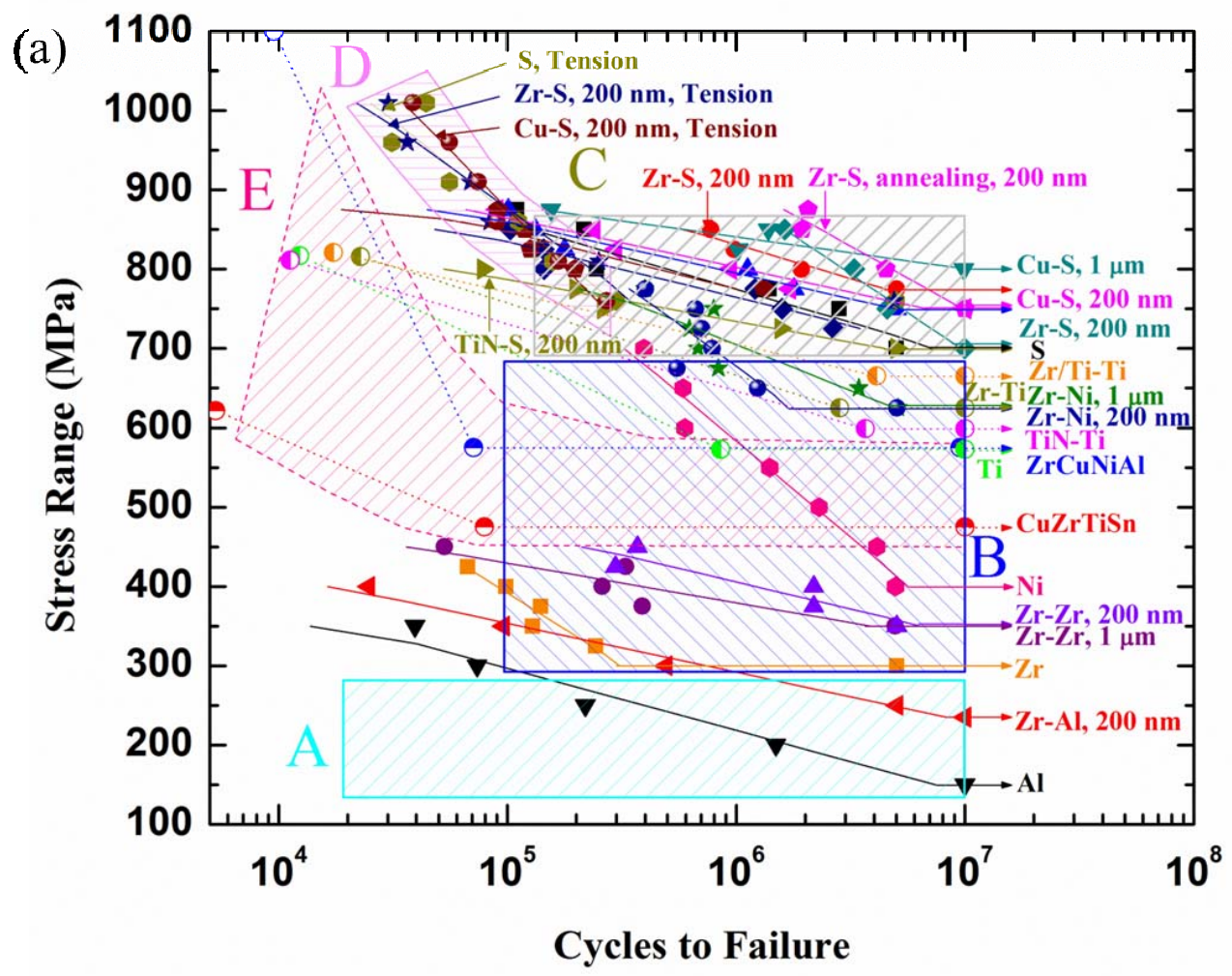

- Bare 316L stainless steel (S), bending [25]

- Zr-based TFMG coated steel (Zr-S), $1 \mu \mathrm{m}$

v Bare Al alloy (7075-T6) (Al) [30]

- Zr-based $200 \mathrm{~nm}[25$

$\nabla$ Cu-based TFMG coated steel (Cu-S), $1 \mu \mathrm{m}$

4 Zr-based TFMG coated Al (Zr-Al), $200 \mathrm{~nm}[30]$

- Zr-S, as-deposit, $200 \mathrm{~nm}[23]$

- Zr-S, annealing, $200 \mathrm{~nm}$ [23]

$4 \mathrm{Cu}-\mathrm{S}, 200 \mathrm{~nm}$

- TiN film coated steel (TiN-S), $200 \mathrm{~nm}$

- Pure-Cu film coated steel (PureCu-S), $200 \mathrm{~nm}$

- Bare 316L stainless steel (S), tension

$\star \mathrm{Zr}-\mathrm{S}, 200 \mathrm{~nm}$, tension

- $\mathrm{Cu}-\mathrm{S}, 200 \mathrm{~nm}$, tension

- Fe-based TFMG coated steel (Fe-S), $200 \mathrm{~nm}$

- Bare C-2000 Ni-based alloy (Ni) [50]

-... BMG Zr55Cu30Ni5Al10 (ZrCuNiAl) [87]

* Zr-based TFMG coated Ni (Zr-Ni), $1 \mu \mathrm{m}$ [50]

- $\mathrm{Zr}-\mathrm{Ni}, 200 \mathrm{~nm}[50]$

- Bare Zr-based alloy (Zr95.sHf4.5) (Zr)

- Zr-based TFMG coated $\mathrm{Zr}(\mathrm{Zr}-\mathrm{Zr}), 1 \mu \mathrm{m}$

- $\mathrm{Zr}-\mathrm{Zr}, 200 \mathrm{~nm}$

๑ $\cdots$ BMG (Cu60Zr30Ti10)99Sn1 (CuZrTiSn) [87]

- D... Bare Ti-6Al-4V (Ti) [69]

- … TiN coated Ti (TiN-Ti) [69]

- - Zr-based TFMG coated Ti (Zr-Ti) [69]

- D Zr-based TFMG/Ti coated Ti (Zr/Ti-Ti) [69] 


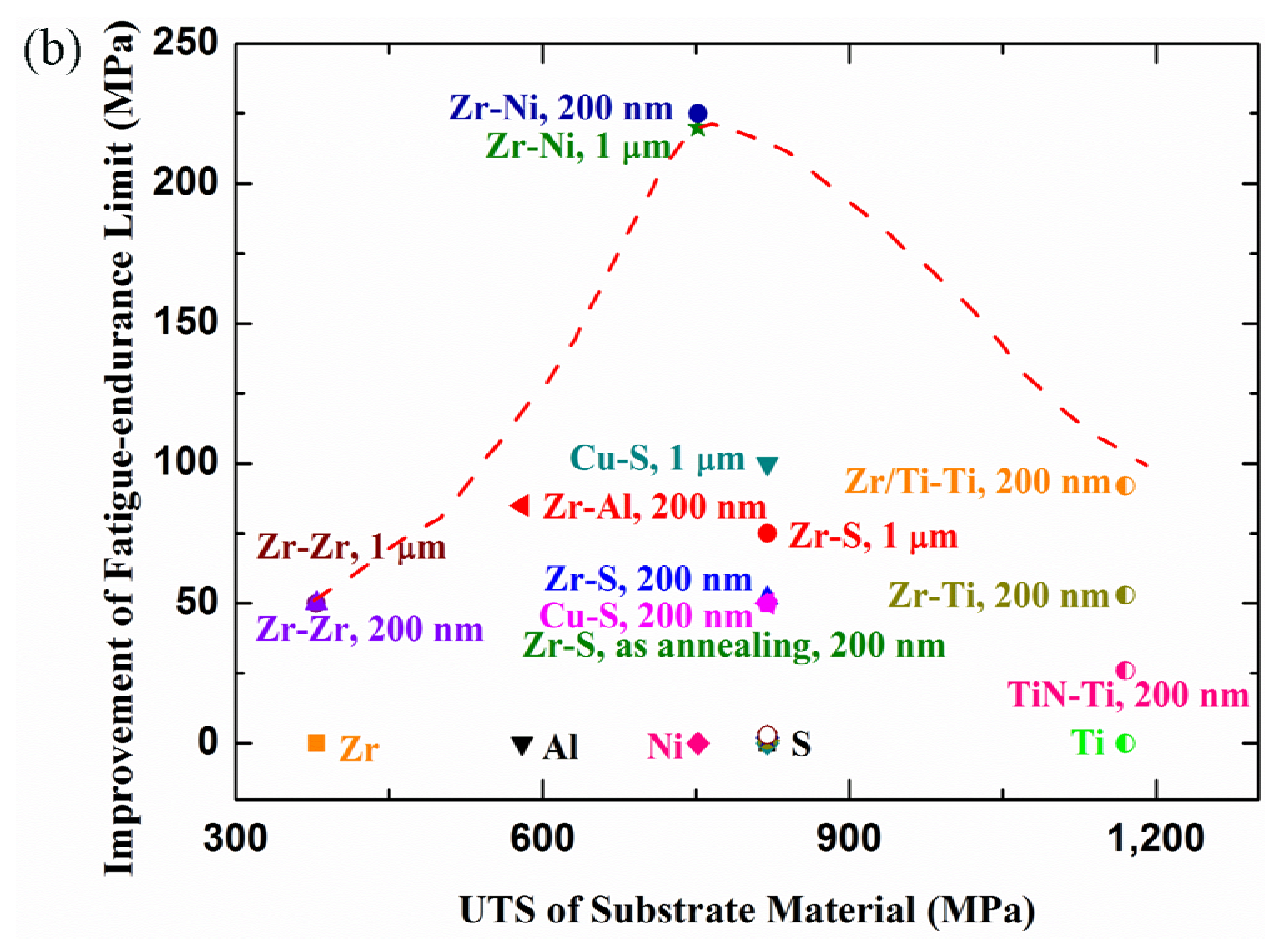


Figure 68 TEM bright-field images and diffraction patterns from Fe-based TFMGs revealing phase evolution during 1 min annealing: amorphous matrix with $\gamma$-fcc FeNi nanocrystallites (as-deposited) $\rightarrow$ growth of nanocrystals (up to $400{ }^{\circ} \mathrm{C}$ ) $\rightarrow$ amorphous $\left(500^{\circ} \mathrm{C}\right.$ and 550 $\left.{ }^{\circ} \mathrm{C}\right) \rightarrow$ formation of cubic $\mathrm{Fe}(\mathrm{Ni})$ crystals $\left(600^{\circ} \mathrm{C}\right)$ with minor cubic FeNi phase $\left(650^{\circ} \mathrm{C}\right.$ to 700 $\left.{ }^{\circ} \mathrm{C}\right) \rightarrow$ formation and grain growth of FeNi phase $\left(750^{\circ} \mathrm{C}\right)[525]$.
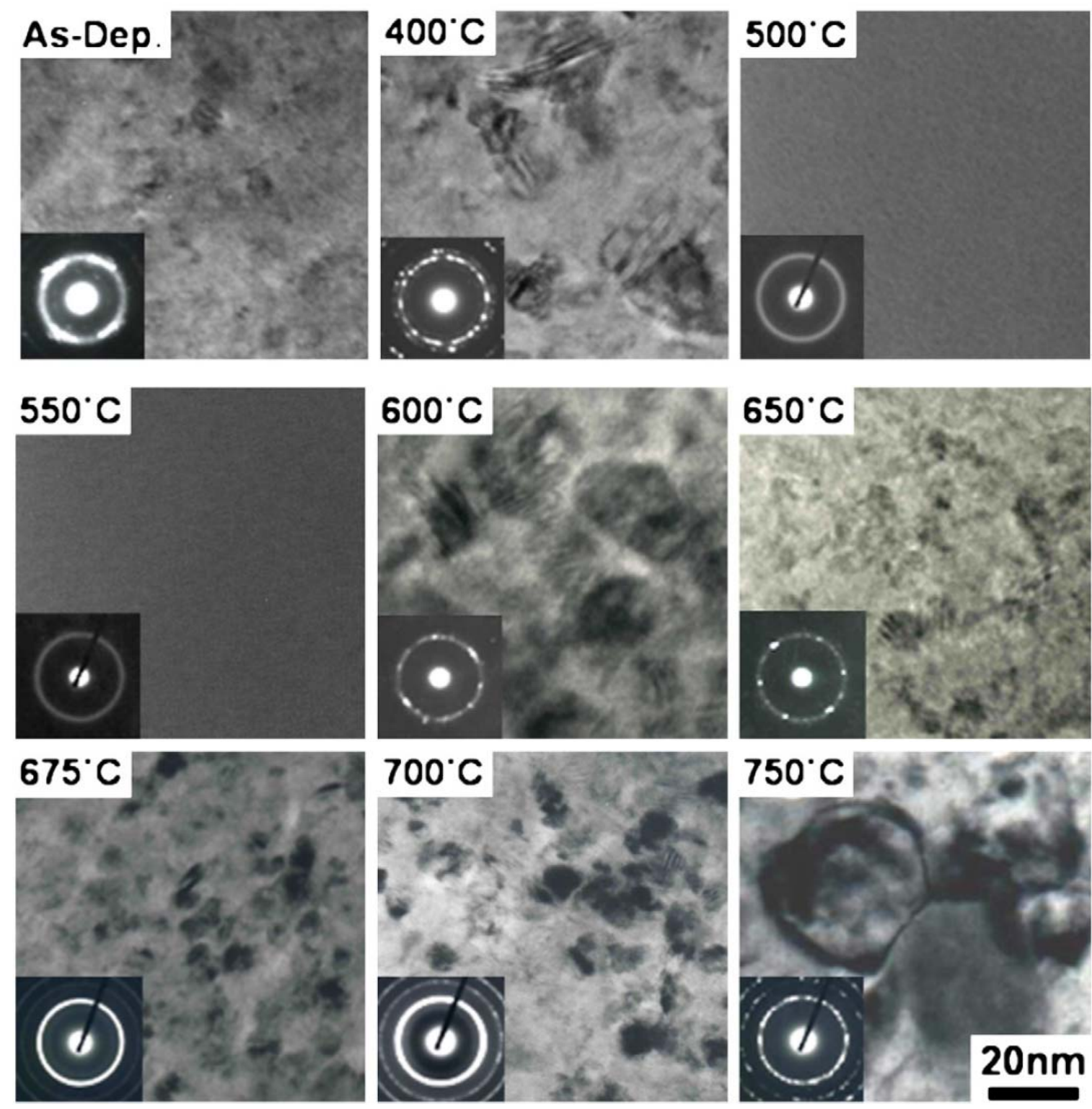
Figure 69 S-N curves of a bare 316L stainless-steel substrate and coated specimens with as-deposited and annealed 200-nm-thick Zr53Cu29A112Ni6 TFMGs under four-point-bending fatigue tests [515].

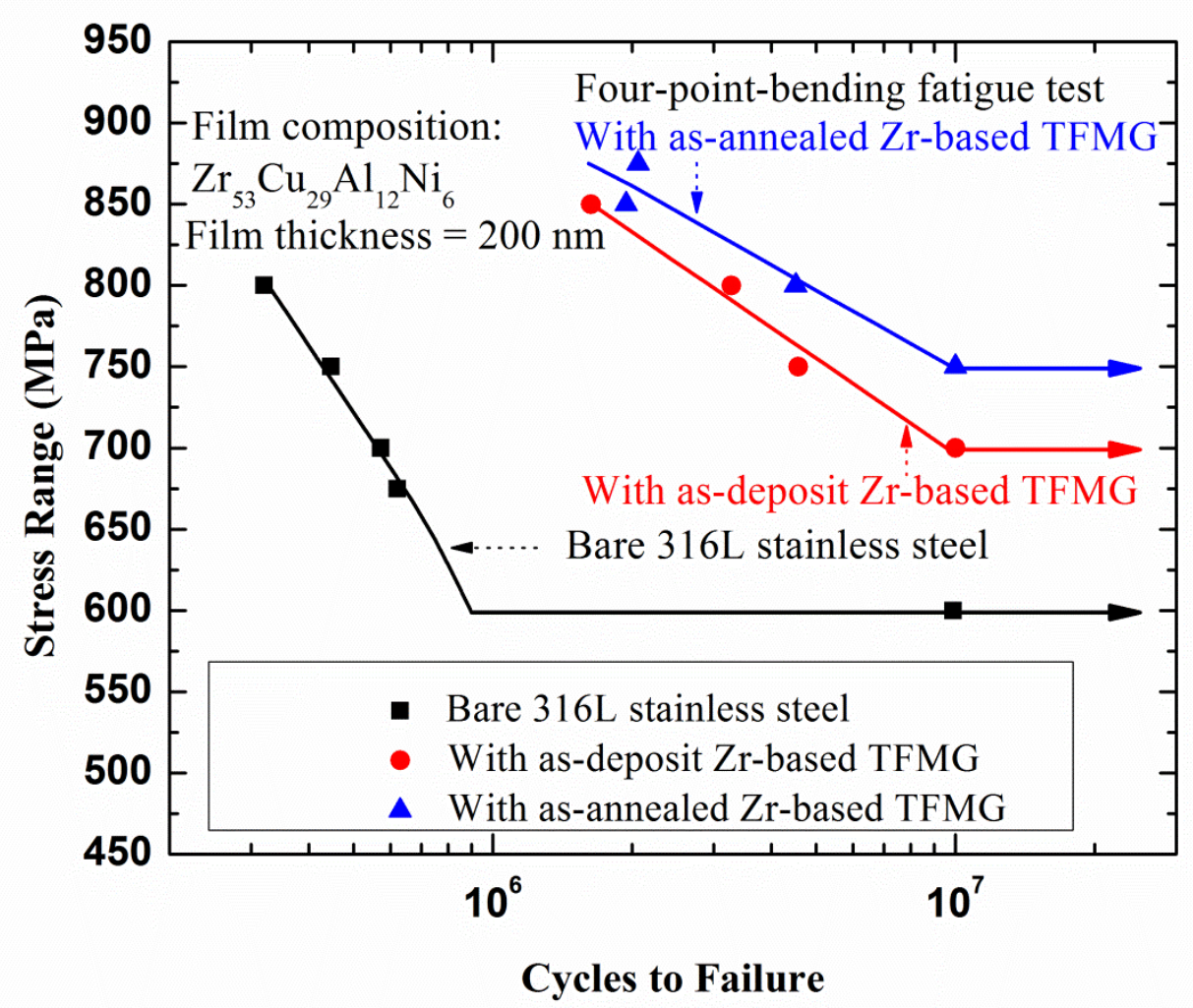


Figure 70 (a) MD Simulated $80 \AA$-thick Cu47Zr47A16 TFMG (Cu atoms = green, $\mathrm{Zr}=$ blue, and $\mathrm{Al}=$ red) on a Ti substrate; and (b) MD simulation of the engineering strain $\varepsilon 11$ under a rigid indent in a Cu47Zr47A16 TFMG on a rigid Ti substrate [515].
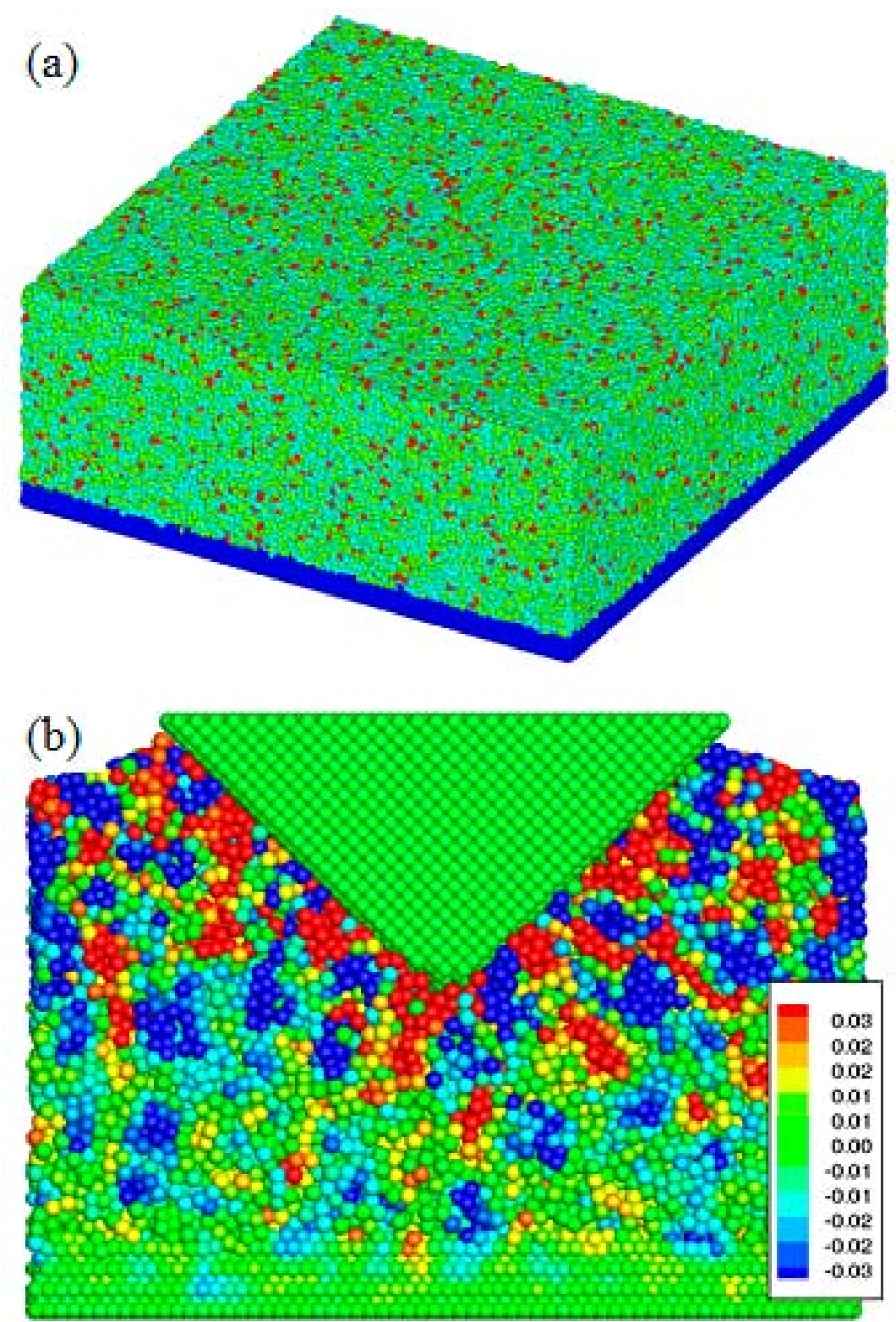
Figure 71 ABAQUS contours of equivalent plastic strains in (a) a bare BMG substrate; and (b) an MG-coated BMG specimen after four-point-bending deformation [438].
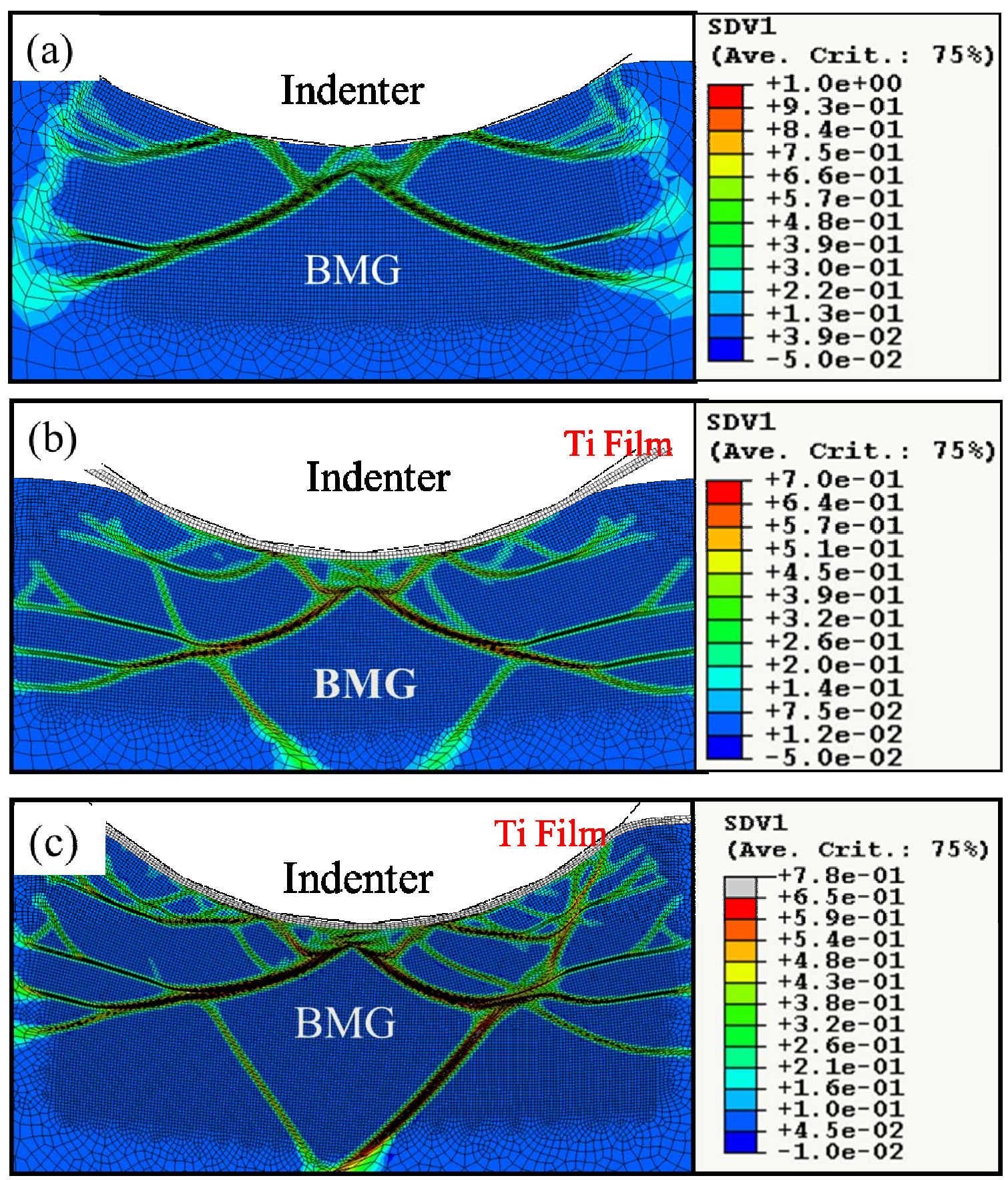
Figure 72 (a) ABAQUS model of a half-symmetric Rockwell indention in a metallic glass, used for predicting shear-band directions in (b) a BMG substrate; and (c) a Ti-coated BMG with a film/substrate thickness ratio of $1 / 20$, having $\mu+\beta=0$ and $v=0.3$ [438].
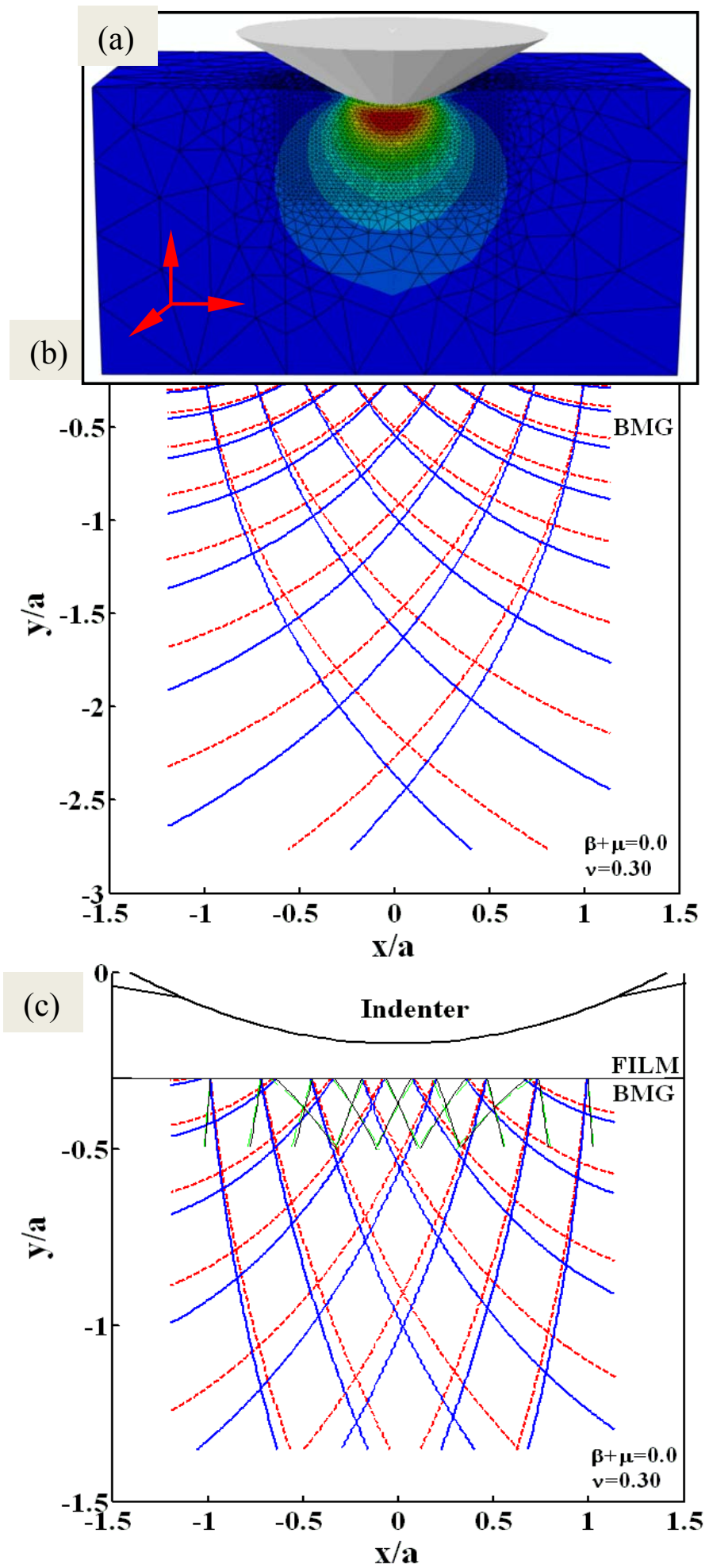
Figure 73 TEM bright-field image and the corresponding SAED pattern (inset) for the dendrites shown in (a); low-magnification HRTEM image of dendrites taken away from the interface showing some Moiré patterns (b); and high-magnification TEM bright-field image showing a step morphology at the interface; HRTEM image close to the interface showing lattice distortion (c); and FFT pattern of the area marked by the rectangle in the inset, the corresponding IFFT pattern shown in (d); HRTEM image of the glass matrix indicating the presence of nano-crystallites (e), the IFFT pattern (inset) of nano-crystallites giving a periodic lattice contrast [544].
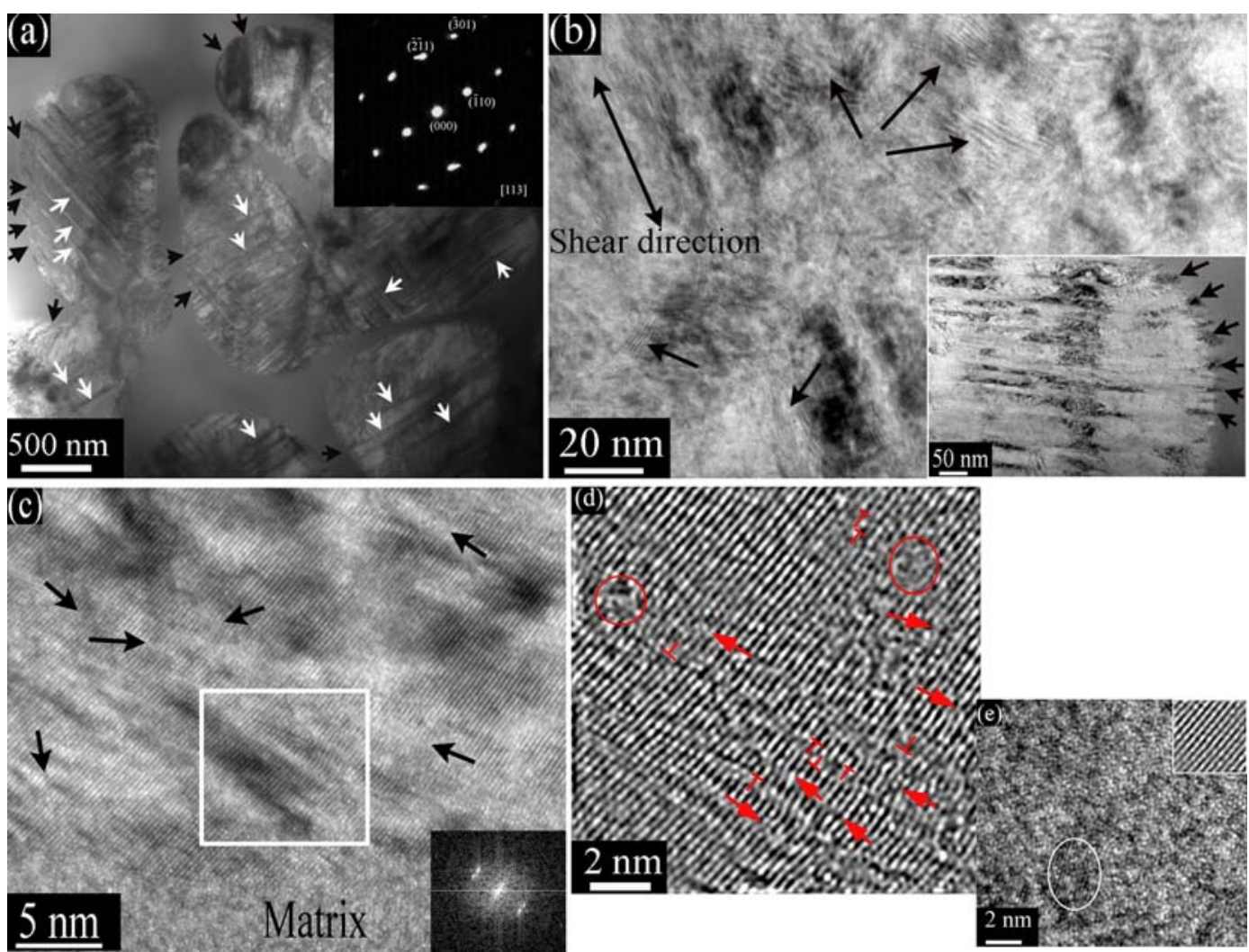
Figure 74 The TEM BF image of the composite with a low magnification shown in a; the HRTEM image of the glass matrix exhibited in $b$ and its corresponding SAED pattern in the inset; the BF and DF images of the deformed dendrites shown in $\mathrm{c}$ and $\mathrm{d}$, respectively; the SAED patterns of the dendrites marked by rectangles in $\mathrm{c}$ and $\mathrm{d}$ shown in the insets of $\mathrm{c}$ and $\mathrm{d}$, respectively; the IFFT image within the shear bands marked by arrows in $\mathrm{c}$ and $\mathrm{d}$ shown in e; the BF and DF images of the dendrites separated by DDWs, denoted by arrows, exhibited in f and g, respectively; the high-magnified $\mathrm{BF}$ image of $\mathrm{CB} \mathrm{B}$, as marked in $\mathrm{f}$ and $\mathrm{g}$, shown in the inset of Figure $5 \mathrm{~g}$; the IFFT and FFT patterns taken from the DDW near the interface of CBs A and $\mathrm{B}$ displayed in $\mathrm{h}$ and the inset of $\mathrm{h}$, respectively; the BF images of the tensile- and shear-induced separations shown in i and j, respectively; the HRTEM image near the interface of the dendrites and glass matrix exhibited in k; and the IFFT image in 1 of the dendrites marked by a rectangle in $\mathrm{k}$ [101].
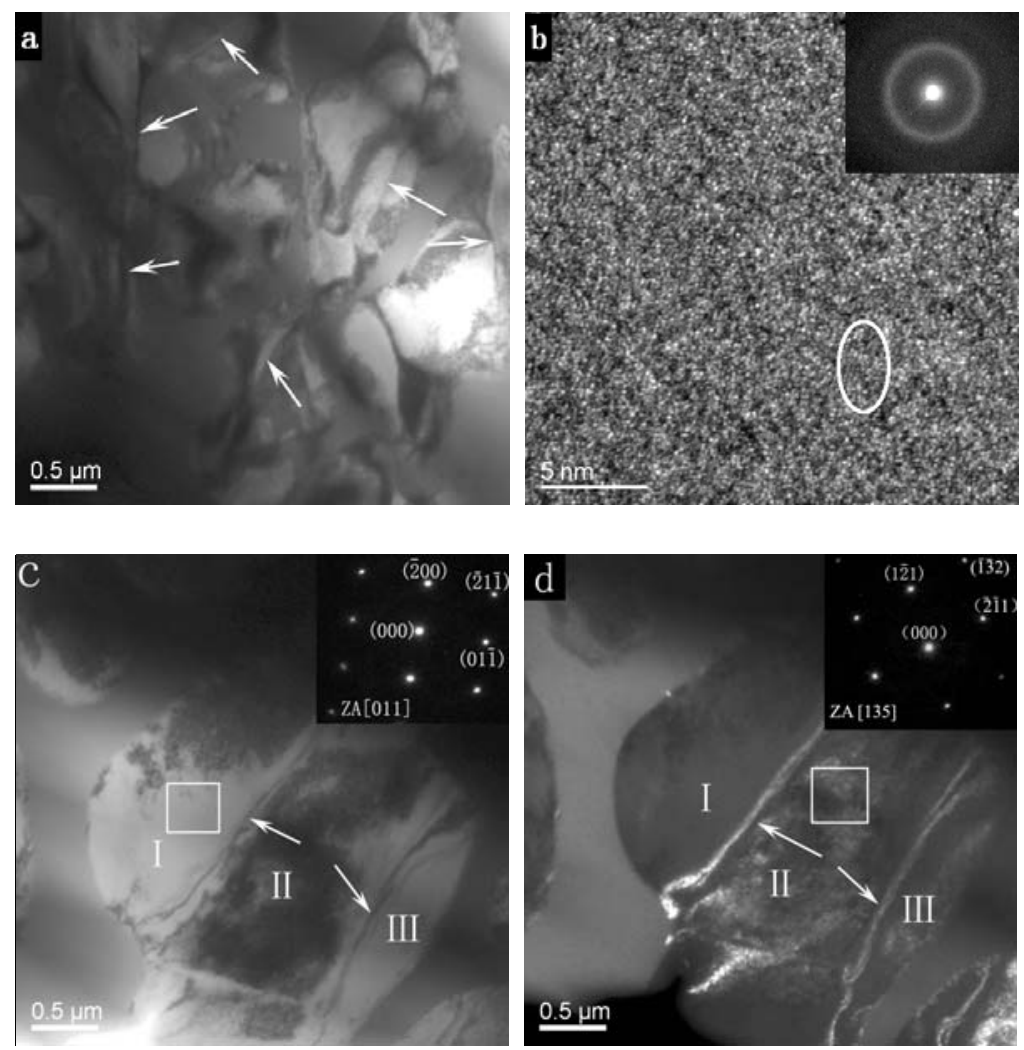

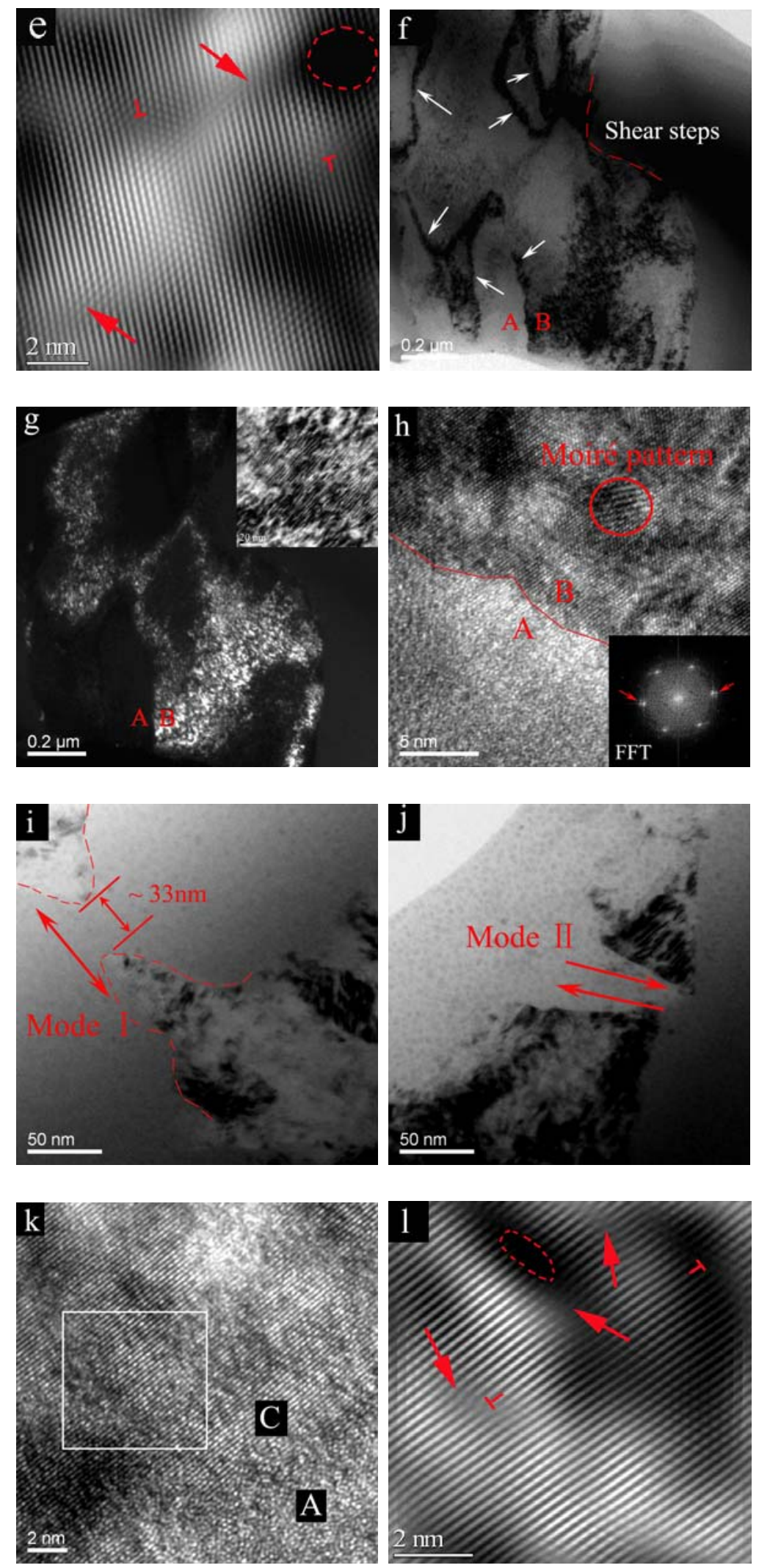
Figure 75 The observation of the crack deflection and propagation between the two phases: (a) from the amorphous phase to the dendrite phase; and (b) from the dendrite phase to the amorphous phase [553].

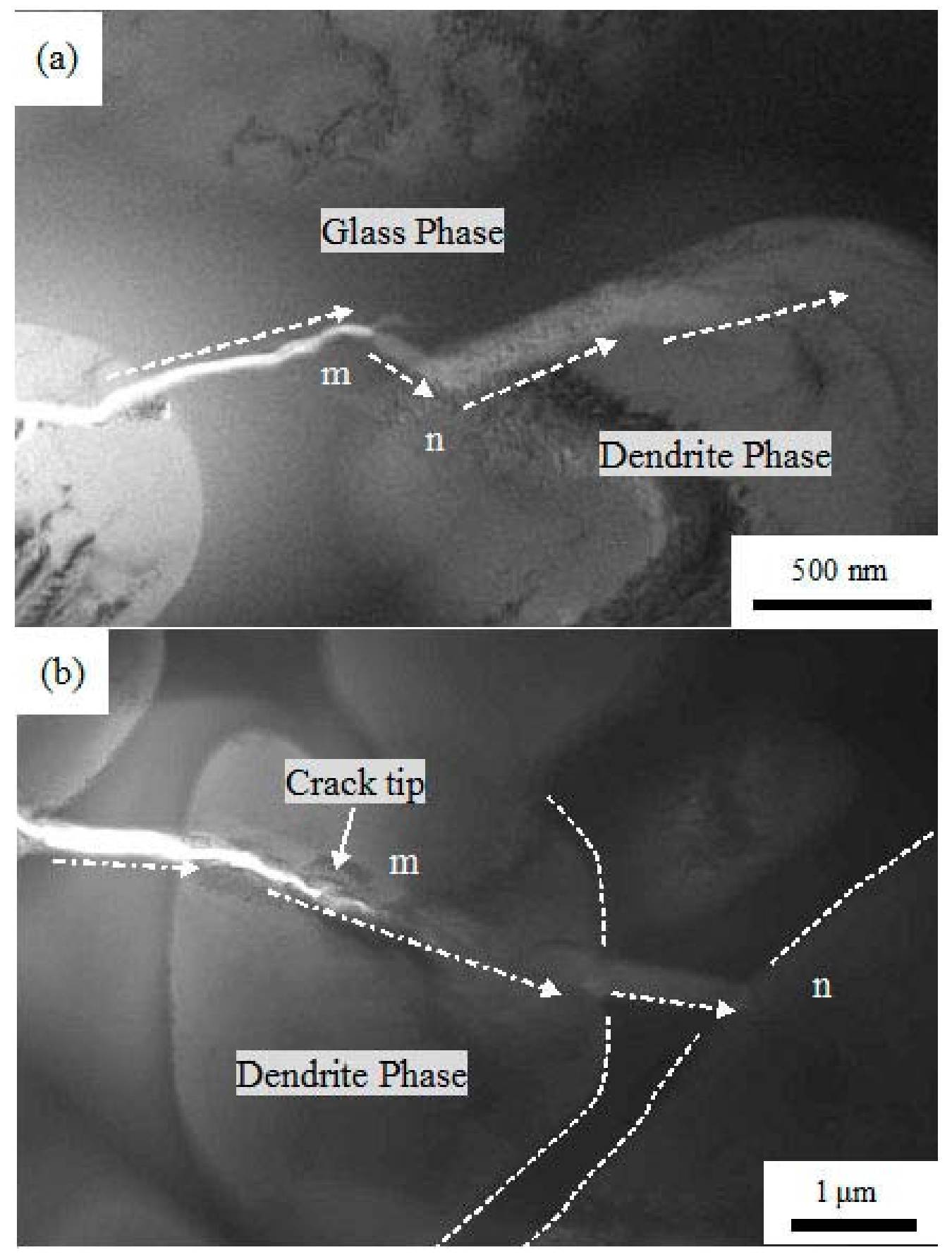


Figure 76 Surface plots of shear stress fields in MGMCs containing dendrites with a fracture energy $e_{\mathrm{c}}=145.2 \mathrm{~J} / \mathrm{m}^{2}$. (a) Rotation angle $\theta=0 \mathrm{deg}$. The color bars marked as $\mathrm{S}_{\mathrm{m}}$ and $\mathrm{S}_{\mathrm{d}}$ at the left are for the maximum shear stresses in the BMG matrix and dendrites, respectively; and (b) Rotation angle $\theta=15$ deg. The color bars marked as $\mathrm{S}_{\mathrm{m}}$ and $S_{d}$ at the right are for the maximum shear stresses in the glass matrix and dendrites, respectively [558].

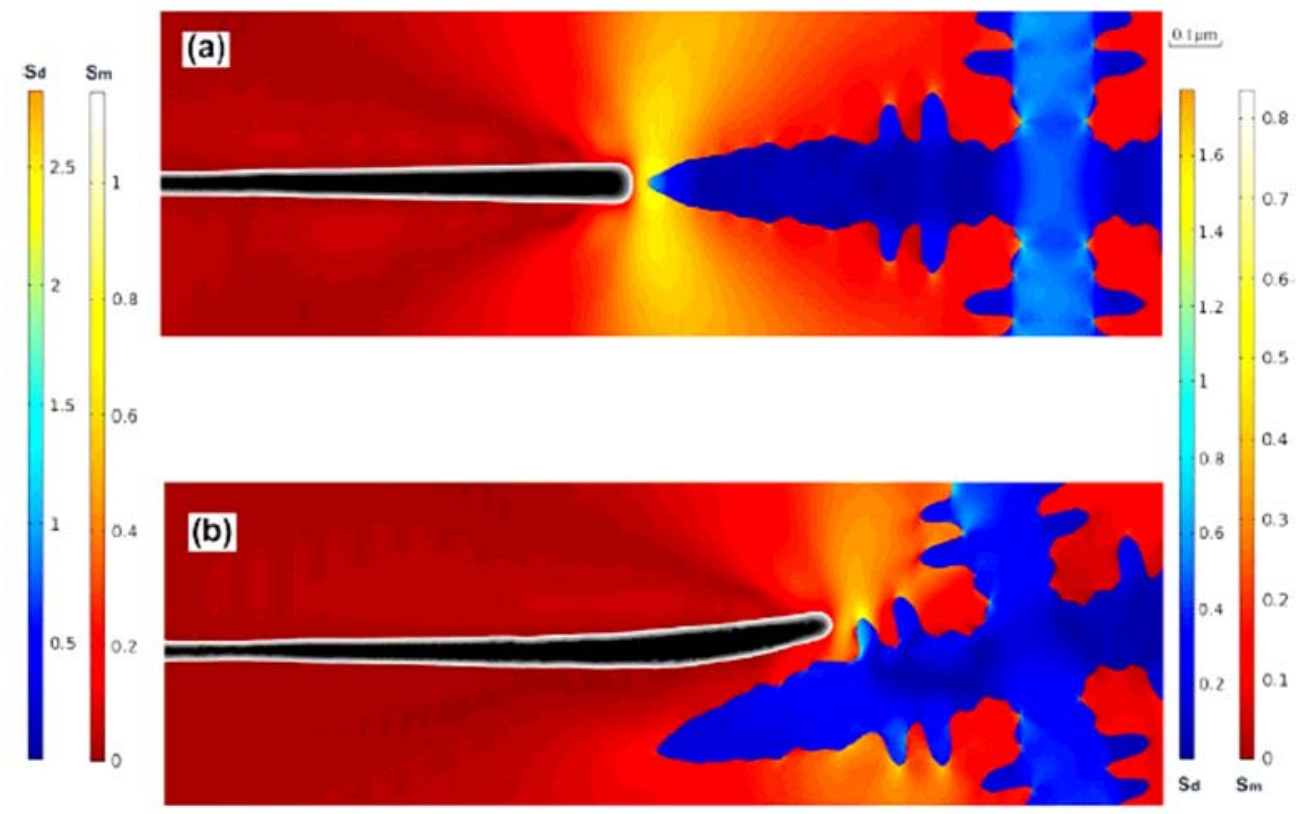


Figure 77 Some BMG products from DongGuan Eontec Co., Ltd. [565].

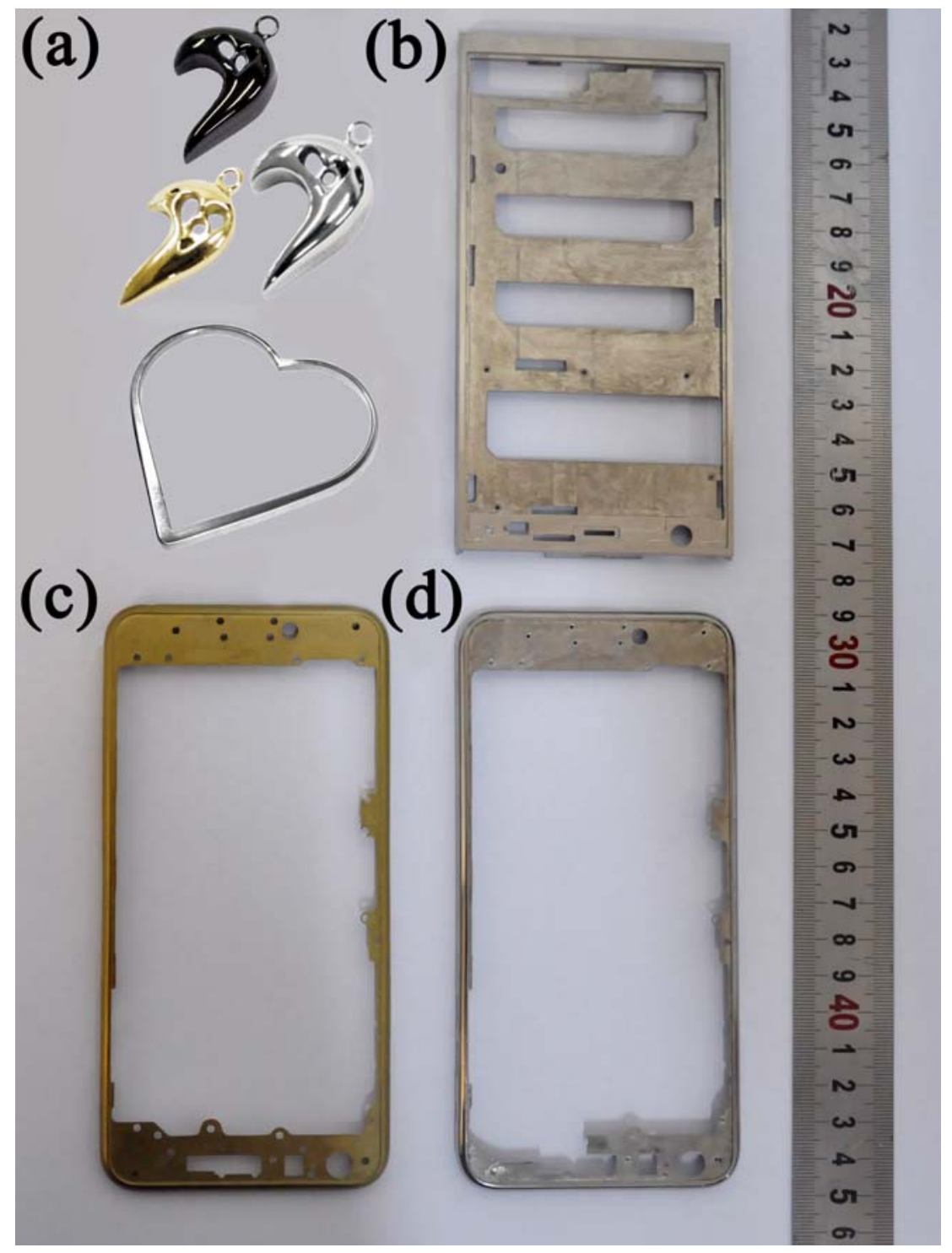


Figure 78 High-performance knives based on Ti-based MGMCs [570].

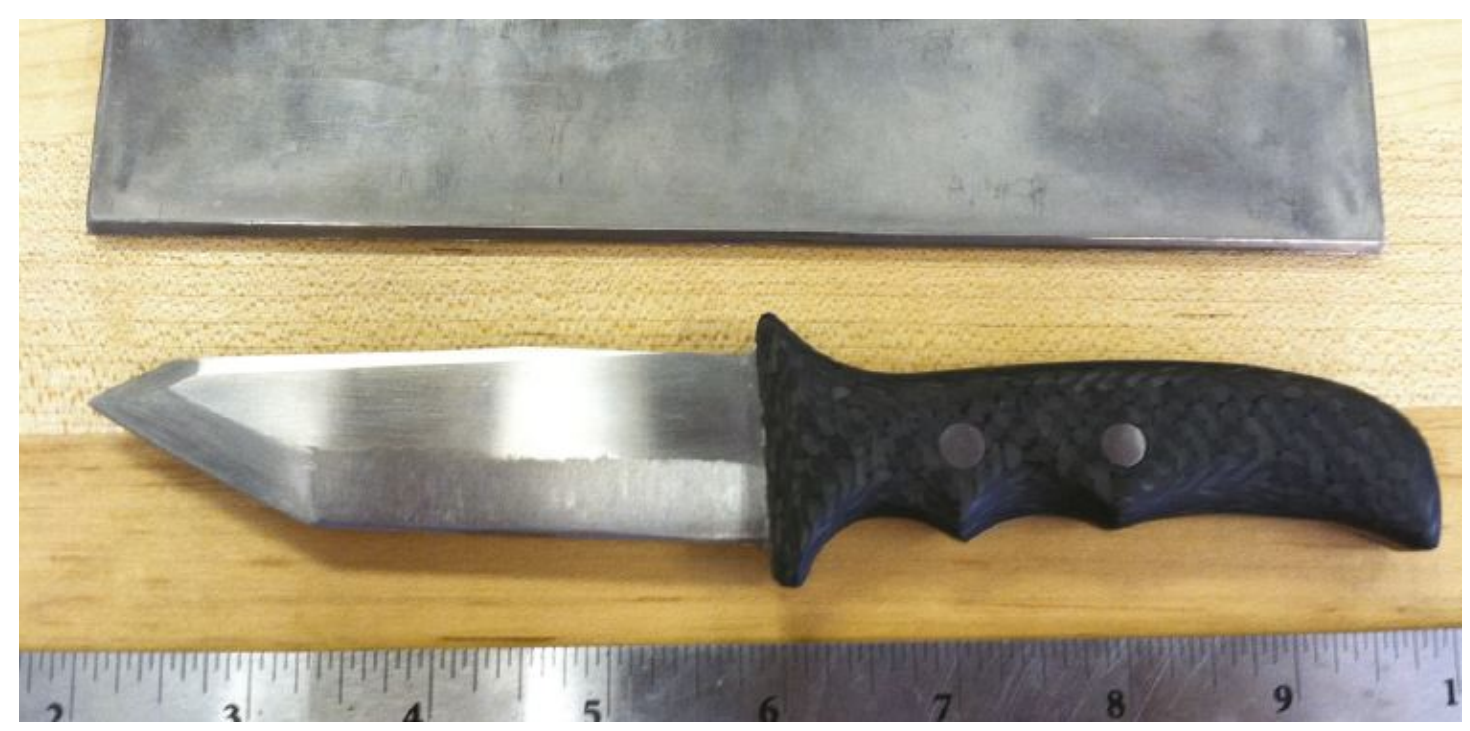


Figure 79 Examples of a variety of net-shaped parts of the Ti-based BMG matrix composite DV1 (TiZrVCuBe) showing how complex shapes can be cast with Ti-based composites. Bolts, washers, hollow tubes, and rods are demonstrated [571].

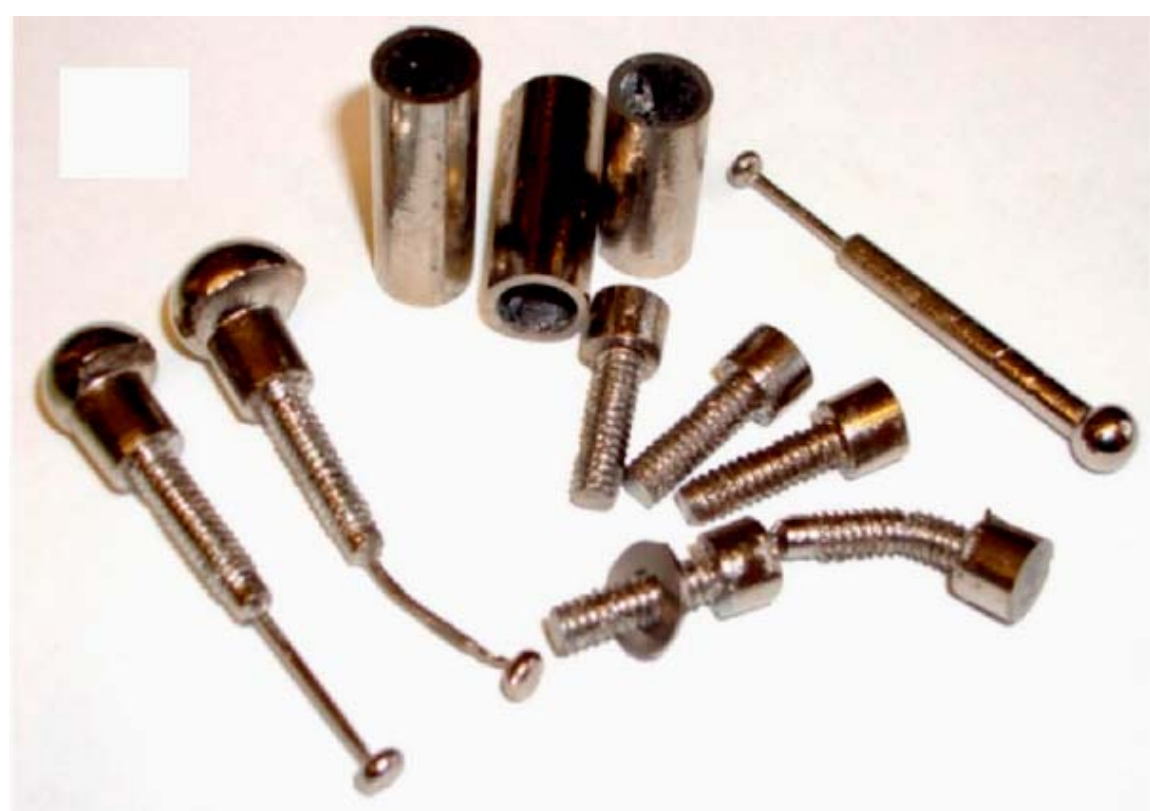


Figure 80 Various processing methods to fabricate MGMCs [572].

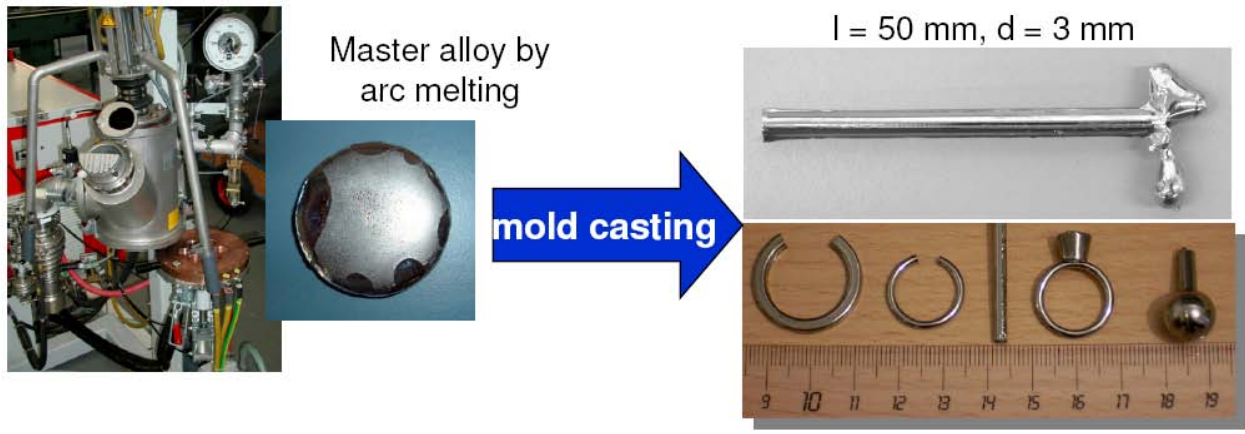

injection

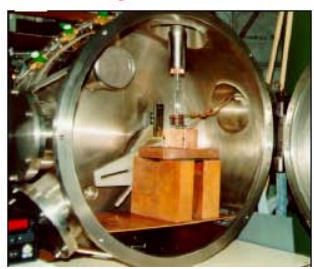

centrifugal

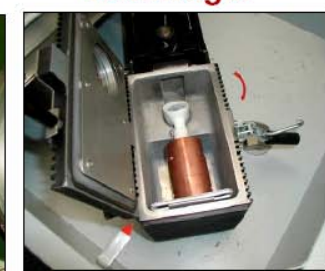

cold crucible
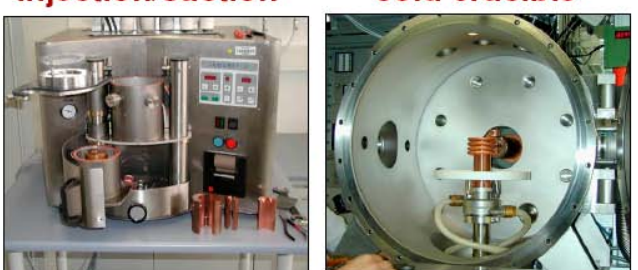